\title{
Critical dynamics of gelling polymer solutions
}

\author{
Dissertation \\ zur Erlangung des Doktorgrades \\ der Mathematisch-Naturwissenschaftlichen Fakultäten \\ der Georg-August-Universtiät zu Göttingen
}

\author{
vorgelegt von \\ Henning Löwe \\ aus Gronau (Leine)
}

Göttingen, 2004 
D7

Referentin: Prof. Dr. A. Zippelius

Korreferent: Prof. Dr. T. Salditt

Tag der mündlichen Prüfung: 09.12.2004 
But in this land of polyurethane, things are apt to get a bit hot. (Les Claypool) 


\section{Contents}

1 The gelation transition 1

1.1 Introduction . . . . . . . . . . . . . . . . . 1

1.2 Connectivity properties near the gel point $\ldots \ldots \ldots \ldots \ldots$

1.2 .1 Classical theory . . . . . . . . . . . . . . . . . . 4

1.2 .2 Bond-percolation . . . . . . . . . . . . . . . 5

1.3 Dynamical properties near the gel point . . . . . . . . . . 8

1.3.1 Scaling assumption of critical rheology . . . . . . . . . 8

1.3.2 Scaling arguments for critical exponents . . . . . . . . . . 9

1.4 Experimental overview . . . . . . . . . . . . . . . . . 11

1.4 .1 Chemical synthesis . . . . . . . . . . . . . . . . . . 11

1.4.2 Important experimental results . . . . . . . . . . . . . . . 13

1.5 Scope of this work . . . . . . . . . . . . . . . . . . . . 14

1.5 .1 Motivation . . . . . . . . . . . . . . . . . 14

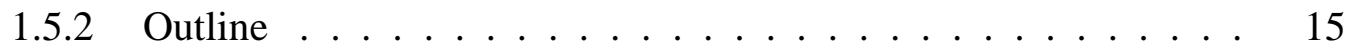

2 Dynamical Model of gelling liquids $\quad 17$

2.1 Static properties of macromolecules . . . . . . . . . . . . 17

2.2 Dynamics of macromolecules in solution . . . . . . . . . . . 18

2.3 Dynamic model of phantom clusters . . . . . . . . . . . . . 23

2.3 .1 Statics of phantom clusters . . . . . . . . . . . . . 23

2.3.2 Rouse model: Free draining approximation . . . . . . . . 26

2.3.3 Zimm model: Preaveraged hydrodynamic interactions . . . . . 26

2.4 Crosslink statistics . . . . . . . . . . . . . . . . . . . . . 28

2.4 .1 Disorder averages . . . . . . . . . . . . . . . . . . . . 28

2.4 .2 Crosslink distributions . . . . . . . . . . . . . . . . . . . . . . 29

2.4 .2 .1 Bond Percolation . . . . . . . . . . . . . . . . 29

2.4.2.2 Erdős-Rényi random graphs . . . . . . . . . . . . . . 29

3 Dynamical signatures of the gelation transition 31

3.1 Stress relaxation . . . . . . . . . . . . . . . . . . . 31

3.1 .1 Stress tensor . . . . . . . . . . . . . . . . . 31

3.1 .2 Linear viscoelasticity . . . . . . . . . . . . . . . . . . . 32

3.1.2.1 Steady shear flow . . . . . . . . . . . . . 33 
3.1.2.2 Oscillatory shear . . . . . . . . . . . . . . . 33

3.1.2.3 Creep flow . . . . . . . . . . . . . . . . . 34

3.1.3 Microscopic expression for stress tensor . . . . . . . . . . . . 34

3.2 Density fluctuations . . . . . . . . . . . . . . . . . . . . . 35

3.2 .1 Dynamic light scattering . . . . . . . . . . . . . . . 35

3.2.2 Coherent and incoherent scattering function . . . . . . . . 37

3.2 .3 Cluster diffusion . . . . . . . . . . . . . . . 38

3.2.4 Nonlinear dynamic susceptibility $\ldots \ldots \ldots \ldots . \ldots . \ldots 38$

4 Solution of the Rouse-Zimm model 41

4.1 Solution of the equation of motion . . . . . . . . . . . . . 41

4.1.1 The simplest case: Rouse model without external flow . . . . . 41

4.1 .2 Rouse-Zimm model with shear flow . . . . . . . . . . . . . . 43

4.1.3 Rouse-Zimm model without external flow . . . . . . . . . . . . . 46

4.1.4 Consistency of the Zimm model . . . . . . . . . . . . . . 47

4.2 Viscoelasticity in the Rouse-Zimm model . . . . . . . . . . . . . 47

4.2 .1 Stress tensor . . . . . . . . . . . . . . . . . . . . 47

4.2 .2 Stress relaxation function . . . . . . . . . . . . . . . . . . . 49

4.2 .3 Shear viscosity . . . . . . . . . . . . . . . . . . . . . 49

4.2 .4 Normal stresses . . . . . . . . . . . . . . . . . . . . . . . . 49

4.2 .5 Recoverable compliance . . . . . . . . . . . . . . . 50

4.3 Density fluctuations within the Rouse-Zimm model . . . . . . . . . 50

4.3 .1 Diffusion constants . . . . . . . . . . . . . . . . 50

4.3.2 Incoherent scattering function . . . . . . . . . . 52

4.3 .3 Dynamic nonlinear susceptibility . . . . . . . . . 52

5 Methods

5.1 Disorder averages . . . . . . . . . . . . . . . . . . 55

5.1 .1 Cluster decomposition of observables . . . . . . . . . . 56

5.1 .2 Critical behavior of observables . . . . . . . . . . . 57

5.2 Random resistor networks . . . . . . . . . . . . . . . . 58

5.2 .1 From Rouse dynamics to random resistor networks . . . . . . 58

5.2.2 How to apply the resistor analogy: Shear viscosity . . . . . . . 61

5.2.3 Scaling properties of random resistor networks . . . . . . . 63

5.2.3.1 Erdős-Rényi random graphs . . . . . . . . . . . . 63

5.2 .3 .2 Bond percolation . . . . . . . . . . . . . 64

5.3 Random walks on fractals . . . . . . . . . . . . . . . 66

5.3.1 Connecting Rouse dynamics to a blind ant's random walk . . . 66

5.3.2 Scaling properties of the blind ant's random walk . . . . . . . 68

5.4 Density of states of the connectivity matrix . . . . . . . . . . . 69

5.4.1 Connecting Rouse dynamics to the density of states of the graph Laplacian 69

5.4.2 Scaling properties of the density of states . . . . . . . . 70

5.5 Numerical methods . . . . . . . . . . . . . . . . . . . . . 71 
5.5 .1 Erdôs-Rényi random graphs . . . . . . . . . . . . . . . . . 72

5.5 .2 Bond percolation . . . . . . . . . . . . . . . . 73

6 Results 79

6.1 Rouse Model . . . . . . . . . . . . . . . . . . . . . . . . . . 79

6.1 .1 Shear viscosity . . . . . . . . . . . . . . . . . . . . 79

6.1 .2 First normal stress coefficient $\ldots \ldots \ldots \ldots$. . . . . . . . 81

6.1 .3 Recoverable compliance . . . . . . . . . . . . . . . . . 82

6.1 .4 Incoherent Scattering function . . . . . . . . . . . . . . 83

6.1 .5 Order parameter susceptibility . . . . . . . . . . . . . . . 87

6.2 Zimm model . . . . . . . . . . . . . . . . . . . . . . . . . . . . 89

6.2.1 Shear viscosity and first normal stress coefficient $\ldots \ldots \ldots$. . . 89

$6.2 .1 .1 \quad$ Numerical results . . . . . . . . . . . . . . . . 89

6.2.1.2 A reference system: Ring polymers . . . . . . . . . . 92

6.2.1.3 Critical behavior . . . . . . . . . . . . . . . . . 94

6.2 .2 Recoverable compliance . . . . . . . . . . . . . . . . . 95

6.2 .3 Cluster diffusion . . . . . . . . . . . . . . . . . . 95

6.2.3.1 Kirkwood diffusion constant . . . . . . . . . . 95

6.2.3.2 Diffusion constants . . . . . . . . . . . . 96

6.2 .4 Incoherent scattering function $\ldots \ldots \ldots \ldots$

6.2 .5 Effective diffusion constant . . . . . . . . . . . . . 100

$\begin{array}{lll}7 \text { Discussion } & 101\end{array}$

7.1 Summary of results . . . . . . . . . . . . . . . . . . 101

7.2 Comparison to experiments and simulations $\ldots \ldots \ldots \ldots \ldots$

7.3 Comparison to scaling theories . . . . . . . . . . . . . . . 108

7.3.1 The electrical analogy . . . . . . . . . . . . . . . 108

7.3.2 The relevance of excluded volume interactions . . . . . . . 109

7.3.3 Viscoelasticity in the absence of hydrodynamic interactions . . . 111

7.3.4 Viscoelasticity in the presence of hydrodynamic interactions . . . 113

7.3 .5 Density fluctuations . . . . . . . . . . . . . . . . 114

7.4 Conclusions . . . . . . . . . . . . . . . . . . . 115

7.5 Open problems . . . . . . . . . . . . . . . . . 116

7.6 Outlook . . . . . . . . . . . . . . . . . . . 119

$\begin{array}{ll}\text { A The equation of motion } & 121\end{array}$

B The connectivity matrices $\quad 123$

B.1 Spectral properties of the graph Laplacian . . . . . . . . . . . . . 123

B.2 Relation between a symmetric or nonsymmetric, dynamic matrix in the Zimm model 124

B.3 Consistency condition for the Zimm model . . . . . . . . . . . 125

B.4 Proof of the resistance formula $(5.16) \ldots \ldots \ldots \ldots \ldots$ 
C Correlation functions $\quad 127$

C.1 Long time behaviour $\ldots \ldots \ldots \ldots \ldots \ldots \ldots$

D Equilibrium averages $\quad 129$

D.1 Pair distributions . . . . . . . . . . . . . . . . . . . . . . . . . . . 129

D.2 Preaveraging of the mobility matrix $\ldots \ldots \ldots \ldots \ldots$

E Asymptotic evaluations of integrals and sums $\quad 133$

E.1 Critical behaviour of disorder averages . . . . . . . . . . . . . . 133

E.2 Long-time decay of the incoherent scattering function . . . . . . . . . 134

E.3 Moments of the resistance distribution . . . . . . . . . . . . . 135

\begin{tabular}{lll}
\hline The Jensen inequality & 137
\end{tabular}

G Disorder averages from the Leath-algorithm 139

H Ring polymers in the Zimm model 141

I A variational bound to the viscosity 145

$\begin{array}{ll}\text { References } & 147\end{array}$

$\begin{array}{ll}\text { Acknowledgement } & 161\end{array}$

$\begin{array}{ll}\text { Curriculum vitae } & 162\end{array}$

$\begin{array}{ll}\text { List of publications } & 163\end{array}$ 


\section{Chapter 1}

\section{The gelation transition}

\subsection{Introduction}

Charles Goodyear's discovery of the vulcanization of rubber in 1839 has probably given the initial impetus to what has become an independent research area of physics in the meantime, namely soft matter physics. Goodyear observed that adding a sufficient amount of sulfur to a liquid cis-polyisoprene melt yields a peculiar, "soft", solid-state material: rubber. In more contemporary terms, Goodyear discovered a special example of system which exhibit a sol-gel or gelation transition: If a liquid macromolecular system is subjected to a chemical reaction mechanism, crosslinking, which gradually and randomly joins the constituents to form larger and larger macromolecules, the system undergoes a transition from a complex fluid (sol-phase) to a solid state (gel-phase) (see Fig. 1.1). If the crosslinking imposes a permanent constraint on the degrees of freedom the gelation process is referred to as chemical or strong gelation. Permanent crosslinks are formed by chemical bonds which are energetically stable when subjected to thermal fluctuations. For chemical crosslinks the gelation transition is an equilibrium, second-order thermodynamic phase transition from a complex liquid to an amorphous solid state. The transition is controlled by a parameter, the number density of crosslinks $c$. In contrast, in physical or weak gels the energy of the bonds is of order of the thermal energy $k_{\mathrm{B}} T$. Thermal fluctuations cause breaking and reformation of the crosslinks and prevent the system from exhibiting a true phase transition. In the following, exclusively chemical gels are considered.

If a gelling system is sufficiently close to its critical point $c_{\text {crit }}$ the upcoming transition is signalled by striking static and dynamical critical phenomena. One of the most fundamental, dynamic signatures in the liquid phase is the divergence of the shear viscosity $\eta \sim\left(c_{\text {crit }}-c\right)^{-k}$ when the critical density is approached from below. It signals the presence of increasing solid-like regions in the fluid and may be regarded as the precursor of another signature, which is actually static in nature, the emergence of shear rigidity in the gel phase. A nonzero shear modulus $G \sim\left(c_{\text {crit }}-c\right)^{\mu}$ emerges continuously in the gel phase when the critical crosslink density is increased above its critical value. The situation is schematically depicted in Fig. 1.2. The soft and sloppy nature of the rubbery phase is 

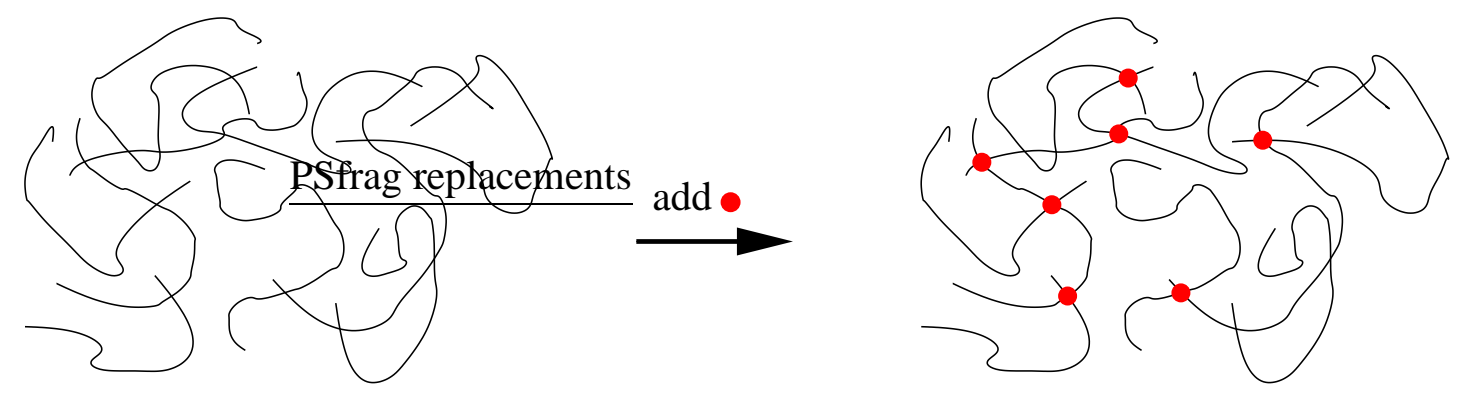

Figure 1.1: Gelation transition from a complex, macromolecular fluid (sol phase) to an amorphous solid (gel phase) by random crosslinking (indicated by red circles)

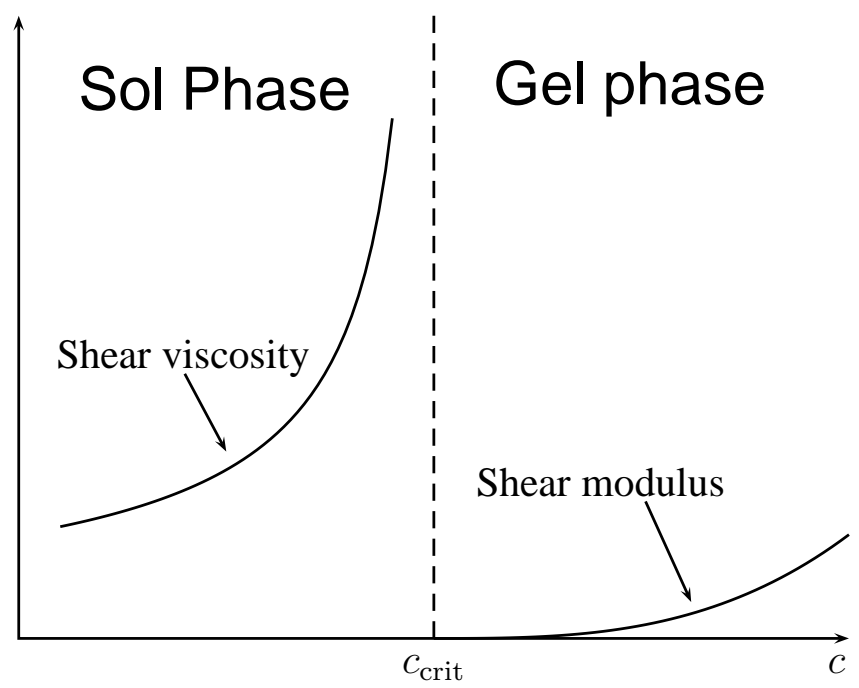

Figure 1.2: Schematic depiction of the shear viscosity and the shear modulus as functions of the crosslink density $c$ in the vicinity of gelation transition.

due to the entropic elasticity of the macromolecular building blocks.

This solidification transition bears some fundamental differences to a first order liquidsolid phase transition of simple fluids and is, to some extent, reminiscent of the glass transition. In the sol phase as well as in supercooled liquids correlation functions no longer decay exponentially in time. Instead, a critical slowing down is observed which is reflected by a stretched exponential (Kohlrausch-William-Watts) decay of time dependent correlation functions such as the incoherent scattering function $S(\boldsymbol{q}, t) \sim \exp \left(-\left(t / t_{q}\right)^{\alpha}\right)$, with $\alpha<1$. Such a slowing down is commonly related to the presence of a broad distribution of relaxation times which reflects distinctly relaxing spatial domains. However, the issue of identifying these domains allows for a clear-cut distinction between the gelation transition and the glass transition. Within gelation the identification of thermal and quenched degrees of freedom is unambiguous: Monomers equilibrate in the presence of crosslinks which constitute well defined quenched degrees of freedom. Slowly relaxing domains are then easily identified as large clusters. In contrast, when lowering the temperature in 
structural glasses the identification of quenched degrees of freedom is neither simple nor unique. Moreover, it is still being questioned if there is an underlying equilibrium phase transition at all.

A further remarkable feature of a gelling system is the absence of critical opalescence at the critical point. This is contrast to to e.g. the first order liquid-vapor transition of a simple fluid or to the second order phase-separation transition of a binary fluid. The latter examples do exhibit critical, macroscopic density fluctuations and hence large fluctuations in the refractive index of the material which is easily revealed by light scattering experiments. Macroscopic concentration fluctuations are associated with a diverging spatial correlation length. Gelling systems do not exhibit critical opalescence and hence, fluctuations of the monomer density must remain short ranged throughout the transition. It is rather the divergence of a more subtle correlation length which gives rise to long-ranged correlations, the connectivity correlation length. Therefore, the characterization of connectivity properties amounts to one of the fundamental tasks for a theoretical description of gelling systems.

\subsection{Connectivity properties near the gel point}

For a characterization of connectivity properties of a gelling system, the gelling system may be regarded as a population of macromolecules which consist of identical building blocks, the monomers. The system may be characterized by its mass distribution $\tau_{n}$ which is the number of clusters (per monomer) which consist of $n$ monomers. It is customary to use the term size for the number of monomers in a cluster and $\tau_{n}$ is commonly referred to as the cluster size distribution. The quantity $n \tau_{n}$ is the fraction of monomers which belong to clusters of size $n$.

The cluster size distribution of a gelling system depends on the process of cluster formation in the reaction bath which is a complicated, dynamic process. Basically, there exist two main theoretical frameworks which aim at the characterization of the cluster size distribution for gelation, kinetic gelation and equilibrium gelation (see [MaAd91] or Ch. 6 in [RuCo03]).

The main purpose of kinetic gelation is the prediction of the evolution of cluster growth. It emphasizes the fact that crosslinking is not an instantaneous process. A heuristic Smoluchowski equation for the cluster sizes $\tau_{n}$ is proposed. This equation is governed by reaction kernels $K_{n, m}$ which describe the rates of cluster-cluster aggregation processes, i.e. the formation of an $(n+m)$-cluster from the reaction of an $n$-cluster with an $m$-cluster. By ad-hoc assumptions for the functional form of $K_{n, m}$ this approach allows predicting generic features of gelation such as the cluster size distribution $\tau_{n}$, a gel point, which is a point $t_{\text {gel }}$ in time, and a diverging average cluster size $n_{\mathrm{w}}$. Moreover, the kinetic approach can predict time regimes with characteristic features of diffusion-limited cluster growth and time regimes in which cluster growth is reaction-limited. However, kinetic gelation is not able to predict the internal structure of the clusters, i.e. the nature of connectedness.

In contrast, the equilibrium gelation approach is motivated by the idea that the gelation 
transition is the signature of an underlying percolation transition. Equilibrium gelation completely ignores kinetic aspects of cluster growth and might be interpreted as if crosslinks were instantaneously established between neighboring macromolecules. A (bond) percolation transition [StAh95, BuHa96] is a connectivity transition between points, called vertices or sites with fixed positions in space. The points are to be connected by edges or bonds with certain probability $p$. This process gives rise to a population of cluster sizes. The connection to gelation is then established by identifying the bonds with crosslinks and the vertices with monomers.

\subsubsection{Classical theory}

The simplest percolation type approach to gelation constitutes the so-called classical theory of gelation which was initiated by Flory [Flo41a, Flo41b, Flo41c] and Stockmayer [Sto43, Sto44] and is at heart a tree approximation of connectivity. The classical theory assumes that a system of identical $f$-functional units are to be crosslinked, randomly and covalently, within the following three assumptions: First, only those aggregations are allowed which lead to treelike structures. Second, all unreacted monomers have the same reactivity. Third, the reaction probability is simply proportional to the mean concentration of reactant. As a consequence of these assumptions, the model amounts to a mean field approach of gelation and is equivalent to percolation on a Bethe-lattice or Cayley-tree (see Fig. 1.3). This simple percolation problem can be solved exactly and thereby reveals essential features of the percolation transition. The cluster size distribution

$$
\tau_{n} \sim n^{-5 / 2} \exp \left\{-n / n^{*}\right\}
$$

obeys a scaling form in the scaling variable $n / n^{*}$. It decays algebraically for cluster sizes smaller than the cutoff cluster size $n^{*}$, which is effectively the largest cluster size in the system since clusters with size $n>n^{*}$ are exponentially rare. The largest cluster size diverges with vanishing distance $\varepsilon:=\left|p_{c}-p\right| / p_{c}$ to the critical point as $n^{*} \sim \varepsilon^{-2}$. For the diluted Bethe lattice with functionality $\varphi$ the critical point is simply given by $p_{\text {crit }}=1 /(\varphi-$ 1). Above the critical point the Flory-Stockmayer theory predicts that a finite fraction of monomers belongs to an infinite cluster, the gel-fraction or the macroscopic cluster. It is the order parameter of percolation and for the Bethe-lattice it vanishes continuously,

$$
S_{\infty} \sim \varepsilon^{1}
$$

However, the predictions of the Flory-Stockmayer theory for the exponents in (1.1) and (1.2) turned out to apply only for very special conditions: the crosslinking of long polymer chains in dense melts. This was first understood by de Gennes [Gen77]. Long polymer chains in the dense state highly overlap and a particular monomer is surrounded by a very high (mean field like) number of other monomers. Only within a very narrow, critical region whose width is inversely proportional to the length of the chains where the Flory-Stockmayer fails. Likewise it fails for systems with smaller building blocks in any case. Against this background Stauffer and de Gennes [Gen75, Sta76, StCo82] proposed 

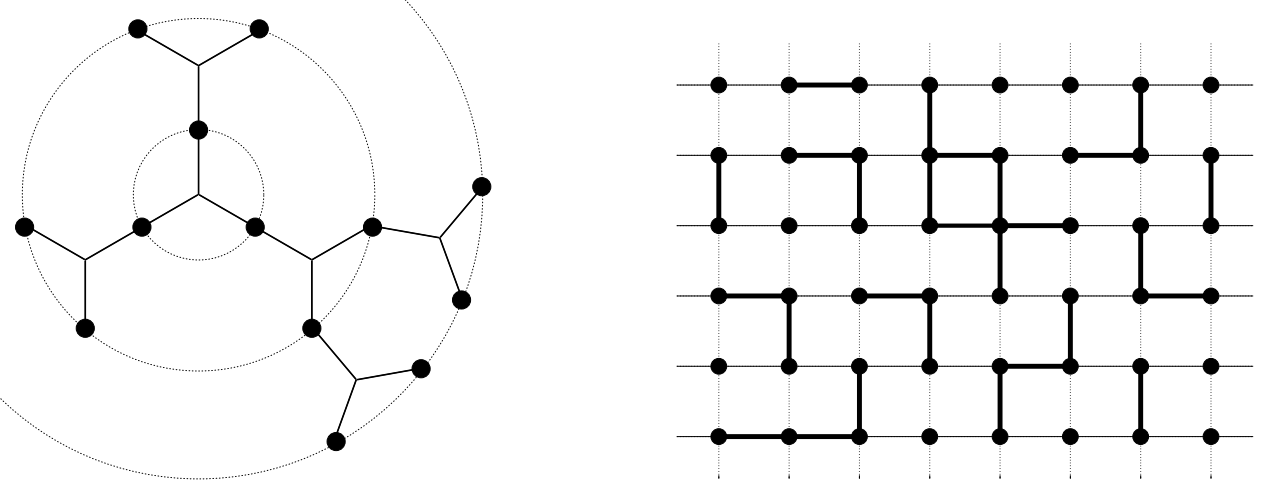

Figure 1.3: Examples for percolation. Gelation of three-functional monomers (left), reminiscent of percolation on the Bethe lattice and percolation on the square lattice (right).

percolation on a three dimensional hypercubic lattice as a model for gelation. This percolation process implicitly takes correlations between crosslinks into account, since the number of possible crosslinks emanating from one monomer is bounded according to the coordination number of a point in the regular lattice. This should somehow reflect the bounded number of neighboring monomers in a typical, irregular configuration of a three dimensional gelling fluid. In fact, the Flory-Stockmayer theory, i.e. percolation on a Bethelattice turns out to be the limit of infinite spatial dimensionality of lattice percolation and it has the same critical exponents as lattice bond percolation at and above its upper critical dimension $d=6$.

\subsubsection{Bond-percolation}

Bond percolation is the connectivity transition on the hypercubic lattice $\mathbb{Z}^{d}$ if neighboring lattice points are independently connected by a bond with probability $p$ and remain disconnected with probability $1-p$. Similar to other phase transitions it is convenient to allow for a varying spatial dimension $d$, even if experiments are restricted to $d=3$. In the following, a detailed description of bond percolation is given since it is important for later purposes. Introductions to percolation theory and its applications can be found, e.g. , in [StAh95, Sah94, BuHa96].

It is a well-established fact of the scaling description of bond percolation [StAh95] that the cluster size distribution of finite clusters obeys a scaling law

$$
\tau_{n}=n^{-\tau} f\left(n / n^{*}\right)
$$

in the vicinity of the of the critical probability $c_{\text {crit }}$ which is non-universal and depends on the dimensionality $d$ and details of the lattice. The scaling function $f(x)$ decays exponentially for large arguments [Gri89, Ch. 5.3] and approaches a nonzero constant for small arguments. The cutoff cluster size $n^{*} \sim \varepsilon^{-1 / \sigma}$ diverges with a critical exponent $\sigma$. By 


\begin{tabular}{l|l} 
Connectivity properties & Spatial arrangement \\
\hline \hline$\beta=(\tau-2) / \sigma$ & $\nu=\beta /\left(d-d_{\mathrm{f}}^{(\mathrm{H})}\right)$ \\
$\gamma=(3-\tau) / \sigma$ & $\nu=1 /\left(\sigma d_{\mathrm{f}}^{(\mathrm{H})}\right)$ \\
& $\beta=\frac{\nu}{2}(d-2+\tilde{\eta})$
\end{tabular}

Table 1.1: Important scaling relations of percolation. As a consequence, there are only two independent exponents among $\tau, \sigma, \beta, \gamma, \nu, d_{\mathrm{f}}^{(\mathrm{H})}, \tilde{\eta}$.

means of the cluster size distribution one can compute the weight-averaged cluster size. It diverges at the transition

$$
n_{\mathrm{w}}=\sum_{n=1}^{\infty} n^{2} \tau_{n} \sim \varepsilon^{-\gamma}
$$

with a critical exponent $\gamma$. This can be easily understood by replacing the sum in (1.4) by an integral which enables one to express $\gamma$ in terms of the exponents $\sigma$ and $\tau$ by the scaling relation $\gamma=(3-\tau) / \sigma$. Note that the weight-averaged cluster size $n_{\mathrm{w}}$ diverges with a different exponent as the cutoff cluster-size $n^{*}$, the latter often being referred to as the $z$-averaged cluster size in polymer physics. It is given by $n^{*}=\sum_{i=1}^{\infty} n^{3} \tau_{n} / \sum_{i=1}^{\infty} n^{2} \tau_{n}$.

The order parameter of percolation, the gel fraction $S_{\infty}$ is the fraction of vertices which belong to the infinite cluster. It vanishes continuously when the transition is approached from above,

$$
S_{\infty} \sim \varepsilon^{\beta}
$$

Since each vertex belongs either to a finite cluster or to the infinite cluster one has $\sum_{n=1}^{\infty} n \tau_{n}+S_{\infty}=1$, where the summation extends over all arbitrarily large, but finite clusters. This equality implies the normalization $\sum_{n=1}^{\infty} n \tau_{n}=1$ of the cluster size distribution below the threshold $c<c_{\text {crit }}$ and the scaling relation $\beta=(\tau-2) / \sigma$ between the critical exponents.

Up to this point only the connectivity properties of the percolation ensemble have been characterized. The quantities (1.3), (1.5), (1.4) and the corresponding exponents $\beta, \gamma, \tau, \sigma$ do not refer to the spatial arrangement of the points. The associated scaling relations among the critical exponents which solely describe the connectivity are summarized in Tab. 1.1.

It is the fact that the positions of all points are rigidly fixed on the lattice $\mathbb{Z}^{d}$ which allows establishing relations between the connectivity properties and the spatial arrangement of the points. The connectedness correlation function $g(r)$ is proportional to the probability that two vertices which belong to the same cluster are separated by the Euclidean distance $r$. Its scaling form

$$
g(r) \sim r^{-(d-2+\tilde{\eta})} \mathrm{e}^{-r / \xi}
$$

is governed by a single, characteristic length scale, the correlation length $\xi$. This is the connectivity counterpart of the concentration fluctuation length in thermal phase transitions mentioned in the introduction. If the transition is approached, connectivity correlations 


\begin{tabular}{l|l|l|l|l|l|l|} 
& $\tau$ & $\sigma$ & $\beta$ & $\gamma$ & $d_{\mathrm{f}}^{(\mathrm{H})}$ & $\nu$ \\
\hline \hline$d=3$ & 2.18 & 0.45 & 0.41 & 1.80 & 2.53 & 0.88 \\
\hline$d=6$ & $5 / 2$ & $1 / 2$ & 1 & 1 & 4 & $1 / 2$
\end{tabular}

Table 1.2: Numerical values of percolation exponents in spatial dimension $d=3$ and in the mean fi eld limit $d=6$. (taken from [StAh95])

become increasingly long-ranged which is indicated by the divergence

$$
\xi^{2}:=\frac{\sum_{r \in \mathbb{Z}^{d}} r^{2} g(r)}{\sum_{r \in \mathbb{Z}^{d}} g(r)} \sim \varepsilon^{-2 \nu}
$$

The divergence of the correlation length has important consequences on the homogeneity of the ensemble. At the critical point the system is a self-similar "fractal" collection of clusters which lacks any characteristic length scale. The radius of gyration $R_{n}^{2}$ of clusters with $n$ sites follows a power law which defines the Hausdorff-fractal dimension $d_{\mathrm{f}}^{(\mathrm{H})}$,

$$
R_{n}^{2} \sim n^{2 / d_{\mathrm{f}}^{(\mathrm{H})}}
$$

Likewise, the Hausdorff fractal dimension characterizes the mass $n(r)$ of the infinite cluster in a sphere of radius $r$ via $n(r) \sim r_{\mathrm{f}}^{d_{\mathrm{f}}^{(\mathrm{H})}}$. Slightly above the critical point the system can be regarded as self similar only on spatial length scales $r<\xi$. For larger $r$ the infinite cluster is homogeneous and the size-mass relation takes the usual form $n(r) \sim r^{d}$. Hence, the probability that an arbitrary vertex within a sphere of radius $r<\xi$ above the critical point belongs to the infinite cluster is given by the ratio $S_{\infty}=r_{\mathrm{f}}^{d_{\mathrm{f}}^{(\mathrm{H})}} / r^{d}$, that is, the mass fraction of the infinite cluster in the sphere divided by the total mass of the sphere. The limiting case $r=\xi$ yields the scaling relation $\beta=\nu\left(d-d_{\mathrm{f}}^{(\mathrm{H})}\right)$. This relation is an example for a hyperscaling relation since it involves the spatial dimension $d$ explicitly.

The correlation length $\xi$ is can be regarded as the radius of the largest cluster of size $n^{*}$ and therefore, by (1.8), one gets another, useful scaling relation $1 / \sigma=\nu d_{\mathrm{f}}^{(\mathrm{H})}$.

As a consequence of all scaling relations there remain only two independent exponents which characterize the critical properties of lattice bond percolation completely. The scaling relation are summarized in Tab. 1.1. The exponents depend on the dimensionality $d$. Bond percolation has an upper critical dimension $d_{\mathrm{uc}}=6$ which means that for $d \geq 6$ the exponents are given by their mean field values. Precisely at $d=6$ the mean field scaling is influenced by logarithmic corrections. Numerical values for the percolation exponents in $d=3$ and in the mean field limit $d=6$ are summarized in Tab. 1.2. 


\subsection{Dynamical properties near the gel point}

\subsubsection{Scaling assumption of critical rheology}

Beside characterizing the connectivity properties of the gelling systems by the percolation picture, the main goal is indeed the prediction of critical behaviour of static and dynamical physical quantities. The most prominent examples of physical quantities are the aforementioned shear viscosity $\eta \sim \varepsilon^{-k}$ and the shear modulus $G \sim \varepsilon^{\mu}$. Both quantities are rheological quantities.

The rheological behaviour of a material is quite generally described by the frequency dependent, complex shear modulus $\widehat{G}(\omega)=\widehat{G}^{\prime}(\omega)+\mathrm{i} \widehat{G}^{\prime \prime}(\omega)$ (see Ch. 1 [Fer61]) where $\mathrm{i}=\sqrt{-1}$ denotes the imaginary unit. The real part $\widehat{G}^{\prime}(\omega)$ is the storage modulus which measures the energy storage per cycle in an oscillatory shear excitation at frequency $\omega$. The imaginary part $\widehat{G}^{\prime \prime}(\omega)$ is the loss modulus which measures the energy dissipation per cycle. The complex modulus is defined, such that the viscosity is recoverd by the low frequency behaviour according to $\eta:=\lim _{\omega \rightarrow 0} \widehat{G}(\omega) /(\mathrm{i} \omega)=\lim _{\omega \rightarrow 0} \widehat{G}^{\prime \prime}(\omega) / \omega$ and likewise the shear modulus $G:=\lim _{\omega \rightarrow 0} \widehat{G}(\omega)=\lim _{\omega \rightarrow 0} \widehat{G}^{\prime}(\omega)$.

In the vicinity of the transition it is reasonable to assume that the complex modulus $\widehat{G}$ obeys a scaling form [DuDe87, GoGo92]

$$
\widehat{G}(\omega) \sim(\mathrm{i} \omega)^{\Delta} \mathcal{F}_{\widehat{G}}^{( \pm)}\left(\mathrm{i} \omega / \omega^{*}\right)
$$

with a characteristic frequency $\omega^{*}$ which is the inverse characteristic time scale $t^{*}$ of the gelling system. The critical slowing down suggest a divergence of the time scale

$$
t^{*} \sim 1 / \omega^{*} \sim t_{\mathrm{mic}} \varepsilon^{-z},
$$

with a critical exponent $z$ which is referred to as the dynamic exponent and a microscopic time scale $t_{\text {mic }}$. The scaling functions $\mathcal{F}_{\widehat{G}}^{( \pm)}$are allowed to differ in the fluid phase (-) and in the gel phase $(+)$.

In order to obtain a well defined viscosity for $\omega \downarrow 0$ the scaling function must have the functional form $\mathcal{F}_{\widehat{G}}^{(-)}(w) \sim w^{1-\Delta}$ for $w \downarrow 0$ in order to cancel out the prefactor. This implies a divergence $\eta \sim\left(t^{*}\right)^{1-\Delta}$ for the viscosity and hence the viscosity exponent $k$ can be related to the exponents of the complex shear modulus via $k=z(1-\Delta)$. Likewise, a finite shear modulus above the critical point requires $\mathcal{F}_{\widehat{G}}^{(+)}(w) \sim w^{-\Delta}$ which implies $G \sim\left(t^{*}\right)^{\Delta}$ and the scaling relation $\mu=z \Delta$.

At finite, intermediate frequencies $\omega^{*} \ll \omega \ll t_{\text {mic }}^{-1}$ the complex modulus is expected to be finite for $\varepsilon \downarrow 0$. Therefore, both scaling functions must approach a constant for $|w| \rightarrow \infty$ and the complex modulus obeys a power law

$$
\widehat{G}(\omega) \sim(\mathrm{i} \omega)^{\Delta},
$$

which is also the behavior at the critical point for all frequencies $\omega \ll t_{\text {mic }}^{-1}$. 
In summary, the scaling assumption (1.9) for the complex modulus gives rise to the fundamental scaling relations between the exponents of critical rheology

$$
z=k+\mu \quad \Delta=\frac{\mu}{k+\mu}
$$

i.e. critical viscoelastic behavior is also described by two independent exponents. However, this scaling approach only provides relations among the exponents rather than estimating their numerical values. For that purpose further assumptions are required.

\subsubsection{Scaling arguments for critical exponents}

A suitable starting point for estimating critical exponents of viscoelasticity is to examine typical time scales of the gelling system. In the absence of kinetic aspects of cluster formation, the most fundamental time scale associated with a typical percolation cluster of size $n$ is the diffusive time $t_{n}$ it takes to diffuse a distance of its own radius $R_{n}$, viz

$$
t_{n}=\frac{R_{n}^{2}}{D_{n}}
$$

The cluster's diffusion constant is denoted by $D_{n}$. Since the cluster is a fractal object its radius $R_{n}$ can be related to a fractal dimension $R_{n} \sim n^{1 / d_{\mathrm{f}}}$. This fractal dimension $d_{\mathrm{f}}$ of a cluster is not necessarily given by the Hausdorff fractal dimension $d_{\mathrm{f}}^{(\mathrm{H})}(1.8)$ of percolation clusters on the lattice. This is due to the fact that the spatial configuration of the cluster in the fluid depends on all interactions with the surrounding medium. For instance, it is well known that the presence of a good solvent causes swelling of the clusters [DoEd88, Ch. 2.5] and hence a decrease of the fractal dimension $d_{\mathrm{f}}<d_{\mathrm{f}}^{(\mathrm{H})}$. The fundamental problem of estimating time scales in (1.13) has now been shifted to the problem of estimating diffusion constants.

If solvent effects are believed to be relevant for the dynamics results from the Zimm model for linear polymer chains are generalized to percolation clusters. The Zimm model describes dynamics of polymers with dominant hydrodynamic interactions. The characteristic feature of the Zimm model is the prediction $D_{n} \sim 1 / R_{n}$ for the diffusion constant [DoEd88]. This dependence on the radius is easily understood by the following simple scaling argument which uses the well known Einstein relation for the diffusion constant of a Brownian particle in terms of its friction constant: The diffusion constant $D_{n}$ is then taken to be proportional to the inverse friction coefficient $\zeta_{n}$ of that cluster, $D_{n} \sim k_{\mathrm{B}} T / \zeta_{n}$. By regarding the cluster as a spherical object the friction constant is estimated by Stokes' law of friction, $\zeta_{n} \sim 6 \pi \eta_{\mathrm{s}} R_{n}$, where $\eta_{\mathrm{s}}$ denotes the viscosity of the solvent. The combination of these arguments yields the so called Stokes-Einstein relation for the diffusion constant of a cluster of size $n$,

$$
D_{n} \sim R_{n}^{-1}
$$

If the Stokes friction is generalized to $d$ spatial dimensions via $\zeta_{n} \sim R_{n}^{d-2}$ [MaAd89], the scaling of the typical relaxation time of a cluster of size $n$ with dominant hydrodynamic 
interactions becomes

$$
t_{n} \sim R_{n}^{d} \sim n^{d / d_{\mathrm{f}}} \quad(\text { Zimm }) .
$$

The longest time scale $t^{*}$ of the gelling system is now related to the time scale $t_{n^{*}}$ of the largest cluster with size $n^{*} \sim \varepsilon^{-1 / \sigma}$. This implies $t^{*}:=t_{n^{*}} \sim \varepsilon^{-d /\left(\sigma d_{\mathrm{f}}\right)}$ which gives rise to the prediction $z=d /\left(\sigma d_{\mathrm{f}}\right)$ of the dynamic exponent with dominant hydrodynamic interactions [MaAd89].

In dense melts percolation is believed to be applicable directly as far as geometrical properties are concerned. In the absence of solvent the swelling of the clusters is suppressed and the fractal dimension of percolation clusters in dense melts is given by the Hausdorff fractal dimension $d_{\mathrm{f}}^{(\mathrm{H})}$. The dynamics in dense melts in the absence of hydrodynamic interactions is assumed to be described by the Rouse model since the Rouse model has the characteristic feature that all monomers experience the same frictional force. Thus the friction coefficient $\zeta_{n}$ of a cluster of size $n$ is simply proportional to the number of monomers, $D_{n} \sim n^{-1}$. This simple behavior of the diffusion constant can be accommodated with the Stokes-Einstein formula by introducing a size dependent viscosity $\eta_{s}(n)$ [Gen79b, MaAd89] which is assumed to describe the screening of hydrodynamic interactions. The typical relaxation time $t_{n}$ of a Rouse cluster of size $n$ is then given by

$$
t_{n} \sim n R_{n}^{2} \sim n^{2 / d_{\mathrm{f}}^{(\mathrm{H})}+1} \quad \text { (Rouse) }
$$

and likewise the characteristic, divergent relaxation time scale by $t^{*}=t_{n^{*}} \sim \varepsilon^{-(2 \nu+1 / \sigma)}$, where the percolation scaling relation $\nu=1 /\left(\sigma d_{\mathrm{f}}^{(\mathrm{H})}\right)$ has been employed. In fact, the majority of theoretical approaches to gelation are based on the assumptions $(1.15,1.16)$ for the relaxation times.

The viscosity can be estimated from the relaxation times by dimensional analysis. The contribution of the excess viscosity of a cluster of size $n$ is proportional to the relaxation time $t_{n}$. More precisely, $\eta_{n} \sim t_{n} / n$ is the energy which is dissipated by one monomer of a typical cluster of size $n$ in the entire volume during the time $t_{n}$. This implies

$$
\eta_{n} \sim n^{d / d_{\mathrm{f}}-1}
$$

for dominant hydrodynamic interactions in dilute solutions. A scaling relation like (1.17) which relates the viscosity of clusters to its size $n$ is referred to as Mark-Houwink relation (cf. Sec. 1.7.3 in [RuCo03]). The form (1.17) has been proposed by several authors [StCo82, Cat85, Mut85]. By weighting these contributions with the cluster size distribution $\eta=\sum_{n=1}^{\infty} \tau_{n} t_{n}$ one gets the prediction $k=\left(1-\tau+d / d_{\mathrm{f}}\right) / \sigma$ for the viscosity exponent in dilute solutions. The other rheological exponents $\Delta$ and $\mu$ can be obtained by $k$ and $z=d /\left(\sigma d_{\mathrm{f}}\right)$ from the scaling relations (1.12).

A similar calculation for the viscosity from the Rouse relaxation time leads to a critical exponent $k=2 \nu-\beta$. This exponent has been proposed originally by de Gennes [Gen78]. Likewise, this exponent can be obtained by a different argument from dimensional analysis. It states that the viscosity is the product of the longest relaxation time $t^{*}$ and the modulus $G$ on that time scale [CoGi93]. The exponent $\mu$ for the shear modulus is easily obtained by the 


\begin{tabular}{l|l|l|l|l} 
& $k$ & $\mu$ & $\Delta$ & $z$ \\
\hline \hline Rouse & 1.32 & 2.70 & 0.66 & 4.0 \\
\hline electrical Analogy & 0.75 & 1.90 & 0.72 & 2.65
\end{tabular}

Table 1.3: Numerical values of the exponents of critical rheology. Comparison of the so called Rouse exponents with the exponents obtained by the electrical analogy.

following argument. In the absence of a solvent, energy is solely transported via elastically active bonds of the cluster. Since the modulus is an energy density, equipartition predicts $G \sim k_{\mathrm{B}} T / \xi^{d}$ per correlation volume. Percolation theory (see Eq. (1.7)) yields $\xi^{d} \sim \varepsilon^{-\nu d}$ and thus $G \sim \varepsilon^{\mu}$ with $\mu=d \nu$. The remaining rheological exponents are obtained from the scaling relations.

There is another, famous argument for the critical, rheological exponents which is worth mentioning, the so-called electrical analogy proposed by de Gennes. The argument is based on an analogy of the mechanical problem to an electrical one. The viscosity is suggested to diverge as the conductivity $\Sigma$ in a conductor/superconductor transition [Gen78]: If between neighboring vertices of a lattice superconducting bonds are introduced with probability $p$ and normal conducting bonds with probability $1-p$ then the material is a normal conductor for $p<c_{\text {crit }}$ and the conductivity diverges with an exponent $\Sigma \sim\left(c_{\text {crit }}-p\right)^{-s}$. The proposal is then $k=s$ for the viscosity exponent. The exponent for the shear modulus is obtained from an insulator/conductor transition [Gen76a]: If neighboring vertices are connected by a normal conductor with probability $p$ and by an insulator with probability $1-p$ then the material is a conductor for $p>c_{\text {crit }}$ and the conductivity vanishes according to $\tilde{\Sigma} \sim\left(p-c_{\text {crit }}\right)^{\tilde{s}}$. The proposal is then $\mu=\tilde{s}$ for the shear modulus exponent.

In the absence of hydrodynamic interactions, the so called Rouse predictions and the predictions from the electrical analogy are almost always used as competing theoretical predictions for the dynamics in melts. Due to their rather distinct numerical values (see 1.3) experimental findings are either assigned to the first or the latter.

\subsection{Experimental overview}

\subsubsection{Chemical synthesis}

There are different mechanisms of synthesizing chemically crosslinked macromolecular systems. The common feature of these polymerization-processes is the reaction of identical polyfunctional building blocks, the monomers, to dimers, trimers, oligomers, and enventually polymers. If macromolecules are polymerized from distinct types of monomers, the term co-polymerization is preferred. A detailed introduction to the classification of polymerization processes can be found in Flory's textbook [Flo92], Ch. II.

The simplest polymerization process is the reaction of two-functional monomers to 


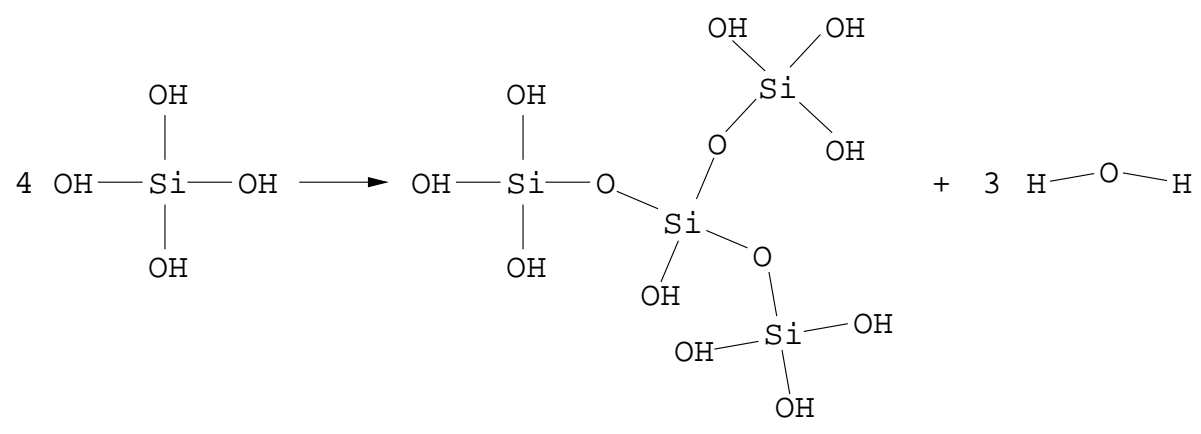

Figure 1.4: Example of a condensation reaction.

linear polymers. Typical examples are polyethylene, polystyrene, polyvenylchlorid (PVC), PMMA, polyurethane. The number of repeating units is referred to as the the degree of polymerization.

However, for gelation it is important that the monomers have functionality greater than two such that polymerization processes lead to branched or nonlinear polymers and eventually to three dimensional space filling networks. A typical example are silica gels, see Fig. 1.4.

A commonly adopted classification scheme subdivides the polymerization processes into two main categories, addition polymerization and condensation polymerization.

Simply speaking, addition polymerization are those processes where the molecular formula for the repeating unit is identical to that of the monomer from which it is derived. An example is provided by polyethylene. The monomer $\mathrm{CH}_{2}=\mathrm{CH}_{2}$ is activated and then reacts with a second ethylene. Eventually, the polyethylene is characterized by its repeating unit $+\mathrm{CH}_{2}-\mathrm{CH}_{2} \gamma_{x}$. In general, the center of activation at the end of the polymer may be a free radical, a carbocation (carbonium) or a carboanion, coining the terms radical, cationic or anionic polymerization. Other examples of addition polymerization which are used in gelation experiments are e.g. polymethylmethacrylate (PMMA) or cis-polyisoprene (natural rubber).

In contrast, condensation polymerization refers to processes where monomers react by eliminating by-products of low molecular weight, often water. Thus, the molecular formula of the repeating unit lacks certain atoms present in the monomer. An important example for the sol gel transition are silica gels, see Fig. 1.4. The reaction of another $\mathrm{Si}(\mathrm{OH})_{4}$ can occur at each of the hydroxyl groups leading to a nonlinear, branched topology of the network. A further example relevant for gelation are polyurethane gels.

As mentioned before, one particular polymerization process plays an important role for gelation: If the building blocks are themselves polymers of high molecular weight then the polymerization process is referred to as vulcanization. One distinguishes between end-linking, where solely endpoints of the chains are able to form (multiple) crosslinks and random crosslinking, where crosslinks can be established at different points along the contour length of the polymer. In these terms Goodyear's crosslinking of cis-polyisoprene 
by sulfur is classified as vulcanization by random crosslinking.

\subsubsection{Important experimental results}

Experiments on gelling systems have been done for a variety of chemical realizations by means of different techniques. The fundamental task is the determination of the gel point in order to measure quantities as a function of the distance $\varepsilon$ to the critical point. For an overview of detection methods see [WiMo97] and references therein. Then, the gelation process has to be quenched at a prescribed distance to the critical point. Alternatively, some authors perform continuous measurements in the course of time [AdDe85], and the extent of the reaction is then assumed to be proportional to the reaction time. The accuracy of the critical exponents is determined by the accuracy of the estimate of $\varepsilon$. Therefore, it is often advantageous to measure exponent ratios in order to get rid of the $\varepsilon$ dependence. For example, by measuring the viscosity as a function of the largest cluster size $n^{*}$ or the weight-averaged cluster size $n_{\mathrm{w}}$ the ratios $k / \sigma$ or $k / \gamma$ are obtained, respectively.

The first experiments on gelling systems focused on the determination of the cluster size distribution which is usually achieved by size-exclusion chromatography with subsequent low angle light scattering experiments (see [AdLa96] and references therein). Light scattering experiments provide a powerful method for investigating statics and dynamical properties of polymer solutions. A comprehensive overview is given in the textbook [Bro93]. However, light scattering experiments require the dilution of the gelling systems in order to produce a difference in molecular polarizability. As a consequence the fractal dimension $d_{\mathrm{f}}$ which is measured in the diluted state, and likewise other static and dynamic properties differ from those of the dense state due to the aforementioned swelling of the clusters. Thus, static and dynamic properties of the dense reaction bath cannot be accessed directly.

Rheological experiments are carried out in rheometers. The most prominent geometries for shear flows are rotational rheometers, such as parallel disks, concentric cylinders (Couette viscometer) or the cone-and-plate rheometer (see [BiCu87, Ch. 10]). In a rheometer the sample is subjected to a macroscopic, small-amplitude strain in order to avoid rupture of the fragile network. Usually, oscillatory strains are used which provide direct access to $\widehat{G}(\omega)$.

The major experimental results which have been obtained in the last two decades can be summarized as follows (see [AdLa96] and references therein).

1. It has been confirmed for a variety of chemical gelation processes, that the exponents which characterize the connectivity properties in gelling systems are well described by the predictions of three dimensional lattice percolation.

2. The fractal dimension of swollen percolation clusters is roughly given by $d_{\mathrm{f}} \approx 2$ and differs from the fractal dimension of percolation clusters. In one case the hyperscaling law $d_{\mathrm{f}} / d=\tau-1$ has been verified [AdLa91], which implies $d_{\mathrm{f}} \approx d_{\mathrm{f}}^{(\mathrm{H})}$, i.e. the Hausdorff fractal dimension is valid in the dense state. 
3. The scaling assumption for $\widehat{G}(\omega)$ has been verified in several cases [LuMo95, AxKo90, LuMo95].

4. Dynamic exponents scatter significantly. For instance the viscosity exponent scatters over a range of $[0.2 \ldots 1.5]$.

\subsection{Scope of this work}

\subsubsection{Motivation}

The agreement of theoretical predictions with experimental results is far from being satisfactory. Scaling arguments provide a powerful tool to understand fundamental aspects of statics and dynamics of gelling systems, but still bear some fundamental conceptual drawbacks. For example, the role of thermal fluctuations of macromolecules is completely neglected within an a priori application of geometric properties of percolation. It is by no means clear why the radius of gyration of a cluster in the reaction bath, which is subjected to thermal fluctuations and connectivity fluctuations, can be described by lattice percolation which only accounts for the connectivity fluctuations. Moreover, scaling theory is not able to answer particular questions concerning dynamical aspects of gelation such as the wide scatter of the exponent of the shear viscosity.

Therefore, in addition to scaling theory, it is useful to consider semi-microscopic models. Some striking features of the gelation transition have been revealed within a statistical mechanics approach of randomly crosslinked polymers which was introduced in [GoGo87, GoGo89a, GoGo89b] and explored in detail in [GoCa96]. For a less technical overview see [ZiGo97]. The model describes flexible, self-avoiding polymer chains which are subjected to a crosslinking mechanism which avoids an a priori imposition of percolation statistics. Crosslinks are rather established on the basis of nearest neighbor correlations of the monomers in the uncrosslinked fluid. This type of crosslink statistics was introduced by Deam and Edwards in [DeEd76, BaEd80] and is therefore referred to as Deam-Edwards distribution. It has the advantage of being easily incorporated into the field-theoretic treatment of the Edwards-Hamiltonian [DeEd76] of self-avoiding polymer chains. The advantage of this statistical mechanics approach is the possible analysis of thermal degrees of freedom in the presence of quenched degrees of freedom. An exact mean field theory reveals striking physical features which cannot be deduced from the percolation picture: At the critical point the system's symmetry of translational invariance is spontaneously broken due to the localization of a finite fraction of particles in space, the gel. The emergence of a nonzero shear modulus can be deduced quite generally from Goldstone excitations which are associated with the broken, continuous symmetry of translational invariance [MuGo04]. Moreover, a renormalization group approach reveals that those geometric properties which have a direct counterpart in percolation, e.g. the pair connectedness function, gel fraction etc lie in the percolation universality class [PeGo00, JaSt01, PeGo01]. 
In principle it would be desirable to have a dynamic formulation of the aforementioned model. Since the technical realization of a dynamic formulation is presently unfeasible, the present work starts from the simplest, semi-microscopic, dynamical models, the Rouse [Rou53] and the Zimm model [Zim56]. These models describe phantom rather than self avoiding macromolecules and provide the starting point of almost all dynamical theories of polymers, [DoEd88, $\mathrm{BiCu} 87]$.

\subsubsection{Outline}

In the next, the second chapter the general theoretical framework for a dynamical description of macromolecules in solution is provided. By specifying this general framework to phantom clusters the Rouse and Zimm model of gelation is obtained being the basis for the present work. The third chapter introduces relevant physical observables which are suitable to detect critical behavior of gelling systems. In the fourth chapter the solution of the equations of motion of the Rouse and the Zimm model is presented and the observables are computed within these models. The fifth chapter provides the methods which are employed to compute the critical behavior of the observables. The results are presented in the sixth chapter. In the last chapter, the results are summarized and discussed afterwards by referring to experiments and scaling theories which have been presented here in the introduction. Most results presented in this work have been published in [BrLo01a, BrLo01b, KuLo03, LoMu04]. 


\section{Chapter 2}

\section{Dynamical Model of gelling liquids}

\subsection{Static properties of macromolecules}

A suitable starting point for a semi-microscopic description of static configurations of polymers is the concept of a Gaussian macromolecule which is a coarse grained description, neglecting chemical details. Consider for instance a sketch of a realistic polymer, the so-called freely rotating chain (see Fig. 2.1) where the details of the chemical bonding between neighboring monomers is such that the bond $b_{s+1}$ is allowed to rotate freely around the bond $\boldsymbol{b}_{s}$ at a fixed angle $\vartheta$. In this case the orientational correlations between two bond vectors along the chain decay exponentially as a function of the number of bonds in between [DoEd88, Ch. 2.1.2]. Accordingly, if the number of chemical units, that is the chemical monomers between $\boldsymbol{R}_{1}$ and $\boldsymbol{R}_{2}$ is sufficiently large the extension $\left(\boldsymbol{R}_{2}-\boldsymbol{R}_{1}\right)$ between the endpoints is effectively described by a (locally stiff) random walk and thus determined by a Gaussian probability distribution. Equivalently, one may replace the subunits by an entropic spring with spring potential proportional to $k_{\mathrm{B}} T / a^{2}$. The parameter $a$, the persistence length covers the chemical details, i.e. $\vartheta$, the microscopic bond lengths and the number of microscopic subunits. The resulting mesoscopic unit is also known as a Kuhn segment (see Ch. 2 in [DoEd88] or Ch. 11 in [BiCu87]). The mesoscopic length scale of these segments is an intermediate scale between the size of a single chemical unit and and the size of the whole macromolecule.

As a consequence, on these mesoscopic length scales the interactions between monomers are described by effective interactions. Consider a collection of $N$ monomers which are characterized by their position vectors $\boldsymbol{R}_{i}, i=1, \ldots, N$, in three-dimensional Euclidean space $\mathbb{R}^{3}$. The potential energy $U(\{\boldsymbol{R}\})$ is then assumed to consist solely of two-body contributions. Here the shorthand notation $\{\boldsymbol{R}\}:=\left\{\boldsymbol{R}_{1}, \boldsymbol{R}_{2}, \ldots, \boldsymbol{R}_{N}\right\}$ for the configuration is used. A minimal model for macromolecules is the Edwards-Hamiltonian which assumes the potential energy to be composed of two different terms

$$
U(\{\boldsymbol{R}\})=U_{\text {con }}(\{\boldsymbol{R}\})+U_{\text {ev }}(\{\boldsymbol{R}\}) .
$$

The entropic, harmonic term $U_{\text {con }}(\{\boldsymbol{R}\})$ ensures that the molecules connectivity is preserved according to its coarse grained molecular topology. It applies to dimers, trimers, 


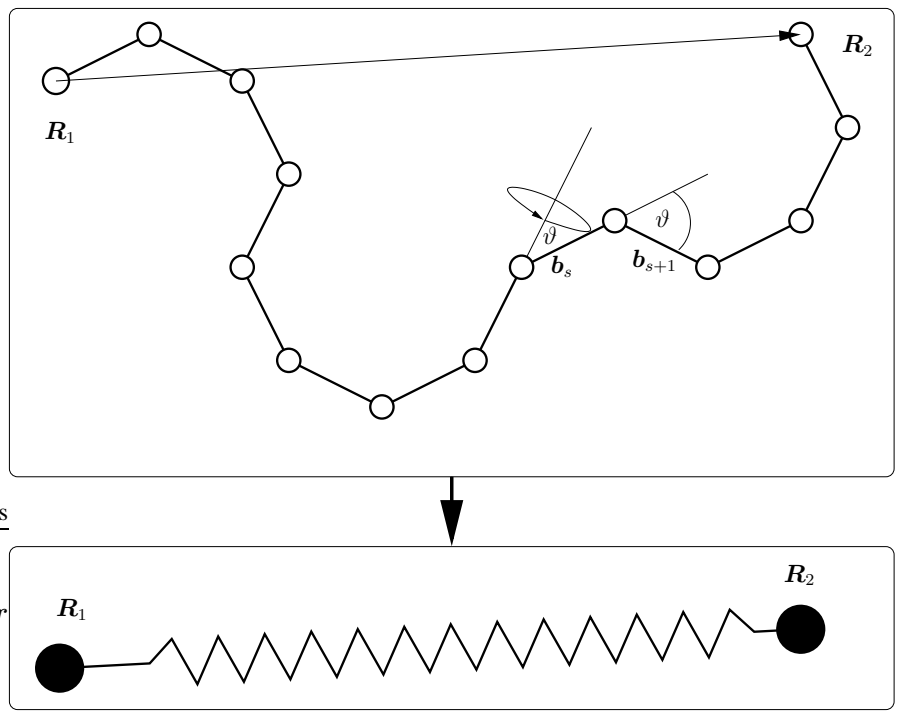

Figure 2.1: Coarse graining procedure for a segment of a "real" polymer, the freely rotating chain. A suffi ciently large number of monomers in the segment implies Gaussian statistics for the distance vector $\left(\boldsymbol{R}_{2}-\boldsymbol{R}_{1}\right)$ which is then replaced by an entropic spring.

linear chains, large crosslinked gelation clusters and all kinds of macromolecular topologies. The second term $U_{\text {ev }}(\{\boldsymbol{R}\})$ in (2.1) accounts for a short range repulsive interaction, the excluded volume interation which prevents monomers from occupying the same position in space.

\subsection{Dynamics of macromolecules in solution}

A common starting point for a dynamical description of macromolecules is to regard monomers as classical Brownian particles which are immersed in a solvent and interact via the potential (2.1). The solvent is described by the velocity field $\boldsymbol{u}(\boldsymbol{r}, t)$ and assumed to be incompressible which suffices to compute low frequency polymer dynamics. The motion of the fluid couples to the trajectories $\boldsymbol{R}_{i}(t)$ of the monomers by dissipative forces. Whenever the velocity of monomer $i$ deviates from the velocity $\boldsymbol{u}\left(\boldsymbol{R}_{i}(t), t\right)$ of the fluid at the monomer's position, it experiences a frictional force exerted by the solvent. This friction is characterized by a bare friction constant $\zeta$. For instance, if monomers were hard spheres, then the bare friction constant would be given by Stokes law $\zeta=6 \pi \eta_{\mathrm{s}} r_{\mathrm{m}}$ in terms of the solvent viscosity $\eta_{\mathrm{s}}$ and the radius of the sphere $r_{\mathrm{m}}$.

Here, the viscosity $\eta_{\mathrm{s}}$ of the solvent is supposed to be large, so that inertial forces on the monomers are small when compared to the frictional forces and therefore commonly neglected. In this case, the equation of motion for the monomers' positions is purely 
relaxational and given by

$$
0=-\zeta\left(\frac{\mathrm{d}}{\mathrm{d} t} \boldsymbol{R}_{i}(t)-\boldsymbol{u}\left(\boldsymbol{R}_{i}(t), t\right)\right)-\frac{\partial U(\{\boldsymbol{R}(t)\})}{\partial \boldsymbol{R}_{i}(t)}+\boldsymbol{\xi}_{i}(t)
$$

Here $\boldsymbol{\xi}_{i}(t)$ models the fluctuating Brownian force on monomer $i$ exerted by the solvent molecules. Usually, this random force is assumed to be Gaussian white noise with zero mean and co-variance

$$
\overline{\boldsymbol{\xi}_{i}(t) \boldsymbol{\xi}_{j}\left(t^{\prime}\right)^{\dagger}}=2 \zeta k_{\mathrm{B}} T \delta_{i, j} \delta\left(t-t^{\prime}\right) \mathbf{1}
$$

according to the fluctuation dissipation theorem. As usual, $\delta_{i, j}$ denotes the Kronecker symbol and $\delta\left(t-t^{\prime}\right)$ Dirac's delta function. The three-dimensional unit matrix is denoted by 1 .

The dynamical evolution of the velocity field $\boldsymbol{u}(\boldsymbol{r}, t)$ couples to the the monomers' positions due to the friction between particles and solvent. The presence of $N$ monomers give rise to additional stresses due to the presence of potential forces $\boldsymbol{F}_{i}(t)=$ $-\partial U(\{\boldsymbol{R}(t)\}) / \partial \boldsymbol{R}_{i}(t)$ on monomer $i$ at time $t$. The dynamics of the flow field is described by fluctuating hydrodynamics for an incompressible fluid which is governed by the Navier-Stokes equation [WaFr88, OeRa89]

$$
\begin{aligned}
& \rho_{\mathrm{s}}\left[\frac{\partial \boldsymbol{u}(\boldsymbol{r}, t)}{\partial t}+\boldsymbol{u}(\boldsymbol{r}, t) \nabla \boldsymbol{u}(\boldsymbol{r}, t)\right]=\eta_{\mathrm{s}} \Delta \boldsymbol{u}(\boldsymbol{r}, t)-\nabla p(\boldsymbol{r}, t) \\
& -\sum_{i=1}^{N} \frac{\partial U(\{\boldsymbol{R}(t)\})}{\partial \boldsymbol{R}_{j}(t)} \delta\left(\boldsymbol{r}-\boldsymbol{R}_{i}(t)\right)+\boldsymbol{f}(\boldsymbol{r}, t) .
\end{aligned}
$$

Here $\rho_{\mathrm{s}}$ and $\eta_{\mathrm{s}}$ denote the density and the viscosity of the fluid, respectively and $\Delta$ is the three dimensional Laplace operator. The gradient $\nabla$ of the pressure $p(\boldsymbol{r}, t)$ can be eliminated by the incompressibility condition $\nabla \cdot \boldsymbol{u}(\boldsymbol{r}, t)=0$. The fluctuating force field $\boldsymbol{f}(\boldsymbol{r}, t)$ is chosen to be a zero-mean, Gaussian field with second moment

$$
\overline{\boldsymbol{f}(\boldsymbol{r}, t) \boldsymbol{f}^{\dagger}\left(\boldsymbol{r}^{\prime}, t^{\prime}\right)}=-2 \eta_{\mathrm{s}} k_{\mathrm{B}} T \Delta \delta\left(\boldsymbol{r}-\boldsymbol{r}^{\prime}\right) \delta\left(t-t^{\prime}\right) \mathbf{1}
$$

The closed set of equations $(2.2,2.4)$ describe the coupled motion of monomers and solvent. For many applications it useful to allow for an external flow field $\boldsymbol{u}_{\text {ext }}(\boldsymbol{r}, t)$ in order to calculate the response of the coupled system to the external flow. The velocity field is assumed to be a solution of (2.4) in the absence of the monomers. The most important flow situation is a simple shear flow (see Fig. 2.6). The velocity $\boldsymbol{u}_{\text {ext }}(\boldsymbol{r}, t)$ is in the $r^{x_{-}}$ direction, increasing linearly with $r^{y}$, i.e.

$$
\boldsymbol{u}_{\mathrm{ext}}(\boldsymbol{r}, t):=\dot{\gamma}(t) \boldsymbol{r}, \quad \dot{\gamma}(t)=\left(\begin{array}{ccc}
0 & \dot{\gamma}(t) & 0, \\
0 & 0 & 0 \\
0 & 0 & 0
\end{array}\right) .
$$

Here, the velocity gradient tensor $\dot{\gamma}(t)$ is characterized by the time-dependent shear rate $\dot{\gamma}(t)$. 


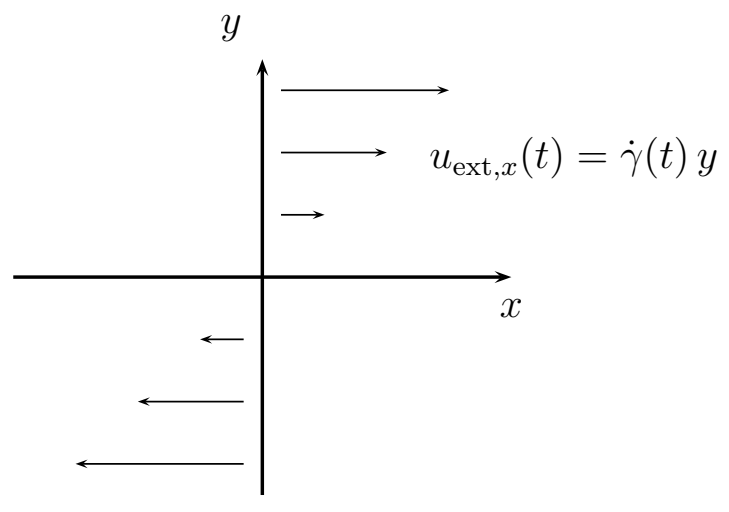

Figure 2.2: Simple shear fbw, characterized by the shear rate $\dot{\gamma}(t)$.

Since one is interested in dynamic properties of the macromolecules the coupled equations are condensed to a single equation for the polymers. This is, of course, only possible by employing some approximations. First, the nonlinear advection term $\boldsymbol{u}(\boldsymbol{r}, t) \nabla \boldsymbol{u}(\boldsymbol{r}, t)$ in (2.4) is omitted assuming a sufficiently large solvent viscosity and correspondingly low Reynolds number. The resulting linear equation for the velocity field can be solved by Fourier transformation, as shown in [OeRa89]. The solution as presented in detail in App. $\mathrm{A}$ is based on a separation of time scales, which is referred to as the Markovian assumption: The solvent relaxation times $\tau(k)=\rho_{\mathrm{s}} /\left(\eta_{\mathrm{s}} k^{2}\right)$ are assumed to be (infinitely) small when compared to the polymer relaxation times. This assumption indeed breaks down for relaxation times of perturbations on large spatial scales, corresponding to small $k$. However, the dynamics of the macromolecule is affected on spatial scales which ranges from the Kuhn length $a$ to the radius of gyration $R_{\text {gyr }}$. The solvent relaxation on the largest scale is given by $\tau_{s, \max }=\rho_{\mathrm{s}} R_{\mathrm{gyr}}^{2} / \eta_{\mathrm{s}}$. It will turn out, as already suggested by the discussion of the polymer relaxation time $t_{n}$ from Eq. (1.13) that the relaxation times on length scales $R_{\text {gyr }}$ are much larger. Even for the, less interesting, mesoscopic length scales which are associated with the persistence length $a$ of the Kuhn segment this separation of time scales still holds. For typical conditions, the (bare) relaxation time of the segment $\sim \zeta a^{2} /(k T)$ is still by a factor $10^{3}-10^{4}$ larger than the solvent relaxation time $\rho_{\mathrm{s}} a^{2} / \eta_{\mathrm{s}}$ [WaFr88]. Within the Markovian approximation the description of a polymer by the dynamical equations (2.2), (2.4) is equivalent (see e.g. [WaFr88, OeRa89]) to Kirkwood's kinetic theory approach [Kir54] to polymer dynamics which directly assumes Oseen like perturbations of the velocity field.

As a result of the linearity of the Navier-Stokes equation, the overall velocity field $\boldsymbol{u}(\boldsymbol{r}, t)(2.7)$ is a linear superposition of the applied external flow field $\boldsymbol{u}_{\text {ext }}(\boldsymbol{r}, t)$ and the 
contribution induced by the monomers which is given by the solution of (2.4) within the Markovian approximation. The validity of this linear superposition indeed requires weak flows, i.e. $\dot{\gamma} \ll \tau_{s \text {,max }}^{-1}$. Otherwise, the Oseen tensor is to be modified by terms stemming from the external flow [PuSc86]. Accordingly, the velocity field is given by

$$
\boldsymbol{u}(\boldsymbol{r}, t)=\boldsymbol{u}_{\mathrm{ext}}(\boldsymbol{r}, t)+\sum_{i=1}^{N} \Omega\left(\boldsymbol{r}-\boldsymbol{R}_{i}(t)\right) \boldsymbol{F}_{i}(t)+\boldsymbol{u}_{f}(\boldsymbol{r}, t)
$$

The monomer-induced perturbation of the external flow field in (2.7) is a mobility term, i.e. it depends linearly on the forces $\boldsymbol{F}_{i}$. It is characterized by the Oseen tensor [Ose10, KiRi48]

$$
\boldsymbol{\Omega}(\boldsymbol{r}):=\frac{1}{8 \pi \eta_{\mathrm{s}} r}\left(\mathbf{1}+\hat{\boldsymbol{r}} \hat{\boldsymbol{r}}^{\dagger}\right) .
$$

The Oseen tensor also determines the strength of the fluctuations of the random contribution $\boldsymbol{u}_{f}(\boldsymbol{r}, t)$ in (2.7) which has zero mean and covariance

$$
\left\langle\boldsymbol{u}_{f}(\boldsymbol{r}, t) \boldsymbol{u}_{f}\left(\boldsymbol{r}^{\prime}, t^{\prime}\right)\right\rangle=2 k_{\mathrm{B}} T \Omega\left(\boldsymbol{r}-\boldsymbol{r}^{\prime}\right) \delta\left(t-t^{\prime}\right) .
$$

The desired equation of motion for the polymers is now obtained by inserting (2.7) in (2.2). Unfortunately, the velocity field (2.7) evaluated at the position of monomer $i$ is infinite due to the singularity of the Oseen tensor. This hydrodynamic self interaction stems from the fact that particles are regarded as point-like objects which is in contradiction with the assumption of a finite frictional force between particles and solvent. Hydrodynamic self interactions are commonly avoided (see [DoEd88], Ch. 3.6) by forbidding the term $j=i$ when evaluating the velocity field (2.7) at the position $\boldsymbol{R}_{i}$ of monomer $i$, or equivalently, setting $\boldsymbol{\Omega}(\boldsymbol{r})=\mathbf{0}$.

It is convenient to define the mobility matrix

$$
\boldsymbol{H}_{i, j}(\boldsymbol{r}):=\delta_{i, j} \mathbf{1}+\left(1-\delta_{i, j}\right) \zeta \boldsymbol{\Omega}(\boldsymbol{r}) .
$$

by means of which the resulting stochastic differential equation is given by

$$
\zeta\left(\frac{\mathrm{d}}{\mathrm{d} t} \boldsymbol{R}_{i}(t)-\dot{\gamma}(t) \boldsymbol{R}_{i}\right)=-\sum_{j=1}^{N} \boldsymbol{H}_{i, j}\left(\boldsymbol{R}_{i}(t)-\boldsymbol{R}_{j}(t)\right) \frac{\partial U(\{\boldsymbol{R}(t)\})}{\partial \boldsymbol{R}_{i}(t)}+\boldsymbol{\eta}_{i}\left(\boldsymbol{R}_{i}, t\right)
$$

The random force field $\boldsymbol{\eta}_{i}(\boldsymbol{r}, t):=\boldsymbol{\xi}_{i}(t)+\zeta \boldsymbol{u}_{f}(\boldsymbol{r}, t)$ is a sum of two independent Gaussian white noises (see. App. A). It has zero mean and covariance

$$
\overline{\boldsymbol{\eta}_{i}(\boldsymbol{r}, t) \boldsymbol{\eta}_{j}^{\dagger}\left(\boldsymbol{r}^{\prime}, t^{\prime}\right)}=2 \zeta k_{\mathrm{B}} T \boldsymbol{H}_{i, j}\left(\boldsymbol{r}-\boldsymbol{r}^{\prime}\right) \delta\left(t-t^{\prime}\right)
$$

according to (2.3) and (2.9), which is consistent with the fluctuation dissipation theorem. Here the overbar indicates the average over all realizations of $\boldsymbol{\eta}$.

Equation (2.11) is the common starting point for studying dynamics of polymers in solution under the influence of an external flow (see Ch. 3,4 in [DoEd88], Ch. 15 in [BiCu87], 
Ch. 4 in [Oet96a]. It is a stochastic differential equation for the time evolution of the monomers' positions $\boldsymbol{R}_{i}(t)$ which is nonlinear due to the nonlinear dependence of the mobility matrix and the excluded volume interactions on the particles' positions.

It is often convenient to employ an alternative formulation of the Langevin dynamical equation (2.11): the equivalent Fokker-Planck equation for the probability density $p(\{\boldsymbol{R}\}, t)$ of finding the configuration $\{\boldsymbol{R}\}$ at time $t$, provided the system was in the configuration $\left\{\boldsymbol{R}_{0}\right\}$ at time $t_{0}$ [Oet96a, Ch. 3.3.3]. Here, the common usage is adopted and the equation for the time evolution of the probability density $p$ for the positions is commonly referred to as Smoluchowski equation, whereas the term Fokker-Planck equation is reserved for an equation for positions and momenta. The Smoluchowski equation is given by (see Eq. (3.121) in [DoEd88])

$$
\begin{aligned}
& \frac{\partial p(\{\boldsymbol{R}\}, t)}{\partial t}=-\sum_{j=1}^{N} \frac{\partial}{\partial \boldsymbol{R}_{j}} \cdot \dot{\gamma}(t) \boldsymbol{R}_{j} p(\{\boldsymbol{R}\}, t) \\
& \quad+\frac{1}{\zeta} \sum_{i, j=1}^{N} \frac{\partial}{\partial \boldsymbol{R}_{i}} \cdot \boldsymbol{H}_{i, j}\left(\boldsymbol{R}_{i}-\boldsymbol{R}_{j}\right)\left[k_{\mathrm{B}} T \frac{\partial p(\{\boldsymbol{R}\}, t)}{\partial \boldsymbol{R}_{i}}+p(\{\boldsymbol{R}\}, t) \frac{\partial U(\{\boldsymbol{R}\})}{\partial \boldsymbol{R}_{i}}\right] .
\end{aligned}
$$

There is a well known problem related to Eqs. (2.11), (2.13). The left hand side in (2.12) is by definition a positive matrix. However, the Oseen tensor does not give rise to a positive-definite mobility matrix $\boldsymbol{H}_{i, j}\left(\boldsymbol{R}_{i}-\boldsymbol{R}_{j}\right)$ for all possible spatial configurations of monomers [ArJh81]. For configurations where monomers are very close to each other the mobility matrix gives rise to negative eigenvalues. This is a serious problem since a covariance matrix being not positive definite is physically meaningless. Likewise, the problem arises in numerical integration schemes of the Langevin equation (2.11) since stability of these Brownian dynamics algorithms rely on the positive definiteness of $\boldsymbol{H}_{i, j}\left(\boldsymbol{R}_{i}-\boldsymbol{R}_{j}\right)$ for all $\{\boldsymbol{R}\}$. In numerical applications this problem is commonly circumvented by using a different hydrodynamic interaction tensor instead, the Rotne-Prager tensor [RoPr69, Yam70]. The derivation of the tensor [RoPr69] is based on a variational principle for the energy dissipation rate of a sphere with finite radius $r_{\mathrm{m}}$ due to the motion of the suspending fluid. As a result, the Rotne-Prager tensor

$$
\boldsymbol{\Omega}(\boldsymbol{r})=\frac{1}{8 \pi \eta_{\mathrm{s}} r} \begin{cases}{\left[1+\frac{1}{6}\left(\frac{2 r_{\mathrm{m}}}{r}\right)^{2}\right] \mathbf{1}+\left[1-\frac{1}{2}\left(\frac{2 r_{\mathrm{m}}}{r}\right)^{2}\right] \hat{\boldsymbol{r}} \hat{\boldsymbol{r}}^{\dagger},} & r \geq 2 r_{\mathrm{m}} \\ {\left[\frac{8}{3}\left(\frac{r}{2 r_{\mathrm{m}}}\right)-\frac{3}{2}\left(\frac{r}{2 r_{\mathrm{m}}}\right)^{2}\right] \mathbf{1}+\frac{1}{2}\left(\frac{r}{2 r_{\mathrm{m}}}\right)^{2} \hat{\boldsymbol{r}} \hat{\boldsymbol{r}}^{\dagger},} & r<2 r_{\mathrm{m}}\end{cases}
$$

is shown to be a positive definite approximation to the true hydrodynamic interaction tensor for all configurations $\{\boldsymbol{R}\}$. If two monomers are at a large distance, the expression (2.14) coincides with the Oseen tensor (2.8) for $r / r_{\mathrm{m}} \rightarrow \infty$, whereas for configurations $r \lesssim 2 r_{\mathrm{m}}$ in which monomers are very close to each other the singular behavior of the Oseen tensor is regularized in (2.14).

Even within the approximations made so far, the resulting equation of motion (2.11) is still analytically hardly feasible due to the nonlinear dependence of the hydrodynamic and 
excluded volume interactions on the monomers' positions. For a single, regular connected, self-avoiding macromolecule such as a linear polymer or a two dimensional membrane Eq. (2.11) can be addressed analytically by means of a renormalization group approach [Wie98a, Wie98b]. In the next sections the most important approximations to equation (2.11) are discussed.

\subsection{Dynamic model of phantom clusters}

The most important and simplest approximations of Eq. (2.11), namely the Rouse and the Zimm model [Rou53, Zim56] are obtained by neglecting excluded volume interactions. In the absence of excluded volume interactions the polymers are said to be ideal or phantom-like. This appears to be a crude approximation but for linear polymers it has been recognized that there are indeed specific experimental situations where the polymers behave ideally. First, in dense melts the excluded volume interaction is screened [Gen79a, Ch. II.1]. Second, if the polymers are immersed in a good solvent it is possible to tune the quality of the solvent and thereby the second virial coefficient of monomer-monomer interactions. The temperature where the second virial coefficient vanishes is referred to as theta temperature [DoEd88, Ch. 2.5].

Before turning to the dynamics of phantom clusters, it is helpful to examine the characteristics of static properties of phantom clusters first.

\subsubsection{Statics of phantom clusters}

Without excluded volume interactions the potential energy (2.1) consists solely of the connectivity term. The connectivity of the macromolecules is guaranteed by permanently formed bonds or crosslinks which constrain $M$ randomly chosen pairs of particles $\left(i_{e}, j_{e}\right)$, $e=1, \ldots, M$. Crosslinks are modeled by entropic Hookean springs, giving rise to a potential energy

$$
U(\{\boldsymbol{R}\}):=\frac{3 k_{\mathrm{B}} T}{2 a^{2}} \sum_{e=1}^{M}\left(\boldsymbol{R}_{i_{e}}-\boldsymbol{R}_{j_{e}}\right)^{2}=: \frac{3 k_{\mathrm{B}} T}{2 a^{2}} \sum_{i, j=1}^{N} \boldsymbol{R}_{i} \cdot \Gamma_{i, j} \boldsymbol{R}_{j} .
$$

The persistence length $a>0$ plays the role of an inverse crosslink strength. A given crosslink configuration $\mathscr{G}=\left\{i_{e}, j_{e}\right\}_{e=1}^{M}$ which can be regarded as a graph is specified by its $N \times N$-connectivity matrix $\Gamma(\mathscr{G})$. The matrix elements are indexed by the monomers or vertices of the graph $\mathscr{G}$. In graph theory the matrix $\Gamma$ is referred to as Laplacian matrix (see e.g. Ch. 13 in [GoRo01]). The Laplacian matrix is the difference between the diagonal matrix of vertex degrees and the adjacency matrix of the graph. The off diagonal elements $\Gamma_{i, j}$ are equal to -1 if monomers $i$ and $j$ are adjacent, i.e. connected by a crosslink or $e d g e$ and zero otherwise. The diagonal element $\Gamma_{i, i}$ is the vertex degree of monomer $i$, i.e. the 
number of crosslinks or edges emanating from monomer $i$. One has

$$
\Gamma_{i, j}=\left\{\begin{array}{ll}
-1, & i \neq j, \text { and } i \text { adjacent to } j \\
-\sum_{k \neq i, k=1}^{N} \Gamma_{i, k}, & i=j
\end{array} .\right.
$$

and hence by definition the row and column sums of $\Gamma$ vanish, $\sum_{j=1}^{N} \Gamma_{i, j}=0$. The simplest example of a connectivity matrix is provided by the linear polymer chain consisting of $n$ monomers

$$
\Gamma\left(\mathscr{P}_{n}\right)=\left(\begin{array}{ccccc}
1 & -1 & 0 & \ldots & 0 \\
-1 & 2 & -1 & & \vdots \\
0 & & \ddots & & 0 \\
\vdots & & -1 & 2 & -1 \\
0 & \ldots & 0 & -1 & 1
\end{array}\right) .
$$

The matrix (2.17) is nothing but the discretized Laplacian operator on a finite interval on $\mathbb{R}$ with Neumann boundary conditions.

The connectivity matrix $\Gamma$ is of major importance in the Rouse model. Therefore it is necessary to examine some of its mathematical properties and their physical relevance. First of all, it is a positive semi-definite matrix, which follows directly from the nonnegative potential energy (2.15). The zero eigenvalues of $\Gamma$ stem from the symmetry of $U(\{\boldsymbol{R}\})$ under translations of individual clusters, that is its maximal pathwise connected components. This is a consequence of pair interactions in (2.15), which imply that no forces act on the centers of mass of individual clusters. To clarify this connection between the centers of mass of the clusters and the zero eigenvalues of $\Gamma$, the graph $\mathscr{G}$ is decomposed into its clusters according to

$$
\mathscr{G}=\bigcup_{k=1}^{K} \mathscr{N}_{k}
$$

Hence, the connectivity matrix is block diagonal

$$
\Gamma(\mathscr{G})=\bigoplus_{k=1}^{K} \Gamma\left(\mathscr{N}_{k}\right)
$$

where one block is the connectivity matrix of one cluster. A simple example of the cluster decomposition and the used notation is given in Fig. 2.3.1. The size of a block $\Gamma\left(\mathscr{N}_{k}\right)$ is equal to the number $N_{k}$ of monomers in the $k^{\text {th }}$ cluster $\mathscr{N}_{k}$, where the cluster sizes must add up to $\sum_{k=1}^{K} N_{k}=N$. Furthermore it is convenient to denote the cluster of monomer $i$ by $\mathscr{N}(i)$ and its size by $N(i)$.

The subspace of zero eigenvalues is spanned by the vectors which are constant when restricted to any one cluster since the row sums of $\Gamma$ vanish according to (2.16). Hence, the number of zero eigenvalues, i.e. the dimension of the null space of $\Gamma$, is equal to the number of clusters $K$. Denoting by $E_{0}\left(\mathscr{N}_{k}\right)$ the projector onto the null space of the connectivity 


$$
\mathscr{G}=\mathscr{N}_{1} \cup \mathscr{N}_{2}
$$

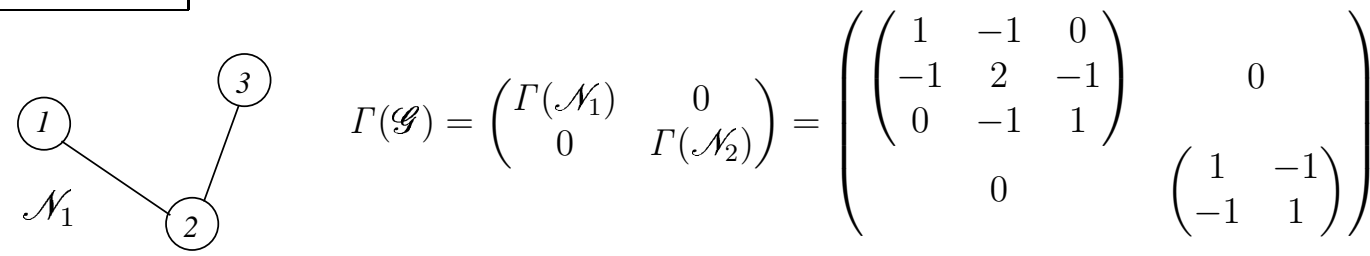

$$
\begin{aligned}
& \mathscr{N}_{2}
\end{aligned}
$$

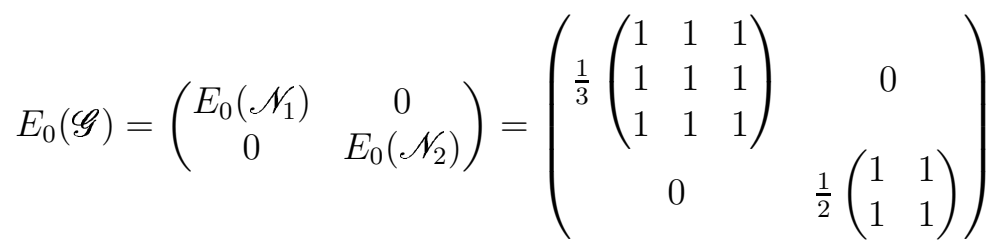

Figure 2.3: Example of the cluster decomposition and the associated notation. On the left, a graph or crosslink confi guration $\mathscr{G}$ with $N=5$ monomers or vertices, is decomposed into its $K=2$ clusters $\mathscr{N}_{1}, \mathscr{N}_{2}$ with cluster sizes $N_{1}=3$ and $N_{2}=2$. The connectivity matrix $\Gamma(\mathscr{G})$ consists of two blocks, $\Gamma\left(\mathscr{N}_{1}\right)$ and $\Gamma\left(\mathscr{N}_{2}\right)$ which are the connectivity matrices of the clusters. In this example $\Gamma(\mathscr{G})$ has $K=2$ zero eigenvalues and the corresponding projector $E_{0}(\mathscr{G})$ onto the null space is given. It is easy to see that $\Gamma(\mathscr{G}) E_{0}(\mathscr{G})=0$.

matrix $\Gamma\left(\mathscr{N}_{k}\right)$ of a cluster its matrix element are given explicitly by

$$
\left[E_{0}\left(\mathscr{N}_{k}\right)\right]_{i, j}=\frac{1}{N_{k}}
$$

(see App. B.1). Therefore, the center of mass of the cluster of monomer $i$, that is $\mathscr{N}(i)$, can be written in the following form

$$
\boldsymbol{R}_{\mathrm{cm}}(\mathscr{N}(i))=\sum_{j=1}^{N}\left[E_{0}(\mathscr{G})\right]_{i, j} \boldsymbol{R}_{j}
$$

It will be helpful to keep in mind that the projector $E_{0}$ is related to the centers of mass.

The connectivity matrix completely determines the static conformations of phantom clusters via the potential energy $(2.15)$ in the Boltzmann weight $\sim e^{-U(\{\boldsymbol{R}\}) / k_{\mathrm{B}} T}$. However, in the computation of equilibrium averages care has to be taken of the zero eigenvalues of $\Gamma$. To this end the potential (2.15) is regularized by adding a confining term $3 \omega /\left(2 a^{2}\right) \sum_{i=1}^{N} \boldsymbol{R}_{i} \cdot \boldsymbol{R}_{i}$ with $\omega>0$ and taking the limit $\omega \downarrow 0$ subsequently. In this way it is shown in App. D.1 that the distribution of mutual distance vectors $\left\langle\left(\boldsymbol{R}_{i}-\boldsymbol{R}_{j}\right)\right\rangle_{\text {eq }}$ of monomers $i$ and $j$ within the same clusters is a Gaussian distribution

$$
\begin{aligned}
p_{i, j}(\boldsymbol{r}): & =\left\langle\delta\left(\left(\boldsymbol{R}_{i}-\boldsymbol{R}_{j}\right)-\boldsymbol{r}\right)\right\rangle_{\mathrm{eq}} \\
& =\left(\frac{3}{2 \pi \mathscr{R}_{i, j} a^{2}}\right)^{3 / 2} \exp \left\{-\frac{3 \boldsymbol{r}^{2}}{2 \mathscr{R}_{i, j} a^{2}}\right\} .
\end{aligned}
$$

It is characterized by the mean squared distance

$$
\mathscr{R}_{i, j}:=\left[\frac{\mathbb{1}-E_{0}}{\Gamma}\right]_{i, i}+\left[\frac{\mathbb{1}-E_{0}}{\Gamma}\right]_{j, j}-2\left[\frac{\mathbb{1}-E_{0}}{\Gamma}\right]_{i, j}
$$


which is determined by the matrix elements of the Moore-Penrose inverse $\left(\mathbb{1}-E_{0}\right) / \Gamma$ of the connectivity matrix, where $\mathbb{1}$ denotes the $N$-dimensional unit matrix. The MoorePenrose inverse is a particular pseudo- or generalized inverse of a matrix [Alb72] which is uniquely defined even for singular matrices. The Moore-Penrose inverse of $\Gamma$ is the inverse restricted to the subspace of nonzero eigenvalues which is most easily revealed by the spectral decomposition of $\Gamma$ (App. B.1).

Using the pair distribution (2.22), it is then straightforward to compute static properties, such as the squared radius of gyration of a cluster $\mathscr{N}_{k}$. It is given by

$$
R_{\mathrm{gyr}}^{2}\left(\mathscr{N}_{k}\right):=\left\langle\frac{1}{2 N_{k}^{2}} \sum_{i, j \in \mathscr{N}_{k}}\left(\boldsymbol{R}_{i}-\boldsymbol{R}_{j}\right)^{2}\right\rangle_{\text {eq }}=\frac{a^{2}}{2 N_{k}^{2}} \sum_{i, j \in \mathscr{N}_{k}} \mathscr{R}_{i, j}
$$

and hence characterized by the Moore-Penrose inverse of $\Gamma$ via (2.24). After this brief intermezzo of static properties of phantom clusters the dynamics is addressed in the next section.

\subsubsection{Rouse model: Free draining approximation}

In the Rouse model [Rou53] hydrodynamic interactions are neglected. Formally, this is achieved by setting $\boldsymbol{H}_{i, j}=\delta_{i, j} \mathbf{1}$ in (2.11. This means that the velocity field $\boldsymbol{u}(\boldsymbol{r}, t)$ is not perturbed by the monomers and simply given by the externally imposed velocity field $\boldsymbol{u}_{\text {ext }}(\boldsymbol{r}, t)(2.6)$. These conditions are most likely met in dense melts in the absence of a solvent.

Both, the simple shear flow (2.6) and the gradient of the potential energy (2.15) are linear functions of the coordinates $\{\boldsymbol{R}\}$, hence the equation of motion reduces to the linear, stochastic differential equation

$$
\zeta\left(\frac{\mathrm{d}}{\mathrm{d} t} \boldsymbol{R}_{i}(t)-\dot{\gamma}(t) \boldsymbol{R}_{i}\right)=-\sum_{j=1}^{N} \frac{\partial U(\{\boldsymbol{R}\})}{\partial \boldsymbol{R}_{i}(t)}+\boldsymbol{\xi}_{i}(t) .
$$

The co-variance of the white noise is now given by

$$
\overline{\boldsymbol{\xi}_{i}(t) \boldsymbol{\xi}_{j}^{\dagger}\left(t^{\prime}\right)}=2 \zeta k_{\mathrm{B}} T \delta_{i, j} \delta\left(t-t^{\prime}\right) \mathbf{1}
$$

In the absence of hydrodynamic interactions all monomers exhibit the same frictional force, independent of their positions within the macromolecule. The cluster is said to be freely drained by the flow. Individual clusters move independently in the Rouse model which follows from the block diagonal structure of $\Gamma$.

\subsubsection{Zimm model: Preaveraged hydrodynamic interactions}

In dilute solution it is important to account for the influence of the solvent. But even in the absence of excluded volume interactions the incorporation of hydrodynamic interactions in 
equation (2.11) is analytically unfeasible due to the nonlinear dependence of the mobility matrix on the monomers' positions. The simplest approximation which can be applied to (2.11) is the so-called preaveraging approximation. This was first introduced by Kirkwood and Risemann [KiRi48] and Zimm [Zim56]. Within the preaveraging approximation the mobility matrix (2.10) is replaced by its equilibrium expectation value, which is computed with the equilibrium distribution function, the Boltzmann weight $\sim e^{-U(\{\boldsymbol{R}\}) / k_{\mathrm{B}} T}$. Due to rotational invariance of the potential energy $(2.15)$ the preaveraged mobility matrix $(2.10)$ is a multiple of the identity matrix

$$
\begin{aligned}
\left\langle\boldsymbol{H}_{i, j}\left(\boldsymbol{R}_{i}-\boldsymbol{R}_{j}\right)\right\rangle_{\mathrm{eq}} & =\delta_{i, j} \mathbf{1}+\left(1-\delta_{i, j}\right) \zeta\left\langle\boldsymbol{\Omega}\left(\boldsymbol{R}_{i}-\boldsymbol{R}_{j}\right)\right\rangle_{\mathrm{eq}} \\
& =: H_{i, j} \mathbf{1}
\end{aligned}
$$

This result is independent of the choice of the hydrodynamic interaction tensor $\Omega\left(\boldsymbol{R}_{i}-\right.$ $\left.\boldsymbol{R}_{j}\right)$. As presented in App. D.2 the equilibrium average of $\Omega\left(\boldsymbol{R}_{i}-\boldsymbol{R}_{j}\right)$ for the Oseen- (2.8) and the Rotne-Prager tensor (2.14) can be computed by using the pair distribution (2.22). As a result, the preaveraged mobility matrix $H$ shows correlations of different particles only if these particles are in the same cluster, in other words $H$ is block-diagonal. Within one block or cluster, the matrix element $H_{i, j}$ depends only on the mean squared distance $\mathscr{R}_{i, j}$ between monomers $i$ and $j$ and is given by

$$
H_{i, j}=\delta_{i, j}+\left(1-\delta_{i, j}\right) h\left(\frac{\kappa^{2} \pi}{\mathscr{R}_{i, j}}\right) .
$$

The choice of the function $h$ depends on the choice of the hydrodynamic interaction tensor. For the Oseen tensor one has

$$
h(x)=\sqrt{x / \pi}
$$

whereas for the Rotne-Prager tensor (cf. [Fix83b])

$$
h(x)=\operatorname{erf}(x)-\left(1-\exp \left(-x^{2}\right) /\left(\pi^{1 / 2} x\right)\right) .
$$

The latter involves the error function $\operatorname{erf}(\mathrm{x})$ and reduces to the former as $x \downarrow 0$.

The preaveraged mobility matrix (2.29) involves a parameter

$$
\kappa:=\sqrt{\frac{6}{\pi}} \frac{\zeta}{6 \pi \eta_{\mathrm{s}} a}
$$

which plays the role of the coupling constant of the hydrodynamic interactions. Formally setting $\kappa=0$ in (2.29) yields $H_{i, j}=\delta_{i, j}$, and the Zimm model for gelation reduces to the Rouse model for crosslinked monomers (2.26) [BrGo97, BrLo99, BrLo01a].

By replacing the mobility matrix in (2.11) with the preaveraged mobility matrix (2.29), the Zimm model for crosslinked monomers in solution is obtained

$$
\zeta\left(\frac{\mathrm{d}}{\mathrm{d} t} \boldsymbol{R}_{i}(t)-\dot{\gamma}(t) \boldsymbol{R}(t)\right)=-\sum_{j=1}^{N} H_{i, j} \frac{\partial U}{\partial \boldsymbol{R}_{j}(t)}+\boldsymbol{\eta}_{j}(t) .
$$


The co-variance of the thermal noise is then characterized by

$$
\overline{\boldsymbol{\eta}_{i}(t) \boldsymbol{\eta}_{j}^{\dagger}\left(t^{\prime}\right)}=2 k_{\mathrm{B}} T \zeta H_{i, j} \delta\left(t-t^{\prime}\right) \mathbf{1}
$$

The Zimm equation (2.33) is linear, hence it can be solved exactly. Since the connectivity matrix $\Gamma$ as well as the mobility matrix $H$ are block-diagonal, it follows that clusters move independently, since there is no force on the center of mass of a cluster. However, in the presence of hydrodynamic interactions, it is not the center of mass which diffuses freely, it is rather the hydrodynamic center of resistance ([Oet96a] Ch. 4.2.2, [BiCu87] Ch. 15.4)

$$
\boldsymbol{R}_{\mathrm{cr}}:=\frac{\sum_{i, j}\left[H^{-1}\right]_{i, j} \boldsymbol{R}_{i}}{\operatorname{Tr}\left(E_{0} H^{-1}\right)} .
$$

It is defined such that it coincides with the center of mass when hydrodynamic interactions are neglected.

For the sake of completeness it is pointed out that a different preaveraging procedure has been proposed by Öttinger, namely the self consistent averaging [Oet87a] method. The mobility matrix $\boldsymbol{H}(\boldsymbol{r})$ in $(2.11)$ is replaced by an a priori unknown mobility matrix $\boldsymbol{H}^{\text {av }}$ which is independent of $\boldsymbol{r}$. This yields a solution $\boldsymbol{R}_{i}(t)$ of the resulting linear equation which is a function of the unknown matrix elements of $\boldsymbol{H}^{\text {av }}$. The mobility matrix is then determined a posteriori self consistently from the condition that $\boldsymbol{H}_{i, j}^{\text {av }}$ is equal to the average of $\boldsymbol{H}\left(\boldsymbol{R}_{i}(t)-\boldsymbol{R}_{j}(t)\right)$ over the realizations of the solution $\boldsymbol{R}_{i}(t)$. As a result $\boldsymbol{H}^{\text {av }}$ depends on the shear rate. The zeroth order contribution agrees with the equilibrium preaveraged $H 1$ of the Zimm model. The incorporation of higher order corrections to the Zimm approximation becomes important, if shear rate dependent material functions are considered. It is common to all preaveraging procedures that the resulting equations are linear and can thus be solved exactly.

\subsection{Crosslink statistics}

\subsubsection{Disorder averages}

In order to determine the dynamic models of phantom clusters completely, the probability distribution $P(\mathscr{G})$ of crosslinks configurations $\mathscr{G}$ needs to be specified. All observables $A(\mathscr{G})$ considered throughout this work are expected to be self-averaging quantities, that is, in the macroscopic limit they are independent of the realization of the crosslink configuration. However, from a technical point of view it is advantageous to compute the average of observables over the probability distribution in the macroscopic limit which is denoted by

$$
A:=\langle A(\cdot)\rangle:=\sum_{\mathscr{G}} P(\mathscr{G}) A(\mathscr{G}) .
$$

The average $\langle\bullet\rangle$ includes the macroscopic limit $M \rightarrow \infty, N \rightarrow \infty$ with fixed crosslink concentration $c:=M / N$. 
Experimental results suggest that three dimensional percolation is an adequate choice for characterizing the probability distribution $P(\mathscr{G})$. Here, in fact any percolation ensemble could be considered which is amenable to a scaling description in the vicinity of the percolation threshold, giving rise to a cluster size distribution

$$
\tau_{n} \sim n^{-\tau} f\left(-n / n^{*}\right)
$$

for $\varepsilon \ll 1$ and $n \rightarrow \infty$ with a typical cluster size $n^{*}(\varepsilon) \sim \varepsilon^{-1 / \sigma}$ that diverges as $\varepsilon \rightarrow 0$. Here, $\varepsilon=\left|c-c_{\text {crit }}\right| / c_{\text {crit }}$ denotes the distance to the critical point. Special attention is turned to two crosslink ensembles, three dimensional bond percolation and Erdős-Rényi random graphs which is a mean field distribution of crosslinks lacking any correlations between crosslinks.

\subsubsection{Crosslink distributions}

\subsubsection{Bond Percolation}

The most interesting ensemble is bond percolation on the three dimensional cubic lattice $\mathbb{Z}^{3}$ as defined in Sec. 1.2.2. Nearest-neighbor bonds are present with probability $p:=c /(2 d)$ and absent with probability $1-p$. The bonds are interpreted as crosslinks, while the lattice points are identified with monomers. Here, the probability $p$ is defined such that the average number of crosslinks is given by $M=c N$.

Note that only connectivity properties of lattice percolation are adopted by requiring that the scaling relations in Tab. 1.1, which characterize the connectivity without referring to spatial arrangement, remain valid. In contrast, scaling properties which describe the spatial correlations in three dimensional Euclidean space are not given by $(1.7,1.1)$. Spatial correlations are rather determined by the interactions (2.15) and the dynamical evolution (2.11).

From a different point of view, one may think of a preparation state which somehow guarantees that the crosslink distribution is chosen according to three dimensional bond percolation. The physics of the preparation of the clusters is not accounted for in this approach, only the output of the preparation is used for further analysis.

\subsubsection{Erdős-Rényi random graphs}

In the simplest distribution all pairs of monomers are equally likely to be crosslinked and no correlations between crosslinks are taken into account. Identifying the monomers and crosslinks with the vertices and edges of a graph, the statistical properties of such clusters have been studied extensively in the theory of random graphs, as developed by Erdős and Rényi in [ErRe60]. A survey of random graphs is given in the textbook [Bol85].

Strictly speaking, the ensemble of Erdôs-Rényi random graphs [ErRe60] is the ensemble of labelled graphs consisting of $N$ vertices, $M=c N$ edges and each of the $\left(\begin{array}{c}\left(\begin{array}{c}N \\ 2\end{array}\right) \\ M\end{array}\right)$ possible graphs with the same likelihood of occurence. In principle, this implies that multiple edges between two vertices or self-loops are forbidden. However, this restriction may 
be dropped in the macroscopic limit. This may be understood by comparing the number $\mathscr{O}(N)$ of possibilities to realize a self-loop or double edge in $\mathscr{G}$ with the number $\mathscr{O}\left(N^{2}\right)$ of possibilities to choose a different monomer.

This ensemble of random graphs exhibits a percolation transition at $c=c_{\text {crit }}=1 / 2$. For $c<c_{\text {crit }}$ there are almost surely no clusters with an extensive number of monomers in the macroscopic limit. All monomers belong to tree clusters without loops, see Thms. 5d,e in Ref. [ErRe60]. Moreover, according to Eq. (2.18) in Ref. [ErRe60], the cluster size distribution, that is the average number of trees of size $n$ per monomer is exactly given by

$$
\tau_{n}=\frac{n^{n-2}}{2 c n !}\left(2 c \mathrm{e}^{-2 c}\right)^{n}
$$

By applying Stirling's formula for large $n$, Eq. (2.38) can be rewritten according to

$$
\tau_{n} \sim \frac{1}{2 c \sqrt{2 \pi}} n^{-5 / 2} \mathrm{e}^{-n / n_{\mathrm{ER}}^{*}(c)},
$$

with $n_{\mathrm{ER}}^{*}(c):=(2 c-1-\ln (2 c))^{-1}$. This implies $n_{\mathrm{ER}}^{*}(c) \sim\left(c_{\text {crit }}-c\right)^{-2}$ for $c \uparrow c_{\text {crit }}$. Thus, Erdős-Rényi random graphs resemble the scaling properties of mean field percolation on a $d$-dimensional hypercubic lattice for $d \geq 6$. In contrast to the latter, it has the advantage that analytic expressions for the cluster size distribution are known. 


\section{Chapter 3}

\section{Dynamical signatures of the gelation transition}

\subsection{Stress relaxation}

\subsubsection{Stress tensor}

Quite generally, the rheological properties of fluids are determined by the constitutive equation which enables to compute the stress tensor (field) $\sigma_{\alpha, \beta}(\boldsymbol{r}, t)$ for a given velocity gradient tensor (field) $\partial_{\alpha} u_{\beta}(\boldsymbol{r}, t)$, where $\alpha, \beta=x, y, z$. The constitutive equation serves as the closure condition for the Navier-Stokes equation. For Newtonian liquids the constitutive equation is simply given by Newton's law of viscosity which assumes a linear relationship between the stress and the gradient of the velocity field

$$
\sigma_{\alpha, \beta}(\boldsymbol{r}, t)=-p \delta_{\alpha, \beta}+\eta_{\mathrm{s}}\left(\partial_{\alpha} u_{\beta}(\boldsymbol{r}, t)+\partial_{\beta} u_{\alpha}(\boldsymbol{r}, t)\right) .
$$

Here, $\eta_{\mathrm{s}}$ and $p$ denote the shear viscosity and the pressure of the the fluid, respectively. Note, that Eq. (3.1) is not the most general linear relation between two tensors of rank two. It pertains to incompressible fluids where the dilatational viscosity vanishes [LaLi59, Ch. II ]. In the following exclusively homogeneous flows are considered, that is, the velocity gradient tensor and accordingly the stress tensor are assumed to be independent of spatial position $r$.

In the presence of macromolecules the constitutive equation (3.1) for simple fluids fails. The fluids are referred to as complex fluids and the determination of the constitutive equation is rather difficult since the macroscopic stress at time $t$ may depend on higher order derivatives of the velocity field $\boldsymbol{u}(\boldsymbol{r}, t)$ and it may also depend on the complete history of the velocity gradient tensor for times $t^{\prime}<t$. If the flow field is specified to be the simple shear flow $u_{x}(t)=\dot{\gamma} y$ the stress tensor must have the following form

$$
\boldsymbol{\sigma}=-p \mathbf{1}+\left(\begin{array}{ccc}
\sigma_{x, x} & \sigma_{x, y} & 0 \\
\sigma_{x, y} & \sigma_{y, y} & 0 \\
0 & 0 & \sigma_{z, z}
\end{array}\right)
$$


This is a consequence of the isotropy and the invariance with respect to $180^{\circ}$-rotations about the $z$-axis. A further simplification can be made. In incompressible fluids the variation of pressure has no influence on the dynamics of the fluid. Therefore, isotropic contributions to the stress can always be subsumed in the pressure term. As a consequence, beside the uninteresting isotropic contribution, there remain only three independent quantities in (3.2), which characterize the stress tensor of an incompressible fluid in shear flow completely. These material functions are the shear stress $\sigma_{x, y}$ and the first and second normal stress differences $\sigma_{x, x}-\sigma_{y, y}$ and $\sigma_{y, y}-\sigma_{z, z}$. These quantities may depend nonlinearly on the shear rate $\dot{\gamma}$. The dependence of the material functions on the shear rate gives rise to peculiar rheological behavior of complex fluids, such as shear thinning, which refers to the generally observed decrease of the shear viscosity with increasing shear rate.

For vanishing shear rate, the material functions vanish since in the absence of external fields the equilibrium state of the fluid is characterized by an isotropic stress tensor. For small shear rates the stress tensor is characterized by the so called zero-shear material functions, namely, the static, zero-shear viscosity

$$
\eta:=\lim _{\dot{\gamma} \downarrow 0} \frac{\sigma_{x, y}}{\rho_{\mathrm{m}} \dot{\gamma}}
$$

and the first and second, static, zero-shear normal stress coefficients

$$
\Psi^{(1)}:=\lim _{\dot{\gamma} \downarrow 0} \frac{\sigma_{x, x}-\sigma_{y, y}}{\rho_{\mathrm{m}} \dot{\gamma}^{2}}, \quad \Psi^{(2)}:=\lim _{\dot{\gamma} \downarrow 0} \frac{\sigma_{y, y}-\sigma_{z, z}}{\rho_{\mathrm{m}} \dot{\gamma}^{2}} .
$$

Here $\rho_{\mathrm{m}}$ denotes the number density of monomers. For simplicity the quantities $(3.3)$ and (3.4) are simply referred to as the shear viscosity and the first and second normal stress coefficients hereafter.

\subsubsection{Linear viscoelasticity}

A straightforward approach to rheology of complex fluids can be given in the limit of small velocity gradients. Then the stress tensor (3.2) may be expanded in terms of the velocity gradient tensor and is given by a linear response relation. In the simple shear flow $u_{x}(t)=\dot{\gamma}(t) y$ this amounts to

$$
\sigma_{x, y}(t)=\int_{-\infty}^{t} \mathrm{~d} t^{\prime} G\left(t-t^{\prime}\right) \dot{\gamma}(t)
$$

for the shear stress which still includes the complete history of flow. The memory function $G(t)$ is referred to as the stress relaxation function. A characterization of the fluid by Eq. (3.5) pertains to the so called regime of linear viscoelasticity. Various phenomenological constitutive equations for polymeric liquids have been proposed, which mainly base on analogies with mechanical models. A comprehensive overview is given in ([Lar88]).

The equation (3.5) is now applied to predict the shear stress as a function of common experimental flow situations (see. Ch. 7.3 in [DoEd88]). In experiments the stresses are 
often measured as a function of the strains. By defining the shear strain $\gamma(t):=\int_{0}^{t} \mathrm{~d} t^{\prime} \dot{\gamma}\left(t^{\prime}\right)$ measured from the reference state $t=0$, the constitutive equation (3.5) can be integrated by parts

$$
\sigma_{x, y}=\int_{-\infty}^{t} \mathrm{~d} t^{\prime} \frac{\partial G\left(t-t^{\prime}\right)}{\partial t^{\prime}}\left(\gamma(t)-\gamma\left(t^{\prime}\right)\right)
$$

which relates the stress to the strain.

\subsubsection{Steady shear flow}

The simplest flow situation is a steady shear flow with a shear rate being constant in time $\dot{\gamma}(t)=\dot{\gamma}$. Then Eq. (3.5) predicts a constant shear stress

$$
\sigma_{x, y}=\dot{\gamma} \int_{0}^{\infty} \mathrm{d} t G(t)
$$

The shear viscosity (3.3) is then simply given by the integral over the stress relaxation function

$$
\eta=\frac{1}{\rho_{\mathrm{m}}} \int_{0}^{\infty} \mathrm{d} t G(t) .
$$

\subsubsection{Oscillatory shear}

Commonly small amplitude, oscillatory shear strains $\gamma(t)=\gamma_{0} \operatorname{Re}(\exp \{\mathrm{i} \omega t\})$ are applied to the sample in experiments. Here $\operatorname{Re}(\bullet)$ denotes the real part of a complex number. If the sinusoidal shear strain is inserted into Eq. (3.5) the stress

$$
\sigma_{x, y}(t)=\gamma_{0} \operatorname{Re}\left(\widehat{G}(\omega) \mathrm{e}^{\mathrm{i} \omega t}\right)
$$

is given in terms of the complex shear relaxation function which is defined by the Laplace transform

$$
\begin{aligned}
\widehat{G}(\omega): & =\mathrm{i} \omega \int_{0}^{\infty} \mathrm{d} t G(t) \mathrm{e}^{-\mathrm{i} \omega t} \\
& =: \widehat{G}^{\prime}(\omega)+\mathrm{i} \widehat{G}^{\prime \prime}(\omega) .
\end{aligned}
$$

The prefactor $\mathrm{i} \omega$ is commonly included in the definition of $\widehat{G}(\omega)$. The real and imaginary parts $\widehat{G}^{\prime}, \widehat{G}^{\prime \prime}$ denote the storage and loss modulus respectively which are the contribution of the stress which are in phase and out of phase with the strain, respectively. From the complex modulus the shear viscosity can be retrieved in the limit of small $\omega$. By comparing (3.8) with (3.10) one has

$$
\eta=\lim _{\omega \downarrow 0} \frac{1}{\rho_{\mathrm{m}}} \frac{\widehat{G}(\omega)}{\mathrm{i} \omega}
$$




\subsubsection{Creep flow}

In creep flow experiments a constant shear stress $\sigma_{x, y}=\sigma_{0}$ is applied at time $t=0$ and the shear strain $\gamma(t):=\int_{0}^{t} \mathrm{~d} t^{\prime} \dot{\gamma}\left(t^{\prime}\right)$ is measured as a function of time. The strain vanishes for $t<0$ whereas for $t>0$ it is determined by the integral equation [DoEd88]

$$
\sigma_{0}=\int_{0}^{t} \mathrm{~d} t^{\prime} G\left(t-t^{\prime}\right) \dot{\gamma}\left(t^{\prime}\right)
$$

which is easily solved by Laplace transformation in terms of $\widehat{G}(\omega)$ for large $t$. By neglecting contributions of order $\mathscr{O}\left(\omega^{3}\right)$ in $\widehat{G}(\omega)$ the Laplace inversion can be easily calculated and gives

$$
\gamma(t)=\sigma_{0}\left(\frac{t}{\eta}+J\right)
$$

for the asymptotic behavior of the shear strain for $t \rightarrow \infty$. Thereby, the zero-shear recoverable compliance

$$
J:=\frac{1}{\eta^{2}} \int_{0}^{\infty} \mathrm{d} t t G(t)
$$

is defined. Equation (3.14) nicely displays the main characteristics of a viscoelastic medium: For large but finite times it contains the constitutive equation $\gamma(t)=\sigma_{0} J$ of an elastic (Hookean) solid with a shear modulus $G=1 / J$, whereas for terminal times it recovers the constitutive equation $\dot{\gamma}(t)=\sigma_{0} / \eta$ of a viscous (Newtonian) fluid. In other words the recoverable compliance is the shear modulus of the material on large but finite time scales. This elastic behavior is transient, the material remains fluid-like unless the viscosity becomes infinite.

\subsubsection{Microscopic expression for stress tensor}

The main goal of molecular polymer dynamics is the derivation of a constitutive equation for the simple shear flow instead of assuming the linear viscoelastic model (3.5) with a phenomenological ansatz for $G(t)$. To this end a microscopic expression for the stress tensor in terms of the flow field is required. Here solely the excess stress due to the presence of the monomers is considered. The excess stress stems from the potential forces per unit area exerted by the monomers (for a sketch of the situation see Fig. 3.1) Following Ch. 3 in Ref. [DoEd88] or Ch. 16.3 in Ref. [BiCu87] the excess stress due to interacting monomers is given by

$$
\boldsymbol{\sigma}(t)=\left\langle-\frac{\rho_{\mathrm{m}}}{N} \sum_{i=1}^{N} \boldsymbol{F}_{i}(t) \boldsymbol{R}_{i}^{\dagger}(t)\right\rangle_{\mathrm{st}} .
$$

Here $\boldsymbol{F}_{i}(t):=-\partial U / \partial \boldsymbol{R}_{i}(t)$ is the force acting on monomer $i$ at time $t$. The average $\langle\bullet\rangle_{\text {st }}$, indicates an average over all monomer configurations in the steady state. Expression (3.16) is the shear stress in terms of the force per unit area, exerted by the monomers. It is simply 


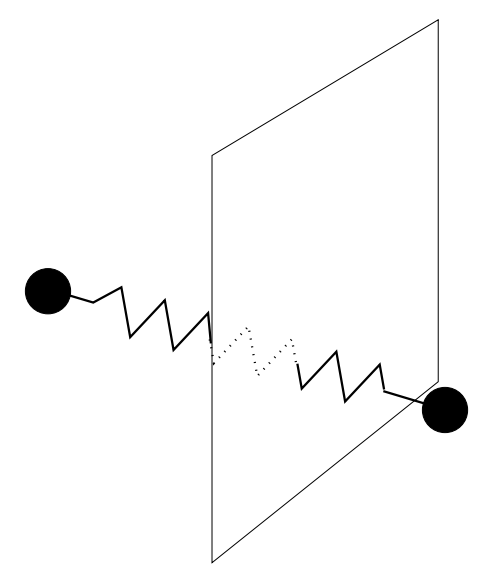

Figure 3.1: Additional stresses from the spring potentials

the potential part of the usual microscopic expression of the stress tensor for a collection of $N$ classical particles interacting via potential forces (see Ch. 2.5 in Ref. [BoYi91]).

Besides the excess contribution (3.16) from the monomers, there is also a solvent contribution. Commonly, it is assumed (see Ch. 3 in Ref. [DoEd88] or Ch. 16.3 in Ref. [BiCu87]) that the overall stress of the polymer-solvent mixture is simply given by the sum of (3.16) and a contribution of the Newtonian solvent in the absence of the polymers

$$
\boldsymbol{\sigma}_{\mathrm{s}}(t)=\eta_{\mathbf{s}}\left(\dot{\gamma}(t)+\dot{\gamma}^{\dagger}(t)\right)
$$

However, as pointed out in [OeRa89] this simple superposition of the stresses turns out to be inconsistent. The superposition is based on the assumption that the averaged solvent contribution to the stress is simply given by the gradient of the externally imposed velocity field $\dot{\gamma}$. This would imply that the contribution of the monomers to the velocity field $\boldsymbol{u}(\boldsymbol{r}, t)$ vanish when averaged over the monomer configurations. However, this is not correct. If the solvent contribution to the stress is computed from the gradient of the velocity field $\boldsymbol{u}(\boldsymbol{r}, t)$ by averaging over the monomer configurations an additional contribution arises which has been examined in [ScOt84].

\subsection{Density fluctuations}

\subsubsection{Dynamic light scattering}

Besides rheological properties which are probed by an external flow the critical behavior of a gelling system can also be revealed by microscopic density fluctuations in the absence of an external field. Microscopic density fluctuations can be measured directly by dynamic light or neutron scattering techniques.

In a solution of identical scattering units the electric field $\boldsymbol{E}_{\mathrm{i}}(t)$ of an incident light beam with polarization vector $\boldsymbol{n}_{\mathrm{i}}$ induces a dipole moment $\boldsymbol{\mu}(t)$ which depends on the 


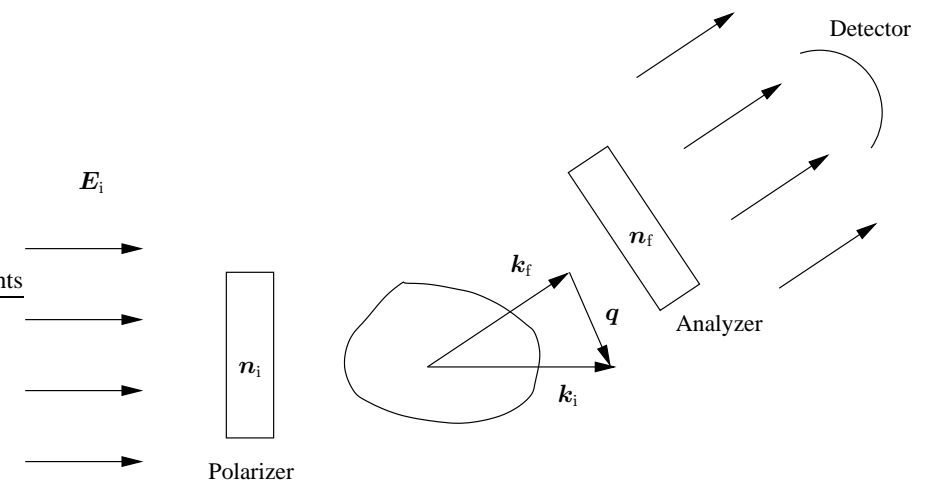

Figure 3.2: Scattering geometry

polarizability tensor $\boldsymbol{\alpha}$ of the scattering unit

$$
\boldsymbol{\mu}(t)=\boldsymbol{\alpha} \boldsymbol{E}(t) .
$$

As shown in [BePe76], the contribution of the electric field $\boldsymbol{E}_{\mathrm{f}}(\boldsymbol{q}, t)$ from monomer $j$ measured at detector at large distance is proportional to $\alpha_{\text {if }} \exp \left\{i \boldsymbol{q} \boldsymbol{R}_{j}(t)\right\}$, where

$$
\alpha_{\mathrm{if}}=\boldsymbol{n}_{\mathrm{f}} \cdot \boldsymbol{\alpha} \boldsymbol{n}_{\mathrm{i}}
$$

is characterized by the polarization vectors $\boldsymbol{n}_{\mathrm{i}}, \boldsymbol{n}_{\mathrm{f}}$ of the incoming and the scattered electric field, respectively. The scattering vector $\boldsymbol{q}=\boldsymbol{k}_{\mathrm{i}}-\boldsymbol{k}_{\mathrm{f}}$, or vector of momentum transfer, is defined by the difference of the wave vectors of the incoming and scattered field. If the scattering units are weakly coupled such that electronic states are only weakly perturbed by neighboring monomers the total electric field is proportional to the sum of independent contributions

$$
E_{\mathrm{f}}(\boldsymbol{q}, t) \propto \alpha_{\mathrm{if}} \sum_{j=1}^{N} \mathrm{e}^{\mathrm{i} \boldsymbol{q} \cdot \boldsymbol{R}_{j}(t)} .
$$

Equation (3.20) is of particular importance since it provides a direct relation between the electric field and fluctuations in the local density of monomers in the solution. This can be seen by considering the time-dependent, local number density of monomers per particle, as defined by

$$
\rho_{0}(\boldsymbol{r}, t):=\frac{1}{N} \sum_{i=1}^{N} \delta\left(\boldsymbol{r}-\boldsymbol{R}_{i}(t)\right) .
$$

It is convenient to write the density as the sum of the mean density per particle $V^{-1}$ and time-dependent fluctuations $\delta \rho_{0}(\boldsymbol{r}, t)$, viz $\rho_{0}(\boldsymbol{r}, t)=V^{-1}+\delta \rho_{0}(\boldsymbol{r}, t)$. The Fourier transform of the density is then given by

$$
\int_{V} \mathrm{~d}^{3} r \rho_{0}(\boldsymbol{r}, t) \mathrm{e}^{-\mathrm{i} \boldsymbol{q} \cdot \boldsymbol{r}}=\delta_{\boldsymbol{q}, 0}+\left(1-\delta_{\boldsymbol{q}, 0}\right) \delta \rho_{0}(\boldsymbol{q}, t)
$$


with

$$
\delta \rho_{0}(\boldsymbol{q}, t)=\frac{1}{N} \sum_{j=1}^{N} \mathrm{e}^{-\mathrm{i} \boldsymbol{q} \cdot \boldsymbol{R}_{j}(t)}
$$

Hence, the electric field $E_{\mathrm{f}}(\boldsymbol{q}, t)(3.20)$ at the detector with scattering vector $\boldsymbol{q} \neq 0$ is proportional to the spatial Fourier component $\delta \rho_{0}(\boldsymbol{q}, t)$ of fluctuations in the local density at wave vector $\boldsymbol{q}$.

\subsubsection{Coherent and incoherent scattering function}

The correlation functions which can be measured in a scattering experiment depend on the apparatus which processes the incoming electric field (3.20).

In the homodyne or self beating technique only the scattered light impinges on the photomultiplier which measures the intensity $I(\boldsymbol{q}, t)=E_{\mathrm{f}}(\boldsymbol{q}, t) E_{\mathrm{f}}^{*}(\boldsymbol{q}, t)$. If the signal is is passed into an autocorrelator subsequently, the intensity autocorrelation function or more precisely, its time average

$$
g_{I}(\boldsymbol{q}, t):=\left\langle I_{\mathrm{f}}(\boldsymbol{q}, 0) I_{\mathrm{f}}(\boldsymbol{q}, t)\right\rangle_{\mathrm{eq}} \sim N^{4}\left\langle|\delta \rho(\boldsymbol{q}, 0)|^{2}|\delta \rho(\boldsymbol{q}, t)|^{2}\right\rangle_{\mathrm{eq}}
$$

is measured. For ergodic systems the time average is equal to an average $\langle\bullet\rangle_{\text {eq }}$ over the equilibrium distribution of the macromolecules in solution.

In the heterodyne method the scattered electric field is mixed with a portion of unscattered laser light such that the photomultiplier measures the intensity of the sum of both signals. If the scattered and the unscattered electric fields are statistically independent and if the magnitude of the unscattered electric field is large when compared to the magnitude of the scattered field the heterodyne method provides access to the auto-correlation function of the electric field rather than its intensity by using a spectrum analyzer [BePe76, Ch. 4.3]. This procedure yields the correlation function

$$
g_{E}(\boldsymbol{q}, t):=\left\langle E_{\mathrm{f}}^{*}(\boldsymbol{q}, 0) E_{\mathrm{f}}(\boldsymbol{q}, t)\right\rangle_{\mathrm{eq}} \sim N^{2}\left\langle\delta \rho^{*}(\boldsymbol{q}, t) \delta \rho(\boldsymbol{q}, 0)\right\rangle_{\mathrm{eq}},
$$

where the asterisk denote complex conjugation. It is often convenient to define an intensive quantity, that is the coherent scattering function

$$
\begin{aligned}
S_{\mathrm{coh}}(\boldsymbol{q}, t): & =N^{-1} g_{E}(\boldsymbol{q}, t) \\
& =\frac{1}{N} \sum_{i, j=1}^{N}\left\langle\mathrm{e}^{\mathrm{i} \boldsymbol{q} \cdot\left[\boldsymbol{R}_{i}(t)-\boldsymbol{R}_{j}(0)\right]}\right\rangle_{\mathrm{eq}} .
\end{aligned}
$$

If the system is in equilibrium at $t=0$, then $S_{\mathrm{coh}}(\boldsymbol{q}, 0)$ is the static structure factor.

In general, the heterodyne and the homodyne correlation function measure different correlations. However, they can be related to each other in the special case if the electric field is a Gaussian random variable. This Gaussian approximation is valid if the scattering volume can be divided into a large number of sufficiently large subvolumes such that 
the motion of the subsystems can be regarded as independent. Then the electric field at the detector is a sum over independent contributions of the subregions, and according to the central limit theorem obeys Gaussian statistics. In experimental situations it is often stressed that this Gaussian approximation applies. For Gaussian statistics the four point correlation function $g_{I}(\boldsymbol{q}, t)$ can be written in terms of the two point correlation function $g_{E}(\boldsymbol{q}, t)$ according to (see App. 4.C in [BePe76])

$$
g_{I}(\boldsymbol{q}, t)=\left|g_{E}(\boldsymbol{q}, 0)\right|^{2}+\left|g_{E}(\boldsymbol{q}, t)\right|^{2}
$$

As pointed out by de Gennes [Gen79b] and Martin et al [MaWi91] it suffices to consider the self contributions from the coherent scattering function in (3.25). This defines the intermediate, incoherent scattering function

$$
S(\boldsymbol{q}, t):=\left\langle\frac{1}{N} \sum_{i=1}^{N} \mathrm{e}^{\mathrm{i} \boldsymbol{q} \cdot\left[\boldsymbol{R}_{i}(t)-\boldsymbol{R}_{i}(0)\right]}\right\rangle_{\mathrm{eq}} .
$$

However, the incoherent scattering function can be measured directly only in neutron scattering experiments.

\subsubsection{Cluster diffusion}

The simplest dynamical process which should contribute to density fluctuations at long times is the diffusion of clusters as a whole. The diffusion constant of a cluster is defined by the long time behavior of the mean squared displacement of the center of mass according to

$$
D:=\lim _{t \rightarrow \infty} \frac{1}{6 t}\left\langle\left[\boldsymbol{R}_{\mathrm{CM}}(t)-\boldsymbol{R}_{\mathrm{CM}}(0)\right]^{2}\right\rangle_{\mathrm{eq}} .
$$

Beside the diffusion constant of a single cluster it is convenient to define an effective diffusion constant for the gelling system by the time integral over the incoherent scattering function

$$
D_{\text {eff }}^{-1}:=\lim _{q \rightarrow 0} q^{2} \int_{0}^{\infty} \mathrm{d} t S(\boldsymbol{q}, t)
$$

which defines an effective time scale. For the special case of a single Brownian particle which is described by a Gaussian process the exponent in the incoherent scattering function (3.27) is basically given by the mean squared displacement (3.28) of the particle for long times. This yields an exponential decay $\exp \left\{-D q^{2} t\right\}$ of the scattering function and therefore the effective diffusion constant (3.29) coincides with (3.28).

\subsubsection{Nonlinear dynamic susceptibility}

In the long time limit for $t \rightarrow \infty$ the incoherent scattering function approaches its static limit. For long times the average $\langle\bullet\rangle_{\text {eq }}$ in $(3.27)$ is expected to factorize since the state at 
time $t$ is uncorrelated with that at time $t=0$. This implies

$$
\begin{aligned}
S_{\infty}(\boldsymbol{q}): & =\lim _{t \rightarrow \infty} S(\boldsymbol{q}, t) \\
& =\frac{1}{N} \sum_{j=1}^{N}\left\langle\mathrm{e}^{\mathrm{i} \boldsymbol{q} \cdot \boldsymbol{R}_{j}}\right\rangle_{\text {eq }}\left\langle\mathrm{e}^{-\mathrm{i} \boldsymbol{q} \cdot \boldsymbol{R}_{j}}\right\rangle_{\text {eq }} .
\end{aligned}
$$

In fact, $S_{\infty}(\boldsymbol{q})$ serves as an order parameter for the solidification transition to the amorphous solid state. This is in close analogy to the (temperature driven) spin glass transition in a system of spins $\sigma_{i}= \pm 1$ with random interactions: In the high temperature phase the system is paramagnetic. Below a critical temperature the ensemble averaged magnetization $1 / N \sum_{i=1}^{N}\left\langle\sigma_{i}\right\rangle_{\text {eq }}=0$ still lacks a preferred direction but in contrast the Edwards-Anderson order parameter $1 / N \sum_{i=1}^{N}\left\langle\sigma_{i}\right\rangle_{\text {eq }}\left\langle\sigma_{i}\right\rangle_{\text {eq }} \neq 0$ becomes nonzero, reflecting the subtle order of the spin glass phase.

For the gelation transition the situation is quite similar. Here, the transition cannot be revealed by equilibrium fluctuations of the density. The density remains constant throughout the transition and the particles localize around randomly and homogeneously distributed positions in space. Therefore, the Fourier transforme of the static density fluctuations

$$
\langle\delta \rho(\boldsymbol{q})\rangle_{\mathrm{eq}}=\frac{1}{N} \sum_{i=1}^{N}\left\langle\mathrm{e}^{\mathrm{i} \boldsymbol{q} \cdot \boldsymbol{R}_{j}}\right\rangle_{\mathrm{eq}}=0
$$

amounts to a sum of random phase factors and vanishes. In contrast the higher order density correlation $S_{\infty}(\boldsymbol{q})$ becomes nonzero.

The statistical mechanics approach to the vulcanization transition [GoCa96] admits to compute a simple but illustrative form of the order parameter in an exact mean field approach. Within this model the order parameter can be written as

$$
S_{\infty}(\boldsymbol{q})= \begin{cases}0, & c<c_{\text {crit }} \\ S_{\infty} \int_{0}^{\infty} \mathrm{d} x \pi(x) \mathrm{e}^{-(q \xi)^{2} / x}, & c>c_{\text {crit }}\end{cases}
$$

in the vicinity of the transition, where $q=|\boldsymbol{q}|$. The order parameter is zero in the solphase and acquires a nonzero value in the gel phase. It is determined by the fraction $S_{\infty} \sim\left(c-c_{\text {crit }}\right)$ of localized particles and by the distribution $\pi(x)$ of squares of inverse square localization lengths [CaGo94] which characterize the excursions of the localized particles around their mean positions. The length scale $\xi \sim\left|c-c_{\text {crit }}\right|^{-1 / 2}$ diverges when the transition is approached and plays the role of the correlation length.

Quite generally one would expect to observe precursors of the long range (connectivity) correlations in the liquid state when the transition is approached. Within the aforementioned model the built up of long ranged correlations is signalled by higher order correlations of density fluctuations, the divergence of the static nonlinear susceptibility

$$
\chi_{\infty}(\boldsymbol{q}):=\frac{1}{N} \sum_{i, j=1}^{N}\left\langle\mathrm{e}^{\mathrm{i} \boldsymbol{q} \cdot\left[\boldsymbol{R}_{i}-\boldsymbol{R}_{j}\right]}\right\rangle_{\mathrm{eq}}\left\langle\mathrm{e}^{-\mathrm{i} \boldsymbol{q} \cdot\left[\boldsymbol{R}_{i}-\boldsymbol{R}_{j}\right]}\right\rangle_{\mathrm{eq}}
$$


in the limit of $q \downarrow 0$.

In an analogous manner as the order parameter $S_{\infty}(\boldsymbol{q})$ is accessible via the long time decay of $S(\boldsymbol{q}, t)$, the static nonlinear susceptibility is the long time limit of a dynamic quantity $\chi_{\infty}(\boldsymbol{q}):=\lim _{t \rightarrow \infty} \chi(\boldsymbol{q}, t)$, the nonlinear dynamic susceptibility

$$
\chi(\boldsymbol{q}, t):=\left\langle\frac{1}{N} \sum_{i, j=1}^{N} \mathrm{e}^{\mathrm{i} \boldsymbol{q} \cdot\left[\boldsymbol{R}_{i}(t)-\boldsymbol{R}_{j}(t)\right]} \mathrm{e}^{-\mathrm{i} \boldsymbol{q} \cdot\left[\boldsymbol{R}_{i}(0)-\boldsymbol{R}_{j}(0)\right]}\right\rangle_{\mathrm{eq}} .
$$




\section{Chapter 4}

\section{Solution of the Rouse-Zimm model}

This chapter provides the exact solution of the equations of motion of the Rouse and the Zimm model where the generalization to random clusters requires some caution. Subsequently, the observables introduced in the previous chapter are computed from the solution of the Rouse-Zimm model.

\subsection{Solution of the equation of motion}

Due to the linearity of the equations of motion it is straightforward to obtain the solutions of the Langevin equations $(2.26,2.33)$ governing the Rouse and the Zimm model in simple shear flow [Rou53, Zim56],[DoEd88, Ch. 4],[BiCu87, Ch. 15]. In fact, the Rouse model is included in the Zimm model as the special case of vanishing hydrodynamic interaction parameter $\kappa=0$. Some observables like the diffusion constant are defined in the absence of external shear flow $\dot{\gamma}=0$ whereas the computation of the shear stress requires the presence of the flow, $\dot{\gamma} \neq 0$. Thus, obtaining the solution of the Zimm equation (2.33) in the presence of shear flow suffices to discuss all required cases by varying $\kappa$ and $\dot{\gamma}$. Nonetheless, before addressing the most general case $\kappa \neq 0, \dot{\gamma} \neq 0$, the special case $\kappa=0, \dot{\gamma}=0$ is examined which pertains to Rouse dynamics without shear flow. For this special case it is easier to demonstrate the relevance of the connectivity matrix $\Gamma$ for the dynamical evolution and to discuss the essential features of the solution.

\subsubsection{The simplest case: Rouse model without external fbw}

The Rouse equation in the absence of shear flow is formally an equation of motion for a system of coupled, overdamped, harmonic oscillators with the stochastic force as an inhomogeneity. Hence, it can be solved by standard methods. If the potential energy (2.15) is inserted into (2.26) the system of $N$ linear, first order, stochastic differential equations takes the familiar form

$$
\frac{\mathrm{d}}{\mathrm{d} t} \boldsymbol{R}_{i}(t)=-\frac{3 k_{\mathrm{B}} T}{\zeta a^{2}} \sum_{j=1}^{N} \Gamma_{i, j} \boldsymbol{R}_{j}(t)+\frac{1}{\zeta} \boldsymbol{\xi}_{i}(t),
$$


where $\boldsymbol{\xi}$ is characterized by $(2.27)$ and the initial data $\boldsymbol{R}\left(t_{0}\right)$ is fixed at time $t_{0}$. The solution of the stochastic equation (4.1) can formally be obtained, as if $\boldsymbol{\xi}_{i}(t)$ were a deterministic force [Arn92]

$$
\boldsymbol{R}_{i}(t)=\sum_{j=1}^{N}\left[\mathrm{e}^{-3 \Gamma\left(t-t_{0}\right) / t_{\mathrm{mic}}}\right]_{i, j} \boldsymbol{R}_{j}\left(t_{0}\right)+\frac{1}{\zeta} \int_{t_{0}}^{t} \mathrm{~d} t^{\prime}\left[\mathrm{e}^{-3 \Gamma\left(t-t^{\prime}\right) / t_{\mathrm{mic}}}\right]_{i, j} \boldsymbol{\xi}_{j}\left(t^{\prime}\right) .
$$

Here, the microscopic time scale

$$
t_{\text {mic }}:=\frac{\zeta a^{2}}{k_{\mathrm{B}} T}
$$

is defined in terms of the bare model parameters. In principle, the familiar procedure of transforming the solution (4.2) onto a basis of eigenvectors of the matrix $\Gamma$ is now applicable which would then yield the independent time evolution of the normal modes. The time scale associated with a normal mode is given by the corresponding inverse eigenvalue of $\Gamma$. Thus, the characteristic time scales in the Rouse model in units of $t_{\text {mic }}$ are determined by the eigenvalues of $\Gamma$.

The time evolution of the monomers' positions as given by the solution (4.2) is not deterministic. Due to the linear dependence on the Gaussian process $\boldsymbol{\xi}_{i}(t), \boldsymbol{R}_{i}(t)$ is itself a Gaussian process and thus completely characterized by its first two moments. If the initial condition $\boldsymbol{R}\left(t_{0}\right)$ is stochastically independent of $\boldsymbol{\xi}_{i}(t)$, the first moment can be computed from the solution (4.2) by employing the vanishing mean of $\boldsymbol{\xi}_{i}(t)$. This gives

$$
\overline{\boldsymbol{R}_{i}(t)}=\sum_{j=1}^{N}\left[\mathrm{e}^{-3 \Gamma\left(t-t_{0}\right) / t_{\mathrm{mic}}}\right]_{i, j} \boldsymbol{R}_{j}\left(t_{0}\right) .
$$

The second moment is computed by using the covariance (2.27), this yields

$$
\begin{aligned}
\overline{\boldsymbol{R}_{i}(t) \boldsymbol{R}_{j}^{\dagger}\left(t^{\prime}\right)} & =\sum_{m, n=1}^{N}\left[\mathrm{e}^{-3 \Gamma\left(t-t_{0}\right) / t_{\mathrm{mic}}}\right]_{i, m} \boldsymbol{R}_{m}\left(t_{0}\right) \boldsymbol{R}_{n}^{\dagger}\left(t_{0}\right)\left[\mathrm{e}^{-3 \Gamma\left(t-t_{0}\right) / t_{\mathrm{mic}}}\right]_{n, j} \\
& +\frac{2 k_{\mathrm{B}} T}{\zeta} \int_{t_{0}}^{\min \left(t, t^{\prime}\right)} \mathrm{d} t^{\prime \prime}\left[\mathrm{e}^{-3 \Gamma\left(t-t^{\prime}-2 t^{\prime \prime}\right) / t_{\mathrm{mic}}}\right]_{i, j} \mathbf{1} .
\end{aligned}
$$

The Langevin equation (4.1) is designated to describe dynamics of the macromolecules in equilibrium if the initial condition is fixed in the infinite past $t_{0} \rightarrow-\infty$ and transient behavior has died out. However, one cannot expect that equilibrium characteristics can be obtained by simply taking the limit $t_{0} \rightarrow-\infty$ of the first and second moment (4.4) and (4.5). This is due to the fact that the vector $\boldsymbol{R}_{i}(t)$ describes the absolute position of monomer $i$ which is influenced by the motion of the center of mass of the cluster $\mathscr{N}(i)$ which $i$ belongs to

$$
\boldsymbol{R}_{\mathrm{cm}}(\mathscr{N}(i)):=\frac{1}{N(i)} \sum_{j \in \mathscr{N}(i)} \boldsymbol{R}_{j}
$$


The position of the center of mass is a degree of freedom which cannot be equilibrated since it diffuses freely. This is easily seen by summing the Rouse equation (4.1) over all $j \in \mathscr{N}(i)$ and dividing by the number $N(i)$ of monomers in that cluster. This yields a simple diffusion equation for the center of mass

$$
\frac{\mathrm{d}}{\mathrm{d} t} \boldsymbol{R}_{\mathrm{cm}}(t \mid \mathscr{N}(i))=\frac{1}{\zeta N(i)} \sum_{j \in \mathscr{N}(i)} \boldsymbol{\xi}_{j}(t)
$$

with a diffusion constant which is inversely proportional to the size of the cluster. Thus, only position vectors relative to the centers of mass are expected to reach equilibrium for $t_{0} \rightarrow-\infty$. In order to see how this is encoded in the first and second moment (4.4) and (4.5) one observes that in the limit $t_{0} \rightarrow-\infty$,

$$
\lim _{t_{0} \rightarrow-\infty} \mathrm{e}^{-3 \Gamma\left(t-t_{0}\right) / t_{\mathrm{mic}}}=E_{0}
$$

only the projector $E_{0}$ onto the nullspace of $\Gamma$ survives. A fact which is mathematically easily understood by means of the spectral decomposition of the matrix $\Gamma$ as shown in App. B.7. Equation (4.8) implies that equilibrium can only be reached for coordinates in the subspace $\left(\mathbb{1}-E_{0}\right) \mathbb{R}^{N} \times \mathbb{R}^{3}$ which is spanned by position vectors relative to the centers of mass. To see this explicitly, the position vector of monomer $i$ relative to the center of mass of its cluster $\mathscr{N}(i)$ is expressed in terms of $\left(\mathbb{1}-E_{0}\right)$ via

$$
\boldsymbol{R}_{i}-\boldsymbol{R}_{\mathrm{cm}}(\mathscr{N}(i))=\boldsymbol{R}_{i}-\frac{1}{N(i)} \sum_{j \in \mathscr{N}(i)} \boldsymbol{R}_{j}=\sum_{j=1}^{N}\left[\mathbb{1}-E_{0}(\mathscr{G})\right]_{i, j} \boldsymbol{R}_{j}
$$

which follows (see also Eq. (2.21)).

For observables which reach equilibrium for $t_{0} \rightarrow-\infty$, additionally the limit $t \rightarrow \infty$ can be considered. This corresponds to the static limit. Averages for $t \rightarrow \infty$ coincide with averages over the Boltzmann distribution. For instance the mean squared distance $\left(\boldsymbol{R}_{i}-\boldsymbol{R}_{j}\right)^{2}$ between two monomers $i$ and $j$ belonging to the same cluster can be computed in either way

$$
\lim _{t \rightarrow \infty} \lim _{t \rightarrow \infty} \overline{\left(\boldsymbol{R}_{i}(t)-\boldsymbol{R}_{j}(t)\right)^{2}}=\left\langle\left(\boldsymbol{R}_{i}-\boldsymbol{R}_{j}\right)^{2}\right\rangle_{\text {eq }}=a^{2} \mathscr{R}_{i, j}
$$

\subsubsection{Rouse-Zimm model with shear fbw}

Next, the most general case of the Rouse-Zimm equations (2.33) is considered, that is nonvanishing hydrodynamic interactions $\kappa \neq 0$ and nonvanishing shear rate $\dot{\gamma} \neq 0$. To this end the initial condition $\boldsymbol{R}\left(t_{0}\right)$ is again fixed at time $t_{0}$ and the coordinate transformation

$$
\boldsymbol{R}_{i}(t)=: \sum_{j=1}^{N}\left[H^{\frac{1}{2}}\right]_{i, j} \boldsymbol{X}_{j}(t)
$$


is applied to the Zimm equation (2.33). This yields

$$
\zeta\left(\frac{\mathrm{d}}{\mathrm{d} t} \boldsymbol{X}_{i}(t)-\dot{\gamma}(t) \boldsymbol{X}_{i}\right)=-\sum_{j=1}^{N} \widetilde{\Gamma}_{i, j} \boldsymbol{X}_{j}(t)+\boldsymbol{\xi}_{i}(t)
$$

where the transformed white noise $\boldsymbol{\xi}_{i}(t)$ is characterized by zero mean and co-variance

$$
\overline{\boldsymbol{\xi}_{i}(t) \boldsymbol{\xi}_{j}^{\dagger}\left(t^{\prime}\right)}=2 k_{\mathrm{B}} T \zeta \delta_{i, j} \delta\left(t-t^{\prime}\right) \mathbf{1}
$$

In writing down (4.12) a generalized connectivity matrix

$$
\widetilde{\Gamma}:=H^{\frac{1}{2}} \Gamma H^{\frac{1}{2}}
$$

has been defined. The resulting equation (4.12) of motion for the coordinates $\boldsymbol{X}_{i}$ coincides formally with the equation in the Rouse model (2.26) for crosslinked monomers [BrGo97, BrLo99, BrLo01a], but with a formal connectivity matrix $\widetilde{\Gamma}$ which plays the analogous role for the Zimm model as $\Gamma$ does for the Rouse model: The inverse eigenvalues of $\widetilde{\Gamma}$ determine the relaxation times in the Zimm model. The matrix $\widetilde{\Gamma}$ is a positive semidefinite matrix and the dimension of the null space is given by the number of clusters. The projector on the subspace of zero eigenvalues of $\widetilde{\Gamma}$ is denoted by $\widetilde{E}_{0}$ and an explicit form can be given in terms of the projector $E_{0}$ of $\Gamma$

$$
\widetilde{E}_{0}=\frac{H^{-\frac{1}{2}} E_{0} H^{-\frac{1}{2}}}{\operatorname{Tr}\left(H^{-1} E_{0}\right)}
$$

Here $\operatorname{Tr}(\bullet)$ denotes the trace of a $N \times N$ matrix. The spectral properties of $\widetilde{\Gamma}$ are characterized in App. B.7.

When Zimm dynamics is addressed in the literature, it is common to decouple Eq. (2.33) by a transformation to normal coordinates of the nonsymmetric matrix $H \Gamma$. Here, the transformation involving the symmetric matrix $\widetilde{\Gamma}$ is preferred since spectral decompositions of symmetric matrices are easier to handle. Indeed, both transformations are equivalent and the relations amongst their spectral properties are shown in App. B.2.

The solution of the the linear stochastic differential equation (4.12) is obtained by standard methods (see, e.g. Ch. 8 in [Arn92]). The solution for transformed initial data $\boldsymbol{X}_{i}\left(t_{0}\right)=\sum_{j=1}^{N}\left[H^{-\frac{1}{2}}\right]_{i, j} \boldsymbol{R}_{j}\left(t_{0}\right)$ is given by (cf. Section II.C. in [BrLo01a])

$$
\boldsymbol{X}_{i}(t)=\sum_{j=1}^{N} U_{i, j}\left(t-t_{0}\right) \boldsymbol{T}\left(t, t_{0}\right) \boldsymbol{X}_{j}\left(t_{0}\right)+\frac{1}{\zeta} \int_{t_{0}}^{t} \mathrm{~d} t^{\prime} U_{i, j}\left(t-t^{\prime}\right) \boldsymbol{T}\left(t, t^{\prime}\right) \boldsymbol{\xi}_{j}\left(t^{\prime}\right) .
$$

The time evolution is now characterized by two time quantities. First, the time evolution generated by the potential force is characterized by the matrix elements $U_{i, j}(t):=[U(t)]_{i, j}$ of the exponential of the generalized connectivity matrix

$$
U(t):=\mathrm{e}^{-3 \widetilde{\Gamma} t / t_{\mathrm{mic}}}
$$


with the microscopic time scale $t_{\text {mic }}$ being defined in (4.3).

Second, the $3 \times 3$ tensor $\boldsymbol{T}\left(t, t^{\prime}\right)$ characterizes the time evolution the external flow field. The validity of (4.19) requires $\partial_{t} \boldsymbol{T}\left(t, t^{\prime}\right)=\dot{\gamma}(t) \boldsymbol{T}\left(t, t^{\prime}\right)$ with initial condition $\boldsymbol{T}(t, t)=\mathbf{1}$. For an arbitrary velocity gradient tensor the solution $\boldsymbol{T}\left(t, t^{\prime}\right)$ is given by the time-ordered exponential of the integral over $\dot{\gamma}(\tau)$. However, for the simple shear flow (2.6) one has $\dot{\gamma}(t) \dot{\gamma}\left(t^{\prime}\right)=0$ for all $t, t^{\prime}$ and therefore only the first two terms are nonzero

$$
\boldsymbol{T}\left(t, t^{\prime}\right):=\mathbf{1}+\int_{t^{\prime}}^{t} \mathrm{~d} t^{\prime \prime} \dot{\gamma}\left(t^{\prime \prime}\right)
$$

The solution $\boldsymbol{R}_{i}(t)$ of the Zimm equation (2.33) is obtained by inserting (4.16) in (4.11)

$$
\begin{aligned}
\boldsymbol{R}_{i}(t)= & \sum_{j=1}^{N}\left[H^{\frac{1}{2}} U\left(t-t_{0}\right) H^{-\frac{1}{2}}\right]_{i, j} \boldsymbol{T}\left(t, t_{0}\right) \boldsymbol{R}_{j}\left(t_{0}\right) \\
& +\frac{1}{\zeta} \int_{t_{0}}^{t} \mathrm{~d} t^{\prime}\left[H^{\frac{1}{2}} U\left(t-t^{\prime}\right)\right]_{i, j} \boldsymbol{T}\left(t, t^{\prime}\right) \boldsymbol{\xi}_{j}\left(t^{\prime}\right) .
\end{aligned}
$$

Since $\boldsymbol{\xi}_{i}(t)$ is a Gaussian process, so is $\boldsymbol{R}_{i}(t)$ due to the linearity of the equation of motion. Hence, it is again completely characterized by its first two moments. If the initial condition $\boldsymbol{R}_{j}\left(t_{0}\right)$ is stochastically independent of $\boldsymbol{\xi}_{i}(t)$ the mean value is given by

$$
\overline{\boldsymbol{R}_{i}(t)}=\sum_{j=1}^{N}\left[H^{\frac{1}{2}} U\left(t-t_{0}\right) H^{-\frac{1}{2}}\right]_{i, j} \boldsymbol{T}\left(t, t_{0}\right) \boldsymbol{R}_{j}\left(t_{0}\right)
$$

and does not vanish in general. The matrix of second moments of $\boldsymbol{R}_{i}(t)$ is given by

$$
\begin{aligned}
\overline{\boldsymbol{R}_{i}(t) \boldsymbol{R}_{j}^{\dagger}\left(t^{\prime}\right)} & =\sum_{m, n=1}^{N}\left\{\left[H^{\frac{1}{2}} U\left(t-t_{0}\right) H^{-\frac{1}{2}}\right]_{i, m}\right. \\
& \left.\boldsymbol{T}\left(t, t_{0}\right) \boldsymbol{R}_{m}\left(t_{0}\right) \boldsymbol{R}_{n}\left(t_{0}\right)^{\dagger} \boldsymbol{T}^{\dagger}\left(t^{\prime}, t_{0}\right)\left[H^{-\frac{1}{2}} U\left(t^{\prime}-t_{0}\right) H^{\frac{1}{2}}\right]_{n, j}\right\} \\
+ & \frac{2 k_{\mathrm{B}} T}{\zeta} \int_{t_{0}}^{\min \left(t, t^{\prime}\right)} \mathrm{d} t^{\prime \prime}\left[H^{\frac{1}{2}} U\left(t+t^{\prime}-2 t^{\prime \prime}\right) H^{\frac{1}{2}}\right]_{i, j} \boldsymbol{T}\left(t, t^{\prime \prime}\right) \boldsymbol{T}^{\dagger}\left(t^{\prime}, t^{\prime \prime}\right) .
\end{aligned}
$$

As demonstrated in the previous section, in the absence of shear flow equilibrium dynamics is reached as the stationary state on the subspace $\left(\mathbb{1}-E_{0}\right) \mathbb{R}^{N} \times \mathbb{R}^{3}$ for $t \rightarrow-\infty$. Here, in contrast, a non-equilibrium, stationary, state is reached on that subspace due to the presence of the shear flow acting as a driving force. By taking the limit $t_{0} \rightarrow-\infty$ of the exponential $U(t)$ only the projector $\widetilde{E}_{0}$ onto the subspace of zero eigenvalues $\widetilde{\Gamma}$ survives

$$
\lim _{t_{0} \rightarrow-\infty} U\left(t-t_{0}\right)=\widetilde{E}_{0}=\frac{H^{-\frac{1}{2}} E_{0} H^{-\frac{1}{2}}}{\operatorname{Tr}\left(E_{0} H^{-1}\right)} .
$$


Here the representation (4.15) is employed for $\widetilde{E}_{0}$. By virtue of Eq. (4.22) the limit $t_{0} \rightarrow$ $-\infty$ of the moments $(4.20)$ and (4.21) is meaningful only on the subspace $\left(\mathbb{1}-E_{0}\right) \mathbb{R}^{N} \times \mathbb{R}^{3}$ where one has a vanishing mean and the steady state co-variance

$$
\begin{aligned}
& \boldsymbol{C}_{i, j}\left(t, t^{\prime}\right):=\lim _{t_{0} \rightarrow-\infty} \sum_{m, n=1}^{N}\left[\mathbb{1}-E_{0}\right]_{i, m} \overline{\boldsymbol{R}_{m}(t) \boldsymbol{R}_{n}^{\dagger}\left(t^{\prime}\right)}\left[\mathbb{1}-E_{0}\right]_{m, j} \\
&=\frac{2 k_{\mathrm{B}} T}{\zeta} \int_{-\infty}^{\min \left(t, t^{\prime}\right)} \mathrm{d} t^{\prime \prime}\left\{\left[\left(\mathbb{1}-E_{0}\right) H^{\frac{1}{2}} U\left(t+t^{\prime}-2 t^{\prime \prime}\right) H^{\frac{1}{2}}\left(\mathbb{1}-E_{0}\right)\right]_{i, j}\right. \\
&\left.\boldsymbol{T}\left(t, t^{\prime \prime}\right) \boldsymbol{T}^{\dagger}\left(t^{\prime}, t^{\prime \prime}\right) .\right\}
\end{aligned}
$$

The co-variance is a function of $t$ and $t^{\prime}$ if the shear rate depends on time. For a constant shear rate $\dot{\gamma}=$ const the strain tensor $\boldsymbol{T}\left(t, t^{\prime}\right)=\mathbf{1}+\left(t-t^{\prime}\right) \dot{\gamma} \equiv \boldsymbol{T}\left(t-t^{\prime}\right)$ depends only on the difference $t-t^{\prime}$. In this case $\boldsymbol{R}_{i}(t)$ is a stationary Gaussian process on this subspace.

Expectation values of observables in the stationary state can be computed for $t_{0} \rightarrow-\infty$ from the solution of the Rouse-Zimm equation.

\subsubsection{Rouse-Zimm model without external fbw}

Finally, Rouse-Zimm dynamics is considered in the presence of hydrodynamic interactions $\kappa \neq 0$ and in the absence of the external driving force $\dot{\gamma}=0$. It is necessary to examine this case separately, since for diffusion constants and the incoherent scattering function the increment or displacement $\left(\boldsymbol{R}_{i}(t)-\boldsymbol{R}_{i}(0)\right)$ of monomer $i$ is of special interest which is well-defined only in the absence of the shear flow.

The displacement is immediately obtained from the solution (4.19) by observing $\boldsymbol{T}\left(t, t^{\prime}\right)=\mathbf{1}$ in the absence of shear flow $\dot{\gamma}=0$. This gives rise to

$$
\begin{aligned}
\boldsymbol{R}_{i}(t)-\boldsymbol{R}_{i}(0)=\sum_{j=1}^{N} & {\left[H^{\frac{1}{2}} U\left(-t_{0}\right)(U(t)-\mathbb{1}) H^{-\frac{1}{2}}\right]_{i, j} \boldsymbol{R}_{j}\left(t_{0}\right) } \\
& +\frac{1}{\zeta} \int_{t_{0}}^{t} \mathrm{~d} t^{\prime}\left[H^{\frac{1}{2}} U\left(t-t^{\prime}\right)\right]_{i, j} \boldsymbol{\xi}_{j}\left(t^{\prime}\right) \\
& -\frac{1}{\zeta} \int_{t_{0}}^{0} \mathrm{~d} t^{\prime}\left[H^{\frac{1}{2}} U\left(-t^{\prime}\right)\right]_{i, j} \boldsymbol{\xi}_{j}\left(t^{\prime}\right) .
\end{aligned}
$$

The displacement is a linear combination of Gaussian processes and thus is itself Gaussian. For $t_{0} \rightarrow-\infty$ the mean value of the displacement vanishes

$$
\lim _{t_{0} \rightarrow-\infty} \overline{\left(\boldsymbol{R}_{i}(t)-\boldsymbol{R}_{i}(0)\right)}=0
$$

which follows from the identity $(U(t)-\mathbb{1})=\left(\mathbb{1}-\widetilde{E}_{0}\right)(U(t)-\mathbb{1})$ and the application of (4.22) in the limit $t_{0} \rightarrow \infty$. 
The second moment in equilibrium is obtained after a little algebra (see App. C). It can be written in the form

$$
\begin{aligned}
\boldsymbol{D}_{i, j}(t): & =\lim _{t_{0} \rightarrow-\infty} \overline{\left(\boldsymbol{R}_{i}(t)-\boldsymbol{R}_{i}(0)\right)\left(\boldsymbol{R}_{j}(t)-\boldsymbol{R}_{j}(0)\right)^{\dagger}} \\
& =\frac{2 k_{\mathrm{B}} T}{\zeta} \int_{0}^{t} \mathrm{~d} t^{\prime}\left[H^{\frac{1}{2}} U\left(t^{\prime}\right) H^{\frac{1}{2}}\right]_{i, j} \mathbf{1} \\
& =: D_{i, j}(t) \mathbf{1} .
\end{aligned}
$$

\subsubsection{Consistency of the Zimm model}

One may ask if the preaveraging in the Zimm model is a consistent approximation in the following sense: Zimm dynamics with a mobility matrix $H$, which is preaveraged with the Boltzmann distribution, should recover the Boltzmann distribution afterwards in the static limit $t \rightarrow \infty$ in order to guarantee

$$
\lim _{t \rightarrow \infty} \lim _{t_{0} \rightarrow-\infty} \overline{\boldsymbol{H}_{i, j}\left(\boldsymbol{R}_{i}(t)-\boldsymbol{R}_{j}(t)\right)}=\left\langle\boldsymbol{H}_{i, j}\left(\boldsymbol{R}_{i}-\boldsymbol{R}_{j}\right)\right\rangle_{\mathrm{eq}}=H_{i, j} \mathbf{1}
$$

This is indeed the case, which can be seen by considering the second moment (4.23) in the absence of shear flow, i.e. $\boldsymbol{T}\left(t, t^{\prime}\right)=1$. In App. B.3 it is then proved that

$$
\left.\lim _{t \rightarrow \infty} \boldsymbol{C}_{i, j}(t, t)\right|_{\dot{\gamma}=0}=\frac{a^{2}}{3}\left[\frac{1-E_{0}}{\Gamma}\right]_{i, j} \mathbf{1}
$$

is valid independent of the preaveraged mobility matrix $H$. This implies that in the absence of shear flow Rouse- and Zimm dynamics approach identical equilibrium states for $t \rightarrow \infty$ on the subspace $\left(\mathbb{1}-E_{0}\right) \mathbb{R}^{N} \times \mathbb{R}^{3}$. Within this subspace equilibrium averages can be computed either with the Boltzmann weight $\exp \left\{-U(\{\boldsymbol{R}\}) / k_{\mathrm{B}} T\right\}$ or by taking the limit $t_{0} \rightarrow-\infty, t \rightarrow \infty$ of the average over the thermal noise. For instance, the formula (4.10) for the mean squared distance between two monomers on the same cluster is valid for Rouse and for Zimm dynamics which follows directly from (4.30). In the same way the validity of (4.29) can be shown.

\subsection{Viscoelasticity in the Rouse-Zimm model}

\subsubsection{Stress tensor}

For the computation of the stress tensor (3.16) within the Rouse-Zimm model in the stationary state the average $\langle\bullet\rangle_{\text {st }}$ is computed by fixing the initial condition $\boldsymbol{R}_{i}\left(t_{0}\right)$ in the infinite past $t_{0} \rightarrow-\infty$. By inserting the force $\boldsymbol{F}_{i}(t)=-3 k_{\mathrm{B}} T / a^{2} \sum_{j=1}^{N} \Gamma_{i, j} \boldsymbol{R}_{j}(t)$ on monomer $i$, the stress (3.16) is expressed in terms of the second moment $\boldsymbol{C}_{i, j}(t, t)$ from 
Eq. (4.23) via

$$
\begin{aligned}
\boldsymbol{\sigma}(t \mid \mathscr{G}) & =\lim _{t_{0} \rightarrow-\infty}-\frac{\rho_{\mathrm{m}}}{N} \sum_{i=1}^{N} \overline{\boldsymbol{F}_{i}(t) \boldsymbol{R}_{i}^{\dagger}(t)} \\
& =\frac{3 k_{\mathrm{B}} T \rho_{\mathrm{m}}}{t_{\text {mic }} N} \sum_{i, j=1}^{N} \Gamma_{i, j} \boldsymbol{C}_{i, j}(t, t) \\
& =\frac{6 k_{\mathrm{B}} T \rho_{\mathrm{m}}}{t_{\text {mic }} N} \int_{-\infty}^{t} \mathrm{~d} t^{\prime} \operatorname{Tr}\left[\widetilde{\Gamma} U\left(2\left(t-t^{\prime}\right)\right)\right] \boldsymbol{B}\left(t, t^{\prime}\right) .
\end{aligned}
$$

Here, $\operatorname{Tr}(\bullet)$ denotes the trace of an $N \times N$ matrix, $\boldsymbol{B}\left(t, t^{\prime}\right):=\boldsymbol{T}\left(t, t^{\prime}\right) \boldsymbol{T}^{\dagger}\left(t, t^{\prime}\right)$ is referred to as the Finger strain tensor and the last step in (4.31) employs the cyclic invariance of the trace and the definition of the generalized connectivity matrix $\widetilde{\Gamma}(4.14)$. The dependence on the crosslink realization $\mathscr{G}$ is made explicit.

Now, the shear stress relaxation function is introduced

$$
G(t \mid \mathscr{G}):=\frac{k_{\mathrm{B}} T \rho_{\mathrm{m}}}{N} \operatorname{Tr}\left(\left(1-\widetilde{E}_{0}(\mathscr{G})\right) \exp \left\{-\frac{6 t}{t_{\mathrm{mic}}} \widetilde{\Gamma}(\mathscr{G})\right\}\right)
$$

in terms of which the monomer contribution to the stress can be written as

$$
\boldsymbol{\sigma}(t)=\int_{-\infty}^{t} \mathrm{~d} t^{\prime}\left(\frac{\partial}{\partial t^{\prime}} G\left(t-t^{\prime}\right)\right) \boldsymbol{B}\left(t, t^{\prime}\right)
$$

Integrating by parts and writing down the matrix explicitly yields

$$
\boldsymbol{\sigma}(t \mid \mathscr{G})=G(0) \mathbf{1}+\int_{-\infty}^{t} \mathrm{~d} t^{\prime} G\left(t-t^{\prime} \mid \mathscr{G}\right) \dot{\gamma}\left(t^{\prime}\right)\left(\begin{array}{ccc}
2 \int_{t^{\prime}}^{t} \mathrm{~d} s \dot{\gamma}(s) & 1 & 0 \\
1 & 0 & 0 \\
0 & 0 & 0
\end{array}\right)
$$

for the stress tensor in the Rouse-Zimm model. It is determined by a single function, the shear relaxation function which is a sum of $N-K$ exponentials. The exponentials describe the relaxations of the normal modes of $\widetilde{\Gamma}$ which are associated with nonvanishing eigenvalues. Hence, the eigenvalues determine the memory of the stress tensor, i.e. how the stress at time $t$ is influenced by the shear flow at time $t^{\prime}<t$.

The excess shear stress $\sigma_{x, y}$ from the polymers is given by a linear response relation which is valid for arbitrary shear rates $\dot{\gamma}(t)$. In other words the Rouse-Zimm model is a linear viscoelastic model for the shear stress (cf. Eq. (3.5)). The fact that shear stress depends linearly on the shear rate justifies the use of the equations for the material functions in Sec. 3.1.2 which were derived under the assumption of linear viscoelasticity. The viscosity and the recoverable compliance can be computed from the formulas (3.8) and (3.15), respectively, by using the shear relaxation function (4.32). Note, that the shear relaxation function $G(t)$ can be also computed from the stress autocorrelation function in the absence of shear flow. Then, the shear viscosity (3.8) amounts to a Green-Kubo formula (see Ch. 2.5 in [BoYi91]). Besides the linear response for the shear stress, (4.34) predicts non-isotropic normal stresses. This result is beyond linear viscoelasticity. 


\subsubsection{Stress relaxation function}

Due to the independent motion of the clusters which is reflected by the block diagonal form of $\widetilde{\Gamma}$ the stress relaxation function $G(t)(4.32)$ can be decomposed into contributions from different clusters according to

$$
G(t \mid \mathscr{G})=\sum_{k=1}^{K} \frac{N_{k}}{N} G\left(t \mid \mathscr{N}_{k}\right)
$$

The contribution from cluster $N_{k}$ is given by

$$
G\left(t \mid \mathscr{N}_{k}\right):=\frac{k_{\mathrm{B}} T \rho_{\mathrm{m}}}{N_{k}} \operatorname{Tr}\left(\left(1-\widetilde{E}_{0}\left(\mathscr{N}_{k}\right)\right) \exp \left\{-\frac{6 k_{\mathrm{B}} T}{a^{2}} \widetilde{\Gamma}\left(\mathscr{N}_{k}\right) t\right\}\right) .
$$

\subsubsection{Shear viscosity}

For a constant shear rate $\dot{\gamma}(t)=\dot{\gamma}$ the viscosity is given by (3.8). The integral is readily carried out giving

$$
\begin{aligned}
\eta(\mathscr{G}) & =\frac{1}{\rho_{\mathrm{m}}} \int_{0}^{\infty} \mathrm{d} t^{\prime} G\left(t^{\prime} \mid \mathscr{G}\right) \\
& =\frac{a^{2}}{3} \frac{1}{2 N} \operatorname{Tr}\left(\frac{1-\widetilde{E}_{0}(\mathscr{G})}{\widetilde{\Gamma}(\mathscr{G})}\right)
\end{aligned}
$$

Thus, the viscosity is given by the trace of the Moore Penrose inverse, that is the sum over the nonzero eigenvalues of the generalized connectivity matrix $\widetilde{\Gamma}$. The dependence on the crosslink configuration $\mathscr{G}$ in Eq. (4.36) is made explicit.

According to the cluster decomposition of $G(t \mid \mathscr{G})$ in $(4.36)$ the viscosity is also decomposed into additive contributions from individual clusters $\mathscr{N}_{k}$ according to

$$
\eta(\mathscr{G})=\sum_{k=1}^{K} \frac{N_{k}}{N} \eta\left(\mathscr{N}_{k}\right)
$$

where the contribution of the cluster $\mathscr{N}_{k}$ is defined by

$$
\eta\left(\mathscr{N}_{k}\right):=\frac{a^{2}}{3} \frac{1}{2 N_{k}} \operatorname{Tr}\left(\frac{1-\widetilde{E}_{0}\left(\mathscr{N}_{k}\right)}{\widetilde{\Gamma}\left(\mathscr{N}_{k}\right)}\right)
$$

\subsubsection{Normal stresses}

In view of the stress tensor (4.34), the second normal stress coefficient $\Psi^{(2)}$ defined in Eq. (3.4) vanishes for arbitrary shear rate $\dot{\gamma}(t)$. This result holds for both, the Rouse and the Zimm model. 
In contrast, for time independent shear rate $\dot{\gamma}(t)=\dot{\gamma}$ the first normal stress coefficient defined in Eq. (3.4) is nonzero and given by

$$
\Psi^{(1)}(\mathscr{G})=\frac{2}{\rho_{\mathrm{m}}} \int_{0}^{\infty} \mathrm{d} t t G(t \mid \mathscr{G})
$$

The integration is carried out and $\Psi^{(1)}$ is expressed in terms of the generalized connectivity matrix via

$$
\Psi^{(1)}(\mathscr{G})=\frac{1}{k_{\mathrm{B}} T}\left(\frac{a^{2}}{3}\right)^{2} \frac{1}{2 N} \operatorname{Tr}\left[\frac{1-\widetilde{E}_{0}(\mathscr{G})}{(\widetilde{\Gamma}(\mathscr{G}))^{2}}\right] .
$$

Again, the cluster decomposition of $G(t)$ implies the decomposition of $\Psi^{(1)}$ into contributions from individual clusters $\mathscr{N}_{k}$

$$
\Psi^{(1)}(\mathscr{G})=\sum_{k=1}^{K} \frac{N_{k}}{N} \Psi^{(1)}\left(\mathscr{N}_{k}\right)
$$

with

$$
\Psi^{(1)}\left(\mathscr{N}_{k}\right)=\frac{1}{k_{\mathrm{B}} T}\left(\frac{a^{2}}{3}\right)^{2} \frac{1}{2 N_{k}} \operatorname{Tr}\left[\frac{1-\widetilde{E}_{0}\left(\mathscr{N}_{k}\right)}{\left(\widetilde{\Gamma}\left(\mathscr{N}_{k}\right)\right)^{2}}\right]
$$

\subsubsection{Recoverable compliance}

The recoverable compliance defined by Eq. (3.15) can be computed from the viscosity (4.36) and the first normal stress coefficient (4.39) according to

$$
J(\mathscr{G})=\frac{\Psi^{(1)}(\mathscr{G})}{2 \eta(\mathscr{G})^{2}}
$$

\subsection{Density fluctuations within the Rouse-Zimm model}

\subsubsection{Diffusion constants}

Note, that density fluctuations are computed in the absence of external flow where the equilibrium averages are recovered within the limit $t_{0} \rightarrow-\infty$.

First, the diffusion constant (3.28) of a cluster $\mathscr{N}_{k}$ with $N_{k}$ monomers is computed. It can be expressed in terms of the matrix of second moments of the displacements (4.27) 
according to

$$
\begin{aligned}
D\left(\mathscr{N}_{k}\right): & =\lim _{t \rightarrow \infty} \lim _{t_{0} \rightarrow-\infty} \frac{1}{6 t} \overline{\left[\boldsymbol{R}_{\mathrm{CM}}\left(t \mid \mathscr{N}_{k}\right)-\boldsymbol{R}_{\mathrm{CM}}\left(0 \mid \mathscr{N}_{k}\right)\right]^{2}} \\
& =\lim _{t \rightarrow \infty} \frac{1}{2 t} \frac{1}{N_{k}^{2}} \sum_{i, j \in \mathscr{N}_{k}} D_{i, j}\left(t \mid \mathscr{N}_{k}\right) \\
& =\lim _{t \rightarrow \infty} \frac{1}{2 t} \frac{1}{N_{k}} \operatorname{Tr}\left(E_{0}\left(\mathscr{N}_{k}\right) D\left(t \mid \mathscr{N}_{k}\right)\right) .
\end{aligned}
$$

The benefit of the apparently complicated representation of the diffusion constant in terms of the projector $E_{0}$ will turn out below. The long time asymptotics of the positive matrix $D\left(t \mid \mathscr{N}_{k}\right)$ is dominated by a linear term in $t$ encoding the motion of the center of mass. It is computed in App. C.1 and given by

$$
D\left(t \mid \mathscr{N}_{k}\right) \stackrel{t \rightarrow \infty}{\sim} \frac{2 k_{\mathrm{B}} T}{\zeta} \frac{E_{0}\left(\mathscr{N}_{k}\right) t}{\operatorname{Tr}\left(H^{-1} E_{0}\left(\mathscr{N}_{k}\right)\right)}
$$

Inserting (4.45) into (4.44) and using the projector property $\operatorname{Tr}\left(E_{0}\left(\mathscr{N}_{k}\right) E_{0}\left(\mathscr{N}_{k}\right)\right)=1$ one obtains the diffusion constant

$$
D\left(\mathscr{N}_{k}\right)=\frac{k_{\mathrm{B}} T}{\zeta N_{k}} \frac{1}{\operatorname{Tr}\left(H^{-1} E_{0}\left(\mathscr{N}_{k}\right)\right)}=\frac{k_{\mathrm{B}} T}{\zeta}\left(\sum_{i, j \in \mathscr{N}_{k}}\left[H^{-1}\right]_{i, j}\right)^{-1}
$$

The expression (4.46) for the diffusion constant has been derived in [Oet87b] for the diffusion of linear polymer chains.

Another diffusion constant has been introduced originally by Kirkwood [KiRi48] (cf. [DoEd88, BiCu87]),

$$
D^{(\mathrm{K})}\left(\mathscr{N}_{k}\right):=\frac{k_{\mathrm{B}} T}{\zeta N_{k}^{2}} \sum_{i, j \in \mathscr{N}_{k}} H_{i, j}=\frac{k_{\mathrm{B}} T}{\zeta N_{k}} \operatorname{Tr}\left(E_{0}\left(\mathscr{N}_{k}\right) H\right)
$$

In fact, this expression provides an upper bound to the exact expression (4.46) which can be shown from the representations of the diffusion constants in terms of the projector $E_{0}$ by applying the Jensen-Peierls inequality to (4.46). The simplest version of the JensenPeierls inequality for matrices is given App. F. It implies

$$
\operatorname{Tr}\left(E_{0}\left(\mathscr{N}_{k}\right) H\right) \geq\left[\operatorname{Tr}\left(E_{0}\left(\mathscr{N}_{k}\right) H^{-1}\right)\right]^{-1},
$$

and hence

$$
D^{(\mathrm{K})}\left(\mathscr{N}_{k}\right) \geq D\left(\mathscr{N}_{k}\right)
$$




\subsubsection{Incoherent scattering function}

The incoherent scattering function (3.27) is the characteristic function of the stochastic process $\left(\boldsymbol{R}_{i}(t)-\boldsymbol{R}_{i}(0)\right)$ which is a Gaussian process with zero mean (cf. Eq. (4.26)) and covariance given by (4.27) in the limit $t_{0} \rightarrow \infty$. Thus, the characteristic function reads

$$
S(\boldsymbol{q}, t \mid \mathscr{G})=\frac{1}{N} \sum_{i=1}^{N} \mathrm{e}^{-\frac{q^{2}}{2} D_{i, i}(t \mid \mathscr{G})},
$$

where $q=|\boldsymbol{q}|$ denotest the magnitude of the scattering vector. The expression (4.50) can be written as a sum over contributions from individual clusters

$$
S(\boldsymbol{q}, t \mid \mathscr{G})=\sum_{k=1}^{K} \frac{N_{k}}{N} S\left(\boldsymbol{q}, t \mid \mathscr{N}_{k}\right)
$$

where the cluster contribution is then given by

$$
S\left(\boldsymbol{q}, t \mid \mathscr{N}_{k}\right):=\frac{1}{N_{k}} \sum_{i \in \mathscr{N}_{k}} \mathrm{e}^{-\frac{q^{2}}{2} D_{i, i}\left(t \mid \mathscr{N}_{k}\right)} .
$$

For long times $t \rightarrow \infty$ the scattering function is expected to be dominated by the linear growth of $D_{i, i}\left(t \mid \mathscr{N}_{k}\right)$ with time. More precisely this has to be formulated as an upper bound. By inspection of the long time behavior of $D_{i, i}\left(t \mid \mathscr{N}_{k}\right)$ from App. C. 1 one has

$$
D_{i, i}\left(t \mid \mathscr{N}_{k}\right) \geq \frac{2 k_{\mathrm{B}} T}{\zeta} \frac{\left[E_{0}\left(\mathscr{N}_{k}\right)\right]_{i, i} t}{\operatorname{Tr}\left(H^{-1} E_{0}\left(\mathscr{N}_{k}\right)\right)}=2 D\left(\mathscr{N}_{k}\right) t
$$

for all nodes $i \in \mathscr{N}_{k}$ since the matrix elements $\left[E_{0}\right]_{i, i}=1 / N_{k}$ are equal for all $i \in \mathscr{N}_{k}$. This gives rise to the upper bound

$$
S(\boldsymbol{q}, t \mid \mathscr{G}) \leq \sum_{k=1}^{K} \frac{N_{k}}{N} \mathrm{e}^{-D\left(\mathscr{N}_{k}\right) q^{2} t}
$$

to the incoherent scattering function (4.50). From a physical point of view this is reasonable since it predicts the long time decay of the scattering function to be dominated by the diffusion constants of the clusters.

\subsubsection{Dynamic nonlinear susceptibility}

The nonlinear dynamic susceptibility (3.34) is written as

$$
\chi(\boldsymbol{q}, t \mid \mathscr{G}):=\lim _{t_{0} \rightarrow-\infty} \frac{1}{N} \sum_{i, j=1}^{N} \overline{\mathrm{e}^{\mathrm{i} \boldsymbol{q} \cdot\left[\boldsymbol{R}_{i}(t)-\boldsymbol{R}_{j}(t)\right]} \mathrm{e}^{-\mathrm{i} \boldsymbol{q} \cdot\left[\boldsymbol{R}_{i}(0)-\boldsymbol{R}_{j}(0)\right]}}
$$


and thus identified with the characteristic function of the Gaussian process $\left(\boldsymbol{R}_{i}(t)-\right.$ $\left.\boldsymbol{R}_{i}(0)\right)-\left(\boldsymbol{R}_{j}(t)-\boldsymbol{R}_{j}(0)\right)$ which is the difference of the displacements of monomers $i$ and $j$. In the limit $t_{0} \rightarrow-\infty$ this process is characterized by a zero mean according to (4.26) and a second moment, which can be expressed in terms of the second moments of the displacements (4.27) via

$$
\begin{aligned}
& \overline{\left[\left(\boldsymbol{R}_{i}(t)-\boldsymbol{R}_{i}(0)\right)-\left(\boldsymbol{R}_{j}(t)-\boldsymbol{R}_{j}(0)\right)\right]\left[\left(\boldsymbol{R}_{i}(t)-\boldsymbol{R}_{i}(0)\right)-\left(\boldsymbol{R}_{j}(t)-\boldsymbol{R}_{j}(0)\right)\right]^{\dagger}} \\
& \stackrel{t_{0} \rightarrow-\infty}{=}\left[D_{i, i}(t \mid \mathscr{G})+D_{j, j}(t \mid \mathscr{G})-2 D_{i, j}(t \mid \mathscr{G})\right] \mathbf{1} .
\end{aligned}
$$

Accordingly, the characteristic function (4.55) is given by

$$
\chi(\boldsymbol{q}, t \mid \mathscr{G}):=\frac{1}{N} \sum_{i, j=1}^{N} \mathrm{e}^{-\frac{q^{2}}{2}\left[D_{i, i}(t \mid \mathscr{G})+D_{j, j}(t \mid \mathscr{G})-2 D_{i, j}(t \mid \mathscr{G})\right] .}
$$

The long time asymptotics of the nonlinear susceptibility is of special interest. In the limit $t \rightarrow \infty$ the expression (4.57) can be simplified to an expression of independent contributions of individual clusters. The exponent in (4.57) can be written as a scalar product $\left(\psi^{(i, j)}, D(t \mid \mathscr{G}) \psi^{(i, j)}\right)$ with the vector $\psi_{m}^{(i, j)} \in \mathbb{R}^{\mathbb{N}}$ defined by $\psi_{m}^{(i, j)}=\delta_{i, m}-\delta_{j, m}$ $m=1,2, \ldots N$. For long times $D(t \mid \mathscr{G})$ is dominated by $E_{0}(\mathscr{G}) t / \operatorname{Tr}\left(H^{-1} E_{0}(\mathscr{G})\right)$. If $i$ and $j$ do $n o t$ belong to the same cluster one has $\left(\psi^{(i, j)}, E_{0}(\mathscr{G}) \psi^{(i, j)}\right)>0$ and for $t \rightarrow \infty$ these contributions are suppressed exponentially. If instead $i$ and $j$ do belong to the same cluster one has $\left(\psi^{(i, j)}, E_{0} \psi^{(i, j)}\right)=0$. Accordingly, the sum over all pairs $i, j$ in (4.57) reduces to a sum over all $i, j$ in the same cluster at the cost of a lower bound

$$
\chi(\boldsymbol{q}, t \mid \mathscr{G}) \geq \sum_{k=1}^{K} \frac{N_{k}^{2}}{N} \frac{1}{N_{k}^{2}} \sum_{i, j \in \mathscr{N}_{k}} \mathrm{e}^{-\frac{q^{2}}{2}\left[D_{i, i}\left(t \mid \mathscr{N}_{k}\right)+D_{j, j}\left(t \mid \mathscr{N}_{k}\right)-2 D_{i, j}\left(t \mid \mathscr{H}_{k}\right)\right]} .
$$

This bound can be written as a sum over contributions from individual clusters according to

$$
\chi(\boldsymbol{q}, t \mid \mathscr{G}) \geq \sum_{k=1}^{K} \frac{N_{k}^{2}}{N} \chi\left(\boldsymbol{q}, t \mid \mathscr{N}_{k}\right),
$$

with cluster contributions

$$
\chi\left(\boldsymbol{q}, t \mid \mathscr{N}_{k}\right)=\frac{1}{N_{k}^{2}} \sum_{i, j \in \mathscr{N}_{k}} \mathrm{e}^{-\frac{q^{2}}{2}\left[D_{i, i}\left(t \mid \mathscr{N}_{k}\right)+D_{j, j}\left(t \mid \mathscr{N}_{k}\right)-2 D_{i, j}\left(t \mid \mathscr{N}_{k}\right)\right] .} .
$$




\section{Chapter 5}

\section{Methods}

The following chapter provides methods which shall be employed to compute the critical behavior of dynamical observables within the Rouse and the Zimm model. The dynamics and thus the relaxation times of the system are determined by the eigenvalues of the matrix $\Gamma$ in the Rouse model or by the eigenvalues of the matrix $\widetilde{\Gamma}=H^{\frac{1}{2}} \Gamma H^{\frac{1}{2}}$ in the Zimm model. Hence, computing disorder averages of dynamical observables amounts to the task of computing disorder averaged properties of the eigenvalues of the matrices within the respective ensembles of crosslinks. The methods which will be employed for that purpose differ depending on the properties of the respective matrix. For the Rouse model, the rather discernable structure of the matrix $\Gamma$ facilitates the application of analytical methods. In the Zimm model one is forced to employ numerical methods instead which is due to the more complicated structure of the matrix $\widetilde{\Gamma}$.

However, both matrices share the common property of being block-diagonal which reflects the fact the individual clusters do not interact. This fact considerably simplifies the computation of disorder averages irrespective of the special structure of the matrices and is thus applicable for both models. This cluster decomposition of disorder averages is demonstrated in the following section. The subsequent three sections address properties of the connectivity matrix $\Gamma$ and thus mainly refer to the Rouse model. The last section provides numerical procedures for the matrix $\widetilde{\Gamma}$ in the Zimm model.

\subsection{Disorder averages}

As just mentioned, within the Rouse and the Zimm model all observables such as the viscosity (4.37), the first normal stress coefficient (4.41), the intermediate scattering function (4.51) and the order parameter susceptibility (4.59) share the common property of being decomposable into contributions from individual clusters. The disorder average over different realizations of the crosslink configuration $\mathscr{G}$ of such an observable $A(\mathscr{G})$ will be computed by the following procedure. First, the clusters are sorted according to their size and the average contribution $\langle A\rangle_{n}$ of clusters of a given size $n$ is computed. The occurrence of clusters of a given size $n$ is determined by the cluster size distribution $\tau_{n}$. Thus, 
the overall average $A(\mathscr{G})$ is obtained by weighting the partial averages $\langle A\rangle_{n}$ with the cluster size distribution. This procedure is carried out in the following section and it will give the result

$$
A=\sum_{n=1}^{\infty} n \tau_{n}\langle A\rangle_{n}
$$

for the disorder averaged observable $A$.

\subsubsection{Cluster decomposition of observables}

More precisely, all observables $A(\mathscr{G})$ of interest can be written in the special form

$$
A(\mathscr{G}):=\frac{1}{N} \operatorname{Tr} g(\widetilde{\Gamma}(\mathscr{G}))
$$

where $g$ is a function on the reals. Due to the block diagonal structure of $\widetilde{\Gamma}$ one may decompose $A(\mathscr{G})$ into contributions from different clusters according to

$$
A(\mathscr{G})=\sum_{k=1}^{K} \frac{N_{k}}{N} A\left(\mathscr{N}_{k}\right) .
$$

Here, the contribution of the cluster $\mathscr{N}_{k}$ to $A$ is defined by

$$
A\left(\mathscr{N}_{k}\right):=\frac{1}{N_{k}} \operatorname{Tr} g\left(\widetilde{\Gamma}\left(\mathscr{N}_{k}\right)\right)
$$

In order to compute the disorder average $A:=\langle A(\cdot)\rangle$ as defined in (2.36) over all crosslink realizations $\mathscr{G}$ in the macroscopic limit $M \rightarrow \infty, N \rightarrow \infty$ with fixed crosslink concentration $c:=M / N$ it is useful to introduce partial averages

$$
\langle A\rangle_{n}:=\frac{1}{\tau_{n}}\left\langle\frac{1}{N} \sum_{k=1}^{K} \delta_{N_{k}, n} A\left(\mathscr{N}_{k}\right)\right\rangle
$$

of $A\left(\mathscr{N}_{k}\right)$ over all clusters with $n$ sites. The normalization

$$
\tau_{n}:=\left\langle\frac{1}{N} \sum_{k=1}^{K} \delta_{N_{k}, n}\right\rangle
$$

represents the average number of clusters with $n$ sites per monomer and is nothing but the cluster size distribution. By multiplying Eq. (5.5) with $n^{j} \tau_{n}$ and summing over $n$, one gets the useful identity

$$
\sum_{n=1}^{\infty} n^{j} \tau_{n}\langle A\rangle_{n}=\left\langle\sum_{k=1}^{K} \frac{N_{k}^{j}}{N} A\left(\mathscr{N}_{k}\right)\right\rangle
$$


which is valid in the absence of an infinite cluster. The LHS of Eq. (5.7) describes how the average is computed in terms of the cluster size distribution $\tau_{n}$ and the average over all clusters of size $n$, provided the observable is given by the cluster-decomposed form on the RHS. By setting $j=1$ and comparing with (5.3) the expression

$$
A:=\langle A(\cdot)\rangle=\sum_{n=1}^{\infty} n \tau_{n}\langle A\rangle_{n}
$$

is derived for the disorder average of an observable $A(\mathscr{G})$ with special form $(5.2)$. Equation (5.7) is nothing but the weight average of $A$.

\subsubsection{Critical behavior of observables}

The critical behavior of an observable is determined by the dependence of the disorder average (5.8) on the crosslink concentration $c$, where both $\tau_{n}$ and $\langle A\rangle_{n}$ depend on $c$.

The dependence of $\tau_{n}$ on $c$ is given by the scaling form (2.37). Turning to $\langle A\rangle_{n}$, the dependence on $c$ is more subtle. A percolation cluster of size $n$ can be realized with a different number of bonds. In simple terms, for small $c$ the system comprises only few bonds and the clusters have a more tenuous structure than for larger $c$. This is reflected by the discrimination of lattice animals and percolation clusters (see. Ch. 2.3 [StAh95]). Lattice animals refer to clusters on the percolation lattice for $c \downarrow 0$ whereas percolation cluster refer to clusters at the critical point $c=c_{\text {crit }}$. Lattice animals are thus more tenuous objects than percolation clusters and have a smaller fractal dimension. In principle, the partial average $\langle A\rangle_{n}$ should reflect this crossover from lattice animals to percolation clusters when the critical point is approached. In contrast, for Erdôs-Rényi random graphs the situation is rather simple. The system comprises only trees. Thus, the size $n$ of a cluster fixes the number of vertices and edges, since a tree of size $n$ contains $n-1$ edges. As a conclusion the partial average is independent of $c$ and given by an average over all labelled trees $\mathscr{T}_{n}$ of size $n$

$$
\langle A\rangle_{n}=\frac{1}{n^{n-2}} \sum_{\mathscr{T}_{n}} A\left(\mathscr{T}_{n}\right) .
$$

The normalization $n^{n-2}$ is the number of labelled trees of size $n$. This result stems from the 19th century [Cay89].

However, if one is solely interested in the critical behavior of the series (5.8) the precise dependence of the partial average $\langle A\rangle_{n}$ on the crosslink concentration $c$ is not relevant as long as $\langle A\rangle_{n}$ is regular for $\varepsilon=\left|c-c_{\text {crit }}\right| / c_{\text {crit }} \downarrow 0$ and governed by a power law

$$
A_{n}:=\left.\langle A\rangle_{n}\right|_{\varepsilon=0} \sim n^{b}
$$

at the critical point. This is a reasonable assumption since the self similar system at criticality lacks any characteristic scale. Thus, for $\varepsilon \downarrow 0$ the sum in (5.7) becomes

$$
\sum_{n=1}^{\infty} n^{j-\tau+b} f\left(n \varepsilon^{1 / \sigma}\right),
$$


where the scaling form of the cluster-size distribution has been inserted. As shown in App. E.1 or by virtue of Ch. 2.5 in [StAh95] the critical behavior of (5.11) is given by the scaling form

$$
\sum_{n=1}^{\infty} n^{j} \tau_{n} n^{b} \sim \varepsilon^{-(j+1-\tau+b) / \sigma},
$$

provided, that there is a divergence for $\varepsilon \downarrow 0$, i.e. $j+1-\tau+b>0$. For $j+1-\tau+b<0$ the sum converges to a finite value for $\varepsilon \downarrow 0$ and (5.11) exhibits no divergence at the critical point.

The most important case of the general formula (5.11) is indeed $j=1$, that is the critical behavior of the disorder average (5.8)

$$
A=\sum_{n=1}^{\infty} n \tau_{n} n^{b} \sim \varepsilon^{-(2-\tau+b) / \sigma}
$$

In summary, the computation of the disorder average (5.8) of an observable $A$ reduces to the task of computing the scaling of its partial average (5.10) over clusters of size $n$ at the critical point.

\subsection{Random resistor networks}

In this section a very useful, exact mapping of the viscosity of a Rouse cluster onto a resistor network is derived. This mapping relies on a relation between the Moore-Penrose inverse of the connectivity matrix of the cluster and the resistances in a corresponding resistor network. This mapping yields a simple relation for the viscosity in terms of topological characteristics of the cluster which can be simply read off the molecular graph.

\subsubsection{From Rouse dynamics to random resistor networks}

The dependence of static and dynamic properties of macromolecules on the molecular structure or topology was originally considered in polymer chemistry on a phenomenological level by means of so-called topological indices. Probably the most famous topological index is the Wiener index which was introduced by Wiener in [Wie47]. He recognized that the dependence of boiling points, molar volumes and other properties of alkanes is correlated with the species' molecular topology and well described by the Wiener index. The Wiener index is defined by the sum over all shortest path distances $d_{i, j}$ between all vertices in the molecular graph. For a simple example, see Fig. 5.1. Shortest path distances are called chemical distances and given simply by the number of bonds along the shortest path between $i$ and $j$ along the backbone of the molecular graph.

The Wiener index can be employed to compute static and dynamical properties of treelike macromolecules in the Rouse model. It is a well know fact of graph theory [Mer89] that the sum over the inverse eigenvalues of the Rouse matrix, or equivalently, the trace 


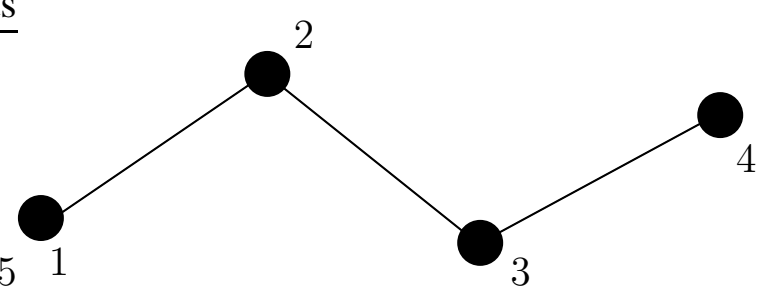

Figure 5.1: Simple example for the Wiener index. For the linear polymer $\mathscr{P}_{4}$ with 4 monomers the Wiener index is $W\left(\mathscr{P}_{4}\right)=d_{1,2}+d_{1,3}+d_{1,4}+d_{2,3}+d_{2,4}+d_{3,4}=10$. For $\mathscr{P}_{n}$ one has $W\left(\mathscr{P}_{n}\right)=n\left(n^{2}-1\right) / 6$.

over the Moore-Penrose inverse of the graph Laplacian, is related exactly to the Wiener index according to

$$
\operatorname{Tr}\left(\frac{\mathbb{1}-E_{0}\left(\mathscr{T}_{n}\right)}{\Gamma\left(\mathscr{T}_{n}\right)}\right)=\frac{1}{2 n} \sum_{i, j \in \mathscr{T}_{n}} d_{i, j}=\frac{W\left(\mathscr{T}_{n}\right)}{n}
$$

The radius of gyration and the viscosity in the Rouse model are both proportional to the sum over inverse eigenvalues. For tree like clusters these quantities can be computed from 5.14 by simply counting chemical distances in the graph [Nit94].

The formulation of the Rouse model in terms of graph theoretical terms has not gained wide acceptance in polymer physics. Only few attempts have been made to carry the formalism of graph theory into the Rouse model, e.g. [Eic72, For76, Gut78] by identifying the connectivity matrix $\Gamma$, originally termed Rouse matrix in polymer physics, with the Laplacian matrix of a graph. By means of graph theory some results for phantom polymers are more easily computed. For instance, the computation of the radius of gyration for general tree like phantom polymers which constitutes the well known Kramer's theorem (see. Ch. 2.4.3 in [RuCo03] is at heart nothing but a particular method of calculating the Wiener index for general tree-like molecules [Mer89].

For molecular topologies which contain loops equation (5.14) is no longer valid if the distances $d_{i, j}$ are regarded as chemical, i.e. shortest-path distances. The generalization of equation (5.14) for arbitrary topology is accomplished by the notion of "resistancedistance" introduced in [KIRa93]. Therefore, a single, connected spring cluster is mapped onto a resistor network by identifying a crosslink between two neighboring monomers with a unit resistor of magnitude $R_{0}$. (see Fig. 5.2) Then, a voltage $U$ is applied to the nodes $i$ and $j$ such that a unit current $I$ is measured. For each vertex $k$ in the graph Kirchhoff's current law $\sum_{l \sim k} I_{l, k}=I\left(\delta_{i, k}-\delta_{j, k}\right)$ must be valid. It states that the sum over the currents $I_{l, k}$ into node $k$ coming from adjacent nodes $l \sim k$ must vanish except for the sink and source vertices $i$ and $j$, respectively. The current in the bond from $l$ to $k$ is related to the difference of vertex potentials $U_{k}-U_{l}$ of these two vertices by Ohm's law $I_{l, k}=\left(U_{k}-U_{l}\right) / R_{0}$. By the definition of the connectivity matrix (2.16) Kirchhoff's law can be rewritten as $\sum_{l} \Gamma_{k, l} U_{l}=R_{0} I\left(\delta_{i, k}-\delta_{j, k}\right)$, where now the sum is over all vertices $l$ in the cluster. The solution of this linear equation for the vertex potentials $U_{l}$ is given by 

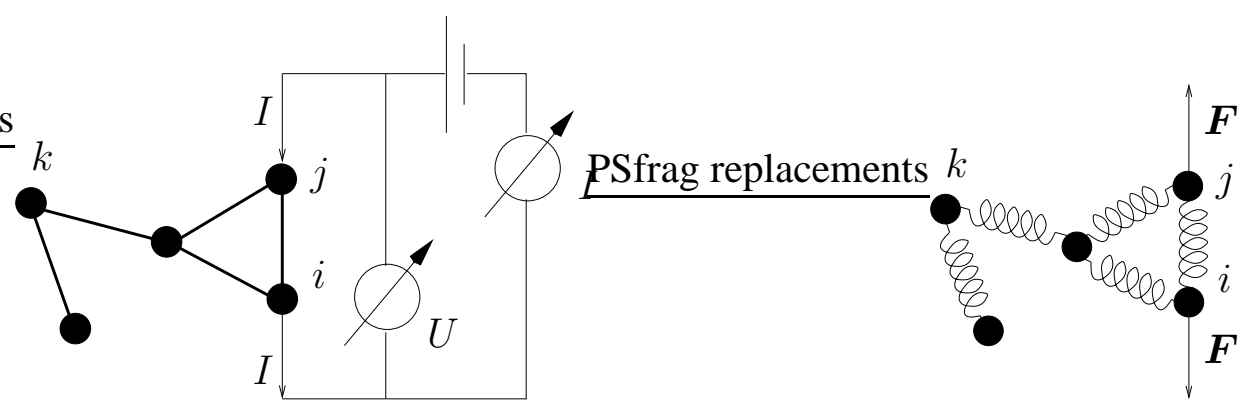

Figure 5.2: Equivalence between the resistor network (left) and a spring network (right). The mechanical analog of Kirchhoff's current law $\sum_{l} \Gamma_{k, l} U_{l}=I R_{0}\left(\delta_{i, k}-\delta_{j, k}\right)$ for vertex $k$ is the balance of forces $3 k_{\mathrm{B}} T / a^{2} \sum_{l} \Gamma_{k, l} \boldsymbol{R}_{l}=\boldsymbol{F}\left(\delta_{i, k}-\delta_{j, k}\right)$ for monomer $k$. Details are explained in the text.

$U_{k}=I R_{0}\left(\left[\left(\mathbb{1}-E_{0}\right) / \Gamma\right]_{k, i}-\left[\left(\mathbb{1}-E_{0}\right) / \Gamma\right]_{k, j}\right)+U_{0}$ with an arbitrary constant $U_{0}$ which stems from the zero eigenvalue of $\Gamma$ and reflects the freedom of choosing the ground of the potential. Hence the potential difference $U=\left(U_{i}-U_{j}\right)$ measured between vertices $i$ and $j$ is given by Ohm's law $U=R_{0} \mathscr{R}_{i, j} I$ with an effective resistance $\mathscr{R}_{i, j}$ which is defined in terms of the matrix elements of the Moore-Penrose inverse

$$
\mathscr{R}_{i, j}=\left[\frac{\mathbb{1}-E_{0}}{\Gamma}\right]_{i, i}+\left[\frac{\mathbb{1}-E_{0}}{\Gamma}\right]_{j, j}-2\left[\frac{\mathbb{1}-E_{0}}{\Gamma}\right]_{i, j} .
$$

Tree cluster contain no loops, and hence no parallel circuits. The resistances on the unique path from vertex $i$ to vertex $j$ are all connected in series and the effective resistance is then simply given by the chemical distance.

In fact, Kirchhoff's law $\sum_{l} \Gamma_{k, l} U_{l}=R_{0} I\left(\delta_{i, k}-\delta_{j, k}\right)$ is the electrical analog of the following mechanical problem. If the spring cluster is subjected to external forces $\boldsymbol{F}$ which act solely on monomers $i$ and $j$ and pull these monomers in opposite directions (see Fig. 5.2), then the equilibrium positions of the monomers $\boldsymbol{R}_{l}$ are determined by the force balance $3 k_{\mathrm{B}} T / a^{2} \sum_{l} \Gamma_{k, l} \boldsymbol{R}_{l}=\boldsymbol{F}\left(\delta_{i, k}-\delta_{j, k}\right)$ of the gradient of the potential energy (2.15) and the external forces. The equation for each component of the vector $\boldsymbol{R}_{l}$ coincides with Kirchhoff's law. Here, the zero eigenvalue of the connectivity matrix allows for an arbitrary, constant vector to be added to the the solution of the force balance equation, which simply reflects the translational invariance. The solution of the mechanical problem $\left(\boldsymbol{R}_{k}-\boldsymbol{R}_{l}\right)=a^{2} /\left(3 k_{\mathrm{B}} T\right) \mathscr{R}_{k, l} \boldsymbol{F}$ is in fact Hooke's law which implies that the inverse effective resistance $\mathscr{R}_{k, l}^{-1}$ is the effective spring constant in the mechanical problem.

For completeness it is remarked that the assumption of identical persistence lengths $a$ and thus identical nearest neighbor resistances $R_{0}$ can be abandoned. If molecules have different, or in general, a distribution of persistence lengths $a_{1}, a_{2} \ldots a_{n}$ Eq. (5.15) remains valid by introducing a weighted Laplacian matrix [KIRa93].

Finally, a useful implication of Eq. (5.15) is given. Since the resistances and the matrix elements of the Moore-Penrose inverse are in a linear one to one correspondence, Eq. 
(5.15) is easily inverted to express the matrix elements in terms of the resistances

$$
\begin{aligned}
{\left[\frac{\mathbb{1}-E_{0}}{\Gamma}\right]_{i, j}=} & -\frac{1}{2} \mathscr{R}_{i, j}+\frac{1}{2 n} \sum_{k=1}^{n} \mathscr{R}_{i, k} \\
& +\frac{1}{2 n} \sum_{k=1}^{n} \mathscr{R}_{k, j}-\frac{1}{2 n^{2}} \sum_{k, l=1}^{n} \mathscr{R}_{k, l}
\end{aligned}
$$

which is valid for an arbitrary connected clusters of size $n$. The proof of equation $(5.16)$ is given in App. B.4.

\subsubsection{How to apply the resistor analogy: Shear viscosity}

The interpretation of $\mathscr{R}_{i, j}$ as a resistance has important consequences for the Rouse model. First, it sheds some light onto the mean squared distance (2.24) between monomers $i$ and $j$ in the same cluster which governs the Gaussian distribution (2.22) of the mutual Euclidean distance: The mean squared distance between nodes $i$ and $j$ in a phantom network is given by its pair resistance in the corresponding resistor network. Accordingly, the radius of gyration $(2.25)$ is simply given by the average resistance between all pairs of vertices in that cluster.

Second, and even more important, the viscosity (4.38) of a cluster in the Rouse model can be related to the resistances. This relation is established by applying the relation (5.15) to the cluster $\mathscr{N}_{k}$ and summing the equation over all $i, j \in \mathscr{N}_{k}$. The matrix elements of the projector $E_{0}\left(\mathscr{N}_{k}\right)$ are all equal to $\left(N_{k}\right)^{-1}$ (see Eq. (2.20) in Sec. 2.3.1). Hence, the sum over all $i, j$ of the last term on the right-hand side equals $-2 N_{k} \operatorname{Tr}\left[E_{0}\left(\mathscr{N}_{k}\right)(\mathbb{1}-\right.$ $\left.\left.E_{0}\left(\mathscr{N}_{k}\right)\right) / \Gamma\left(\mathscr{N}_{k}\right)\right]$ and thus vanishes. This yields

$$
\frac{1}{2 N_{k}} \sum_{i, j \in \mathscr{N}_{k}} \mathscr{R}_{i, j}=\operatorname{Tr}\left(\frac{\mathbb{1}-E_{0}\left(\mathscr{N}_{k}\right)}{\Gamma\left(\mathscr{N}_{k}\right)}\right)
$$

which generalizes the relation (5.14) between the trace of the Moore-Penrose inverse and the Wiener index to arbitrary topologies where the distances $d_{i, j}$ have been replaced by resistances $\mathscr{R}_{i, j}$. The viscosity (4.38) is then directly obtained from (5.17),

$$
\eta\left(\mathscr{N}_{k}\right)=\frac{\zeta a^{2}}{12 N_{k}^{2}} \sum_{i, j \in \mathscr{N}_{k}} \mathscr{R}_{i, j}
$$

It is emphasized that this connection relies on the special form of the viscosity in the Rouse model.

One may test Eq. (5.18) by applying it to a linear polymer with $n$ monomers, i.e. $\mathscr{N}_{k}=$ $\mathscr{P}_{n}$. Then the resistances are connected in series and thus $\eta\left(\mathscr{P}_{n}\right)=\zeta a^{2} / 6 n^{2} W\left(\mathscr{P}_{n}\right)$. As indicated in Fig. 5.1, this gives rise to the well known result $\eta\left(\mathscr{P}_{n}\right)=\zeta a^{2} / 36\left(n^{2}-1\right) / n$ [DoEd88, Ch. 4.5]. 
Finally, the aforementioned connection between the viscosity and the radius of gyration of a cluster in the Rouse model is stated explicitly. By comparing the viscosity (5.18) with the radius of gyration (2.25) the well known result [DoEd88] is found that both quantities are proportional

$$
\eta\left(\mathscr{N}_{k}\right):=\frac{\zeta}{6} R_{\text {gyr }}^{2}\left(\mathscr{N}_{k}\right)
$$

The connection between the radius of gyration and resistances has also been mentioned in [Cat85] without derivation.

The correspondence (5.18) is the fundamental equation for computing the viscosity in the Rouse model, since it allows to relate the critical behavior of the overall viscosity to the critical behavior of the average resistance in a random resistor network. As pointed out in Sec. 5.1 the critical behavior of the viscosity is determined by the scaling with $n$ of the average of the viscosity (4.38) over all clusters of size $n$ at the critical point, viz

$$
\eta_{n}:=\left.\langle\eta(\cdot)\rangle_{n}\right|_{\varepsilon=0} \cdot
$$

Thanks to (5.18) this is nothing but the scaling with $n$ of the average resistance between two vertices in clusters of size $n$ at the critical point.

As pointed out in the beginning of this section, the viscosity is given here as an example how to apply the resistance mapping to Rouse dynamics. It can be applied to other observables as well. Therefore, it is convenient to study the critical scaling of a more general quantity, the $p$-th moment of the pair resistance

$$
\rho_{n}^{(p)}:=\left.\frac{1}{n^{2}} \sum_{i \neq j}^{n}\left\langle\mathscr{R}_{i, j}^{p}\right\rangle_{n}\right|_{\varepsilon=0} .
$$

The viscosity can then be inferred from (5.21) via $\eta_{n}=\left(\zeta a^{2} / 12\right) \rho_{n}^{(1)}$.

For Erdôs-Rényi random graphs the computation of $\rho_{n}^{(p)}$ is a rather simple task. This is due to the fact that trees contain no parallel circuits and resistances reduce to chemical distances such that results on average distances in random trees can be applied [MeMo70]. In contrast, for bond percolation the computation of $\rho_{n}^{(p)}$ is a more complicated issue. However, random resistor networks on percolation clusters have been studied for a long time within mean field approximations [Sti74, Ste77] and renormalization group calculations [HaLu87, HaMe90, StJa99]. The scaling relations which have been obtained for the random resistor networks can be employed to derive the scaling of $\rho_{n}^{(p)}$. This is done in the following section and additionally also other useful quantities from random resistor networks are provided. However, the main result of this rather technical section is the critical scaling of (5.21). The result is given in advance such that the reader is free to go through the details. The scaling of (5.21) for Erdős-Rényi random graphs and bond percolation can be combined in the formula

$$
\rho_{n}^{(p)} \sim n^{p\left(2 / d_{\mathrm{s}}-1\right)},
$$

involving the so-called spectral dimension $d_{\mathrm{s}}$ of critical percolation clusters. The spectral dimension encodes the fractal nature of the fractal without giving reference to its spatial 
configuration and was introduced by Alexander and Orbach [AlOr82] as the fracton dimension. It characterizes the low-energy behavior of the vibrational density of states $N(\gamma)$ of the Laplacian matrix of the critical macroscopic cluster. At the critical point the density of states of the macroscopic cluster is characterized by $N(\gamma) \sim \gamma^{d_{s} / 2-1}$ for small $\gamma \downarrow 0$. This might be regarded as a generalization of the well known behavior of the (phonon) density of states $N(\gamma) \sim \gamma^{d / 2-1}$ of the Laplacian on the regular lattice in spatial dimension $d$. An important point to note about the spectral dimension is the Alexander-Orbach conjecture which states $d_{\mathrm{s}}=4 / 3$ for all spatial dimensions $2 \leq d \leq 6$ [AlOr82]. In fact, the Alexander-Orbach conjecture has not yet been proved wrong but there is a large body of evidence against it (see e.g. [ $\mathrm{HaBe} 02]$ and references therein). Nonetheless, it is a very good approximation for $d=3$ where $d_{\mathrm{s}}=1.33$ is very close but not exactly equal to the mean field value $d_{\mathrm{s}}=4 / 3$. The spectral dimension $d_{\mathrm{s}}$ is the characteristic quantity of a fractal for large chemical distances. It will also emerge in the upcoming sections as a characteristic of random walks on fractals.

\subsubsection{Scaling properties of random resistor networks}

\subsubsection{Erdős-Rényi random graphs}

In Erdôs-Rényi random graphs the computation of resistances is is considerably simpler than for bond percolation. As pointed out in Sec. 5.1 (cf. Eq. (5.9)) the average $\langle\bullet\rangle_{n}$ is over all $n^{n-2}$ equally weighted labelled tree clusters with $n$ monomers. Tree clusters contain no parallel circuits and the resistance between two points is simply given by their mutual chemical distance, that is the number of vertices on the unique path joining $i$ and $j$. The distribution of the chemical distance between two points in a tree chosen randomly from the $n^{n-2}$ labelled trees of size $n$ is known analytically [MeMo70]

$$
p_{\text {dist }}(k):=\left\langle\delta_{\mathscr{R}_{i, j}, k}\right\rangle_{n}=\frac{k+1}{n^{k}} \frac{(n-2) !}{(n-k-1) !}, \quad k=1,2 \ldots n-1 .
$$

Hence, the $p$-th moment (5.21) can be computed in a straightforward manner from the distance distribution (5.23) via

$$
\left\langle\mathscr{R}_{i, j}^{p}\right\rangle_{n}=(n-2) ! \sum_{k=1}^{n-1} \frac{k^{p}(k+1)}{n^{k}(n-k-1) !},
$$

which is independent of $1 \leq i \neq j \leq n$. As shown in App. (E.3), for $n \rightarrow \infty$ the (Riemann-) sum in (5.24) tends to the integral

$$
\begin{aligned}
\left\langle\mathscr{R}_{i, j}^{p}\right\rangle_{n} & \sim n^{p+1} \int_{0}^{1} \mathrm{~d} x x^{p+1} \exp \left[n \int_{0}^{x} \mathrm{~d} y \ln (1-y)\right] \\
& \stackrel{n \rightarrow \infty}{\sim} 2^{p / 2} \Gamma(1+p / 2) n^{p / 2}
\end{aligned}
$$

where in a second step Laplace's method is applied for the asymptotic evaluation of the integral over $x$ for $n \rightarrow \infty$. Euler's gamma function is denoted by $\Gamma$ and $(5.25)$ is valid for $p>-2$. 


\begin{tabular}{l|l|l|} 
& $d=3$ & $d=6$ \\
\hline \hline$\phi$ & 1.12 & 1 \\
\hline$d_{\mathrm{s}}$ & 1.33 & $4 / 3$
\end{tabular}

Table 5.1: Numerical values of the spectral dimension $d_{\mathrm{S}}$ and the resistance crossover exponent $\phi$. The results for $\phi$ and $d_{\mathrm{s}}$ in $d=3$ are taken from [GiLo90] and [HaBe02], respectively.

In summary, for Erdős-Rényi random graphs the $p-t h$ moment (5.21) of the pair resistance is given by

$$
\rho_{n}^{(p)} \stackrel{n \rightarrow \infty}{\sim} 2^{p / 2} \Gamma(1+p / 2) n^{p / 2},
$$

which is valid for all $p>-2$ and includes the prefactor on the LHS of (5.26).

\subsubsection{Bond percolation}

Random resistor networks on percolation clusters have been studied intensively in [Sti74, Ste77, HaLu87, HaMe90, StJa99]. For the purpose of computing $\rho_{n}^{(p)}$ the joint probability $\mu(R, \boldsymbol{x}) \mathrm{d} R$ is considered, that is the probability that two vertices of the random resistor network, whose relative position vector in $\mathbb{R}^{3}$ is $\boldsymbol{x}$, belong to the same cluster and the resistance measured between them lies in the interval from $R$ to $R+\mathrm{d} R$ [HaLu87, StJa99]. It is defined by

$$
\mu(R, \boldsymbol{x})=\left\langle\delta_{\mathscr{N}(i), \mathscr{N}(j)} \delta_{\boldsymbol{x}_{i}-\boldsymbol{x}_{j}, \boldsymbol{x}}, \delta\left(R-\mathscr{R}_{i, j}\right)\right\rangle
$$

where the Kronecker symbol $\delta_{\mathscr{N}(i), \mathscr{N}(j)}$ equals 1 if $i$ and $j$ are in the same cluster and zero otherwise. In the vicinity of the critical point and for large spatial distances $x$ the distribution is given by [HaLu87]

$$
\mu(R, \boldsymbol{x})=\frac{1}{R} x^{-(d-2+\eta)} \mathcal{F}_{\mu}\left(R x^{-\phi / \nu}, x \varepsilon^{\nu}\right)
$$

Here $\mathcal{F}_{\mu}$ is a scaling function and $\nu, \eta$ denote the exponents of lattice bond percolation and $x=|\boldsymbol{x}|$. The equation (5.28) is taken from Eq. (2.39) in [HaLu87] by neglecting corrections to scaling. The exponent $\phi$ is referred to as the crossover resistance exponent of random resistor networks [HaLu87, StJa99] and determines how typical resistances scale with distance, $R \sim|\boldsymbol{x}|^{\phi_{1} / \nu}$. An $\epsilon$-expansion up to second order in $(d-\epsilon), d$ being the spatial dimension, yields [StJa99] the expansion $\phi=1+\epsilon / 42+4 \epsilon^{2} / 3087$. The exponent $\phi$ is related [StAh95] to a more fundamental exponent, the aformentioned spectral dimension, or fracton dimension $d_{\mathrm{s}}$ of percolation clusters (see e.g. Sec. 2.3 in [HaBe02]).

$$
\phi=\frac{1}{\sigma}\left(\frac{2}{d_{s}}-1\right) .
$$

Numerical values of $\phi$ and $d_{\mathrm{s}}$ are given in Tab. 5.1. 
In order to deduce $\rho_{n}^{(p)}$ from the resistance distribution (5.27), the following quantity is considered

$$
\sum_{n=1}^{\infty} n^{2} \tau_{n} \rho_{n}^{(p)}=\left\langle\frac{1}{N} \sum_{k=1}^{K} \sum_{i, j \in \mathscr{N}_{k}} \mathscr{R}_{i, j}^{p}\right\rangle=\left\langle\frac{1}{N} \sum_{i, j=1}^{N} \delta_{\mathscr{N}(i), \mathscr{N}(j)}, \mathscr{R}_{i, j}^{p}\right\rangle
$$

where the first equality is a consequence of (5.7). The last expression in (5.30) can then be simplified by employing translational invariance which amounts to relating it to the resistance distribution $(5.27)$ and determining its critical behavior

$$
\begin{aligned}
\sum_{n=1}^{\infty} n^{2} \tau_{n} \rho_{n}^{(p)} & =\left\langle\sum_{j=1}^{N} \delta_{\mathscr{N}(i), \mathscr{N}(j)} \mathscr{R}_{i, j}^{p}\right\rangle \\
& =\int_{\mathbb{R}^{d}} \mathrm{~d}^{d} x \int_{0}^{\infty} \mathrm{d} R R^{p} \mu(R, \boldsymbol{x}) \\
& \sim \varepsilon^{-(p \phi-(\tau-3)) / \sigma}
\end{aligned}
$$

In fact, (5.31) coincides with Eq. (2.45) in [HaLu87]. The validity of (5.31) requires that there is a divergence for $\varepsilon \downarrow 0$, i.e. that $p \phi-(\tau-3) / \sigma>0$.

It remains to deduce the scaling of $\rho_{n}^{(p)}$ from (5.31). Therefore, a power-law ansatz for the $n$-dependence of $\rho_{n}^{(p)} \sim n^{b_{\rho}(p)}$ is inserted into (5.31). A power law ansatz is a reasonable choice at the critical point in the absence of characteristic scales. By comparing the resulting equation with Eq. (5.12) for $j=2$, one obtains the equality $p \phi-(\tau-3) / \sigma=$ $b_{\rho}(p) / \sigma-(\tau-3) / \sigma$. This yields $b_{\rho}(p)=p \sigma \phi$ or by virtue of the scaling relation (5.29) one has

$$
\rho_{n}^{(p)} \sim n^{b_{\rho}(p)}, \quad \text { with } \quad b_{\rho}(p)=p\left(\left(2 / d_{s}\right)-1\right) .
$$

The prediction (5.32) coincides with Erdős-Rényi random graphs in $d \geq 6$. More precisely, by inserting $d_{\mathrm{s}}=4 / 3$ for $d \geq 6$ the scaling $(5.26)$ is recovered.

Besides the scaling relation (5.32) two other quantities can be inferred from random resistor networks which are useful for computing disorder averages in the Rouse model. As mentioned before, the scaling of the average resistance (5.32) encodes the fractal structure of percolation clusters without referring to the spatial configuration. In other words, it displays properties which should be also encoded in the connectivity matrix $\Gamma$. Therefore, one might discuss the connectivity matrix directly. This was also done in Refs. [HaLu87, StJa99] where the authors discuss the generating function of the distribution of the resolvent of $\Gamma$,

$$
Z(\lambda, \omega):=\left\langle\exp \left\{-\frac{\lambda^{2}}{2}\left(\frac{1}{\Gamma+\mathrm{i} \omega}\right)_{j, j}\right\}\right\rangle .
$$

By means of a renormalization group analysis up to second order in $\varepsilon=6-d$ the validity of the scaling relation

$$
Z(\lambda, \omega) \sim \varepsilon^{\beta} \mathcal{F}_{Z}\left(\varepsilon^{-\phi} \lambda^{2}, \varepsilon^{1 / \sigma} \omega\right)
$$


is shown for $c \uparrow c_{\text {crit. }}$. Moreover, above the percolation transition for $c>c_{\text {crit }}$ the diagonal elements of the Moore-Penrose inverse can be interpreted as the resistance between two infinitely separated points on the macroscopic cluster $\mathscr{N}_{\infty}$ provided $i$ belongs it (see Eq. (2.12) in [StJa99])

$$
R_{\infty}:=2\left[\left(\mathbb{1}-E_{0}\right) / \Gamma\right]_{i, i}, \quad \text { if } i \in \mathscr{N}_{\infty} .
$$

In ([StJa01] $)$ the authors discuss the generating function $Z_{\infty}(\lambda)$ for the resistance between point $i$ and a point at infinity

$$
Z_{\infty}(\lambda):=\left\langle\delta_{\mathscr{N}(i), \mathscr{N}_{\infty}} \exp \left\{-\lambda^{2}\left[\frac{\mathbb{1}-E_{0}}{\Gamma}\right]_{i, i}\right\}\right\rangle .
$$

In the vicinity of the critical point the generating function can be written in the form

$$
Z_{\infty}(\lambda)=S_{\infty} \int_{0}^{\infty} \mathrm{d} x \pi(x) \mathrm{e}^{-\lambda^{2} \varepsilon^{-\phi} / x}
$$

for $c \downarrow c_{\text {crit }}$, where $S_{\infty}$ denotes the gel fraction, i.e. the order parameter of percolation

$$
S_{\infty}=\left\langle\delta_{\mathscr{N}(i), N_{\infty}}\right\rangle= \begin{cases}0 & c<c_{\text {crit }} \\ \varepsilon^{\beta} & c \downarrow c_{\text {crit }}\end{cases}
$$

and $\pi(x)$ is a scaling function. Within a mean field approximation in $d=6$ the Laplace transform $\hat{\pi}(z)=\int_{0}^{\infty} \mathrm{d} x \exp \{-z x\} \pi(x)$ of the scaling function is determined by the nonlinear, second order differential equation

$$
4 z \hat{\pi}^{\prime \prime}(z)=\hat{\pi}(z)(1-\hat{\pi}(z))
$$

with $\hat{\pi}(0)=1$ and $\hat{\pi}(\infty)=0$ (see Eq. (30) in [StJa01]).

\subsection{Random walks on fractals}

A different route to Rouse dynamics of a cluster is accomplished by the equivalence to a particular random walk on the cluster. One might imagine that the propagation of a perturbation applied to one monomer has to diffuse along the backbone of the cluster.

\subsubsection{Connecting Rouse dynamics to a blind ant's random walk}

The connection between Rouse dynamics and random walks is easily motivated formally. The dynamics of a Rouse cluster $\mathscr{N}_{k}$ is quite generally determined by the time evolution matrix $U\left(t \mid \mathscr{N}_{k}\right)=\exp \left\{-3 \Gamma\left(\mathscr{N}_{k}\right) t / t_{\text {mic }}\right\}$, with the microscopic time scale $t_{\text {mic }}$. The fact that the connectivity matrix of a cluster $\mathscr{N}_{k}$ is the discrete Laplacian operator on the corresponding graph amounts to identify the exponential of the connectivity matrix $U\left(t \mid \mathscr{N}_{k}\right)$ 

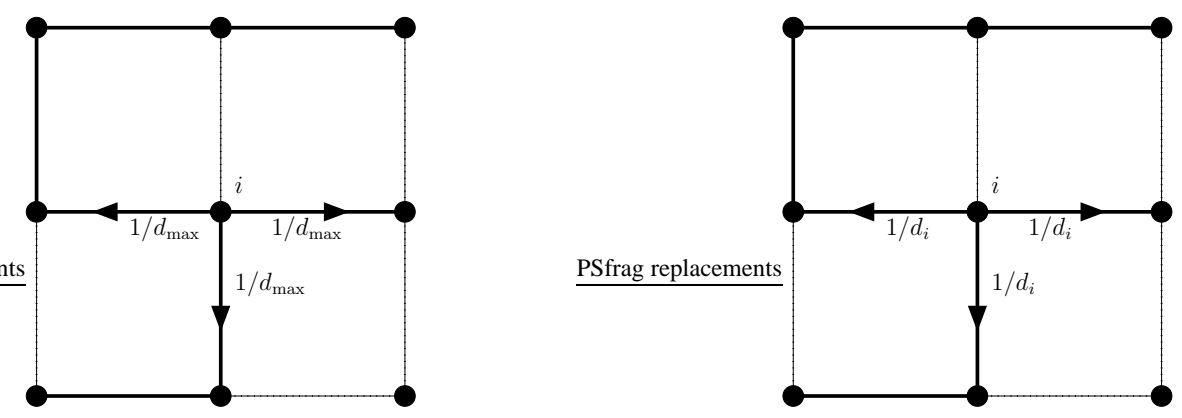

Figure 5.3: Random walk of the blind ant (left) and the myopic ant (right) on a percolation cluster on the square lattice. In this case $d_{i}=3$ and $d_{\max }=4$ (details are given in the text). Thus the blind ant remains at site $i$ with fi nite probability $1 / 4$. This is interpreted as the trial of moving along the nonoccupied bond and the subsequent return after failure.

with the generator of a particular random walk on that cluster. This is the analog to a continuous random walk or diffusion of a particle in $\mathbb{R}^{d}$. If the particle starts at the origin at $t=0$, the transition probability $p_{x}(t)$ satisfies the well known diffusion equation

$$
\partial_{t} p_{\boldsymbol{x}}(t)=\Delta_{\boldsymbol{x}} p_{\boldsymbol{x}}(t)
$$

The formal solution is given by $p_{\boldsymbol{x}}(t)=\exp \left\{t \Delta_{\boldsymbol{x}}\right\} p_{\boldsymbol{x}}(0)$. In this case the Laplace operator $\Delta_{x}$ is said to be the generator of free diffusion.

The connection between Rouse dynamics and random walks on fractals was first used in [Cat84, Cat85] and consequently employed in [Mue03] to derive the scaling behavior of the shear relaxation function $G(t)$ in the Rouse model. This derivation is outlined in the following. A comprehensive review on diffusion in disordered media can be found in [HaBe02].

The diffusion which is generated by $\Gamma$ is referred to as the blind ants' random walk. The term blind ant was introduced by de Gennes [Gen76b] and is distinguished from the so called myopic ant by the choice of one-step probabilities (see below).

Consider a discrete time random walk on an arbitrary connected graph with the restriction that the vertex degree $d_{i}$ of each vertex $i$ must not exceed a maximum value $d_{\max }$. This is true for bond percolation in $d$ dimensions, where the vertex degrees cannot exceed the coordination number $d_{\max }=2 d$ of the lattice $\mathbb{Z}^{d}$. If the ant arrives at site $i$ at time $s$, it moves with equal probability $1 / d_{\max }$ along one of $d_{i}$ adjacent bonds to a neighboring vertex and stays at the same vertex $i$ with probability $1-d_{i} / d_{\max }$. In contrast, the myopic ant moves with equal probability $1 / d_{i}$ to one of the adjacent sites (see Fig. 5.3). By definition of the connectivity matrix $\Gamma$ one has $\Gamma_{i, i}=d_{i}$ and $\Gamma_{i, j}=-1$ if two $i \neq j$ are neighbors and $\Gamma_{i, j}=0$ otherwise. Hence, the blind ant's master equation for the probability $p_{i}(s)$ of visiting vertex $i$ after $s$ time steps is given by

$$
p_{i}(s+1)=\left(1-\Gamma_{i, i} / d_{\max }\right) p_{i}(s)+\sum_{j \neq i}\left(-\Gamma_{i, j} / d_{\max }\right) p_{j}(s)
$$


or equivalently

$$
p_{i}(s+1)-p_{i}(s)=-\frac{1}{d_{\max }} \sum_{j} \Gamma_{i, j} p_{j}(s) .
$$

For $s \gg 1$ the difference on the LHS of (5.42) can be replaced by a derivative. This approximation can be circumvented by defining an appropriate continuous time random walk. The solution of (5.42) with initial condition $p_{i}(0)=\delta_{i, i_{0}}$ is given by $p_{i}(s)=\left[\mathrm{e}^{-s \Gamma / d_{\max }}\right]_{i, i_{0}}$.

The mean return probability for a random walk on critical percolation clusters of size $n$ is then defined by

$$
P_{n}(s):=\left.\left\langle p_{i_{0}}(s)\right\rangle_{n}\right|_{\varepsilon=0}=\left.\left\langle\frac{1}{n} \operatorname{Tr} \mathrm{e}^{-s \Gamma / d_{\max }}\right\rangle_{n}\right|_{\varepsilon=0},
$$

where the independence of the return probability on starting point $i_{0}$ is exploited.

\subsubsection{Scaling properties of the blind ant's random walk}

The scaling of $P_{n}(s)$ for $s \gg 1$ is given by [Mue03]

$$
P_{n}(s) \sim s^{-d_{\mathrm{s}} / 2} \mathcal{F}_{P}\left(s / s_{n}\right)+\frac{1}{n}, \quad \text { with } s_{n} \sim n^{2 / d_{\mathrm{s}}}
$$

and a scaling function $\mathcal{F}_{P}(x)$ which is of order one for $x<1$ and decreases rapidly to zero for $x>1$. The scaling (5.44) can be interpreted as follows. For times $t \lesssim s_{n}$ the ant has not yet recognized the finite nature of the fractal, hence the scaling with time reflects the fractal environment by a power-law. For $t \gtrsim s_{n}$ the ant has roughly discovered the finite fractal completely, and the probability tends to the stationary, uniform distribution $1 / n$. The return probability $P_{\infty}(s)$ on the infinite cluster at the critical point decays according to

$$
P_{\infty}(s) \sim s^{-d_{\mathrm{s}} / 2}
$$

The main result in [Mue03] is to relate the scaling relation (5.43) of the return probability to the scaling of the shear relaxation function (4.36) from clusters of size $n$ at the critical point

$$
G_{n}(t):=\left.\langle G(t \mid \cdot)\rangle_{n}\right|_{\varepsilon=0} .
$$

This is easily done by employing the representation $\left[E_{0}\right]_{i, j}=1 / n$ for $1 \leq i, j \leq n$ of the projector and inserting $d_{\max }=6$ for $d=3$. This yields

$$
G_{n}(t)=k_{\mathrm{B}} T \rho_{\mathrm{m}}\left(P_{n}\left(36 t / t_{\mathrm{mic}}\right)-\frac{1}{n}\right)
$$

Then it is assumed that the overall shear relaxation function $G(t):=\langle G(t \mid \cdot)\rangle$ exhibits a scaling form

$$
G(t) \sim t^{-\Delta} \mathcal{F}_{G}\left(t / t^{*}\right)
$$


in the vicinity of the critical point. The scaling is characterized by an appropriate scaling function $\mathcal{F}_{G}$ with an exponent $\Delta$ and a divergent time scale

$$
t^{*}:=t_{\mathrm{mic}} \varepsilon^{-z} .
$$

By combining the scaling assumption for $G(t)$ with the scaling (5.47) the author in [Mue03] computes the exponent $\Delta$ and the dynamic exponent $z$ in terms of the fundamental exponents $\tau, \sigma, d_{\mathrm{s}}$. They are given by

$$
\Delta=\frac{d_{\mathrm{s}}}{2}(\tau-1), \quad z=\frac{2}{d_{\mathrm{s}} \sigma} .
$$

The deficiency of assuming the scaling form (5.48) instead of deriving it relies on the fact that the scaling form of the average $P_{n}(s)$ and likewise $G_{n}(t)$ is only known precisely at the critical point $\varepsilon=0$, rather than in the vicinity of it.

\subsection{Density of states of the connectivity matrix}

\subsubsection{Connecting Rouse dynamics to the density of states of the graph Laplacian}

The mappings of Rouse clusters onto either resistor networks or random walks on fractals turned out to be useful analogies to characterize certain dynamic quantities in the Rouse model. However, the straightest approach to Rouse dynamics is to compute the density of relaxation times directly. The density of inverse relaxation times, that is the density of eigenvalues of the connectivity matrix is defined by

$$
\begin{aligned}
N(\gamma): & =\left\langle\frac{1}{N} \operatorname{Tr}\left(\left(1-E_{0}(\mathscr{G})\right) \delta(\Gamma(\mathscr{G})-\gamma)\right)\right\rangle \\
& =\frac{1}{\pi} \lim _{\epsilon \downarrow 0} \operatorname{Im}\left\langle\frac{1}{N} \operatorname{Tr} R(-(\gamma+\mathrm{i} \epsilon))\right\rangle .
\end{aligned}
$$

Here $R(\omega):=(\Gamma+\omega \mathbb{1})^{-1}$ is the resolvent of the connectivity matrix and Im denotes the imaginary part of a complex number which stems from the representation of the delta function via the Sokhotsky formula. The shear relaxation function is then simply related to the density of eigenvalues via Laplace transformation

$$
G(t)=k_{\mathrm{B}} T \rho_{\mathrm{m}} \int_{0}^{\infty} \mathrm{d} \gamma N(\gamma) \mathrm{e}^{-\left(6 / t_{\mathrm{mic}}\right) t \gamma} .
$$

Thus, the density of states carries the same information as the shear relaxation function and functional properties of the density of states can be translated into functional properties of the shear relaxation function. 


\subsubsection{Scaling properties of the density of states}

The connectivity matrix is a random matrix, with a distribution of eigenvalues which is not given by Wigner's famous semicircle law [Wig58]. In fact, for Erdős-Rényi random graphs it is possible [BrAs01] to derive a nonlinear integral equation for the density of states (5.51) by means of a replica representation of the resolvent $R(\gamma)$. As a result, the density of states $N(\gamma)$ can be computed exactly for arbitrary $\gamma$ by an iterative procedure. Additionally, for small $\gamma$ the lowest order, inverse moments of the density of states can be computed exactly [BrAs01]

$$
\begin{gathered}
M_{1}:=\int_{0}^{\infty} \mathrm{d} \gamma \frac{N(\gamma)}{\gamma}=\frac{1}{4 c}\left[\ln \left(\frac{1}{1-2 c}\right)-2 c\right] \\
M_{2}:=\int_{0}^{\infty} \mathrm{d} \gamma \frac{N(\gamma)}{\gamma^{2}} \\
=c\left[-\frac{8 c^{3}-6 c^{2}-5 c+1}{30 c(1-2 c)^{3}}-\frac{4 c^{2}-3 c-1}{24 c(1-2 c)^{2}}+\frac{1}{240 c^{2}} \ln (1-2 c)\right] .
\end{gathered}
$$

Even more interesting, the density of eigenvalues exhibits a Lifshits tail, that is a singular fall off for $\gamma \downarrow 0$. Lifshits tails in the density of states in disordered systems can be related to the occurrence of very small, exponentially rare eigenvalues. Bray and Rodgers [BrRo88] were the first to propose that the Lifshits tail can be traced back to the smallest eigenvalues of all clusters of size $n$ which stems from the linear cluster and is of order $1 / n^{2}$. In [BrAs01] it is then proved that the density of states exhibits a Lifshits tail

$$
N(\gamma) \sim \exp \left\{-\left(\gamma^{*} / \gamma\right)^{\alpha_{N}}\right\}, \quad \gamma \downarrow 0, \quad c<c_{\text {crit }},
$$

with an exponent $\alpha_{N}=1 / 2$ and a typical scale $\gamma^{*}$. The Lifshits tail was actually proved for the integrated density of states which should, however, also apply to the density of states itself if algebraic prefactors are neglected. Thus, in [BrAs01] the scaling (5.56) for small $\gamma$ is translated into the leading behavior of the shear relaxation function (5.53) for large $t$

$$
G(t) \sim \exp \left\{-\left(t / t^{*}\right)^{\alpha_{G}}\right\}, \quad t \rightarrow \infty, \quad c<c_{\text {crit }},
$$

with an exponent $\alpha_{G}=1 / 3$ and a time scale $t^{*} \sim 1 / \gamma^{*}$. From the behavior of the lowest order, inverse moments it is suggested that $t^{*} \sim 1 / \gamma^{*} \sim \varepsilon^{-3}$. This claim is in accordance with the scaling (5.49) for mean field values $\sigma=1 / 2$ and $d_{\mathrm{s}}=4 / 3$. Unfortunately, an a posteriori verification of the assumed scaling behavior (5.48) is not possible from the results obtained for the density of states since algebraic prefactors in (5.57) remain undetermined.

For bond percolation the density of states of the connectivity matrix $\Gamma$ on percolation clusters has become a focus of interest in the mathematical literature. In [KiMu04] it is rigorously shown that the integrated density of states of $\Gamma$ exhibits a Lifshits tail with the same exponent $\alpha_{D}=1 / 2$ as in (5.56). However, if the integrated density of states exhibits 
a Lifshits tail so does (5.51) and the scaling form (5.57) for the long time behavior of the shear relaxation function is valid for all spatial dimensions $d$ with the same exponent $\alpha_{G}=1 / 3$. In contrast, the scale $t^{*} \sim 1 / \gamma^{*}$ does depend on the dimensionality $d$ but its $\varepsilon$-dependence cannot be derived within the approach [KiMu04].

All three, apparently different approaches to Rouse dynamics from the previous chapters involve the spectral dimension $d_{\mathrm{s}}$ as the characteristic exponent and therefore it is worthwhile to stress the connections between these approaches. The density of states may be regarded as the fundamental quantity in the Rouse model. The Laplace transform of the density of states of clusters of size $n$ equals the return probability of the blind ant's random walk on clusters of size $n$. It remains to establish a connection to random resistor networks. For the myopic ant it is a well known fact that the effective resistance $\mathscr{R}_{i, j}(5.15)$ is proportional to the commute time between vertices $i$ and $j$ [Tet91]. The commute time is the expected time it takes the myopic ant to start at vertex $i$, visit vertex $j$ and return to $i$. However, the return probability of both ants is characterized by the same exponent $d_{\mathrm{s}}$ for large times [Mar88, HaMe87]. Therefore, the effective resistance $\mathscr{R}_{i, j}$ also determines the commute time for the blind ant's random walk at least for vertices $i, j$ which are a large chemical distance apart. This demonstrates why the scaling of the effective resistances is also characterized by the spectral dimension.

As an aside, the interpretation of the resistance as a commute time allows characterizing recurrence properties of random walks by an illustrative and exact picture [Tet91]: The walk is recurrent if the effective resistance between the starting point and a "point at infinity" (cf. Eq. 5.35) is infinite.

\subsection{Numerical methods}

Within the Zimm model it is not possible to establish a relation between the generalized connectivity matrix $\widetilde{\Gamma}$ and topological properties, which would allow to treat Zimm dynamics analytically. Hence, one is forced to compute the averages (5.10) of observables over clusters of size $n$ and the corresponding scaling exponent $b$ numerically in order to obtain the critical behavior from (5.11).

The numerical computation of partial averages $\langle\bullet\rangle_{n}$ requires three subsequent steps. First, an algorithm is required that generate clusters (graphs) $\mathscr{N}_{k}$ of size $n$ according to their probability of occurrence in their respective ensembles. Second, for each generated cluster $\mathscr{N}_{k}$ the connectivity matrix $\Gamma\left(\mathscr{N}_{k}\right)$ and the mobility matrix $H\left(\mathscr{N}_{k}\right)$ have to be

computed in order to build the generalized connectivity matrix $\widetilde{\Gamma}\left(\mathscr{N}_{k}\right)$. Third, observables are computed from spectral properties of $\widetilde{\Gamma}\left(\mathscr{N}_{k}\right)$ by a numerical diagonalization of $\widetilde{\Gamma}\left(\mathscr{N}_{k}\right)$. These three steps slightly differ, depending on which crosslink ensemble is used. Therefore Erdôs-Rényi random trees and bond percolation are discussed separately. 


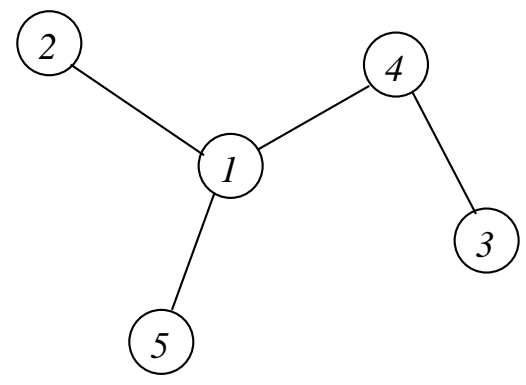

\begin{tabular}{c||c|c|c|c|c||c}
\multicolumn{8}{c}{$S_{\mathrm{P}}=\left\{v_{1}, v_{2}, v_{3}\right\}=\{1,4,1\}$} \\
\multicolumn{1}{l||}{$d_{1}$} & $d_{2}$ & $d_{3}$ & $d_{4}$ & $d_{5}$ & new edge \\
\hline \hline 1 & 3 & 1 & 1 & 2 & 1 & $(2,1)$ \\
\hline 2 & 2 & 0 & 1 & 2 & 1 & $(3,4)$ \\
\hline 3 & 2 & 0 & 0 & 1 & 1 & $(4,1)$ \\
\hline & 1 & 0 & 0 & 0 & 1 & $(1,5)$ \\
\hline
\end{tabular}

Figure 5.4: Example for the Prüfer algorithm for a given Prüfer sequence $S_{\mathrm{P}}$ and tree size $n=5$. The table shows the values of the degree sequence $d_{1}, d_{2} \ldots d_{5}$ at the beginning of the $l$ th main loop and the corresponding new edge which is created at the end of the $l$ th loop.

\subsubsection{Erdős-Rényi random graphs}

As already mentioned in Sec. 5.1.2, for the Erdős-Rényi random graphs the average $\langle\bullet\rangle_{n}$ is an average over all $n^{n-2}$ equally weighted, labelled trees of size $n$. Labelled trees of a given size can easily generated via the Prüfer algorithm [Pru18]. It provides a mapping from a sequence $S_{\mathrm{P}}=\left\{v_{l}\right\}_{l=1}^{n-2}$ of $n-2$ independent, identically distributed random integer variables, each of which is distributed uniformly on the integer set [1..n]. The $v_{l}$ can be regarded as the vertices of the tree. Obviously, there are $n^{n-2}$ possible sequences, which are in a one-to-one correspondence with the set of trees of size $n$ according to the following (Prüfer) algorithm

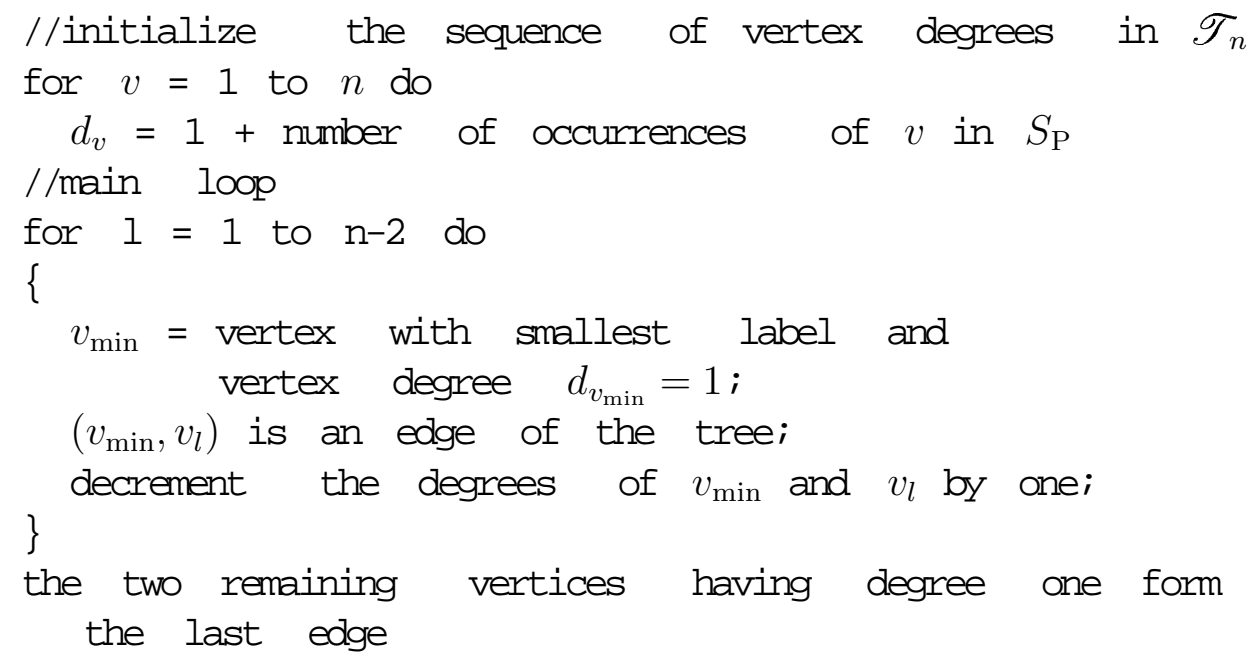

The algorithm is demonstrated for a simple example in Fig. (5.5.1) The overall computer time for the simple implementation described above is $\mathscr{O}\left(n^{2}\right)$. If the vertices are held in a priority queue, the operation of dequeueing the item with highest priority, i.e. the vertex with the smallest label, is of order $\mathscr{O}(\log n)$ [MeNa99, Ch. 5.4] and hence the numerical complexity of the Prüefer algorithm is of order $\mathscr{O}(n \log n)$. 


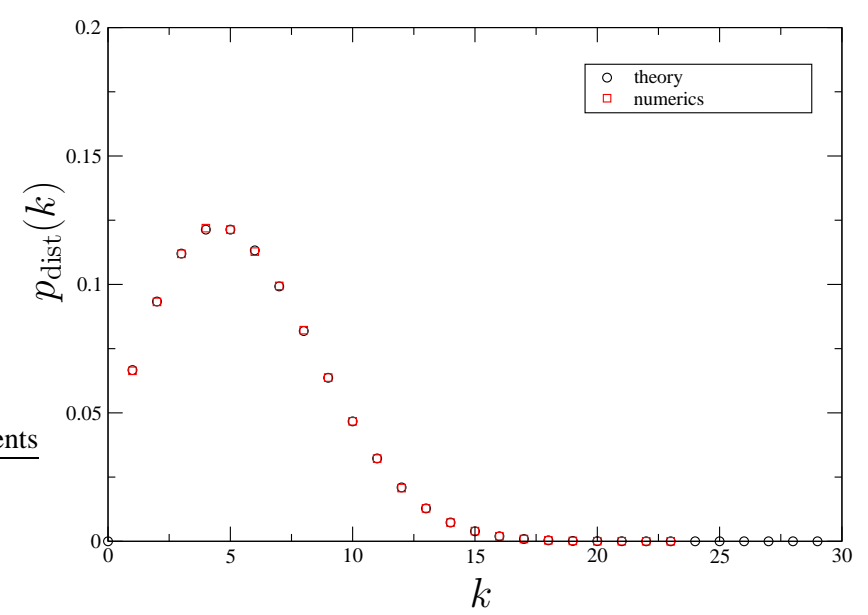

Figure 5.5: Distribution of the resistance (distance) between two arbitrary vertices in a tree of size $n=30$.

The output of the Prüfer algorithm is an adjacency list of $n$ vertices of the tree which is handled with the LEDA-library [MeNa99]. The library supports common data structures associated with graphs. After the generation of a tree $\mathscr{T}_{n}$ the connectivity matrix $\Gamma\left(\mathscr{T}_{n}\right)$ is computed from the adjacency list according to Eq. (2.16).

The computation of the preaveraged mobility matrix (2.29) requires the pair resistances $\mathscr{R}_{i, j}$. As already mentioned the resistance between the vertices $i$ and $j$ in the tree $\mathscr{T}_{n}$ is simply the chemical distance between them, that is the length of the shortest (and unique) path. These distances can be computed with the Dijkstra algorithm which is provided by LEDA and is of complexity $\mathscr{O}\left(n^{2}\right)$. The preaveraged mobility matrix $H\left(\mathscr{T}_{n}\right)$ is then computed with the function $h$ from (2.31), corresponding to the Rotne-Prager tensor. After these steps $H\left(\mathscr{T}_{n}\right)$ and $\Gamma\left(\mathscr{T}_{n}\right)$ are available. The complexity of the generation of these matrices as a function of tree size $n$ is of order $O\left(n^{2}\right)$ due to the computation of the distances.

Tests The numerical computation of resistances can be tested by comparing the numerical distribution of the resistance (distance) $\mathscr{R}_{i, j}$ for an arbitrary pair of vertices $i, j$ with the analytical expression (5.23). In Fig. 5.5 the distribution is computed from $10^{6}$ tree realizations for $n=30$ and compared with the theoretical prediction. Deviations from the theoretical distribution are negligible. This is quite remarkable when the sample size $10^{6}$ is compared with the total number $30^{28} \approx 10^{41}$ of trees of size $n=30$.

\subsubsection{Bond percolation}

For the generation of clusters according to 3-dimensional percolation there are mainly two possibilities. Within the box filling method bonds are thrown in a finite, cubic sublattice of $\mathbb{Z}^{d}$ for different sizes of the cube. The infinite volume properties are then obtained by 
finite size scaling.

However, for an investigation of single cluster properties the Leath method [Lea76] is more advantageous. The Leath algorithm generates single clusters, by starting from an initial seed vertex which may be regarded as an arbitrary point of the hypercubic lattice. All points on the lattice which are adjacent to the seed are marked as "connected" with probability $p$ or marked as "disconnected" with probability $1-p$. This procedure is repeated recursively for all points which already have been marked as connected until all connected points are surrounded by disconnected points.

In order to compute disorder averages, the average $\langle\bullet\rangle_{n}(5.5)$ has to be computed for a sequence $\left\{\mathscr{L}_{i}\right\}_{i=1}^{L}$ of generated Leath clusters. The correspondence between the output of the algorithm and the disorder average $A$ of an observable which can be decomposed into different clusters according to (5.2) is given by

$$
A=\lim _{L \rightarrow \infty} \frac{1}{L} \sum_{l=1}^{L} A\left(\mathscr{L}_{l}\right)
$$

as shown in App. G.

By choosing $A\left(\mathscr{N}_{k}\right):=\delta_{N_{k}, n} / N_{k}$ the cluster size distribution $\tau_{n}$ is obtained which can be seen by virtue of Eqs. (5.7) and (5.6). Accordingly, the cluster size distribution $\tau_{n}$ is obtained from the Leath average (5.58) via

$$
\begin{aligned}
\tau_{n} & =\frac{1}{n} \lim _{L \rightarrow \infty} \frac{1}{L} \sum_{l=1}^{L} \delta_{N\left(\mathscr{L}_{l}\right), n} \\
& =: \frac{1}{n} \tau_{n}^{\text {(Leath) }} .
\end{aligned}
$$

The implication of Eq. (5.59) is obvious: the cluster size distribution $\tau_{n}^{\text {(Leath) }}$ of the Leath algorithm differs by a factor $n$ from the cluster size distribution of the box filling method. Hence, the number of clusters of size $n$ generated by the Leath method is by a factor $n$ larger as for the box filling method. This is particularly useful for studying critical behavior which is dominated by clusters with large $n$.

For a given Leath cluster $\mathscr{L}$ the resistances $\mathscr{R}_{i, j}$ are computed from the Moore-Penrose inverse of the connectivity matrix $\Gamma(\mathscr{L})$ according to Eq. (5.15). The Moore-penrose inverse is computed via the spectral decomposition ( $\overline{\mathrm{B} .7})$, where the eigenvalues and eigenvectors have been obtained with the LAPACK-routine dsyev_ . The smallest numerically computed eigenvalue, which is small but numerically nonzero, is taken to be the null eigenvalue of $\Gamma(\mathscr{L})$. The preaveraged mobility matrix $(2.29)$ is computed with the function $h$ from (2.31), corresponding to the Rotne-Prager tensor. After these steps, the matrices $\Gamma(\mathscr{L})$ and $H(\mathscr{L})$ are available. The numerical complexity is of order $\mathscr{O}\left(n^{3}\right)$ due to the spectral decomposition of the connectivity matrix.

Tests The algorithm is tested by comparing the numerical data with literature results for bond percolation. According to Stauffer [StAh95] the cluster size distribution $\tau_{n}$ obeys the 


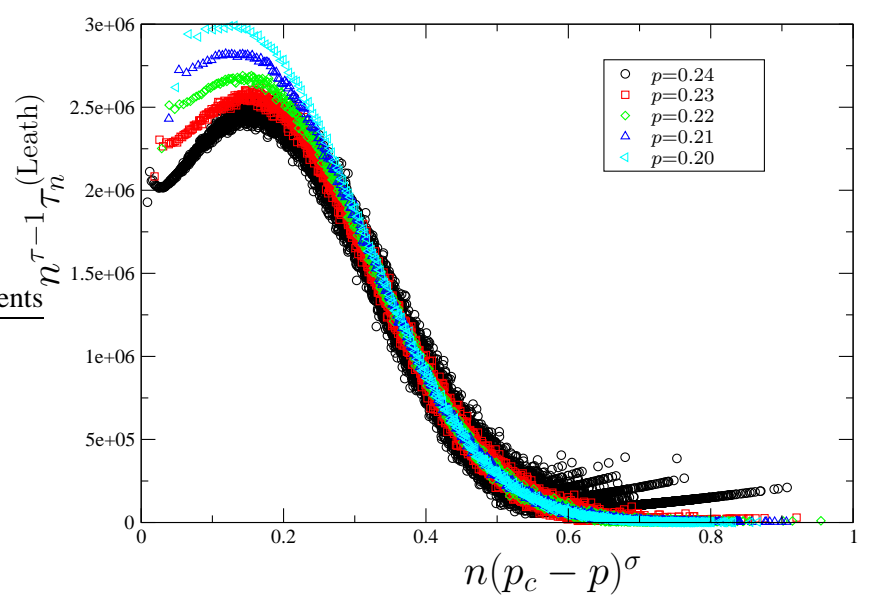

Figure 5.6: Scaling of the cluster size distribution with literature values $\tau=2.18$ and $\sigma=0.45$ taken from [StAh95]

scaling law (1.3) for large $n$ and bond probabilities $p$ close to the percolation probability $c_{\text {crit }}=0.2488$. The scaling assumption predicts a master curve for large $n$ if $n^{\tau} \tau_{n}$ is plotted as a function of the scaling variable $n \varepsilon^{\sigma}$. In Fig. (5.6) $n^{\tau-1} \tau_{n}^{\text {(Leath) }}$ is plotted as a function of $n \varepsilon^{\sigma}$. The values $\sigma=0.45$ and $\tau=2.18$ for the exponents for $3 d$ bond percolation are taken from the literature [StAh95]. For large $n$ the curves for different values of the bond probability $p$ fall fairly well on top of each other. The scattering of the data for $p=0.24$ displays the fact that for large $n$ only very few, i.e. $1,2 \ldots$ clusters per cluster size are generated. The total number $10^{7}$ of generated clusters is still to small to obtain accurate statistics at high $n$ which reflects the power law decay of the cluster size distribution at the critical point.

As a second test the numerical data is compared to exact series expansion results of perimeter polynomials $D_{m}(1-p)$. The perimeter polynomials $D_{m}(1-p)$ are defined by the relation $[\mathrm{SyGa} 81]$

$$
K(p):=\sum_{m=1}^{\infty} D_{m}(1-p) p^{m}
$$

for the mean number $K(p)$ of finite clusters per lattice site at density $p$ where the sum is over all possible numbers of bonds. This implies that $n_{m}(p):=D_{m}(1-p) p^{m}$ is the mean number of clusters per lattice site consisting of $m$ bonds and an arbitrary number of vertices. The presence of $m$ bonds is reflected by the factor $p^{m}$ and the average geometry of the cluster is characterized by the perimeter polynomial $D_{m}(1-p)$. For the Leath algorithm, one has [PiSt81]

$$
n_{m}^{(\text {Leath })}(p):=\frac{1}{d} \frac{m}{p} n_{m}(p)
$$

which allows comparing the numerically obtained value $n_{m}^{\text {(Leath) }}(p)$ with the analytical expression $n_{m}(p)$ for the simple cubic lattice from [SyGa81]. The numerical data is shown 


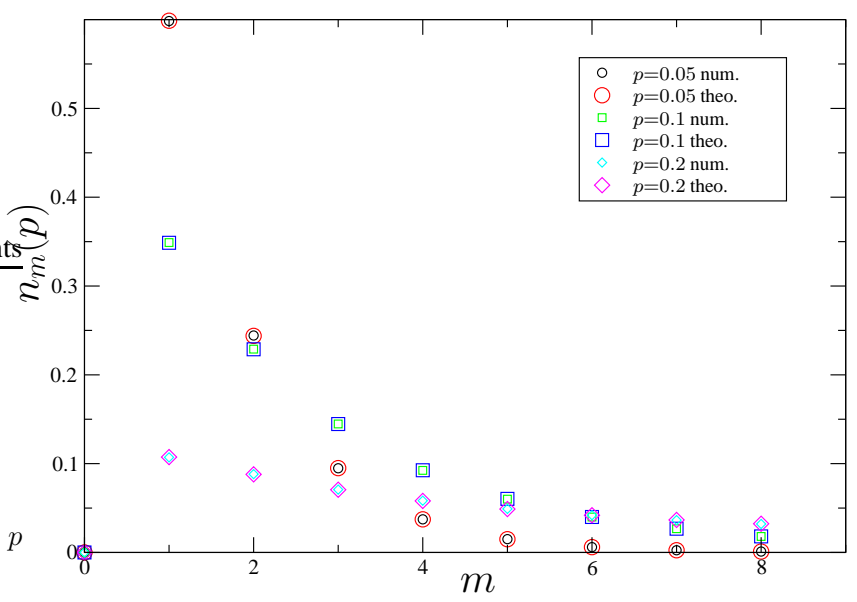

Figure 5.7: Average number $n_{m}(p)$ of clusters with $m$ bonds for different values of $p$. Comparison of the numerical results with those obtained from series expansions for the perimeter polynomials (for details see text).

in shown in Fig. 5.7, it is excellent agreement with the analytical expression for various values of $p$.

In the last test the scaling of a typical, averaged single cluster quantity with cluster size $n$ is computed, namely the scaling of the squared radius of gyration $R_{\mathrm{gyr}, n}^{2}$ of lattice percolation clusters at the critical point.

Since each Leath cluster $\mathscr{L}_{l}$ is generated on the cubic lattice $\mathbb{Z}^{3}$ the radius of gyration is defined by

$$
R_{\text {gyr }}^{2}\left(\mathscr{L}_{l}\right):=\frac{1}{2\left(N\left(\mathscr{L}_{l}\right)\right)^{2}} \sum_{i, j \in \mathscr{L}_{l}}\left(\boldsymbol{x}_{i}-\boldsymbol{x}_{j}\right)^{2}
$$

where $\boldsymbol{x}_{i}-\boldsymbol{x}_{j}$ is the Euclidean distance vector between points $i, j$ on that lattice in units of the nearest neighbor distance. At the critical point $p_{\mathrm{c}} \approx 0.2488$ the radius of gyration is computed as a function of the cluster size $n$. The result is shown in Fig. 5.8. By fitting the data to a power law $R_{\mathrm{gyr}, n}^{2} \sim n^{b_{\mathrm{R}}}$ in the interval $[50,5000]$ the exponent $b_{\mathrm{R}} \approx 0.791$. This is to be compared with Eq. (1.8). The literature value $d_{\mathrm{f}}^{(\mathrm{H})}=2.53$ yields $b_{\mathrm{R}}=2 / d_{\mathrm{f}}^{(\mathrm{H})} \approx$ $2 / 2.53 \approx 0.791$ which is in perfect agreement with the above result. 


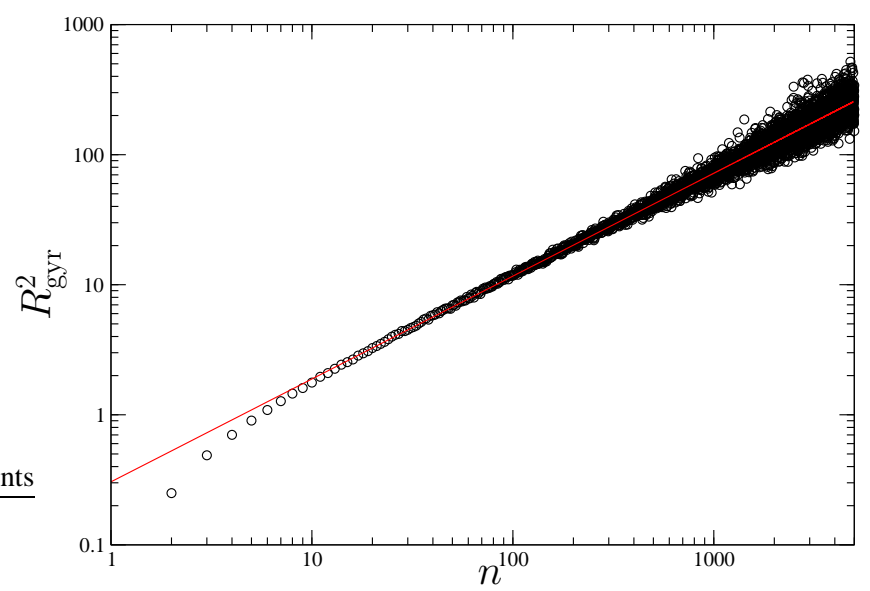

Figure 5.8: Scaling of the squared radius of gyration of percolation clusters at the critical point as a function of size $n$. 


\section{Chapter 6}

\section{Results}

\subsection{Rouse Model}

Observables in the Rouse model are obtained from the formulas derived in Secs. 4.2 and 4.3 by setting $H=\mathbb{1}$.

\subsubsection{Shear viscosity}

Even though briefly explained in section 5.2, here the procedure of obtaining the averaged viscosity starting from the expression (4.36) is demonstrated again since these steps are important and identical for all observables.

The disorder average $\eta=\langle\eta(\cdot)\rangle$ of the shear viscosity $\eta(\mathscr{G})$ from (4.36) is computed by employing Eq. (5.8) which amounts to

$$
\eta=\sum_{i=1}^{\infty} n \tau_{n} \eta_{n}
$$

Thereby the partial average $\langle\eta(\cdot)\rangle_{n}$ in (5.8) has been replaced by the average $\eta_{n}$ (see Eq. (5.20) at the critical point which suffices to compute the critical behavior of $\eta$ as explained in Sec. 5.1.2. The scaling of $\eta_{n}$ is deduced from Eqs. (5.18), (5.20) and (5.21), yielding

$$
\eta_{n}=\frac{\zeta a^{2}}{12} \rho_{n}^{(1)} .
$$

For Erdős-Rényi random graphs an exact expression for the averaged viscosity valid for all $c<c_{\text {crit }}$ was already derived in [BrL099, Loe99]. This was done by inserting the exact expression (5.24) for $\rho_{n}^{(1)}$ into (6.1). The series can be summed up by analytic means and gives

$$
\eta=\frac{\zeta a^{2}}{24 c}\left[\ln \left(\frac{1}{1-2 c}\right)-2 c\right] .
$$

Hence, for Erdős-Rényi random graphs the viscosity diverges logarithmically for $c \uparrow c_{\text {crit }}$, corresponding to a critical exponent $k=0$. Additionally, the result $(6.3)$ can be rederived 
[BrLo01b] by expressing the viscosity $\eta$ in terms of the first, inverse moment $M_{1}$ (5.54) of the density of states (5.51) according to $\eta=\frac{\zeta a^{2}}{6} M_{1}$. Again, one ends up with (6.3).

For bond percolation the averaged contribution (6.2) of the viscosity of clusters of sizes $n$ is determined by the scaling form

$$
\eta_{n}:=\left.\langle\eta(\cdot)\rangle\right|_{\varepsilon=0}=\frac{\zeta a^{2}}{12} \rho_{n}^{(1)} \sim n^{2 / d_{s}-1}
$$

as a consequence of Eqs. (5.18),(5.21) and (5.32). The result (6.4) is an exact expression for the Mark-Houwink relation of percolation clusters in the Rouse model at the critical point. The same scaling relation has been obtained in [Mut85] by generalizing the Rouse equation of motion of a linear chain heuristically to fractal topologies.

The critical behavior of the overall viscosity (6.1) for bond percolation follows from Eqs. (6.4) and (5.13) and is given by

$$
\eta \sim \varepsilon^{-k}, \quad \text { with } \quad k=\left(2 / d_{\mathrm{s}}+1-\tau\right) / \sigma .
$$

It agrees with (6.3) for Erdős-Rényi random graphs if the mean field exponents $(d \geq 6)$ for bond percolation are inserted. The numerical values for $k$ are inferred from Tabs. 1.2, 5.1. This implies $k=0$ in $d=6$ which corresponds to the logarithmic divergence $(6.3)$ and $d=3$ one has $k=0.71$.

The derivation of the result (6.5) relies on the assumption (5.32) that the $p$-th moment follows a power law at the critical point which is reasonable but could not be justified rigorously. However, this assumption can be confirmed a posteriori. Therefore the viscosity $\eta$ is related to the generating function $Z(\lambda, \omega)$ of the resolvent of $\Gamma$ which is defined by Eq. (5.33). Using the fact that the system is translationally invariant the viscosity (4.36) averaged over the disorder is related to $Z(\lambda, \omega)$ via

$$
\begin{aligned}
\eta & =\frac{\zeta a^{2}}{6}\left\langle\left(\frac{1-E_{0}}{\Gamma}\right)_{j, j}\right\rangle \\
& =-\left.\frac{\zeta a^{2}}{3} \lim _{\omega \rightarrow 0} \operatorname{Re} \frac{\partial}{\partial \lambda^{2}}\right|_{\lambda=0} Z(\lambda, \omega) .
\end{aligned}
$$

Here, for the second equality the expansion (B.6) of the resolvent of $\Gamma$ is employed in. From (6.6) the result (6.5) is readily re-derived.

In order to shed some light onto the viscosity scaling (6.4) the relation of the radius of gyration of percolation clusters is re-examined. Since $\eta\left(\mathscr{N}_{k}\right):=(\zeta / 6) R_{\text {gyr }}^{2}\left(\mathscr{N}_{k}\right)$ (cf. (5.19)) the averaged radius of gyration $R_{\text {gyr }, n}^{2}$ of percolation clusters with $n$ monomers follows the same scaling form as the viscosity

$$
R_{\text {gyr }, n}^{2}=\left.\left\langle R_{\text {gyr }}^{2}(\cdot)\right\rangle_{n}\right|_{\varepsilon=0} \sim n^{2 / d_{\mathrm{s}}-1} .
$$

When comparing Eq. (6.7) to the familiar relation $R_{n}^{2} \sim n^{2 / d_{\mathrm{f}}^{(\mathrm{H})}}$ (cf. Eq. 1.8) for the radius of gyration of lattice percolation clusters in terms of the Hausdorff fractal dimension $d_{\mathrm{f}}^{(\mathrm{H})}$ 
it is suggestive to introduce a Gaussian fractal dimension $d_{\mathrm{f}}^{(\mathrm{G})}$ by

$$
\frac{2}{d_{\mathrm{f}}^{(\mathrm{G})}}:=\left(\frac{2}{d_{s}}-1\right)
$$

for Gaussian percolation clusters [Cat85]. By means of $d_{\mathrm{f}}^{(\mathrm{G})}$ the scaling of the radius of gyration (6.7) and the viscosity (6.4) is rewritten as

$$
R_{\mathrm{gyr}, n}^{2} \sim \eta_{n} \sim n^{2 / d_{\mathrm{f}}^{(\mathrm{G})}} .
$$

The Gaussian fractal dimension for randomly branched polymers was first computed by Zimm and Stockmayer [ZiSt49] and is given by $d_{\mathrm{f}}^{(\mathrm{G})}=4$.

Finally, it is mentioned that the critical behavior of the viscosity $(6.5)$ can also be deduced from $\eta=\int_{0}^{\infty} \mathrm{d} t^{\prime} G\left(t^{\prime}\right)$ by employing the scaling of the shear relaxation function $G(t)$ from (5.48) [Mue03]. It yields $k=z(1-\Delta)$ and by virtue of the definitions of the exponents $z, \Delta$ in (5.50) one ends up with (6.5). This result is consistent with the scaling argument given in [CoGi93] which relates the viscosity to the product of the longest relaxation time $t^{*}$ and the modulus on that time scale $G\left(t^{*}\right) \sim\left(t^{*}\right)^{-\Delta}$. This argument follows from dimensional analysis and gives $\eta \sim\left(t^{*}\right)^{1-\Delta}$. It is not surprising that the fundamental scaling relation $k=z(1-\Delta)$ of critical rheology is recovered (cf. Sec. 1.3.1) since the scaling (5.48) of the shear relaxation function was assumed $a$ priori.

\subsubsection{First normal stress coefficient}

According to (5.8) the disorder averaged first normal stress coefficient (4.41) is given by

$$
\Psi^{(1)}=\sum_{i=1}^{\infty} n \tau_{n} \Psi_{n}^{(1)}
$$

with the partial average at the critical point

$$
\Psi_{n}^{(1)}:=\left.\left\langle\Psi^{(1)}(\cdot)\right\rangle_{n}\right|_{\varepsilon=0} .
$$

In order to compute the partial average (6.11) the first normal stress coefficient of a cluster $\mathscr{N}_{k}$ the expression (4.42) is written in the form

$$
\Psi^{(1)}\left(\mathscr{N}_{k}\right):=\frac{1}{k_{\mathrm{B}} T}\left(\frac{a^{2}}{3}\right)^{2} \frac{1}{2 N_{k}} \sum_{i, j \in \mathscr{N}_{k}}\left[\frac{1-E_{0}}{\Gamma}\right]_{i, j}\left[\frac{1-E_{0}}{\Gamma}\right]_{i, j} .
$$

From Eq. (6.12) it is plausible that a straightforward application of the derived scaling relation for the resistances $(5.21)$ is not as simple. This can be seen by inserting the expression (5.16) for the matrix element $\left[\left(\mathbb{1}-E_{0}\right) / \Gamma\right]_{i, j}$ in terms of the resistances into $(6.12)$ and subsequently into (6.11): It requires the computation of averages of the form $\left\langle\mathscr{R}_{i, j} \mathscr{R}_{k, l}\right\rangle_{n}$ for arbitrary nodes $i, j, k, l$ in the same cluster. In other words, it requires the joint probability 
of two resistances $\mathscr{R}_{i, j}, \mathscr{R}_{k, l}$ or equivalently by the conditional probability $\operatorname{Prob}\left(\mathscr{R}_{i, j} \mid \mathscr{R}_{k, l}\right)$ of $\mathscr{R}_{i, j}$ under the condition of fixed $\mathscr{R}_{k, l}$. Note, that fixing $\mathscr{R}_{k, l}$ imposes a constraint on the geometry of the cluster. However, for large cluster sizes $n \rightarrow \infty$ which give the dominant contributions to the critical behavior of $(6.10)$ this constraint is expected to be negligible, which means that the finite set of vertices $i, j, k, l$ in the nearly infinite cluster have independent environments. Therefore it seems plausible that the average factorizes for $n \rightarrow \infty$. Then, for $i \neq k$ or $j \neq l$ one has $\left\langle\mathscr{R}_{i, j} \mathscr{R}_{k, l}\right\rangle_{n} \sim\left\langle\mathscr{R}_{i, j}\right\rangle_{n}^{2}$, whereas for $i=k$ and $j=l$ one has $\left\langle\mathscr{R}_{i, j} \mathscr{R}_{k, l}\right\rangle_{n}=\left\langle\mathscr{R}_{i, j}^{2}\right\rangle_{n}$. Since for both ensembles, Erdős-Rényi random trees (5.26) and bond percolation (5.26) the square of the average resistance and the average of the squared resistance have the same scaling for $n \rightarrow \infty$, viz

$$
\left.\left.\left\langle\mathscr{R}_{i, j}\right\rangle_{n}^{2}\right|_{\varepsilon=0} \sim\left\langle\mathscr{R}_{i, j}^{2}\right\rangle_{n}\right|_{\varepsilon=0} \sim n \rho_{n}^{(2)},
$$

the aforementioned argument leads to the conjecture

$$
\Psi_{n}^{(1)} \sim n \rho_{n}^{(2)} \sim n^{4 / d_{\mathrm{s}}-1}
$$

for the scaling of the averaged first normal stress coefficient over clusters of size $n$. By inserting (6.14) into (6.10) and comparing with (5.13) the critical behavior

$$
\Psi^{(1)} \sim \varepsilon^{-\ell}, \quad \text { with } \quad \ell=\left(4 / d_{\mathrm{s}}+1-\tau\right) / \sigma
$$

is derived for the first normal stress coefficient.

This result is consistent with Erdős-Rényi random graphs where the normal stress coefficient can be computed from the second inverse moment $M_{2}$ (see Eq. (5.55)) of the density of states [BrLo01b]. It yields

$$
\Psi^{(1)} \sim M_{2}=c\left[-\frac{8 c^{3}-6 c^{2}-5 c+1}{30 c(1-2 c)^{3}}-\frac{4 c^{2}-3 c-1}{24 c(1-2 c)^{2}}+\frac{1}{240 c^{2}} \ln (1-2 c)\right]
$$

and exhibits a divergence $\Psi^{(1)} \sim \varepsilon^{-3}$ for $c \uparrow c_{\text {crit }}$ which is accordance with (6.15) for the mean field values $d_{\mathrm{s}}=4 / 3, \tau=5 / 2, \sigma=1 / 2$.

In fact, the conjecture $(6.15)$ is also confirmed by computing the first normal stress coefficient directly from the shear relaxation function $\Psi^{(1)} \sim \int_{0}^{\infty} \mathrm{d} t t G(t)$ with the scaling form (5.48) as done in [Mue03]. This gives the scaling relation $\ell=z(2-\Delta)$ and by virtue of the definitions (5.50) of $z, \Delta$ one arrives at (6.15). The numerical value is given by $\ell \approx 4.9$ for $d=3$ and $\ell=3$ for mean field percolation.

\subsubsection{Recoverable compliance}

In contrast to the previous examples, the average of the recoverable compliance cannot be computed directly, since the expression (4.43) cannot be written as a sum over contributions from individual clusters. However, since self-averaging is anticipated for all observables, the recoverable compliance (4.43) must be determined by the critical behavior of the viscosity (6.5) and the normal stress coefficient (6.15) according to

$$
J=\frac{\Psi^{(1)}}{2 \eta^{2}} \sim \frac{\varepsilon^{-\ell}}{\varepsilon^{-2 k}} \sim \varepsilon^{-(\ell-2 k)} .
$$


Note that $\ell-2 k=z \Delta$ which is in accordance with the following simple scaling picture. The recoverable compliance $J$ is an inverse elastic modulus on time scales $t \approx t^{*}$ where the liquid, macromolecular system still exhibits elastic behavior. After the time $t^{*}$ or for frequencies $\omega<\omega^{*}=1 / t^{*}$ the response of the system is fluid-like for all frequencies. The modulus on the terminal time scale is given by $G\left(t^{*}\right) \sim\left(t^{*}\right)^{-\Delta}$ which is a consequence of the scaling form (5.48). This implies $J \sim\left(G\left(t^{*}\right)\right)^{-1} \sim \varepsilon^{-z \Delta}$, in accordance with the fundamental scaling relation of critical rheology $\mu=z \Delta$ for the exponent $\mu$ of the shear modulus (cf. Sec. 1.3.1).

Denoting by $\theta$ the critical exponent of $J$ it can be expressed solely in terms of the exponents of the cluster size distribution

$$
J \sim \varepsilon^{-\theta}, \quad \text { with } \quad \theta=\frac{\tau-1}{\sigma}
$$

and is independent of $d_{\mathrm{s}}$. The numerical values are given by $\theta=2.62$ in $d=3$ and $\theta=3$ in $d=6$.

\subsubsection{Incoherent Scattering function}

Next, density fluctuations in the Rouse model are considered. The simplest quantity, the diffusion constant of a cluster of size $n$, is rather uninteresting in the Rouse model. According to (4.46) the diffusion constant of a cluster of size $n$ in the Rouse model is simply given by inverse number of monomers in the cluster [BrGo97]

$$
D_{n}=\frac{k_{\mathrm{B}} T}{n \zeta} .
$$

This is a consequence of the fact that the Rouse model assigns the same frictional force to each monomer in the cluster.

Scaling of the incoherent scattering function The average of the incoherent scattering function (4.50) is given by

$$
S(\boldsymbol{q}, t):=\langle S(\boldsymbol{q}, t \mid \cdot)\rangle=\sum_{n=1}^{\infty} n \tau_{n} S_{n}(\boldsymbol{q}, t)
$$

with the partial average

$$
S_{n}(\boldsymbol{q}, t):=\left.\left\langle\frac{1}{N_{k}} \sum_{i \in \mathscr{N}_{k}} \mathrm{e}^{-\frac{q^{2}}{2} D_{i, i}(t \mid \cdot)}\right\rangle\right|_{n} .
$$

The average $\langle\bullet\rangle_{n}$ of the exponential in (6.21) cannot be computed analytically. However, a lower bound to the scattering function can be derived by applying the Jensen inequality (cf. App. F) in the form $\langle\exp (\bullet)\rangle_{n} \geq \exp \left(\langle\bullet\rangle_{n}\right)$ to $(6.21)$. This yields

$$
S_{n}(\boldsymbol{q}, t) \geq \mathrm{e}^{-q^{2} \frac{1}{2 n} \operatorname{Tr} D_{n}(t)},
$$


where the average $D_{n}(t):=\left.\langle D(t \mid \cdot)\rangle_{n}\right|_{\varepsilon=0}$ of the matrix (4.27) of second moments of the increments has been defined. The bound can be related to the return probability $(5.44)$ of the blind ants random walk (5.43) via

$$
\frac{1}{2 n} \operatorname{Tr} D_{n}(t)=\frac{k_{\mathrm{B}} T}{\zeta} \int_{0}^{t} \mathrm{~d} t^{\prime} P_{n}\left(18 t^{\prime} / t_{\text {mic }}\right),
$$

and hence

$$
S(\boldsymbol{q}, t) \geq \sum_{n=1}^{\infty} n \tau_{n} \mathrm{e}^{-\left(k_{\mathrm{B}} T / \zeta\right) q^{2} \int_{0}^{t} P_{n}\left(18 t / t_{\mathrm{mic}}\right)}
$$

If $18 t \gg t_{\text {mic }}$ the scaling form (5.44) can be inserted into (6.24). Therefore, it is convenient to introduce a critical, characteristic length scale, which is termed Gaussian correlation length

$$
\xi^{*}:=a \varepsilon^{-\nu^{(\mathrm{G})}}
$$

with the Gaussian correlation length exponent

$$
\nu^{(\mathrm{G})}:=1 /\left(\sigma d_{\mathrm{f}}^{(\mathrm{G})}\right)=\left(1 / d_{\mathrm{s}}-1 / 2\right) / \sigma .
$$

The Gaussian correlation length exponent $(6.26)$ is defined in analogy to lattice percolation where the correlation length exponent $\nu$ is related to the Hausdorff fractal dimension $d_{\mathrm{f}}^{(\mathrm{H})}$ of lattice percolation clusters in the same way $\nu=1 /\left(\sigma d_{\mathrm{f}}^{(\mathrm{H})}\right)$ (see Tab. 1.1). The Gaussian correlation length is thus proportional to the radius of gyration of the largest cluster with size $n^{*}$. By inserting the scaling behavior (5.44) of $P_{n}(t)$ and the cluster size distribution $\tau_{n}=n^{-\tau} f\left(n / n^{*}\right)$ into (6.24) the bound can be written in terms of the scaling variables $t / t^{*}, q \xi^{*}, n / n^{*}$

$$
\begin{aligned}
& S(\boldsymbol{q}, t) \geq \int_{0}^{\infty} \mathrm{d} n n^{1-\tau} f\left(-n / n^{*}\right) \\
& \exp \left\{-\left(q \xi^{*}\right)^{2}\left(t / t^{*}\right)\left(n^{*} / n\right)[1+\right. \\
&\left.\left.+\frac{\left(n / n^{*}\right)}{\left(t / t^{*}\right)^{d_{\mathrm{s}} / 2}} \int_{0}^{1} \mathrm{~d} y y^{-d_{\mathrm{s}} / 2} \mathcal{F}_{P}\left(y \frac{\left(t / t^{*}\right)}{\left(n / n^{*}\right)^{2 / d_{\mathrm{s}}}}\right)\right]\right\} .
\end{aligned}
$$

It is suggested that this bound correctly recovers the scaling properties of the incoherent scattering function. However, it has not been possible to show this analytically. The accuracy of the bond relies on the accuracy of the Jensen inequality in (6.22). One may suggest that the bound amounts to an equality for the following reason: In [Loe99, BrLo01a] it was shown for Erdôs-Rényi random graphs that the application of the Jensen inequality in a similar case does not affect the asymptotic long time behavior of the scattering function for $t \gg t^{*}$. 
Long time decay of the incoherent scattering function Next, the disorder averaged upper bound (4.54) of the incoherent scattering function is examined. With the expression (6.19) for the diffusion constant the upper bound

$$
S(\boldsymbol{q}, t) \leq \sum_{n=1}^{\infty} n \tau_{n} \mathrm{e}^{-D_{n} q^{2} t}
$$

is obtained [Loe99, BrLo01a], which has a striking physical interpretation. On the longest time scales $t \gg t^{*}$ only the diffusive motion of the clusters contribute to the decay of the scattering function. Possible dynamical processes stemming from the relaxation of internal modes have already decayed. Expression (6.28) has also been proposed by de Gennes on phenomenological grounds [Gen79a]. For Erdős-Rényi random graphs it can be proved (see [Loe99, BrLo01a]) that the long time decay of the bound (6.28) coincides with the long time decay of the scattering function. The proof requires the precise knowledge of the cluster size distribution which is exactly known only for Erdős-Rényi random trees. If, however, (6.28) is also expected as the true long time behavior for bond percolation, then the long time behavior of both ensembles can be combined in the same functional scaling form. Therefore, the sum $(6.28)$ is converted into an integral for $\varepsilon \ll 1$. This yields (see [BrLo01a] or App. E.2)

$$
S(\boldsymbol{q}, t) \sim\left(q^{2} t\right)^{\delta} \mathcal{F}_{S}\left(t / t_{q}\right),
$$

with a $q$-dependent, diffusive, diverging time scale

$$
t_{q} \sim q^{-2} \varepsilon^{-\tilde{z}}, \quad \text { with } \quad \tilde{z}=1 / \sigma .
$$

The scaling function $\mathcal{F}_{S}(x)$ is of order unity for $x \rightarrow 0$ and decays like a stretched exponential $\mathcal{F}_{S}(x) \sim x^{\alpha_{S}(\delta-1 / 2)} \exp \left\{-c x^{\alpha_{S}}\right\}$ for $x \rightarrow \infty$. Neglecting algebraic prefactors, the incoherent scattering function decays according to a stretched exponential

$$
S(\boldsymbol{q}, t) \sim \exp \left\{-\left(t / t_{q}\right)^{\alpha_{S}}\right\}, \quad \text { with } \quad \alpha_{S}=1 / 2
$$

in the sol phase and according to a power law

$$
S(\boldsymbol{q}, t) \sim \frac{1}{\left(q^{2} t\right)^{\delta}}, \quad \text { with } \quad \delta=\tau-2
$$

at the critical point. The exponent of the stretched exponential does not depend on the cluster statistics in the Rouse model. This is similar to the stretched exponential decay of the shear relaxation function $G(t)$ where the exponent $\alpha_{G}=1 / 3$ is valid also for both crosslink ensembles (see below Eq. 5.57).

Next, the effective diffusion constant (3.29) is computed, which is given by the time integral over the scattering function. Likewise, one can integrate the lower bound (6.28) which presumably has the same long time decay which dominates the integral in the vicinity of the transition. This implies

$$
D_{\text {eff }}^{-1}=\sum_{n=1}^{\infty} n \tau_{n} D_{n}^{-1} .
$$


Using $D_{n} \sim 1 / n$ and the formula (5.13) Eq. (6.33) gives rise to the divergence [BrLo01a]

$$
D_{\text {eff }}^{-1} \sim \varepsilon^{-u}, \quad \text { with } \quad u=(3-\tau) / \sigma
$$

Expression (6.33) reveals the significance of the effective diffusion constant: It is the diffusion constant of a cluster with weight-averaged cluster size $n_{\mathrm{w}} \sim \varepsilon^{-\gamma}$ (cf. Eq. (1.4))

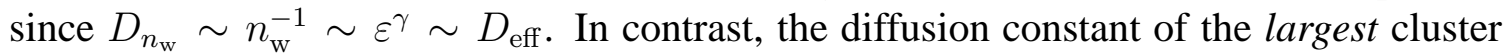
$D_{n^{*}} \sim \varepsilon^{1 / \sigma}$ vanishes more rapidly.

One may be confused that the discussion of density fluctuations apparently introduces a new time scale $t_{q}$ with a critical divergence being different from that of $t^{*}$. The time scale $t^{*}$ is the longest time scale in the system and can be related to the diffusive time scale of those clusters having size $n^{*}$ and spatial extension $\xi^{*}$, viz $t^{*}=\left(\xi^{*}\right)^{2} / D_{n^{*}}$. Moreover, one would expect that $t^{*}$ is the only relevant time scale and $\xi^{*}$ the only relevant length scale in the vicinity of the critical point. Hence, dimensional analysis dictates the diffusive time scale $t_{q} \sim q^{-2}$ being related to the fundamental scales according to

$$
t_{q}=\frac{t^{*}}{\left(q \xi^{*}\right)^{2}} .
$$

Equation (6.35) then predicts $t_{q} \sim \varepsilon^{2 \nu^{(\mathrm{G})}-z}$ which coincides with $(6.30)$ by virtue of the definitions of $\nu^{(\mathrm{G})}$ and $z$ in Eqs. (6.26) and (5.50), respectively. This implies the scaling relation

$$
\tilde{z}=z-2 \nu^{(\mathrm{G})}
$$

Time persistent contribution of the incoherent scattering function Finally, the limit $t \rightarrow \infty$ of the incoherent scattering function $(6.20)$ is considered. The time persistent contribution

$$
S_{\infty}(\boldsymbol{q}):=\lim _{t \rightarrow \infty} S(\boldsymbol{q}, t)
$$

for $c>c_{\text {crit }}$ plays the role of the order parameter for the gelation transition in the Rouse model. For that purpose, the long time limit of the matrix of displacements

$$
D_{i, i}(t \mid \mathscr{G})=\frac{2 k_{\mathrm{B}} T}{\zeta}\left(\frac{1}{N(i)} t+\int_{0}^{t} \mathrm{~d} t^{\prime}\left[\left(\mathbb{1}-E_{0}\right) \exp \left\{-3 \Gamma(\mathscr{G}) t^{\prime} / t_{\mathrm{mic}}\right\}\right]_{i, i}\right)
$$

is examined more precisely. Whenever the number of monomers $N(i)$ of the cluster of monomer $i$ remains finite in the macroscopic limit $D_{i, i}(t \mid \mathscr{G})$ tends to infinity for $t \rightarrow \infty$ reflecting an unbounded mean squared displacement due to diffusive motion of the center of mass. On the other hand, if $i$ belongs to the incipient macroscopic cluster then $N(i)$ tends to infinity which suppresses the diffusive motion of that cluster. This corresponds to a localization of the incipient infinite cluster $\mathscr{N}_{\infty}$ in space. In fact, this result relies on the order of the limits, the long time limit is to be taken after the macroscopic limit.

As demonstrated in App. C.1 the long time limit is given by

$$
\lim _{t \rightarrow \infty} D_{i, i}(t \mid \mathscr{G})= \begin{cases}\infty & i \notin \mathscr{N}_{\infty} \\ \frac{2 a^{2}}{3}\left[\frac{\mathbb{1}-E_{0}}{\Gamma}\right]_{i, i} & i \in \mathscr{N}_{\infty}\end{cases}
$$


which implies

$$
S_{\infty}(\boldsymbol{q}):=\lim _{t \rightarrow \infty} S(\boldsymbol{q}, t)=\left\langle\frac{1}{N} \sum_{i \in \mathscr{N}_{\infty}} \exp \left\{-q^{2} a^{2}\left[\frac{\mathbb{1}-E_{0}}{\Gamma}\right]_{i, i}\right\}\right\rangle
$$

for the scattering function (6.20).

Thus, the time persistent part of the incoherent scattering function is nothing but the characteristic function (5.36) of the resistance to infinity, viz $S_{\infty}(\boldsymbol{q})=Z_{\infty}(|\boldsymbol{q}|)$. It can be written as

$$
S_{\infty}(\boldsymbol{q})=S_{\infty} \int_{0}^{\infty} \mathrm{d} x \pi(x) \mathrm{e}^{-\left(q \xi^{*}\right)^{2} / x}
$$

in terms of the gel fraction $S_{\infty}$ according to Eq. (5.37). This is interpreted as follows: $\pi(x)$ plays the role of a distribution of squared, inverse localization lengths which characterizes the thermal excursions of a particle in the macroscopic cluster. The length scale of these excursions is set by $\xi^{*}$. The expression (6.41) is formally identical to the result (3.32) which has been derived within a mean field approximation of the gelation transition in $d=6$ in the presence of excluded volume interactions. The difference between both expressions lies in the associated length scales. In the presence of excluded volume interactions the order parameter (3.32) involves the correlation length of lattice percolation $\xi$ whereas in the absence of excluded volume interactions (5.37) involves the Gaussian correlation length $\xi^{*}$.

However, it is emphasized that the result (6.41) bears a serious conceptual problem. It is well known that excluded volume interactions are indispensable in the gel phase for $c \geq c_{\text {crit }}$ in order to guarantee a stable equilibrium phase [BaEd80]. Without repulsive interactions it is not possible to maintain a homogeneous system with a constant monomer density. This is plausible, since in the absence of excluded volume interactions cluster radii are governed by the Gaussian fractal dimension which is larger than the spatial dimension. Thus, the monomer density $n /\left(R_{n}\right)^{d} \sim n^{1-d / d_{\mathrm{f}}^{(\mathrm{G})}}$ within a cluster of size $n$ exhibits a singularity for the incipient macroscopic cluster for $n \rightarrow \infty$. This prevents homogeneity. Nevertheless, one might imagine that a gelling system is governed by a Gaussian fractal dimension up to a particular length scale and thus (5.37) might be valid at least on certain length scales even for for $c \geq c c$. But, unless it is possible to identify the phantom cluster description of percolation clusters with a meaningful limit of a realistic system, the result (6.41) remains a formal one. This point is again taken up in the discussions.

\subsubsection{Order parameter susceptibility}

The disorder average of the bound to the nonlinear dynamic susceptibility (4.59) is given by

$$
\chi(\boldsymbol{q}, t) \geq\left\langle\frac{1}{N} \sum_{i, j}^{N} \mathrm{e}^{-\frac{q^{2}}{2}\left[D_{i, i}(t)+D_{j, j}(t)-2 D_{i, j}(t)\right]}\right\rangle .
$$


According to $(5.12)$ this can be written in the form

$$
\chi(\boldsymbol{q}, t) \geq \sum_{n=1}^{\infty} n^{2} \tau_{n}\left\langle\frac{1}{N_{k}^{2}} \sum_{i, j \in \mathscr{N}_{k}} \mathrm{e}^{-\frac{q^{2}}{2}\left[\left(D_{i, i}(t)+D_{j, j}(t)-2 D_{i, j}(t)\right]\right\rangle_{n} .}\right.
$$

Similar to the scattering function the average of the exponential in (6.43) cannot be performed exactly. Therefore, the Jensen inequality is applied to (6.43) by shifting both averages, $\langle\bullet\rangle_{n}$ and $N_{k}^{-2} \sum_{i, j}(\bullet)$ into the argument of the exponential. From Eq. (C.5) the equality $N_{k}^{-2} \sum_{i, j \in \mathscr{N}_{k}} D_{i, j}\left(t \mid \mathscr{N}_{k}\right)=2 k_{\mathrm{B}} T t /\left(\zeta N_{k}\right)$ is inferred and by setting $H=\mathbb{1}$ the lower bound

$$
\begin{aligned}
\chi(\boldsymbol{q}, t) & \geq \sum_{n=1}^{\infty} n^{2} \tau_{n} \exp \left\{-2 q^{2}\left(\frac{1}{2 n} \operatorname{Tr} D_{n}(t)-\frac{k_{\mathrm{B}} T t}{\zeta n}\right)\right\} \\
& =\sum_{n=1}^{\infty} n^{2} \tau_{n} \exp \left\{-\frac{2 k_{\mathrm{B}} T}{\zeta} q^{2} \int_{0}^{t} \mathrm{~d} t^{\prime}\left(P_{n}\left(18 t^{\prime} / t_{\text {mic }}\right)-1 / n\right)\right\}
\end{aligned}
$$

is obtained. Here, the second equation is obtained by inserting (6.23). By assuming $t \gg$ $t_{\text {mic }}$ the scaling form (5.44) of the return probability $P_{n}(t)$ can be inserted. In the vicinity of the critical point the sum over clusters is converted into an integral and by substituting $x:=n / n^{*}=n \varepsilon^{1 / \sigma}$ and $y:=t^{\prime} / t$ one gets

$$
\begin{aligned}
& \chi(\boldsymbol{q}, t) \gtrsim \varepsilon^{-(3-\tau) / \sigma} \int_{\varepsilon^{1 / \sigma}}^{\infty} \mathrm{d} x x^{2-\tau} f(x) \\
& \exp \left\{-\frac{2 k_{\mathrm{B}} T}{\zeta} q^{2}\left(t / t_{\mathrm{mic}}\right)^{1-d_{\mathrm{s}} / 2} \int_{0}^{1} \mathrm{~d} y y^{-d_{\mathrm{s}} / 2} \mathcal{F}_{P}\left(\frac{\left.y t \varepsilon^{2 /\left(\sigma d_{\mathrm{s}}\right.}\right)}{x^{2 / d_{\mathrm{s}}}}\right)\right\} .
\end{aligned}
$$

With the time scale $t^{*}=t_{\text {mic }} \varepsilon^{-z}$ from (5.49) and the length scale $\xi^{*}$ from (6.25) it is readily verified that $\left(k_{\mathrm{B}} T / \zeta\right) q^{2} t^{1-d_{\mathrm{s}} / 2}=\left(q \xi^{*}\right)^{2}\left(t / t^{*}\right)^{1-d_{\mathrm{s}} / 2} t_{\text {mic }}^{-d_{\mathrm{s}} / 2}$. In the limit $\varepsilon \downarrow 0$ the integral has a well defined limit for $\tau<3$ and hence the scaling relation

$$
\chi(\boldsymbol{q}, t) \gtrsim \varepsilon^{-\gamma} \mathcal{F}_{\chi}\left(q \xi^{*}, t / t^{*}\right), \quad \text { for } \varepsilon \downarrow 0
$$

is derived for the bound of the nonlinear susceptibility with a scaling function

$$
\mathcal{F}_{\chi}\left(z_{1}, z_{2}\right)=\int_{0}^{\infty} \mathrm{d} x x^{2-\tau} f(x) \exp \left\{-2 z_{1} z_{2}^{1-d_{\mathrm{s}} / 2} \int_{0}^{1} \mathrm{~d} y y^{-d_{\mathrm{s}} / 2} \mathcal{F}_{P}\left(z_{2} y / x^{2 / d_{\mathrm{s}}}\right)\right\} .
$$

The scaling form (6.47) which has been derived for the bound to the nonlinear dynamic susceptibility is of exactly the same form one would anticipate within the dynamic scaling assumption [HoHa77, Sec. III C.2]. Unfortunately, it is not possible to prove the scaling behavior also for $(6.42)$. Nonetheless, the result is reasonable and demonstrates the relevance of the scales $\xi^{*}$ and $t^{*}$ in the Rouse model. 


\subsection{Zimm model}

Observables in the Zimm model are obtained from the formulas derived in Secs. 4.2 and 4.3 with $H \neq \mathbb{1}$.

\subsubsection{Shear viscosity and first normal stress coefficient}

The disorder averages of the viscosity (4.37) and the first normal stress coefficient (4.41) in the Zimm model are determined by the scaling behaviors

$$
\begin{aligned}
\eta_{n} & =\left.\langle\eta(\cdot)\rangle_{n}\right|_{\varepsilon=0} \sim n^{b_{\eta}} \\
\Psi_{n}^{(1)} & =\left.\left\langle\Psi^{(1)}(\cdot)\right\rangle_{n}\right|_{\varepsilon=0} \sim n^{b_{\Psi}}
\end{aligned}
$$

via the familiar relations

$$
\eta=\sum_{n=1}^{\infty} n \tau_{n} \eta_{n}, \quad \Psi^{(1)}=\sum_{n=1}^{\infty} n \tau_{n} \Psi_{n}^{(1)} .
$$

Note, that the viscosity (4.38) and the first normal stress coefficient (4.42) are now determined by the matrix $\widetilde{\Gamma}=H^{\frac{1}{2}} \Gamma H^{\frac{1}{2}}$ where the matrices $\Gamma$ and $H$ are either computed for Erdős-Rényi random graphs or for bond percolation as described in Sec. 5.5.1, 5.5.2, respectively. As already mentioned, $\widetilde{\Gamma}=H^{\frac{1}{2}} \Gamma H^{\frac{1}{2}}$ and $\widehat{\Gamma}=H \Gamma$ have the same eigenvalues (this is proved in App. B.2). For numerical purposes it is advantageous to use $\widehat{\Gamma}$ instead of $\widetilde{\Gamma}$ in order to avoid the computation of the square root $H^{\frac{1}{2}}$. For each generated cluster the product $\widehat{\Gamma}:=H \Gamma$ is computed and numerically diagonalized by the LAPACK routines dgehrd_ and dhsegr_ . The smallest eigenvalue of $\widehat{\Gamma}$, which is a nonzero number in computer precision is regarded as the null eigenvalue. The viscosity is given by the sum over the inverse, nonzero eigenvalues and the normal stress coefficient by the sum over the squares of inverse, nonzero eigenvalues.

\subsubsection{Numerical results}

Erdôs-Rényi random graphs For Erdôs-Rényi random graphs suitable, logarithmically equidistant cluster sizes $n \in[2,4000]$ are chosen, in order to reduce the computational effort. For each cluster size the viscosity and the first normal stress coefficient are averaged over 50 trees, which turned out to yield an acceptable computer-time/accuracy trade-off. In Figs. 6.1(a) and (b) $\eta_{n}$ and $\Psi_{n}^{(1)}$ are plotted as a function of $n$ on a double-logarithmic scale for different values of the hydrodynamic interaction parameter $\kappa$. According to (6.49) the exponents $b_{\eta}$ and $b_{\Psi}$ are obtained by power law fits in the large $n$-range, for which the interval $n \in[700,4000]$ is chosen, see Fig. 6.1(c). For the viscosity the exponent decreases from $b_{\eta}=0.28$ for $\kappa=0.05$ to $b_{\eta}=0.11$ for $\kappa=0.3$. The Rouse exponent for $\kappa=0$ is exactly given by $b_{\eta}=1 / 2$ (cf. Eq. (6.4)). The exponent $b_{\Psi}$ of the normal stress coefficient ranges from $b_{\Psi}=1.2$ for $\kappa=0.05$ to $b_{\Psi}=0.73$ for $\kappa=0.25$. The Rouse value for $\kappa=0$ is exactly given by $b_{\Psi}=2$ (cf. Eq. (6.14)). 

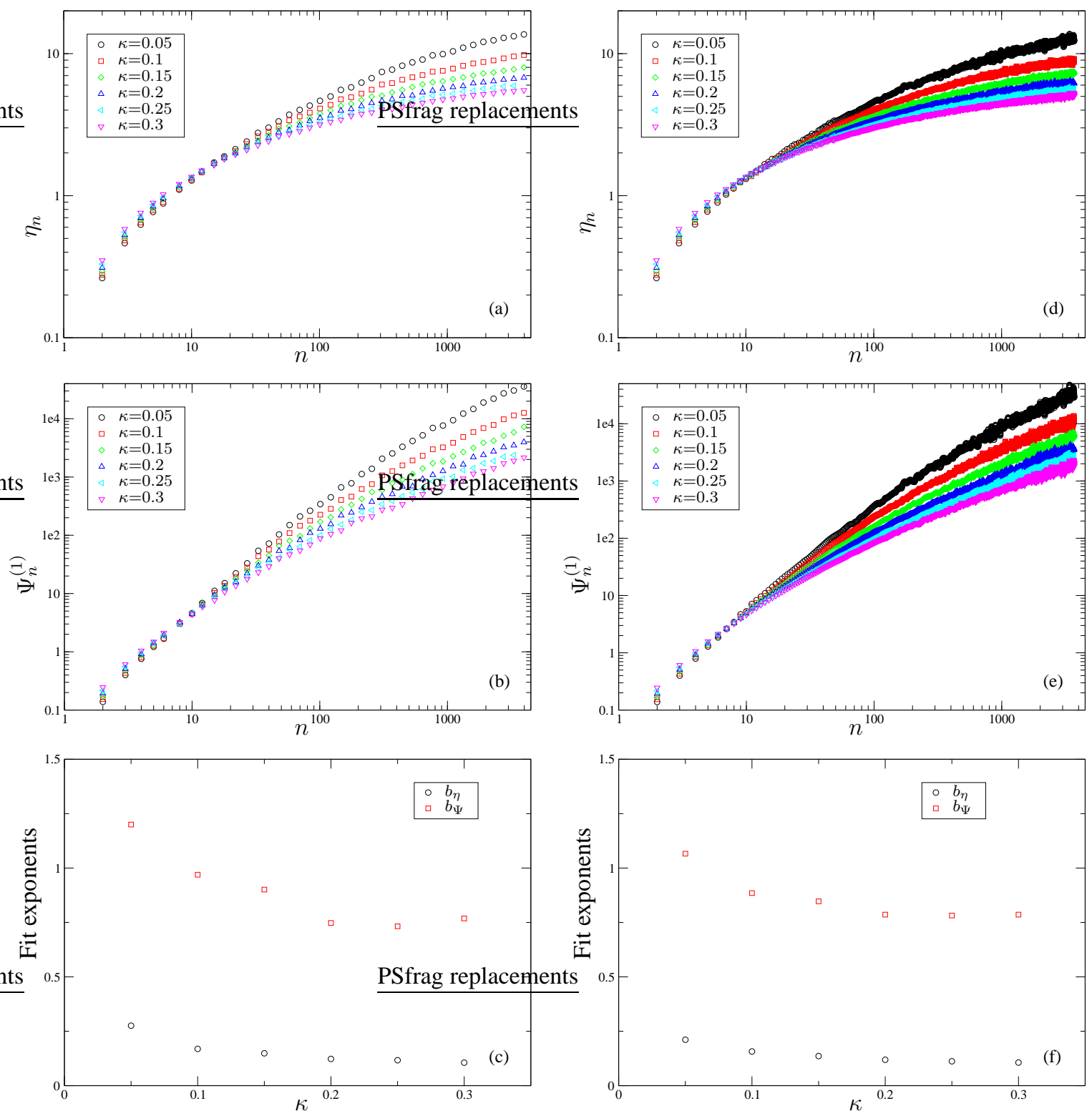

Figure 6.1: Numerical data to determine the scaling (6.49) for random clusters in the case of Erdős-Rényi random graphs (left column) and three-dimensional bond percolation (right column). In each case the averaged viscosity $\eta_{n}$ (top) and normal stress coeffi cient $\Psi_{n}^{(1)}$ (middle) are plotted for different strengths of the hydrodynamic interaction parameter $\kappa$ as a function of the cluster size $n$ on a double logarithmic scale. Power-law fi ts to the data yield the exponents $b_{\eta}$ and $b_{\Psi}$ as a function of $\kappa$ (bottom). 

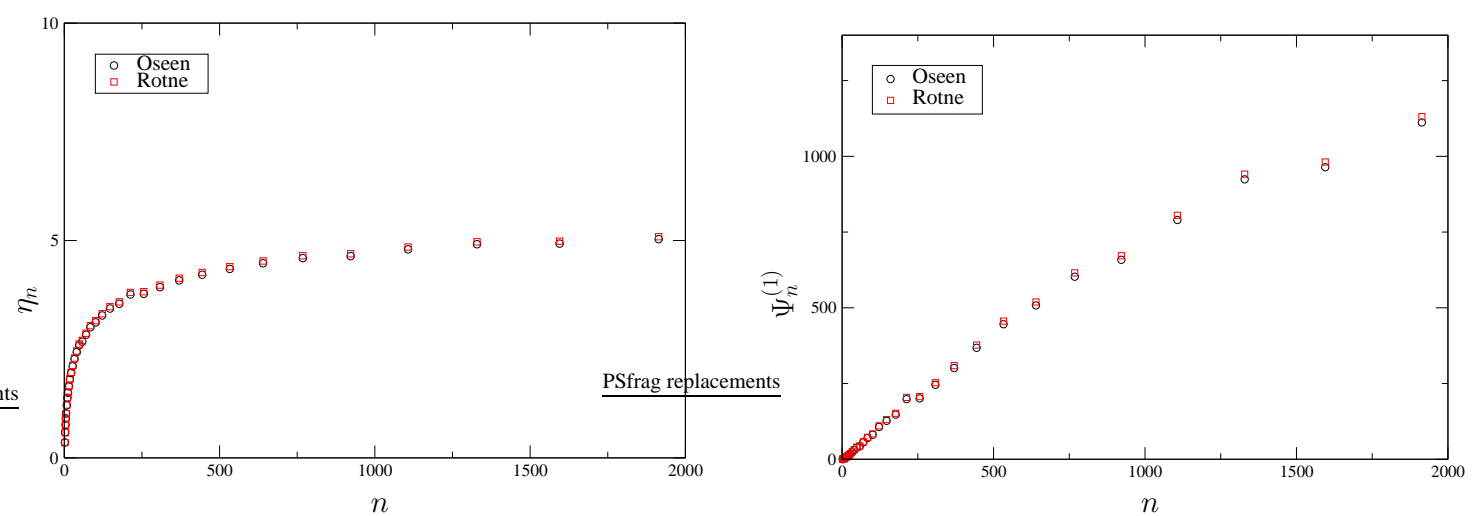

Figure 6.2: Comparison between Rotne-Prager and the Oseen tensor. The viscosity (left) and the fi rst normal stress coeffi cient (right) for Erø̆s-Rényi random graphs have been computed for $\kappa=0.3$. Note, that the axis-scale is linear, so that the differences between both are indeed negligible

The same results are obtained if the Oseen tensor is used instead of the Rotne-Prager tensor. This is demonstrated in Fig. 6.2 where the viscosity and the first normal stress coefficient computed from the Rotne-Prager tensor are compared with the Oseen tensor for cluster sizes $n \leq 1914$ for $\kappa=0.3$. The relative deviation for $n=1914$ is of order $1 \%$ for the viscosity and $2 \%$ for the normal stress coefficient and corresponding fits for the exponents give the same results within numerical accuracy.

Bond percolation The generation of clusters is restricted to values $n<4000$ due to the limited amount of memory, which is required for the generation and diagonalization of the matrix product $\widehat{\Gamma}=H \Gamma$. Moreover, for calculating disorder averages the number of realizations pertaining to a given cluster size is restricted to a maximum of 50 . However, within the present numerical effort this maximum number is not even attained for larger cluster sizes. Therefore the disorder averaged quantities are still subject to fluctuations. In order to obtain smooth curves for $\eta_{n}$ and $\Psi_{n}^{(1)}$ the raw data is smoothed out by performing a running average over cluster sizes in the window $[n-5, n+5]$. The thus obtained values for $\eta_{n}$ and $\Psi_{n}^{(1)}$ are plotted in Figs. 6.1(d) and (e), respectively, as a function of $n$ on a double-logarithmic scale for different values of $\kappa$. The exponents $b_{\eta}$ and $b_{\Psi}$, extracted by fitting the curves in Figs. 6.1(d) and (e) to a power law in the interval $n \in[800,4000]$, are shown in Fig. 6.1(f). The numerical values for $b_{\eta}$ are nearly identical to those obtained for Erdôs-Rényi random graphs. Again, one observes a decrease from $b_{\eta}=0.21$ for $\kappa=0.05$ to $b_{\eta}=0.11$ for $\kappa=0.3$. The exponent $b_{\Psi}$ of the normal stress coefficient ranges from $b_{\Psi}=1.1$ for $\kappa=0.05$ to $b_{\Psi}=0.78$ for $\kappa=0.25$. The corresponding Rouse values for $\kappa=0$ follow from Eq. (6.5) and (6.15).

For both Erdôs-Rényi random graphs and bond percolation similar results are obtained: The exponents $b_{\eta}, b_{\Psi}$ appear to depend on the strength of the hydrodynamic interaction 
parameter $\kappa$. The origin of the observed variation may be due to finite-size effects or possible non-universal behavior. The present numerical calculations are not capable of clarifying the origin of the observed variations with $\kappa$ neither for Erdôs-Rényi random graphs nor for bond percolation due to the limitations of available cluster sizes. In order to better understand finite-size effects and the onset of the asymptotic scaling regime, it is useful to study a system where the scaling exponents are known analytically. Therefore the viscosity $\eta_{\text {ring }}$ and the first normal stress coefficient $\Psi_{\text {ring }}^{(1)}$ of ring polymers in the Zimm model are re-investigated with the Rotne-Prager tensor.

\subsubsection{A reference system: Ring polymers}

For a ring polymer $\mathscr{C}_{n}$ of size $N$ the connectivity matrix $\Gamma\left(\mathscr{C}_{n}\right)$ differs from the connectivity matrix of the linear chain $(2.17)$ only by one bond

$$
\Gamma\left(\mathscr{C}_{n}\right)=\left(\begin{array}{rrrrr}
2 & -1 & 0 & \ldots & -1 \\
-1 & 2 & -1 & & \vdots \\
0 & & \ddots & & 0 \\
\vdots & & -1 & 2 & -1 \\
-1 & \ldots & 0 & -1 & 2
\end{array}\right)
$$

Due to the cyclic structure of the ring $\Gamma\left(\mathscr{C}_{n}\right)$ is a circulant matrix, i.e. the matrix elements $\Gamma_{i, j}\left(\mathscr{C}_{n}\right)$ depend only on $(|i-j| \bmod n)$. The pair resistances $\mathscr{R}_{i, j}$ have the same property and so has $H\left(\mathscr{C}_{n}\right)$. All circulant matrices are simultaneously diagonalizable. In fact, the $j$-th component of the $l$-th eigenvector of $\widehat{\Gamma}\left(\mathscr{C}_{n}\right)=H\left(\mathscr{C}_{n}\right) \Gamma\left(\mathscr{C}_{n}\right)$ for a ring of size $n$ is explicitly given by $\psi_{j}^{(l)}=\exp (\mathrm{i} 2 \pi j l / n)$. The eigenvalues $\widehat{\gamma}_{l}$ of $\widehat{\Gamma}\left(\mathscr{C}_{n}\right)$ can be written in a Fourier representation

$$
\widehat{\gamma}_{l}=2(1-\cos (2 \pi l / n)) \sum_{k=0}^{n-1} h_{k} \exp \left\{\mathrm{i} 2 \pi \frac{k l}{n}\right\}
$$

for $l=0,1, \ldots n-1$ where the coefficients $h_{k}$ are defined by $h_{k}:=h\left(\kappa^{2} \pi n /(k(n-k))\right)$. The eigenvalues are real valued which can be seen from $h_{k}=h_{n-k}$ for $k=1,2, \ldots n-1$ (cf. also [OeZy92]). The discrete Fourier transforms (6.53) can be computed efficiently by Fast Fourier Transformation up to ring sizes $n=10^{5}$ with the Numerical Recipes routine four1 . The resulting viscosity $\eta_{\text {ring }}$ and the first normal stress coefficient $\Psi_{\text {ring }}^{(1)}$ are shown in Figs. 6.3 (a) and (b) on a double logarithmic scale. The data is then fitted to a power law in two different fit ranges. In addition to a fit in the terminal large- $n$ range, $n \in\left[10^{4}, 10^{5}\right]$, a second fit in the range $n \in[500,5000]$ is performed, which is roughly where the fits in the random-cluster case are done. The fit exponents are shown in Fig. 6.3(c). Apparently, they depend on the fit range. For $\kappa=0.05$ an exponent $b_{\eta, \text { ring }}=0.69$ is found from the small- $n$ fit. This value clearly exceeds the theoretical one $b_{\eta \text {,ring }}=1 / 2$ which can be deduced from long-standing analytical results, [BlZi66] (see also App. $\mathrm{H}$ ). Even the corresponding value 


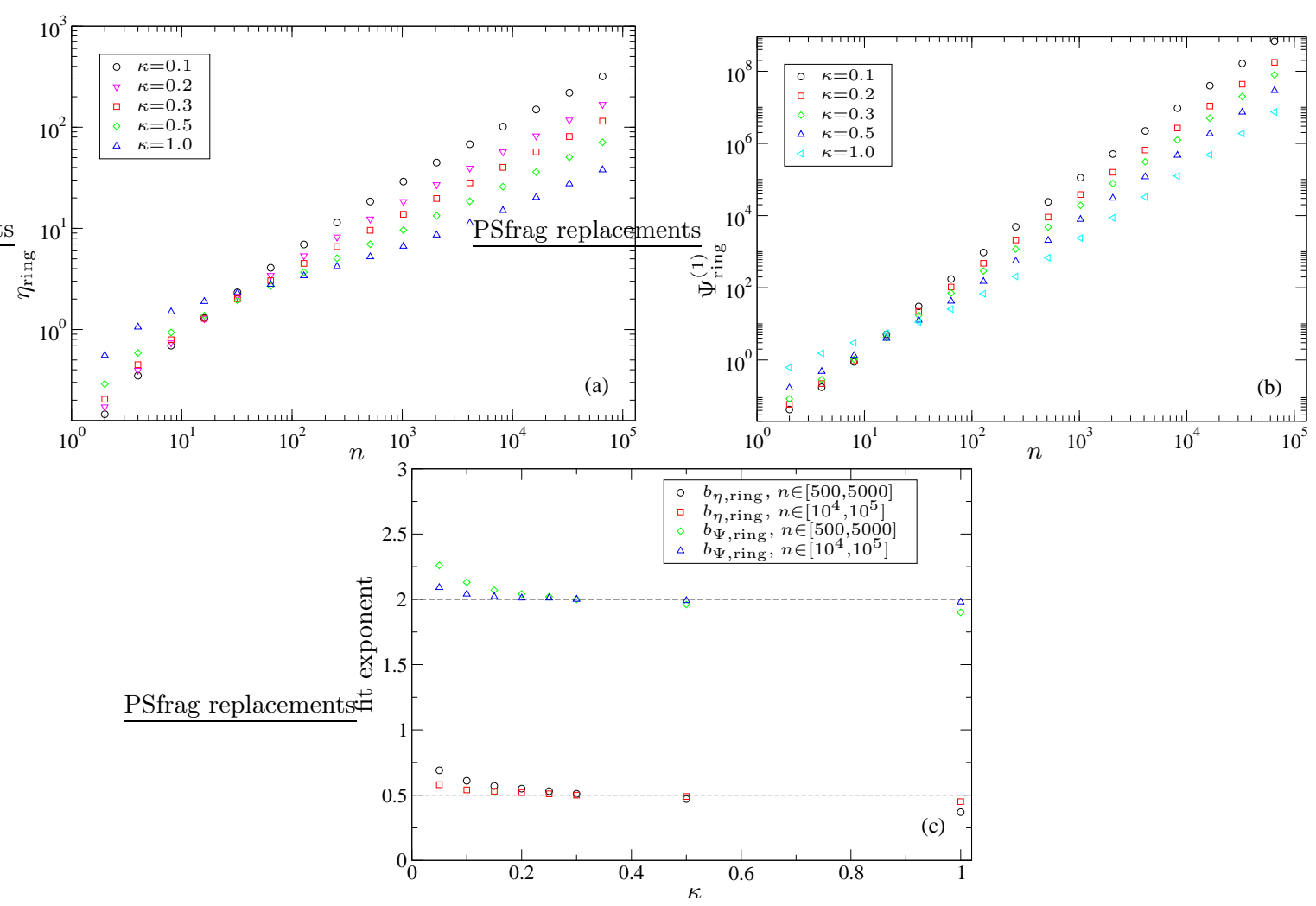

Figure 6.3: Numerical data to determine the scaling (6.49) for ring polymers. The viscosity $\eta_{\text {ring }}$ (a) and the normal stress coeffi cient $\Psi_{\text {ring }}^{(1)}$ (b) are plotted for different strengths of the hydrodynamic interaction parameter $\kappa$ as a function of the cluster size $n$ on a double logarithmic scale. (c) shows the exponents $b_{\eta, \text { ring }}$ and $b_{\Psi \text {,ring }}$ from power-law fi ts to the data of (a) and (b). The fi ts were performed for two different ranges of cluster sizes $n$. Additional data points for $\kappa=0.05,0.15$ and 0.25 in (c) stem from curves which have been omitted in (a) and (b) for reasons of clarity. The two horizontal lines indicate the exact values $b_{\eta, \text { ring }}=1 / 2$ and $b_{\Psi, \text { ring }}=2$.

$b_{\eta, \text { ring }}=0.58$ from the large- $n$ fit still has an error of $36 \%$. In contrast, for $\kappa=0.3$ both values, $b_{\eta, \text { ring }}=0.51$ and 0.50 , are quite close to the exact one. The same characteristic behavior holds true for $b_{\Psi \text {,ring }}$ with the exact value given by $b_{\Psi \text {,ring }}=2$ (see App. $\mathbf{H}$ ).

The observed variation of the exponents $b_{\eta, \text { ring }}$ and $b_{\Psi, \text { ring }}$ is quite similar to the random cluster case. However, for ring polymers it is straightforward to demonstrate (see App. $\mathrm{H}$ ) by means of the Fourier representation (6.53) the occurrence of a crossover at $\widehat{n} \approx \pi / \kappa^{2}$ from Rouse behavior, $\eta_{\text {ring }} \sim n$, to the asymptotic Zimm behavior $\eta_{\text {ring }} \sim n^{1 / 2} / \kappa$ for all $n \gg \kappa^{-2}$. Hence, the larger $\kappa$, the less important is residual Rouse behavior in the

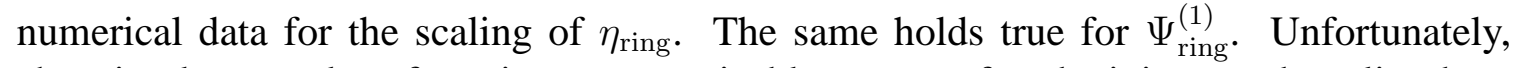
choosing larger values for $\kappa$ is not a practicable way out for obtaining good-quality data. 
This is because for large $\kappa$ the asymptotics

$$
h(x) \sim 1-(\pi x)^{-1 / 2}
$$

of $(2.31)$ as $x \rightarrow \infty$ becomes noticeable and leads to the transient behavior $\eta_{\text {ring }} \sim \kappa n^{0}$ for intermediate $n$ as shown in App. $\mathrm{H}$. This behavior is observed for (unphysically large) $\kappa>10$. But even the data for $\kappa=0.5$ and $\kappa=1.0$ in Fig. 6.3 is still slightly influenced by (6.54).

In summary, if the scaling with ring size $n$ of the viscosity $\eta_{\text {ring }} \sim n^{b_{\eta, \text { ring }}}$ and the first normal stress coefficient $\Psi_{\text {ring }}^{(1)} \sim n^{b_{\Psi, \text { ring }}}$ is considered one can access much higher values of $n$ as for random clusters, see Figs. 6.3(a) and (b). In particular, the exactly known scaling exponents $b_{\eta \text {,ring }}=1 / 2$ and $b_{\Psi \text {,ring }}=2$, which are universal in $\kappa>0$, can be extracted from the data in Fig. 6.3(c). However, if the full range of available ring sizes is not exploited and the fit is restricted to those lower values of $n$ which could also be accessed for random clusters, then universality would be veiled by finite-size effects. Finite-size effects are more pronounced for $\kappa \leq 0.15$ and $\kappa>0.5$.

\subsubsection{Critical behavior}

Taking into account the results obtained for ring polymers the following conclusions are drawn for the numerical data in the random cluster case: (i) The viscosity $\eta_{n}$ and the first normal stress coefficient $\Psi_{n}^{(1)}$ show a crossover from initial Rouse behavior for $\kappa=0$ to universal Zimm behavior for $\kappa>0$. (ii) The universal large- $n$ regime has not been reached yet for all $\kappa$ under consideration, especially for $\kappa \leq 0.15$ (Fig. 6.1). The data for $\kappa=0.3$ should be the most reliable one. Therefore the exponents of the viscosity $\eta_{n}$ and the first normal stress coefficient $\Psi_{n}^{(1)}$ are estimated from the data for $\kappa=0.3$ as

$$
b_{\eta} \approx 0.11 \quad \text { and } \quad b_{\Psi} \approx 0.77 .
$$

Within the accuracy of the data, the exponents are the same for both Erdôs-Rényi random graphs and three-dimensional percolation. This is similar to the Rouse model where the corresponding exponents of the viscosity and the first normal stress coefficient are nearly identical as a consequence of $d_{\mathrm{s}} \approx 4 / 3$ valid for Erdôs-Rényi random trees and bond percolation (cf. Eq. 5.22).

For the critical behavior of the overall viscosity (6.51) this implies a finite value at the gel point for both, Erdős-Rényi random graphs and three-dimensional bond percolation. This follows from (5.13) which requires the condition $b>\tau-2$ for a divergence. For $b_{\eta} \approx 0.11$ this is neither fulfilled for $\tau=5 / 2$ for Erdôs-Rényi random graphs nor for $\tau=2.18$ for bond percolation.

In contrast, by employing $(5.13)$ it is found that the first normal stress coefficient $\Psi^{(1)}$ diverges with an exponent that depends on the cluster statistics

$$
\ell=\left\{\begin{array}{ll}
0.54, & \text { Erdős-Rényi random graphs } \\
1.3, & \text { Bond percolation }(d=3)
\end{array} .\right.
$$


These exponent values are less than a third in magnitude of the corresponding exact analytical predictions $\ell=3$, respectively $\ell \approx 4.1$ of the Rouse model with the corresponding cluster statistics (cf. Sec. 6.1.1,6.1.2)

\subsubsection{Recoverable compliance}

The critical behavior of the recoverable compliance $J(4.43)$ is now deduced from the numerical results for $\eta$ and $\Psi^{(1)}$. Due to a finite viscosity the recoverable compliance has the same critical exponents $(6.56)$ as the first normal stress coefficient

$$
J \sim \varepsilon^{-\theta}
$$

with

$$
\theta=\left\{\begin{array}{ll}
0.54, & \text { Erdős-Rényi random graphs } \\
1.3, & \text { Bond percolation }(d=3)
\end{array} .\right.
$$

\subsubsection{Cluster diffusion}

Next, diffusion constants in the Zimm model are considered. In the Zimm model the diffusion constant (4.46) of a cluster is a more interesting quantity than in the Rouse model (cf. Eq. 6.19) since in the presence of hydrodynamic interactions $(H \neq \mathbb{1})$ it depends on the topology of the cluster .

\subsubsection{Kirkwood diffusion constant}

First, the averaged Kirkwood diffusion constant (4.47) at the critical point is considered

$$
D_{n}^{(\mathrm{K})}:=\left.\left\langle D^{(\mathrm{K})}(\cdot)\right\rangle_{n}\right|_{\varepsilon=0} \cdot
$$

Here, it is useful to compute the preaveraged mobility matrix $H$ (2.29) from the Oseen tensor with $h(x)=\sqrt{x / \pi}$. Then the average (6.59) can be expressed in terms of the $p-$ th moment $\rho_{n}^{(p)}$ of the resistance distribution (5.21) by setting $p=-1 / 2$

$$
D_{n}^{(\mathrm{K})}=\left.\frac{k_{\mathrm{B}} T}{\zeta}\left\langle\frac{1}{n}+\frac{\kappa}{n^{2}} \sum_{i, j \in(\cdot)} \mathscr{R}_{i, j}^{-\frac{1}{2}}\right\rangle_{n}\right|_{\varepsilon=0}=\frac{k_{\mathrm{B}} T}{\zeta}\left(\frac{1}{n}+\kappa \rho_{n}^{(-1 / 2)}\right) .
$$

By inserting the scaling relation (5.32) for $p=1 / 2$ and using the definition of the Gaussian spectral dimension $d_{\mathrm{f}}^{(\mathrm{G})}(6.8)$ the disorder averaged Kirkwood diffusion constant at the critical point is written as

$$
D_{n}^{(\mathrm{K})}=\frac{k_{\mathrm{B}} T}{\zeta}\left(\frac{1}{n}+\kappa \lambda n^{-1 / d_{\mathrm{f}}^{(\mathrm{G})}}\right)
$$

where $\lambda$ denotes an uninteresting numerical prefactor. 
Due to the two competing $n$ dependencies Eq. (6.61) predicts a crossover from Rouse behavior $D_{n}^{(\mathrm{K})} \sim n^{-1}$ for small $n$ to Zimm behavior

$$
D_{n}^{(\mathrm{K})} \sim n^{-1 / d_{\mathrm{f}}^{(\mathrm{G})}}
$$

for large $n$. The crossover cluster size $\widehat{n}(\kappa)$ depends on the strength of the hydrodynamic interactions $\kappa$. By matching the asymptotic formulas one has

$$
\widehat{n}(\kappa) \sim \kappa^{-d_{\mathrm{f}}^{(\mathrm{G})} /\left(d_{\mathrm{f}}^{(\mathrm{G})}-1\right)} .
$$

The crossover cluster size $\widehat{n} \approx \pi / \kappa^{2}$ which has been found for the viscosity of ring polymers (see. Sec. 6.2.1.2) is compatible with (6.63) since ring polymers have Gaussian fractal dimension $d_{\mathrm{f}}=2$.

The result (6.62) for the diffusion constant is reasonable. In can be written in terms of the averaged radius of gyration of phantom percolation clusters (6.9) according to

$$
D_{n}^{(\mathrm{K})} \sim 1 / \sqrt{R_{\mathrm{gyr}, n}^{2}} .
$$

In other words, the averaged diffusion constant is inversely proportional to the averaged radius of gyration which is nothing but the Stokes-Einstein relation (1.14). This scaling is well-known to be valid in the Zimm model for linear polymers. Linear polymers have Gaussian fractal dimension 2 and a diffusion constant $D_{\operatorname{lin}, n}^{(\mathrm{K})} \sim n^{-1 / 2}$ [DoEd88].

\subsubsection{Diffusion constants}

More detailed information on $D_{n}$ is obtained from numerical studies. The averaged diffusion constant

$$
D_{n}:=\left.\langle D\rangle_{n}\right|_{\varepsilon=0} \sim n^{-b_{D}}
$$

of clusters of size $n$ at the gel point is considered. The exponent $b_{D}$ which governs the scaling for large cluster sizes $n$, is bounded from below by the corresponding exponent of the Kirkwood diffusion constant

$$
b_{D} \geq 1 / d_{\mathrm{f}}^{(\mathrm{G})}
$$

which is a consequence of the the Jensen-Peierls inequality $D_{n} \leq D_{n}^{(\mathrm{K})}$ (cf. Eq. (4.49)).

Erdôs-Rényi random trees For each $n=1 \ldots 750$ the average $D_{n}$ is computed over 100 realizations of trees of size $n$. In Fig. 6.4(a) $D_{n}$ is plotted as a function of $n$ on a doublelogarithmic scale for different values of the hydrodynamic interaction parameter $\kappa$. The exponent $b_{D}$ is extracted by fitting the curves to a power law in the interval $n \in[700,750]$. Fig. 6.4(b) displays the exponent $b_{D}$ for different $\kappa$. The horizontal line marks the lower bound $b_{D}>1 / d_{\mathrm{f}}^{(\mathrm{G})}=1 / 4$ from the Kirkwood diffusion constant. A sharp crossover is observed from the Rouse value $b_{D}=1$ (cf. 6.19) for $\kappa=0$ to smaller values of $b_{D}$ for nonzero $\kappa$. The latter are close to and may be identical to the lower bound $1 / d_{\mathrm{f}}^{(\mathrm{G})}=1 / 4$. 

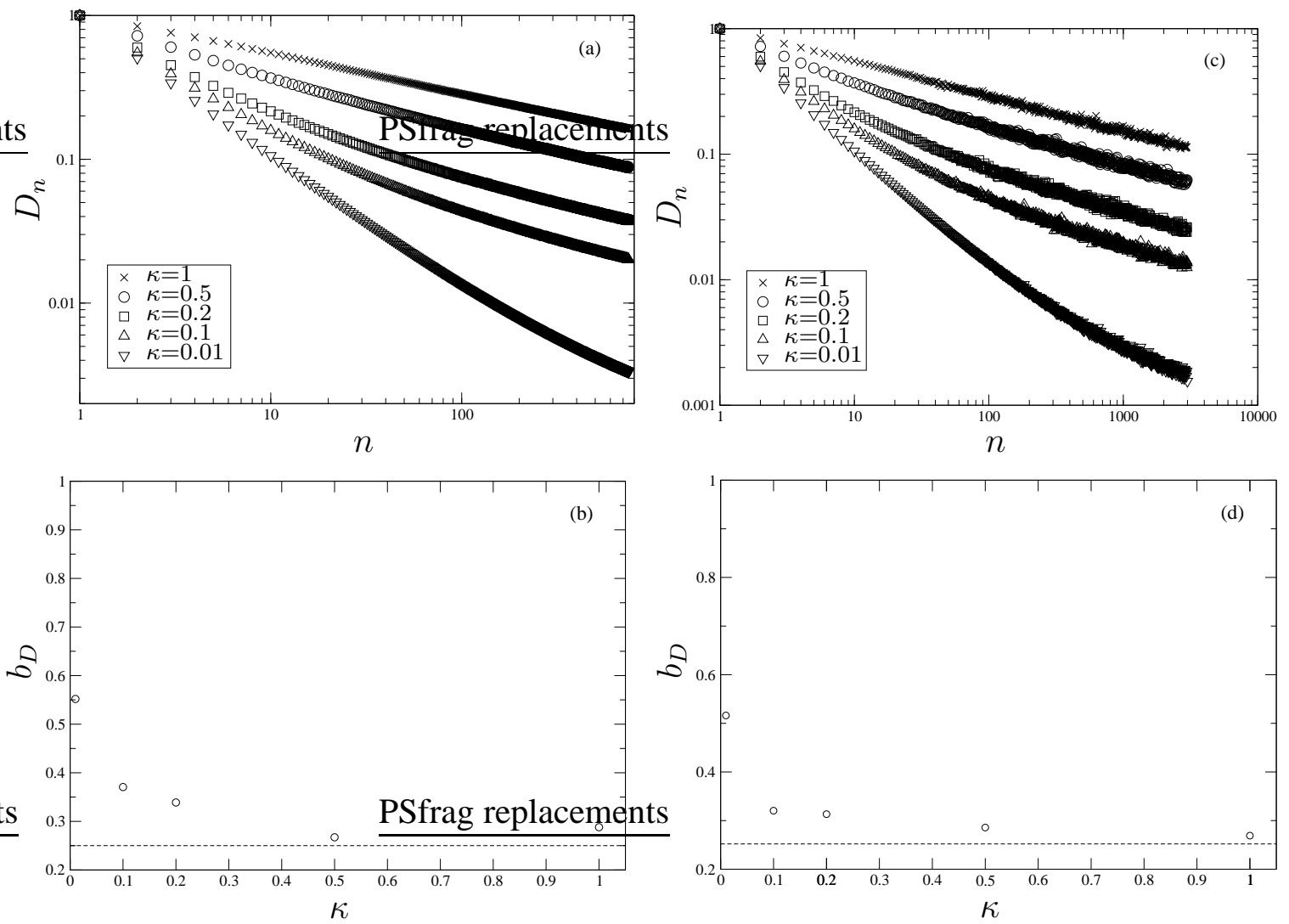

Figure 6.4: Numerical data to determine the scaling (6.65) of the diffusion constant for random clusters in the case of Erdôs-Rényi random graphs (left column) and three-dimensional bond percolation (right column). In each case the diffusion constant $D_{n}$ is plotted for different strengths of the hydrodynamic interaction parameter $\kappa$ as a function of the cluster size $n$ on a double logarithmic scale. Power-law fi ts to the data yield the exponent $b_{D}$ a function of $\kappa$ (bottom). 
Bond percolation For bond percolation cluster sizes are restricted to values $n<3000$ due to the memory limitations when computing the inverse of $H$. For small cluster sizes up to 100 diffusion constants are computed for given $n$, for large cluster sizes this number is considerably smaller. The results are shown in Figs. 6.4(c) and 6.4(d). In Fig. 6.4(c) $D_{n}$ is plotted as a function of $n$ on a double-logarithmic scale for different values of $\kappa$. The exponent $b_{D}$, extracted by fitting the curves in Fig. 6.4(c) to a power law in the interval $n \in[500,3000]$, is shown in Fig. 6.4(d). The horizontal line marks the lower bound $1 / d_{\mathrm{f}}^{(\mathrm{G})} \approx 0.25$ for $b_{D}$, based on the value $d_{s} \approx 1.33$ [NaYa94, BuHa96]. Again one may conjecture that $b_{D}=1 / d_{\mathrm{f}}^{(\mathrm{G})}$. The numerical values of $b_{D}$ for bond percolation are nearly identical to those obtained for Erdős-Rényi random graphs.

In summary, the numerical data for both ensembles shows a crossover from initial Rouse behavior to Zimm behavior which is more pronounced for small $\kappa$. This is very similar to the observed crossover for the viscosity and the first normal stress coefficient. However, for the diffusion constants an upper bound, the Kirkwood diffusion constant can be computed analytically. The exponent $d_{\mathrm{f}}^{(\mathrm{G})}$ characterizing the bound, agrees qualitatively with the numerical values for $b_{D}$. Therefore, it is suggested that

$$
b_{D}=1 / d_{\mathrm{f}}^{(\mathrm{G})} .
$$

The suggestion, that the diffusion constant and the Kirkwood diffusion constant have the same scaling with cluster size $n$ is also substantiated by results for the Zimm model of linear polymers where the scaling with $n$ for both diffusion constants is governed by the same exponent $D_{\mathrm{lin}, n}^{(\mathrm{K})} \sim D_{\mathrm{lin}, n} \sim n^{-1 / 2}$ (see [Oet87b]).

\subsubsection{Incoherent scattering function}

Within the Zimm model it is only possible to compute the incoherent scattering function in the asymptotic regime on the longest time scales $t \rightarrow \infty$. The disorder average of the bound (4.54) is given by

$$
\left.S(\boldsymbol{q}, t) \sim \sum_{n=1}^{\infty} n \tau_{n}\left\langle\mathrm{e}^{-D\left(\mathscr{N}_{k}\right) q^{2} t}\right\rangle_{n}\right|_{\varepsilon=0} .
$$

By applying the Jensen inequality, the average $\langle\bullet\rangle_{n}$ is shifted into the argument of the exponential

$$
S(\boldsymbol{q}, t) \sim \sum_{n=1}^{\infty} n \tau_{n} \mathrm{e}^{-D_{n} q^{2} t} \geq \sum_{n=1}^{\infty} n \tau_{n} \mathrm{e}^{-D_{n}^{(K)} q^{2} t}
$$

where the second inequality employs $D_{n} \leq D_{n}^{(\mathrm{K})}$. The expression (6.69) can be evaluated in the limit $t \rightarrow \infty$ (see App. E.1). Up to a multiplicative constant, this yields the scaling form

$$
S(\boldsymbol{q}, t) \sim\left(q^{2} t\right)^{-\delta} \mathcal{F}_{S}\left(t / t_{q}\right)
$$




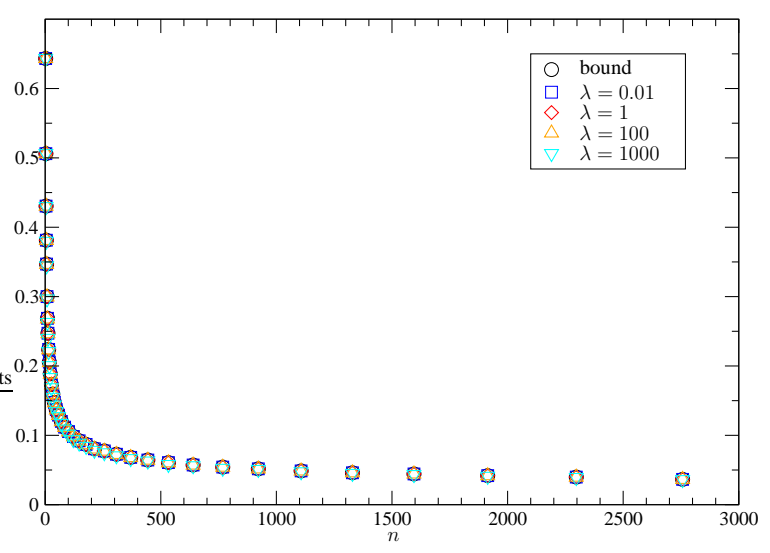

Figure 6.5: Estimating the tightness of the Jensen inequality which is employed for the incoherent scattering function. For Erdős-Rényi random graphs the averaged diffusion constant $\langle D\rangle_{n}$ is compared with $-1 / \lambda \log \langle\exp \{-\lambda D\}\rangle_{n}$ for $\kappa=0.3$, where $\lambda$ is varied over different orders of magnitude.

with a diverging, diffusive time scale

$$
t_{q} \sim q^{-2} \varepsilon^{-\tilde{z}}, \quad \text { with } \quad \tilde{z}=\nu^{(\mathrm{G})}
$$

and a scaling function $\mathcal{F}_{S}(x)$ which is of order unity for $x \rightarrow 0$ and decays like a stretched exponential $\mathcal{F}_{S}(x) \sim x^{\alpha_{S}(\delta-1 / 2)} \exp \left\{-c x^{\alpha_{S}}\right\}$ for $x \rightarrow \infty$. Neglecting algebraic prefactors, the incoherent scattering function has a stretched exponential decay

$$
S(\boldsymbol{q}, t) \sim \exp \left\{-\left(t / t_{q}\right)^{\alpha_{S}}\right\}, \quad \text { with } \quad \alpha_{S}=d_{\mathrm{f}}^{(\mathrm{G})} /\left(d_{\mathrm{f}}^{(\mathrm{G})}+1\right)
$$

in the sol phase for $c<c_{\text {crit }}$ and and a power law decay

$$
S(\boldsymbol{q}, t) \sim \frac{1}{\left(q^{2} t\right)^{\delta}}, \quad \text { with } \quad \delta=(\tau-2) d_{\mathrm{f}}^{(\mathrm{G})}
$$

at the critical point $c=c_{\text {crit }}$ [KuLo03]. The equations (6.70-6.73) for the Zimm model are to be compared with the results $(6.29-6.32)$ for the Rouse model. Here, in contrast to the Rouse model, it can only be suggested that the expressions (6.69) and (6.68) have the same long time behavior. In order to support the suggestion, the tightness of the Jensen inequality has been checked numerically (see Fig. 6.5). Within numerical accuracy the Jensen inequality becomes an equality and hence does not affect the long-time behavior.

The knowledge of the diffusive time scale (6.71) provides a way to estimate the longest relaxation time $t^{*}$ in the Zimm model with the same line of argument presented below Eq. (6.35). Since static properties of the Zimm model are identical to the Rouse model, the critical length scale should also be given by $\xi^{*}$. Then, dimensional analysis dictates $t_{q} \sim$ $t^{*} /(q \xi)^{2} \sim \varepsilon^{2 \nu^{(\mathrm{G})}-z}$ in terms of the Zimm relaxation time $t^{*}$. Comparing this expression with (6.71) leads to

$$
t^{*} \sim \varepsilon^{-z}, \quad \text { with } \quad z=3 \nu^{(\mathrm{G})} .
$$


This scaling is in agreement with the simple estimate that the longest relaxation time is determined by the diffusive time scale of the largest clusters $t^{*} \sim\left(\xi^{*}\right)^{2} / D_{n^{*}} \sim\left(\xi^{*}\right)^{2} R_{n^{*}} \sim$ $\left(\xi^{*}\right)^{3}$.

\subsubsection{Effective diffusion constant}

The effective diffusion constant (3.29) is calculated from the bound (6.69)

$$
D_{\text {eff }}^{-1}=\left\langle D_{\text {eff }}^{-1}(\cdot)\right\rangle \geq \sum_{n=1}^{\infty} n \tau_{n}\left(D_{n}^{(\mathrm{K})}\right)^{-1} .
$$

From $D_{n}^{(\mathrm{K})} \sim n^{1 / d_{\mathrm{f}}^{(\mathrm{G})}}$ and Eq. (5.12) one concludes, that the effective diffusion constant $D_{\text {eff }}$ from (3.29) vanishes

$$
D_{\text {eff }} \lesssim \varepsilon^{u}, \quad \text { with } \quad u=\left(2-\tau+1 / d_{\mathrm{f}}^{(\mathrm{G})}\right) / \sigma,
$$

provided that $u>0$. Three-dimensional bond percolation leads to the value $u \approx 0.16$. If instead $1 / d_{\mathrm{f}}^{(\mathrm{G})}<\tau-2$, then $D_{\text {eff }}$ remains nonzero at the transition. Such an unphysical situation will occur for Erdős-Rényi random graphs. Note that the average time as exemplified by $D_{\text {eff }}$ is not proportional to the time scale $t_{q}$ of the stretched exponential, as is sometimes assumed incorrectly. The effective diffusion constant can be interpreted as the diffusion constant of the cluster with weight-averaged cluster size $D_{n_{\mathrm{w}}} \sim n_{\mathrm{w}}^{-1 / d_{\mathrm{f}}^{(\mathrm{G})}} \sim \varepsilon^{\gamma / d_{\mathrm{f}}^{(\mathrm{G})}} \sim \varepsilon^{u}$. 


\section{Chapter 7}

\section{Discussion}

\subsection{Summary of results}

In this work results have been obtained for the critical dynamics of gelation by starting from the well known semi-microscopic dynamical description of polymers within the Rouseand the Zimm model which is generalized to random clusters. The cluster statistics is chosen according to either Erdős-Rényi random graphs pertaining to a mean field distribution which neglects correlations between crosslinks or $3 d$ bond percolation. Within these models observables are examined which show critical behavior at the gelation transition. Critical rheology is revealed by the shear viscosity $\eta$, the first normal stress coefficient $\Psi^{(1)}$ and the recoverable compliance $J$. The second normal stress coefficient $\Psi^{(2)}$ vanishes in both models. Critical, microscopic density fluctuations are revealed by diffusion constants $D_{n}$ of single clusters of size $n$, the incoherent scattering function $S(\boldsymbol{q}, t)$, the inverse, effective diffusion constant $D_{\text {eff }}^{-1}$ and the nonlinear dynamic susceptibility $\chi(\boldsymbol{q}, t)$. The critical behavior of the observables is summarized in Tab. 7.1. Additionally, results from [BrGo97, BrAs01, Mue03, KiMu04] are given since they complete the picture of the gelation transition in these models. Instead of expressing critical exponents strictly in terms of the fundamental exponents $\sigma, \tau, d_{\mathrm{s}}$ it will be useful for later discussions to pick appropriate ones from $\sigma, \tau, \gamma, \beta, d_{\mathrm{s}}, d_{\mathrm{f}}^{(\mathrm{G})}, \nu^{(\mathrm{G})}$ by employing the scaling relations $2 / d_{\mathrm{f}}^{(\mathrm{G})}=2 / d_{\mathrm{s}}-1$, $\nu^{(\mathrm{G})}=\left(\sigma d_{\mathrm{f}}^{(\mathrm{G})}\right)^{-1}$ and the scaling relations of the connectivity properties of percolation from Tab. 1.1.

For the Rouse model which neglects excluded volume and hydrodynamic interactions, analytical results have been obtained by means of exact mappings of the Rouse dynamics onto resistor networks and onto random walks on fractals. The so obtained results can be discussed simultaneously for both crosslink ensembles, Erdős-Rényi random graphs and $3 d$ bond percolation since the first is recoverd by the latter in its mean field approximation for $d \geq 6$.

By employing the resistance mapping the zero-shear material functions have been computed. These quantities only require the knowledge of the shear response on the longest time scales. The Mark-Houwink relation $\eta_{n} \sim n^{b_{\eta}}$ for the averaged viscosity of clusters of 


\begin{tabular}{|c|c|c|c|c|c|c|}
\hline Quantity & Scaling & Exp. & Rouse & $d=3$ & Zimm & $d=3$ \\
\hline Viscosity & $\begin{array}{l}\eta_{n} \sim n^{b_{\eta}} \\
\eta \sim \varepsilon^{-k}\end{array}$ & $\begin{array}{l}b_{\eta} \\
k\end{array}$ & $\begin{array}{l}2 / d_{\mathrm{f}}^{(\mathrm{G})} \\
2 \nu^{(\mathrm{G})}-\beta\end{array}$ & $\begin{array}{l}0.50 \\
0.71\end{array}$ & - & $\begin{array}{l}0.11 \\
-\end{array}$ \\
\hline $\begin{array}{l}\text { Normal stress } \\
\text { coeffi cient }\end{array}$ & $\begin{array}{l}\Psi_{n}^{(1)} \sim n^{b_{\Psi}} \\
\Psi^{(1)} \sim \varepsilon^{-\ell}\end{array}$ & $\begin{array}{l}b_{\Psi} \\
\ell\end{array}$ & $\begin{array}{l}4 / d_{\mathrm{f}}^{(\mathrm{G})}+1 \\
4 \nu^{(\mathrm{G})}+\gamma\end{array}$ & $\begin{array}{l}2.01 \\
4.04\end{array}$ & - & $\begin{array}{l}0.54 \\
1.30\end{array}$ \\
\hline $\begin{array}{l}\text { Recoverable } \\
\text { complicance }\end{array}$ & $\begin{array}{l}J_{n} \sim n^{b_{J}} \\
J \sim \varepsilon^{-\theta}\end{array}$ & $\begin{array}{l}b_{J} \\
\theta\end{array}$ & $\begin{array}{l}2 \tau-3 \\
(\tau-1) / \sigma\end{array}$ & $\begin{array}{l}1.36 \\
2.62 \\
\end{array}$ & - & $\begin{array}{l}0.54 \\
1.30 \\
\end{array}$ \\
\hline $\begin{array}{l}\text { Shear } \\
\text { relaxation } \\
\text { function }\end{array}$ & $\begin{aligned} G(t) & \sim t^{-\Delta} \mathcal{F}_{G}\left(t / t^{*}\right) \\
t^{*} \sim \varepsilon^{-z} & \\
G(t) & \sim \mathrm{e}^{-\left(t / t^{*}\right)^{-\alpha}} \quad \quad \quad c<c_{\text {crit }} \\
G(t) & \sim t^{-\Delta}, \quad c=c_{\text {crit }}\end{aligned}$ & $\begin{array}{l}z \\
\alpha_{G} \\
\Delta\end{array}$ & $\begin{array}{l}k+\theta \\
1 / 3 \\
\theta /(k+\theta)\end{array}$ & $\begin{array}{l}3.33 \\
0.33 \\
0.79\end{array}$ & $\begin{array}{l}3 \nu^{(\mathrm{G})} \\
-\end{array}$ & $\begin{array}{l}1.68 \\
-\end{array}$ \\
\hline $\begin{array}{l}\text { Incoherent } \\
\text { scattering } \\
\text { function }\end{array}$ & $\begin{array}{l}S(q, t) \sim\left(q^{2} t\right)^{-\delta} \mathcal{F}_{S}\left(t / t_{q}\right) \\
t_{q} \sim q^{-2} \varepsilon^{-\tilde{z}} \\
S(q, t) \sim \mathrm{e}^{-\left(t / t_{q}\right)^{-\alpha_{S}}}, \quad c<c_{\mathrm{crit}} \\
S(q, t) \sim t^{-\delta}, \quad c=c_{\mathrm{crit}}\end{array}$ & $\begin{array}{l}\tilde{z} \\
\alpha_{S} \\
\delta\end{array}$ & $\begin{array}{l}1 / \sigma \\
1 / 2 \\
\tau-2\end{array}$ & $\begin{array}{l}2.22 \\
0.5 \\
0.18\end{array}$ & $\begin{array}{l}\nu^{(\mathrm{G})} \\
d_{\mathrm{f}}^{(\mathrm{G})} /\left(d_{\mathrm{f}}^{(\mathrm{G})}+1\right) \\
\beta / \nu^{(\mathrm{G})}\end{array}$ & $\begin{array}{l}0.56 \\
0.80 \\
0.71\end{array}$ \\
\hline $\begin{array}{l}\text { Diffusion } \\
\text { constant }\end{array}$ & $D_{n} \sim n^{-b_{D}}$ & $b_{D}$ & 1 & 1.0 & $1 / d_{\mathrm{f}}^{(\mathrm{G})}$ & 0.25 \\
\hline $\begin{array}{l}\text { Eff. diffusion } \\
\text { constant }\end{array}$ & $D_{\mathrm{eff}}^{-1} \sim \varepsilon^{-u}$ & $u$ & $\gamma$ & 1.80 & $\nu^{(\mathrm{G})}-\beta$ & 0.15 \\
\hline
\end{tabular}

Table 7.1: Overview of critical behaviour in the Rouse and in the Zimm model. Numerical values in $d=3$ have been obtained from the percolation exponents given in Tab. 1.1 and the numerical values $d_{\mathrm{f}}^{(\mathrm{G})}=2 d_{\mathrm{s}}\left(2-d_{\mathrm{s}}\right)^{-1} \approx 3.97$ and $\nu^{(\mathrm{G})}=\left(\sigma d_{\mathrm{f}}^{(\mathrm{G})}\right)^{-1} \approx 0.56$, which have been computed from $d_{\mathrm{s}} \approx 1.33$ (see Tab. 5.1).

size $n$ at the critical point has been computed analytically. The Mark-Houwink exponent $b_{\eta}$ is given in terms of the spectral dimension $d_{\mathrm{s}}$ of percolation clusters, which chracterizes the connectivity of the clusters for large topological distances without referring to the spatial configuration. The overall shear viscosity $\eta$ of the gelling system is then determined by weighting these contributions from clusters of size $n$ according to the cluster size distribution of the respective crosslink ensembles, which are governed by the exponents $\tau, \sigma$. Thereby, the critical exponent $k$ of the viscosity is expressed in terms of the basis set of three independent exponents $\tau, \sigma$, and $d_{\mathrm{s}}$. The additive decomposition of an observable into contributions from different clusters is a generic feature of the Rouse model and valid only in the absence of cluster-cluster interactions. The resistance analogy is also applied to suggest a Mark-Houwink like scaling relation $\Psi_{n}^{(1)} \sim n^{b_{\Psi}}$ for the averaged contribution of clusters of size $n$ to the first normal stress coefficient at the critical point. The critical exponent $\ell$ governing the divergence of the overall first normal stress coefficient is expressed in terms of $\tau, \sigma$, and $d_{\mathrm{s}}$. The elastic response of the system on the longest time scale as revealed by the recoverable compliance $J$ can be related to the viscosity and the first normal stress coefficient. In contrast to the latter quantities the critical exponent $\theta$ of $J$ does not involve the spectral dimension $d_{\mathrm{s}}$, it is completely determined by the exponents $\tau, \sigma$ of the underlying clusters size distribution. 
As already mentioned, concerning material functions it suffices to compute the shear response on the longest time scales as revealed by the lowest order moments of the distribution of inverse relaxation times, the shear relaxation function $G(t)$. If instead, the complete spectrum of relaxation times is addressed by employing results from [Mue03, BrAs01, KiMu04], one has also access to functional forms of relaxation patterns such as the stretched exponential decay of the shear relaxation function in the sol phase, the algebraic decay at the critical point or the general scaling form $G(t) \sim t^{-\Delta} \mathcal{F}_{G}\left(t / t^{*}\right)$ with the divergent time scale $t^{*} \sim \varepsilon^{-z}$. The exponents $z, \Delta$ are given in terms of $\sigma, \tau, d_{\mathrm{s}}$. In particular, the knowledge of the scaling exponents $z, \Delta$ has been exploited twofold in this work. First, the fundamental scaling relations of critical rheology (cf. Sec. 1.3.1) can be verified. The viscosity exponent $k$ satisfies $k=z(1-\Delta)$ which in accordance with the scaling hypothesis. The critical exponent $\theta$ of the recoverable compliance satisfies $\theta=z \Delta$ which is also in accordance with the scaling hypothesis, since the recoverable compliance plays the role of an inverse shear modulus. Second, and even more important, the knowledge of the characteristic time scale $t^{*} \sim \varepsilon^{-z}$ in the Rouse model allows to extend the scaling picture of critical relaxations to time and space dependent processes. By computing bounds to the incoherent scattering function $S(\boldsymbol{q}, t)$ and the nonlinear dynamic susceptibility $\chi(\boldsymbol{q}, t)$ the characteristic length scale $\xi^{*} \sim \varepsilon^{-\nu^{(\mathrm{G})}}$ of the Rouse model, the Gaussian correlation length, has been identified. Its exponent $\nu^{(\mathrm{G})}$ is related to the Gaussian fractal dimension $d_{\mathrm{f}}^{(\mathrm{G})}$ in the same way as the correlation length exponent $\nu$ of rigid percolation is related to the Hausdorff fractal dimension $\nu=1 /\left(\sigma d_{\mathrm{f}}^{(\mathrm{H})}\right)$. Both, the bound to the incoherent scattering function $S(\boldsymbol{q}, t) \gtrsim s\left(q \xi^{*}, t / t^{*}\right)$ and the bound to the nonlinear dynamic susceptibility, $\chi(\boldsymbol{q}, t) \gtrsim \varepsilon^{-\gamma} \mathcal{F}_{\chi}\left(q \xi^{*}, t / t^{*}\right)$ can be written in terms of the scaling variables $t / t^{*}$ and $q \xi^{*}$ for all times $t$ which are large when compared to the microscopic time scale. The relevance of these two scales is also reflected by the asymptotic, long-time decay of the incoherent scattering function for $t \rightarrow \infty$. It is governed by a stretched exponential $S(\boldsymbol{q}, t) \sim \exp \left\{-\left(t / t_{q}\right)^{\alpha_{S}}\right\}$. The characteristic, diffusive time scale $t_{q}$ is related to the fundamental scales according to $t_{q}=t^{*} /\left(q \xi^{*}\right)^{2}$.

In the strict limit $t \rightarrow \infty$ the scattering function recovers the order parameter $S_{\infty}(\boldsymbol{q})$ of gelation in the Rouse model. It is zero in the sol phase for $c<c_{\text {crit }}$ and acquires a time persistent part in the gel phase which is reminescent of the Debye-Waller factor. In the vicinity of the transition, its $q$-dependence is determined by a distribution of localization lengths of monomers in the macroscopic cluster. Again, the Gaussian correlation length $\xi^{*}$ sets the characteristic scale of this distribution. However, it has been pointed out that the physical meaning of the gel phase in the Rouse model is unclear.

For the Zimm model, which neglects excluded volume interactions and accounts for hydrodynamic interactions on a preaveraged level, results are mainly obtained numerically. By generating clusters at the critical point according to Erdős-Rényi random graphs and bond percolation the disorder averages of the viscosity and the first normal stress coefficient are computed numerically. The numerical results for random clusters are considerably affected by finite size effects. The comparison with numerical data for ring clusters allows to corroborate the identified critical exponents. It has been found that, within nu- 
merical accuracy, the Mark-Houwink relation $\eta_{n} \sim n^{b_{\eta}}$ is governed by the same exponent $b_{\eta}$ in both ensembles, Erdős-Rényi random graphs and bond percolation. As a consequence of the small value of $b_{\eta}$ the viscosity $\eta$ remains finite at the critical point in either case. In contrast, the numerically computed Mark-Houwink like scaling exponent $b_{\Psi}$ for the first normal stress coefficient leads to a divergent normal stress coefficient $\Psi^{(1)}$ at the critical point, where the exponent $\ell$ depends on the clusters statistics. The recoverable compliance $J$ diverges with the same exponent due to a finite value of the viscosity.

Density fluctuations in the Zimm model can be studied from the incoherent scattering function on the longest time scales. The dominating contribution stems from the diffusion of clusters and can be computed from the scaling of the averaged diffusion constant $D_{n}$ of clusters of size $n$. The scaling $D_{n} \sim n^{-b_{D}}$ has been obtained numerically for both crosslink statistics. Similar to the viscosity, finite size effects prohibit an accurate determination of $b_{D}$. However, the results can be compared to an upper bound, the averaged Kirkwood diffusion constant $D_{n}^{\mathrm{K}}$ which has been computed analytically. The qualitative agreement of $D_{n}$ with $D_{n}^{\mathrm{K}}$ leads to the conclusion that both diffusion constants are governed by the same exponent $b_{D}=1 / d_{\mathrm{f}}^{(\mathrm{G})}$.

By employing the scaling of the diffusion constant $D_{n}$ to the incoherent scattering function, a stretched exponential decay $S(\boldsymbol{q}, t) \sim \exp -\left(t / t_{q}\right)^{\alpha_{S}}$ in the sol phase is obtained with a characteristic, diffusive time scale $t_{q}$ which diverges at the critical point. The exponent $\alpha_{S}$ is the same for both crosslink ensembles. Precisely at the critical point the decay of $S(\boldsymbol{q}, t)$ is algebraic in time. The effective diffusion constant $D_{\text {eff }}^{-1}$ which is obtained by integrating the incoherent scattering function is found to diverge only for $3 d$ bond percolation.

Having determined the exponent $\tilde{z}$ of the diverging time scale $t_{q} \sim \varepsilon^{-\tilde{z}}$ it allows to identify the exponent $z$ of the characteristic time scale $t^{*}$ in the Zimm model by dimensional analysis $t_{q} \sim t^{*} /\left(q \xi^{*}\right)$. The length scale in the Zimm model is also given by the Gaussian correlation length $\xi^{*}$ since static properties, and hence length scales, are the same as in the Rouse model.

In summary, the Rouse model of gelation enables one to derive a rather complete scaling picture of the transition in the sol phase. Scaling relations can be computed exactly in terms of the characteristic time scale $t^{*}$ and a characteristic length scale $\xi^{*}$. These scales are governed by critical exponents which are not solely determined by the two exponents of percolation. For the Zimm model of gelation far less analytical predictions are available. Some scaling relations can be given in terms of the basic exponents, for others only numerical values are available.

Now the results shall be discussed against the background of existing experimental and theoretical results. First the experimental results are reviewed. Afterwards, the derived scaling relations for the Rouse and the Zimm model are compared with existing scaling relations. 


\begin{tabular}{|c|c|c|c|c|c|c|c|}
\hline & $k$ & $\ell$ & $\theta$ & $z$ & $\Delta$ & $\alpha_{G}$ & $\mu$ \\
\hline Rouse $\left(\nu^{(\mathrm{G})}\right)$ & 0.71 & 4.05 & 2.62 & 3.3 & 0.79 & $1 / 2$ & - \\
\hline $\operatorname{Zimm}\left(\nu^{(\mathrm{G})}\right)$ & 0 & 1.3 & 1.3 & - & - & - & - \\
\hline Rouse $(\nu)$ & 1.35 & - & 2.64 & 3.99 & 0.66 & - & 2.64 \\
\hline $\operatorname{Zimm}(\nu)$ & 0 & - & 2.64 & 2.64 & 1 & - & 2.64 \\
\hline [AxKo90] & 0.82 & & & 2.67 & 0.71 & & 1.93 \\
\hline [DeB093] & 0.7 & & & 2.9 & 0.72 & & 2.0 \\
\hline [TaY094] & 1.3 & & $2.7-2.8$ & & $0.67-0.68$ & & $2.0-2.1$ \\
\hline [LuMo95] & 1.36 & & 2.71 & & 0.66 & & \\
\hline [LuMo99] & 6.1 & & 3.2 & & 0.33 & & \\
\hline [DuDe87] & & & & & 0.70 & & \\
\hline [AdDe79] & 0.78 & & & & & & \\
\hline [AdDe85] & 0.81 & & & & & & 3.2 \\
\hline [AdLa97] & 1.1 & & & & 0.69 & & 1.9 \\
\hline [ToFa01] & 0.76 & & & & 0.77 & & 2.54 \\
\hline [TaUr90] & 0.2 & & & & & & \\
\hline [MaAd88] & 1.4 & & & & 0.70 & & \\
\hline [CoGi93] & $>1.4$ & & & & & & 3.0 \\
\hline [ZhTh96] & 1.27 & & & & & & \\
\hline [TiTo04] & & & & & $0.69-0.77$ & & $2.4-2.7$ \\
\hline
\end{tabular}

Table 7.2: Experimental values for the viscoelastic exponents. Additionally numerical values of the theoretical predictions of the Rouse and Zimm model from this work, referred to as Rouse $\left(\nu^{(\mathrm{G})}\right)$ and Zimm $\left(\nu^{(\mathrm{G})}\right)$, and theoretical predicitons from scaling arguments, referred to as Rouse $(\nu)$ and Zimm $(\nu)$ are given for comparison. The latter theoretical predictions are discussed in Sec. 7.3 where the naming conventions will become clear. Numerical RouseZimm values have been obtained from the percolation exponents given in Tab. 1.1 and the numerical value $\nu^{(\mathrm{G})}=\left(1 / d_{\mathrm{s}}-1 / 2\right) / \sigma \approx 0.56$ which has been computed from $d_{\mathrm{s}} \approx 1.33$ (see Tab. 5.1).

\subsection{Comparison to experiments and simulations}

Besides those experiments mentioned in the introduction which basically confirm that connectivity properties of gelling systems are well described by percolation (see also Tab. 3 in [AdLa96]), critical rheology and in particluar the shear viscosity are the most frequently studied quantities in experiments. Few experimental results are available for single cluster properties such as the Mark-Houwink relation for randomly branched polymers. Furthermore, microscopic density fluctuations have been studied by light scattering experiments. The experimental point of view is completed by computer simulations. All experimental results are listed in Tabs. 7.2 and 7.3. The first experiments which report on the scaling of complex shear relaxation function (see Sec. 1.3.1) were [ChWi85] who find $\Delta=0.5$. The validity of the fundamental scaling assumption $G(t) \sim t^{-\Delta} \mathcal{F}_{G}\left(t \varepsilon^{z}\right)$ within numerical accuracy has been confirmed for different systems. Devreux et al have investigated silica gels in [DeBo93], they confirm the validity of the scaling relations $z=k+\mu$ and 
$\mu=\Delta z$, where $\mu$ is the exponent of the shear modulus. Similarly, Axelos et al [AxKo90] have verified these scaling relations for a completely different system of crosslinked pectin biopolymers. The numerical values of their exponents are similar to those in [DeBo93]. Rather different exponents have been measured by Lusignan et al from polycondensation reactions of polyesters of different lengths [LuMo95, LuMo99]. Short polyesters where investigated in [LuMo95] and an exponent $\tau \approx 2.17$ was found which is well described by $3 d$ percolation. In contrast, for the second system of long chains [LuMo99] a mean field like exponent $\tau \approx 2.47$ was found in agreement with the vulcanization picture. The large value of $k$ in the latter experiment is argued to be influenced by entanglement effects. However, both experiments report on the validity of the scaling relations. Noteworthy, in both experiments the scaling law $\Delta=\theta /(\theta+k)$ was confirmed by measuring the recoverable compliance and its exponent $\theta$ rather than the shear modulus exponent $\mu$. Both quantities are argued to be characterized by the same critical exponent. However, the equivalence of the shear modulus and the inverse recoverable compliance is not obvious as indicated by the experiments reported in [TaYo94] for an end-linking reaction of polyoxypropylene. The exponent of the recoverable compliance is significantly higher than that of the shear modulus.

Moreover, in many experiments some but not all viscoelastic exponents have been measured. Adam et al studied a co-polymerization reaction of Styrene divinylbenzene [AdDe79] and reported $k=0.78$. A similar value $k=0.8$ has been found by some of the latter authors in a polycondensation reaction of polyurethane gels [AdDe85]. In [AdLa97] vinyl terminated PDMS networks are investigated and three viscoelastic exponents were measured independently. Rather different exponents for the same system were found in [ToFa01] and attributed to the variation of the functionality of the crosslinkers and the average degree of polymerization of the prepolymers. The influence of the chemical structure of crosslinkers and prepolymers was investigated by one of the latter authors in [TiTo04]. From the variation of the exponents $0.69 \leq \Delta \leq 0.77$ and $2.4 \leq \mu \leq 2.7$ the authors suggest a possible non-universal behavior of the critical exponents.The authors in [ZhTh96] studied an alginate system where gelation was induced by cupric ions and found $k=1.27$. Similar high values are obtained in expoxy resins [MaAd88]. The highest values for $k$, besides that in [LuMo99], have been found by Colby et al [CoGi93] where $k>1.4$.

The Mark-Houwink exponent $b_{\eta}$ has also been investigated experimentally for branched macromolecules. In Ref. [MaOh86] measurements on randomly branched polystyrenes have been performed, resulting in $b_{\eta} \in[0.2,0.25]$. Measurements on branched polyethyleneimine [PaCh96] yield the slightly higher value $b_{\eta} \approx 0.31$. Unfortunately, no results have been reported on the critical behaviour of the first normal stress coefficient.

The experimental values for the long time decay of the scattering function are summarized in Tab. 7.3. In fact, all three experiments measure the coherent scattering function (see Sec. 3.2.2). However, it is argued in [MaWi91] that only self-correlations are expected to contribute to the signal and the experimental results are therefore discussed in terms of the incoherent scattering function. Even though the validity of this approximation may be questioned it is expected that both, the coherent and the incoherent scattering are governed 


\begin{tabular}{l||c|c|c|c|} 
& $\alpha_{S}$ & $\delta$ & $\tilde{z}$ & $u$ \\
\hline \hline $\operatorname{Rouse}\left(\nu^{(\mathrm{G})}\right)$ & $1 / 2$ & 0.18 & 2.22 & 1.82 \\
$\operatorname{Zimm}\left(\nu^{(\mathrm{G})}\right)$ & 0.80 & 0.71 & 0.56 & 0.16 \\
[MaWi91] & 0.66 & 0.27 & 2.5 & 1.9 \\
[AdDe88] & $0.3-0.8$ & $0.2-0.3$ & - & $0.5-1.0$ \\
[BaBu92] & 0.64 & 0.34 & - & 1.9
\end{tabular}

Table 7.3: Critical exponents for the incoherent scattering function.

by the same stretched exponential decay for long times. For the Rouse model this claim can be substantiated by non-rigorous arguments [Kue01].

Also, a variety of computer simulation have been performed in order to study critical viscoelastic behavior. One of the most astonishing results is the excellent agreement of the Rouse exponent with the result $k=0.70$ from molecular dynamics simulations by Vernon et al [VePl01]. In these simulations the monomers are initially placed on a simple cubic lattice in the simulation volume and crosslinks are drawn with probability $p$ between nearest neighbors. This procedure ensures lattice percolation statistics for the connectivity. Crosslinks are modeled by harmonic springs. Even though these simulations do account for excluded volume interactions between monomers by a repulsive, soft-sphere potential, the result is nearly identical to the Rouse value for phantom clusters. A similar result, $k=0.65$, has been found by one of the latter authors in [JePl03] for a slightly different crosslinking mechanism. The influence of solvent was investigated in [PIVe03] by including uncrosslinked solvent particles explicitly in the MD simulations. A nonuniversal, concentration-dependent viscosity exponent $0.3<k<0.45$ was observed. It decreases with increasing density of the solvent.

In [JeP103] also the scaling $D_{n} \sim n^{-b_{D}}$ of the diffusion constant at the critical point has been obtained with $b_{D}=0.69$. A similar result was found in [Jes02]. It is worth mentioning, that the simulations in [JePl03] also verify the validity of a widely used scaling relation for the diffusion constant in terms of the radius of gyration in concentrated solutions, namely $D\left(R_{\text {gyr }}\right) \sim R_{\text {gyr }}^{-(1+k / \nu)}$ [Gen79b]. This is interesting insofar as the computation of the viscosity in another, quite different class of simulations by del Gado et al [GaAr00] is based on precisely this scaling relation. The latter simulations were done by means of the bond fluctuation method on a lattice and the scaling of the diffusion constant $D_{n} \sim n^{-b_{D}}$ is found to be given by $b_{D} \approx 1.0$. By means of the above scaling relation the viscosity exponent $k \approx 1.3$ is computed which is significantly higher than the result from the off-lattice MD simulations in [VeP101]. For the elastic modulus an exponent $\mu \approx 2.5$ was found from the lattice simulations [GaAr02].

Besides critical properties, also Brownian dynamics simulations of single hyperbranched polymers were performed by Sheridan et al [ShAd02]. They account for fluctuating hydrodynamic interactions corresponding to $\kappa=0.35$, as well as for excluded-volume 
interactions and lead to $b_{\eta}=0.13$. This result is remarkably close to the Zimm prediction $b_{\eta} \approx 0.11$ whereas the experimental findings on $b_{\eta}$ are consistently above the Zimm value.

Most striking, the experimental data scatters over a broad range and the exposition of a systematic trend is not possible. Concerning the viscoelastic exponents it is desirable that experiments would always measure at least two viscoelastic exponents. This would simplify the classification of the exponents. Some results agree quite well with the Rouse model whereas other exponents differ significantly. In order to judge this agreement and the quality of the predictions of the Rouse and Zimm model it is helpful to consider also existing theoretical (scaling) approaches to the gelation transition.

\subsection{Comparison to scaling theories}

\subsubsection{The electrical analogy}

Since the electrical analogy (see Sec. 1.3.2) is so widely employed as a "theory" of viscoelasticity it is interesting to reveal how the exact resistance mapping, which is used in this work, is related to it. The electrical analogy comprises two predictions, one for the viscosity and the other one for the shear modulus.

The first proposal $k=s$ of the electrical analogy [Gen78] (see also Sec. 1.3.2) for the viscosity relates its divergence to the divergence of the conductivity of a conductor/superconductor network. In contrast the exact mapping (cf. Sec. 5.2.1) relates the viscosity to the divergence of the average resistance in an insulator/conductor network. In fact, the exact mapping for the viscosity exponent $k$ in the Rouse model disproves the long standing scaling relation $k=s$. This can hardly be revealed by comparing the numerical values in $d=3, k \approx 0.71$ and $s \approx 0.75$ [HeDe84]. However, in $d=2$ duality implies $s=\phi[$ Str77] in terms of the crossover exponent $\phi$ of the random resistor network whereas the exact result for the viscosity is $k=\phi-\beta$ which is in contradiction with $k=s$. Note, that sometimes the scaling relation $s=\nu-\beta / 2$ [Ker83] is used which implies a similar numerical value $s \approx 0.67$ in $d=3$. However, this scaling relation is believed to be incorrect since it is equivalent to the Alexander-Orbach conjecture in $d=2$ [Ker83]. Alexander and Orbach conjectured [AlOr82] that the spectral dimension $d_{\mathrm{s}}=4 / 3$ of percolation clusters is independent of the spatial dimension for $2 \leq d \leq 6$. Although this conjecture has not yet been disproved rigorously, there is a large body of evidence against it (see [HaBe02] and references therein).

The second proposal of the electrical analogy relates the exponent $\mu$ of the shear modulus to the exponent $\tilde{s}$ of the vanishing conductivity in an insulator/conductor problem above $c_{\text {crit }}$ [Gen76a]. The conductivity exponent has been calculated by renormalization group methods up to second order in $\epsilon=(6-d)$ which yield the scaling relation $\tilde{s}=\phi+\nu(d-2)$ [LuWa85] in terms of the percolation exponent $\nu$ and the crossover exponent $\phi$. Unfortunately, the Rouse model as defined in Sec. 2.3.2 does not allow addressing the shear modulus and other quantities above the percolation transition since the gel phase requires the incorporation of stabilizing repulsive interactions. However, the shear modulus has been 
examined in a different model [XiMu04] which accounts for excluded volume interactions for stabilization. In this model phantom clusters are obtained by appropriately taking the limit of vanishing excluded volume strength. In this limit the second part of the electrical analogy can be derived, viz the exponent of the shear modulus equals the conductivity exponent $\mu=\phi+\nu(d-2)$. Most interesting in [XiMu04] it is claimed that in the phantom limit the field theory of the gelation transition becomes identical to the field theory of random resistor networks [HaLu87], and hence identical to the same system which has been employed here to compute the viscosity. Hence, both exponents can be computed from the same electrical insulator/conductor problem. Concerning the shear modulus this is in accordance with the long-standing scaling argument. Concerning the viscosity, the exact electrical analog differs from the long-standing phenomenological one, a fact which has also been suspected in [SaGo85].

It seems that the electrical analogy can be recovered from a gelling system by appropriate phantom limits, i.e. for subdominant excluded volume interactions. From some experimental results for the viscosity and the shear modulus and from the computer simulations [VePl01] one might also conjecture that the phantom limit is a suitable description for certain systems. Indeed this poses the fundamental question, if there is any justification for neglecting excluded volume interactions and applying a phantom description to gelling percolation clusters. Therefore, it will be useful to shed some light onto the relevance of excluded volume interactions in a system of percolation clusters.

\subsubsection{The relevance of excluded volume interactions}

Is it justifiable to neglect excluded volume interactions for percolation clusters under certain circumstances? This question is indeed motivated by the fact that for linear polymers the dense melt does provide such a circumstance where excluded volume interactions are screened and the phantom description is perfectly reasonable. In order to understand this screening of excluded volume interactions for linear polymers and address the above question at least phenomenologically, one can apply a so called Flory argument [Gen79a, DoEd88, RuCo03], which is reviewed in the following. A Flory argument estimates the relevance of excluded volume interactions for the size of a tagged, fractal, macromolecule which is immersed in a solution of other objects. These objects are allowed to be monomers, linear chains, macromolecules, fractals etc. The tagged macromolecule consists of $n$ monomers and it is characterized by its Gaussian fractal dimension $d_{\mathrm{f}}^{(\mathrm{G})}$, which is

an intrinsic property of the fractal relating its ideal radius $R_{0} \sim n^{d_{\mathrm{f}}^{(\mathrm{G})}}$ to its size $n$ in the of absence of excluded volume interactions. In the presence of excluded volume interactions the dependence of the fractal's (swollen) radius $R$ on the size is characterized by a different, smaller fractal dimension $d_{\mathrm{f}}$ which is to be determined now. Therefore, it is assumed [IsLu80, Vil88] that the free energy of the tagged fractal is given by the following simple form

$$
F(R) \sim\left(\frac{R}{R_{0}}\right)^{2}+v \frac{1}{n_{\mathrm{w}}} \frac{n^{2}}{R^{d}} .
$$


The first term estimates the entropic penalty for stretching the fractal to a radius $R$. The second term estimates the energetic penalty of $n$ monomers being uniformly distributed in the $d$-dimensional volume $R^{d}$, where $v$ is the strength of the excluded volume interactions. The fractals which surround the tagged one are characterized by their weight average molecular weight $n_{\mathrm{w}}$ which reflects the screening of the interactions due to the presence of other clusters inside the pervaded volume of the tagged cluster. It is assumed that both sizes can be related to each other by the scaling $n_{\mathrm{w}} \sim n^{\rho}$. The equilibrium extension is then obtained by minimizing $F(R)$ with respect to $R$. This yields the fractal dimension

$$
d_{\mathrm{f}}=\frac{d+2}{2-\rho+2 / d_{\mathrm{f}}^{(\mathrm{G})}}
$$

of the tagged cluster. In order to estimate the importance of excluded volume interactions the swelling ratio $r_{\text {swell }}:=R / R_{0} \sim n^{d_{\mathrm{f}}^{(\mathrm{G})}(2-\rho)-d}$ is examined. It is obvious that there is an upper critical dimension $d_{\mathrm{uc}}=d_{\mathrm{f}}^{(\mathrm{G})}(2-\rho)$ at which the swelling ratio $r_{\mathrm{swell}}$ becomes of order one and thus excluded volume interactions become irrelevant. The most important examples are given in the following.

If the tagged polymer is a linear chain then $d_{\mathrm{f}}^{(\mathrm{G})}=2$ i.e. the ideal random walk. If this chain is surrounded by small solvent particles, i.e. monomers one has $\rho=0$ and Eq. (7.2) predicts Flory's famous result for the fractal dimension of a swollen linear chain, $d_{\mathrm{f}}=(d+2) / 3$, which is valid below the upper critical dimension $d_{\mathrm{uc}}=4$. Next, if the surrounding monomers are replaced by linear chains of the same size one has $\rho=1$ and hence an upper critical dimension $d_{\mathrm{uc}}=2$. For $d \geq 2$ one has $d_{\mathrm{f}}=2$ which is nothing but the initially mentioned result that in 3-dimensional polymer melts excluded volume interactions are screened and the size of the tagged polymer is described by the ideal random walk.

Next, the tagged fractal is chosen as the characteristic percolation cluster of size $n=$ $n^{*}$. It suffices to employ the mean field percolation exponents which yield the Gaussian fractal dimension $d_{\mathrm{f}}^{(\mathrm{G})}=4$ and relate $n^{*}$ to the weight average molecular weight by $\rho=$ $1 / 2$. As a result, the Flory argument predicts $d_{\mathrm{uc}}=6$ for the upper critical dimension of excluded volume interactions for percolation clusters. The fractal dimension of three dimensional percolation clusters is then estimated to $d_{\mathrm{f}}=2.5$, which agrees very well with numerical value of the Hausdorff fractal dimension of lattice percolation clusters $d_{\mathrm{f}}^{(\mathrm{H})}=$ 2.53. If instead, the percolation clusters are immersed in a solvent of monomers, i.e. $\rho=0$ the excluded volume interactions become even more important. This is reflected by a shift of the upper critical dimension to $d_{\mathrm{uc}}=8$ below which the prediction $d_{\mathrm{f}}=2(d+2) / 5$ for the swollen fractal dimension of percolation clusters in dilute solutions is an excellent estimate of observed value the $d_{\mathrm{f}} \approx 2$ in $d=3$.

It is pointed out that the Flory argument solely considers static properties of fractals in equilibrium. The argument implies that three dimensional percolation clusters are always affected by excluded volume interactions as far as static, equilibrium properties are concerned. This holds true in the absence of a solvent and the effect is even more pronounced in the presence of a solvent. Unfortunately, if this argument is simply generalized to dy- 


\begin{tabular}{l||ccccc} 
& $k$ & $z$ & $\theta(=z \Delta)$ & $\Delta(=\theta /(k+\theta))$ & $\mu(=z \Delta)$ \\
\hline \hline $\operatorname{Rouse}\left(\nu^{(\mathrm{G})}\right)$ & $2 \nu^{(\mathrm{G})}-\beta$ & $2 \nu^{(\mathrm{G})}+1 / \sigma$ & $(\tau-1) / \sigma$ & $(\tau-1) /\left(2 \sigma \nu^{(\mathrm{G})}+1\right)$ & - \\
$\operatorname{Rouse}(\nu)$ & $2 \nu-\beta$ & $2 \nu+1 / \sigma$ & $d \nu$ & $d \nu /(2 \nu+1 / \sigma)$ & $d \nu$ \\
$\operatorname{Zimm}\left(\nu{ }^{(\mathrm{G})}\right)$ & $0^{1)}$ & $d \nu$ & - & - & - \\
$\operatorname{Zimm}(\nu)$ & $0^{2)}$ & $d \nu$ & $d \nu$ & 1 & $d \nu$
\end{tabular}

Table 7.4: Scaling predictions for the viscoelastic exponents. The results in this work are given by Rouse $\left(\nu^{(\mathrm{G})}\right)$ and ZIMM $\left(\nu^{(\mathrm{G})}\right)$ in terms of the Gaussian correlation length whereas Rouse $(\nu)$ and ZIMM $(\nu)$ refer to scaling arguments which involve the lattice percolation length. 1): fi nite viscosity. 2): logarithmic divergence.

namics in equilibrium which completely determines the regime of linear viscoelasticity one is forced to question the applicability of models which neglect excluded volume interactions. However, this is not necessarily true for systems under the influence of external stresses or in nonequilibrium situations. This is discussed as an open problem in Sec. 7.5

Moreover, the Flory argument is well suited for a sound understanding of how the results from the Rouse and the Zimm model are to be compared to existing scaling approaches since these scaling arguments relate the fundamental time scale to a fundamental length scale which is characterized by a fractal dimension (see Sec. 1.3.2).

\subsubsection{Viscoelasticity in the absence of hydrodynamic interactions}

Next, the exact Rouse exponents $k, \theta, \Delta, z$ of critical rheology are compared to the set exponents stemming from scaling arguments in the absence of hydrodynamic interactions as given in Sec. 1.3.2. These exponents are usually referred to as the Rouse exponents which is always meant as a synonym for "in the absence of hydrodynamic interactions" but with the ambiguity that it is sometimes used in the sense "in the absence of hydrodynamic interactions and in the presence of excluded volume interactions " and sometimes in the sense "in the absence of both, hydrodynamic and excluded volume interactions". It is exactly this difference which becomes important now.

As reviewed in Sec. 1.3.2, in the absence of hydrodynamic interactions the scaling arguments estimate the longest relaxation time $t^{*}=\xi^{2} / D_{n^{*}}$ within the assumption that the diffusion constant $D_{n^{*}}$ is inversely proportional to the size of the cluster which is, without a doubt, valid in the Rouse model. Then, the size $\xi$ of the largest cluster is estimated by the correlation length of lattice percolation, yielding $z=2 \nu+1 / \sigma$ for the critical exponent of the characteristic relaxation time. In contrast, the Rouse model, as defined here, describes phantom clusters which are governed by the Gaussian correlation length with an exponent $\nu^{(\mathrm{G})}$, yielding $z=2 \nu^{(\mathrm{G})}+1 / \sigma$ for the exponent of the characteristic time scale. Therefore it will be useful to label the exact Rouse exponents which are computed in this work in terms of the Gaussian correlation length by Rouse $\left(\nu^{(\mathrm{G})}\right)$ and the exponents from the scaling argument which involve the correlation length of lattice percolation by RouSE $(\nu)$. 
Two conclusion can be drawn by confronting the Rouse $\left(\nu^{(\mathrm{G})}\right)$ with the Rouse $(\nu)$ exponents: (i) The formal similarity of the exponents demonstrates that the scaling argument can also be applied to the Rouse model of phantom clusters if only the phantom nature is accounted for consistently by inserting the Gaussian correlation length as the characteristic length scale. Interpreted in this manner the scaling argument correctly resembles the viscosity exponent by replacing the correlation length exponent $\nu$ in the scaling prediction $k=2 \nu-\beta$ with the Gaussian correlation length $\nu^{(\mathrm{G})}$, yielding the exact result $k=2 \nu^{(\mathrm{G})}-\beta$. Likewise, it is applicable to $\Delta$ which can be computed from $k, z$ via the scaling relation $\Delta=(z-k) / z$. (ii) The ROUSE $(\nu)$ exponents do not describe a system where excluded volume interactions are irrelevant as is sometimes assumed incorrectly. The possible significance of the Rouse $(\nu)$ exponents is suggested from the Flory argument in the previous section: The exponent $\nu=1\left(\sigma d_{\mathrm{f}}^{(\mathrm{H})}\right)$ describes static properties of gelation clusters having percolation connectivity with excluded volume interactions and therefore the ROUSE $(\nu)$ exponents most likely describe critical rheological properties of percolation clusters with excluded volume interactions. This claim is, to some extent supported by renormalization group calculations [Wie98a] for the dynamics of self-avoiding membranes and in particular linear polymer chains in the absence of hydrodynamic interactions. The mean squared displacement of an arbitrary monomer grows with an exponent $2 / d_{\mathrm{f}}+1$ for large times. Thereby $d_{\mathrm{f}}$ is to be chosen as the Gaussian fractal dimension $d_{\mathrm{f}}=d_{\mathrm{f}}^{(\mathrm{G})}=2$ if excluded volume interactions are irrelevant, i.e. for $d>d_{\mathrm{uc}}=4$ whereas for $d<d_{\mathrm{uc}}$ the fractal dimension $d_{\mathrm{f}} \approx 1.70$ (see [DoEd88, Ch. 2.5]) of the excluded volume dominated, linear polymer chain is to be inserted.

There remain two viscoelastic exponents which have not been discussed yet with reference to the comparison between Rouse $\left(\nu^{(\mathrm{G})}\right)$ and Rouse $(\nu)$, the exponents $\theta$ and $\mu$ of the recoverable compliance $J$ and the shear modulus $G$, respectively. Both exponents $\mu$ and $\theta$ describe (shear) elastic properties, since the recoverable compliance can be regarded as an inverse shear modulus on large but finite time scales. Therfore, it is often argued [MaAd88, MaAd91, LuMo95, WiMo97] that the exponent $\mu$ which describes the vanishing of the static shear modulus above the gel point is identical to the exponent $\theta$ which describes the divergence of the recoverable compliance below the gel point. The scaling argument ROUSE $(\nu)$ predicts $G=k_{\mathrm{B}} T / \xi^{d}$ from dimensional analysis and hence $\mu=d \nu$ (see also Sec. 1.3.2). As a consequence both exponents of elasticity are given by $\mu=\theta=d \nu$. Since the ROUSE $(\nu)$ exponents are computed from lattice percolation hyperscaling must be valid. By employing the (hyper)scaling relations of percolation (cf. Tab. 1.1) $d \nu=(\tau-1) / \sigma$ is obtained for the exponents of the shear modulus and the recoverable compliance within Rouse $(\nu)$ [LuMo95], [CoGi93]. Most surprisingly, the same exponent $\theta=(\tau-1) / \sigma$ is also found for the exact Rouse $\left(\nu^{(\mathrm{G})}\right)$ value without referring to hyperscaling relations. In other words, the product $\theta=z \Delta$ assumes the same value $(\tau-1) / \sigma$ for both RouSE $\left(\nu^{(\mathrm{G})}\right)$ and RouSE $(\nu)$ which implies that $z \Delta$ is completely determined by the underlying cluster size distribution and is independent of the spatial arrangement.

The numerical predictions of the two exponent classes Rouse $\left(\nu^{(\mathrm{G})}\right)$ and Rouse $(\nu)$ in the absence of hydrodynamic interactions are highly different (see Tab. 7.2). This is 
obvious since the suggested relevance of excluded volume interactions for RouSE $(\nu)$ slow down the relaxation processes due to a larger extension of the clusters in space.

\subsubsection{Viscoelasticity in the presence of hydrodynamic interactions}

The label Zimm is always used as a synonym of dominant hydrodynamic interactions but it has the same ambiguity as the label Rouse: Sometimes it is used in the sense "phantom clusters in the presence of hydrodynamic interactions" and sometimes as "excluded volume dominated clusters in the presence of hydrodynamic interactions". Similar to the discussion of the scaling arguments for the Rouse model this ambivalence suggests to introduce the label ZIMm $\left(\nu^{(\mathrm{G})}\right)$ for the exponents obtained in this work which assume Gaussian clusters and the label ZIMM $(\nu)$ for scaling arguments which assume the length scale to be set by the correlation length of lattice percolation.

For ZIMM $\left(\nu^{(\mathrm{G})}\right)$ the scaling $z=3 \nu^{(\mathrm{G})}$ in $d=3$ for the typical relaxation time has been found. This is in accordance with the ZIMM $(\nu)$ proposal $z=d \nu$ [MaAd88] for unswollen percolation clusters in the presence of hydrodynamic interactions (cf. also Sec. 1.3.2). The scaling relation $z=d \nu$ is also corroborated by results from Wiese [Wie98b], who defined the dynamic exponent by the mean-squared displacement of a single monomer of a polymer chain in the presence of non-preaveraged hydrodynamic interactions. The result is translated to a time scale which is given by $t_{n} \sim R_{n}^{d}$ in terms of the radius of gyration. This is in fact the starting point for the scaling argument ZIMM $(\nu)$.

Turning to the viscosity first the Mark-Houwink relation $\eta_{n} \sim n^{b_{\eta}}$ is discussed. In [Mut85, Cat85] it was suggested that the Mark-Houwink exponent is given by $b_{\eta}=d / d_{\mathrm{f}}-$ 1 (see also Sec.1.3.2) irrespective of excluded volume interactions. Since phantom clusters are governed by the Gaussian fractal dimension $d_{f}=d_{\mathrm{f}}^{(\mathrm{G})} \approx 4$ the scaling relation implies $b_{\eta} \approx-1 / 4$ in $d=3$. This value is definitely ruled out by the numerical data. This disproves the validity of the scaling relation for for phantom clusters in the Zimm model.

If instead the scaling argument ZIMM $(\nu)$ is employed for the viscosity the fractal dimension of lattice percolation must be inserted which gives $b_{\eta}=d / d_{\mathrm{f}}^{(\mathrm{H})}-1$ [StCo82]. By weighting these contributions of clusters of size $n$ with the cluster size distribution the exponent $k=\left(1-\tau+d / d_{\mathrm{f}}^{(\mathrm{H})}\right) / \sigma$ is obtained for the shear viscosity for percolation clusters with fractal dimension $d_{\mathrm{f}}^{(\mathrm{H})}$. By employing the (hyper)scaling relations of percolation 1.1 one finds $k=\left(1-\tau+d / d_{\mathrm{f}}^{(\mathrm{H})}\right) / \sigma=0$ which is then attributed to a logarithmic divergence [StCo82]. From $k=0$ and $z=d \nu$ the other viscoelastic exponents can be obtained by the scaling relations of critical rheology which constitute the ZIMM $(\nu)$ values in Tab. 7.4.

A completely different scaling relation $k=\nu-\beta / 2$ for the viscosity exponent in the Zimm model was proposed in [ArSa90]. This proposal is also ruled out definitely by the numerical results.

The reason for the failure of the Zimm model in predicting a diverging viscosity can be traced back to a too slowly diverging typical relaxation time $t^{*} \sim \varepsilon^{z}$ in the Zimm model. This is due to the phantom nature of the clusters, where the monomers are in close proximity in a space. Thus, relaxations can occur on very small time scales which lead to a very 
weak divergence of the longest relaxation time with increasing cluster size. As a consequence the viscosity remains finite and, of course, in the absence of a divergence scaling theory fails. Thus, the inclusion of hydrodynamic interactions requires most certainly also the inclusion of excluded volume interactions which is then most likely recovered by the ZIMM $(\nu)$ exponents. The failure of the Zimm model for Gaussian percolation clusters is to be contrasted with the success for a solution of linear polymer chains where the Zimm model for the Gaussian chain yields a realistic description of viscoelasticity at the theta point (see [DoEd88, Ch. 4.5] and references therein).

The preaveraging approximation of hydrodynamic interactions is most likely not responsible for a finite viscosity and the unrealistic exponent values in the Zimm model. This is suggested, since linear polymers show a decrease in the viscosity when abandoning the preaveraging approximation [Fix81] and the effects of preaveraging for branched polymers are even more pronounced than for linear ones [BuSc80]. For linear polymers it is also known that only prefactors of the Mark-Houwink relation are diminished as a result of preaveraging while the scaling exponent $b_{\eta \text {,linear }}$ remains unchanged [OoKo83]. This holds true to first order in $\epsilon=(4-d)$ for both, the Gaussian chain and the self avoiding chain.

In contrast, another shortcoming which is most likely cured by abandoning the equilibrium preaveraging procedure is the vanishing second normal stress coefficient $\Psi^{(2)}=0$. The consistent preaveraging in the absence of excluded volume interactions yields a nonzero but positive second normal stress coefficient, $\Psi^{(2)}>0$ [Oet87a]. In order to obtain a negative second normal stress coefficient $\Psi^{(2)}<0$, as observed in experiments, the preaveraging has to be abandoned completely [Oet89, Wan89].

\subsubsection{Density fluctuations}

Turning to density fluctuations, the derived decomposition of the incoherent scattering function into single cluster exponentials which reflecting the diffusive motion of the clusters has also been suggested on phenomenological grounds [Gen79b, MaWi88].

In the Rouse model, the exponents $\alpha_{S}, \delta$ which characterize the long time decay of the incoherent scattering function for $t \rightarrow \infty$ are not compatible with existing scaling arguments. This failure can be traced back to the fact that scaling arguments are based on the Stokes-Einstein relation (cf. Eq. (1.14) in Sec. 1.3.2) for the diffusion constant whereas in Rouse model the averaged diffusion constant of a cluster does not obey a Stokes-Einstein scaling form in terms of the the averaged radius of gyration. This shortcoming stems from oversimplified picture that in the Rouse model diffusion constants are simply inversely proportional to the size of the cluster and do not depend on the shape at all.

In contrast the Zimm model does predict a scaling form $D_{n} \sim / R_{\mathrm{gyr}, n}$. The stretched exponential decay of the incoherent scattering function is governed by the exponent

$\alpha_{S}=d_{\mathrm{f}}^{(\mathrm{G})} /\left(d_{\mathrm{f}}^{(\mathrm{G})}+1\right)$ which is in accordance with scaling arguments [MaWi88] where an exponent $\alpha_{S}=d_{\mathrm{f}} /\left(d_{\mathrm{f}}+1\right)$ has been proposed by employing the Stokes-Einstein relation. 
The fact that in the Zimm model the diffusion constant is inversely proportional to the radius of gyration supports the hard-sphere or non-draining picture of Zimm clusters: In general, one can define a hydrodynamic radius of a cluster following Stokes law $D_{n}=: k_{\mathrm{B}} T /\left(6 \pi \eta R_{\mathrm{hyd}, n}\right)$. If the hydrodynamic radius scales with the same exponent as the radius of gyration then the cluster behaves effectively as a dense object which is not drained by flow, in agreement with Stokes law for hard spheres. For comparison, the Rouse model corresponds to the freely draining case. Despite being a good approximation, the non-draining picture is not valid strictly [Oet96b]. The hard sphere picture is not limited to preaveraged hydrodynamic interactions. Renormalization group calculations [Wie98b] indicate that even polymers with fluctuating hydrodynamic interactions have the same dynamic exponent.

For the nonlinear dynamic susceptibility $\chi(\boldsymbol{q}, t)$ the derived scaling form of the lower bound is of precisely that form one would anticipate within the generally accepted assumption of dynamic scaling [HoHa77] for a system with a characteristic length scale $\xi^{*}$ and time scale $t^{*}$.

\subsection{Conclusions}

In summary, the quantities, which do exhibit critical behaviour in the Rouse and in the Zimm model, correctly recover existing scaling arguments provided that the Gaussian fractal dimension is used.

The Gaussian nature of the percolation clusters i.e. the neglect of excluded volume interactions seems to be the crudest drawback of the present theory as suggested by the Flory argument. However, the Rouse model with three dimensional percolation statistics agrees quite well with some experiments, a fact which still lacks an explanation (this point is again considered in the next section).

In contrast the Zimm model has turned out to give an unrealistic picture of the gelation transition. The failure of predicting a diverging viscosity and the poor agreement of other quantities with experiments are most likely due to the presence of hydrodynamic interactions in the absence of excluded volume interactions.

Another drawback of the theory is that entanglement effects are neglected. These topological interactions (see [DoEd88, Ch. 5.4]) are argued to play a vital role in stress relaxation. However, temporary entanglements are expected to play a minor role [CoGi93] in the dynamical regime on the longest time scales close to the transition since the time scale of a temporary entanglement is determined by the smaller cluster whereas the dynamics on the longest time scales is determined by the largest clusters. Permanent entanglement effects due to interlocking loops are clearly beyond the scope of this work. 


\subsection{Open problems}

A possible explanation of the good agreement of some experimental values with the Rouse exponents is the existence of a crossover from phantom behaviour to excluded volume dominated behaviour. In fact, the aforementioned result [XiMu04] for the shear modulus (see Sec. 7.3.3) predicts exactly such a crossover from the phantom limit, which is governed by the conductivity exponent $\mu=\phi+\nu(d-2)$, to a different regime which is governed by the exponent $\mu=d \nu$. In order to shed some light onto this crossover and its possible conclusions for the dynamics in the presence of excluded volume interactions it is helpful to review briefly the characteristics of the model employed in [XiMu04]. This provides a suitable conceptual framework for further discussions.

The model is a generalization of the statistical mechanics approach to static properties of gelation from [GoCa96]. It describes a system of identical polymers consisting of a finite number of monomers which interact via excluded volume interactions. Prior to crosslinking the state of the system is referred to as preparation state. The preparation state is characterized by the Edwards-Hamiltonian which accounts for the connectivity of each individual polymer chain and the mutual excluded volume interactions between all monomers in the system. The excluded volume interactions determine the compressibility $B_{0}$ of the system in the preparation state. Then, the polymers are to be connected by hard crosslinks. After crosslinking the state of the system is referred to as measurement state and is characterized by a Hamiltonian which accounts for the connectivity of the chains and excluded volume interactions which are allowed to differ in strength from that of the preparation state. Accordingly, the measurement state is characterized by a compressibility $B$ which may be different from $B_{0}$. This distinction between preparation state and measurement state emerges naturally in this model as a consequence of the Deam-Edwards distribution which is used for the crosslink statistics: This distribution is based on the idea, that the equilibrium configurations of polymers in the preparation state without crosslinks determine the probability of the crosslinks such that, at the bottom of the line, crosslinks are more likely drawn between monomers which are in close proximity in the preparation state.

The special case $B_{0}=B \neq 0$, i.e. identical excluded volume strengths in the preparation and measurement state has been discussed in a series of papers. The most important for this discussion is the renormalization group approach [PeGo00] which shows that the Deam-Edward distribution recovers the geometric properties of lattice percolation for $B_{0}=B \neq 0$ (see also [JaSt01]). This seems plausible, since nonvanishing excluded volume interactions during preparation causes spatial correlations with an averaged number of nearest neighbors which is similar to that of the lattice system. The situation is similar to continuum (off-lattice) percolation which is also expected to be in the same universality class as lattice percolation.

If the shear modulus is computed for $B_{0}=B \neq 0$ the critical exponent $\mu=d \nu$ is obtained [CaGo00]. The other case which has been studied in [XiMu04] is $B_{0} \gg B$. Although, both compressibilities are expected to be large in real systems so that the density essentially remains constant throughout the transition they can still differ by a large factor. 
In the idealized case, when $B$ vanishes the shear modulus is governed by the exponent $\mu=(d-2) \nu+\phi$ of the conductivity of the insulator/conductor percolation problem to first order in $(d-6)$.

Indeed, in real systems the ratio $B / B_{0}$ might be small but never zero. Then it is predicted [XiMu04] that the shear modulus exponent should crossover from its phantom behaviour $\mu=\phi+\nu(d-2)$ to the excluded volume dominated behaviour $\mu=d \nu$ since the latter is governed by a more stable fixed point of the RG analysis. The emergence of the conductivity exponent of percolation clusters gives strong evidence that a nonvanishing $B_{0}$ still ensures that the Deam-Edwards distribution recovers percolation statistics in the preparation state. A vanishing $B$ then ensures a phantom description of these percolation clusters in the measurements state. The dynamical description of this state in the sol phase is expected to be given by the Rouse model as it is adopted here: The Rouse model assumes percolation statistics without referring to a preparation state. This can be regarded as if the crosslinks were generated in a preparation state with excluded volume interactions $B_{0} \neq 0$. The Rouse model then describes the dynamics of phantom percolation clusters which can be regarded as if excluded volume interactions being switched off in the measurement state, i.e. $B=0$. If the shear modulus crosses over from the excluded volume dominated value $\mu=d \nu$ to the value $\mu=\phi+\nu(d-2)$ in the phantom limit one might conjecture that, the viscosity exponent is also affected by this limit and should cross over from the still unknown excluded volume dominated value (presumably $k=2 \nu-\beta$ ) to its phantom value $k=\phi-\beta$. This suggests that both viscoelastic exponents $k=\phi-\beta$ and $\mu=\phi+\nu(d-2)$ can be derived from the problem of gelling percolation clusters in the limit of subdominant excluded volume interactions in the measurement state which seems to be equivalent to the electrical insulator/conductor problem.

This crossover, which describes the relevance of excluded volume interactions for fixed (percolation-like) connectivity is qualitatively different from another, well known crossover which can arise in gelation: The connectivity crossover from mean field percolation to $3 d$ percolation for the vulcanization of long polymer chains. This connectivity crossover is easily illustrated by the above picture: If the preparation state comprises long precursor chains in a dense melt then these chains are ideal according to the above Flory argument. This pertains to a small value $B_{0} \approx 0$. In the picture of the Deam-Edwards distribution, a small $B_{0}$ in the preparation state leads then to many nearest neighbors during crosslinking and one monomer could be connected to many others. Thus, a mean field like connectivity is generated. This sensitivity of the nature of the Deam-Edwards distribution on the strength of the excluded volume interactions in the preparation state is supported by unpublished results: If crosslinks are chosen according to the Deam-Edwards distribution in the absence of excluded volume interactions in the preparation state, the shear viscosity can be computed and is identically given by the result (6.3) which was computed for the mean field like connectivity of Erdős-Rényi random graphs.

The above discussion suggest, that actually two crossovers may may play a vital role for the gelation transition in the absence of hydrodynamic interactions, a connectivity crossover from mean field percolation to $3 d$ percolation and a crossover from phantom clusters to excluded volume dominated clusters. Both features of the theory have the same 
upper critical dimension $d_{\mathrm{uc}}=6$. The above Flory argument also suggest, that if one includes a solvent the upper critical dimension for excluded volume should increase to $d_{\mathrm{uc}}=8$.

If both crossovers are observable in real systems the interplay of these crossovers might result in all combinations of connectivity (mean field or $3 d$ ) with bulk compressibilities (phantom or excluded volume dominated). This might be a reason why even in the absence of hydrodynamic interactions exponents are scattered over a broad range lying between $k=0$ (logarithmic divergence of phantom clusters with mean field connectivity) to $k \approx 1.3$ which is, presumably, the value for excluded volume dominated clusters with $3 d$ percolation connectivity.

The origin of the connectivity crossover is well understood, it can be driven by the length of the precursor chains [Gen77]. In contrast the possible physical origin for the crossover from phantom to excluded volume dominated behaviour is unclear. In [XiMu04] it is suggested that different qualities of solvent or correlations built up during the course of the chemical reaction might be responsible for a large ratio $B_{0} / B$. Another origin of phantom behaviour and a conductivity like scaling might be internal stresses [Ale84]. Alexander argued that the origin of shear rigidity stems basically from the crosslink interactions in the tenuous, macroscopic cluster while the compressibility of the system is maintained by the excluded volume interactions of all monomers in the system. Therefore, the macroscopic cluster can be regarded as a system under external stress (pressure) which is produced by the sol clusters. Then it is demonstrated in [Ale84] that in the presence of large stresses, the elasticity is dominated by the so-called scalar elasticity which is equivalent to the conductivity of the network [TaTh87, ZhLa00]. The influence of the stress $S$ on the critical behaviour is due to the introduction of a new length scale $\xi_{S}^{d-1}=k_{\mathrm{B}} T / S$ besides the correlation length. But, if the elasticity of the tenuous, macroscopic cluster in the gel phase is well described by scalar elasticity which seems to correspond to the phantom limit of gelation clusters [XiMu04] one might conclude that the presence of stresses also affect the viscoelastic properties of nearly macroscopic clusters in the sol phase. This conclusion is substantiated by the fact that, at least in the critical region and in the limit of linear response, the elastic and viscous properties are related to each other by a response function, the shear relaxation function $G(t)$, and thus by the Kramers-Kronig relations.

Beyond linear response, another interesting mechanism has been found which suppresses excluded volume interactions in a linear polymer chain, namely a simple shear flow with moderate shear rate $\dot{\gamma}$ [RaKa89]. If the chain is subjected to shear then the shear rate can be chosen small enough to avoid the the coil-stretch transition (see e.g. [Gen79a]) and at the same time large when compared to the inverse of the typical relaxation time $t_{n}$ of the chain. In this regime the conformation is affected considerably by the shear but the overall extension of the chain is still small when compared to the fully stretched state which ensures the Edwards- Hamiltonian to be a reasonable model. Note, that if the inverse shear rate becomes a relevant time scale it also introduces a new length scale via $\xi_{\dot{\gamma}}^{2} \sim k_{\mathrm{B}} T /\left(\dot{\gamma} \zeta_{n}\right)$. Alternatively the system can be regarded as being subjected to a stress $S \sim\left(k_{\mathrm{B}} T / R_{n}^{d}\right)\left(\dot{\gamma} t_{n}\right)$. This demonstrates that this stress is important only if the shear rate is of order of the inverse relaxation time $t_{n}$ of the chain. This effect might play a vital role 
in experiments system where it is increasingly difficult to guarantee the condition $\dot{\gamma} t_{n} \ll 1$ for steady state behaviour due to diverging relaxation times.

In order to complete the issue of crossovers it is noted, that besides the elasticity crossover from $\mu=\phi+\nu(d-2)$ to $\mu=d \nu$ which is found in [XiMu04], there is another crossover for the elasticity problem in disordered solids which has generated considerable interest in the last decades (see [NaYa94, Sec. V A.3]). This is the crossover from scalar elasticity governed by $\mu=\phi+\nu(d-2)$ to regime of so-called vectorial elasticity [KaWe84] which is governed by the exponent exponent $\mu=d \nu+1$. This type of elasticity is modeled by introducing bond bending forces in a diluted percolation lattice. Bond bending forces intend to model local rigidity of the amorphous solid, which is less important for the sparsely connected gel in the vicinity of the transition. However, this crossover has also been observed experimentally [DeBo93] well outside the critical region in the gel phase.

To summarize, it is inevitable to study dynamics of gelling systems with excluded volume interactions. Furthermore it seems necessary to examine the role of initial stresses on the viscoelastic response.

\subsection{Outlook}

A first step towards computing the viscosity with excluded volume can be done. Following Fixman [Fix83a] (see also [DoEd88, Ch. 4.6]) one can derive lower and upper variational bounds for the viscosity and the diffusion constant in the presence or absence of hydrodynamic interactions in terms of equilibrium averages. In the absence of hydrodynamic interactions the bound for the viscosity can be derived by linearizing the Smoluchowski equation [DoEd88, Ch. 4.6] for small shear rates. It is given by (see App. I)

$$
\eta \geq \frac{\zeta}{2 d}\left\langle\sum_{k=1}^{K} \frac{N_{k}}{N} R_{\mathrm{gyr}}^{2}\left(\mathscr{N}_{k}\right)\right\rangle_{\mathrm{eq}} .
$$

The equilibrium expectation value in (7.3) involves the Boltzmann weight of a Hamiltonian with excluded volume interactions. In the absence of excluded volume interactions this bound coincides with the exact expression (cf. Eq. (4.37) and (5.19)). In the presence of excluded volume interactions this bound is most likely tractable by employing the results from the statistical mechanics approach with the Deam Edwards distribution for $B=B_{0} \neq 0$ (see above). For this choice of parameters the geometrical properties of the crosslinked gelation clusters lie in the percolation universality class [PeGo00]. Thus, one might compute the bound directly from percolation which gives $k=2 \nu-\beta$ [StAh95, $\mathbf{p}$. 65]. This is nothing but the result which is obtained from the scaling argument RoUSE $(\nu)$. The result indicates that in the presence of excluded volume interactions the viscosity exponent is at least $k>2 \nu-\beta$.

Unfortunately, the expression (7.3) cannot be inferred directly from the renomalization group calculations for the gelation transition since the quantity cannot be expressed simply in terms of the density fluctuations $\rho(x)$ of the overall monomer density. It requires rather 
the knowledge of density fluctuations of clusters of fixed size $n$. Alternatively, one may introduce an appropriate generating function. This can be seen by rewriting the bound (7.3) in terms of the projector $E_{0}$ as

$$
\eta \geq \frac{\zeta}{2 d} \frac{1}{N}\left\langle\sum_{i, j=1}^{N}\left[\mathbb{1}-E_{0}\right]_{i, j} \boldsymbol{R}_{i} \cdot \boldsymbol{R}_{j}\right\rangle_{\mathrm{eq}}
$$

An appropriate generating function is then given by

$$
\Xi(\lambda, \omega):=\left\langle\exp \left\{\lambda \sum_{i, j=1}^{N} \boldsymbol{R}_{i} \cdot \boldsymbol{R}_{j}[\mathbb{1}-\omega R(\omega)]_{i, j}\right\}\right\rangle_{\mathrm{eq}}
$$

in terms of the resolvent of $\Gamma$. The bound can be computed from $\Xi(\lambda, \omega)$ via

$$
\eta \geq \frac{\zeta}{2 d}\left\langle\left.\frac{1}{N} \lim _{\omega \downarrow 0} \frac{\partial}{\partial \lambda}\right|_{\lambda=0} \Xi(\lambda, \omega)\right\rangle
$$

the computation of which is left for future work.

In the Rouse model static and dynamic quantities are completely determined by the connectivity matrix. In more realistic models also other interactions play a role. Nevertheless, it might be interesting to explore the relationship between the static and dynamic properties and the spectral properties of the underlying connectivity matrix also in a more realistic model of gelation. Therefore one might investigate the spectral properties of the random connectivity matrix which is characterized by the Deam-Edwards distribution. This problem is somehow reminiscent of recent developments for glassy systems by means of so called Euclidean random matrix theory [CiGr03, GrMa03] which aim at the characterization of the vibrational density of states. Particles are localized per hand at random positions in space and the density of states of the Hessian from the harmonic expansion of the energy around these randomly chosen fixed positions is computed. The gelling system has the advantage that the random localization of particles is intrinsically contained in the model as a consequence of the properties of the underlying connectivity matrix. It would be interesting to study the connection between the vibrational density of states of randomly crosslinked amorphous solids, and the density of states of the underlying connectivity matrix, in particular, the relevance of the expected (spectral) localization transition of the connectivity matrix for the thermodynamic properties of the gelling system. 


\section{Appendix A}

\section{The equation of motion}

Here, following [OeRa89], the derivation of the equation of motion (2.11) is presented in a greater detail by starting from the coupled Langevin equations

$$
\begin{array}{r}
0=-\zeta\left(\frac{\mathrm{d}}{\mathrm{d} t} \boldsymbol{R}_{i}(t)-\boldsymbol{u}\left(\boldsymbol{R}_{i}, t\right)\right)+\boldsymbol{F}_{i}(t)+\boldsymbol{\xi}_{i}(t) \\
\rho_{\mathrm{s}}\left[\frac{\partial \boldsymbol{u}(\boldsymbol{r}, t)}{\partial t}+\boldsymbol{u}(\boldsymbol{r}, t) \nabla \boldsymbol{u}(\boldsymbol{r}, t)\right]=\eta_{\mathrm{s}} \Delta \boldsymbol{u}(\boldsymbol{r}, t)-\nabla p(\boldsymbol{r}, \boldsymbol{t}) \\
+\sum_{i=1}^{N} \boldsymbol{F}_{i}(t) \delta\left(\boldsymbol{r}-\boldsymbol{R}_{i}(t)\right)+\boldsymbol{f}(\boldsymbol{r}, t)
\end{array}
$$

for the monomer positions $\boldsymbol{R}_{i}(t)$ and the velocity field $\boldsymbol{u}(\boldsymbol{r}, t)$ subjected to the incompressibility condition

$$
\nabla \cdot \boldsymbol{u}(\boldsymbol{r}, t)=0
$$

The zero mean, Gaussian white noises $\boldsymbol{\xi}_{i}(t)$ and $\boldsymbol{f}(\boldsymbol{r}, t)$ are characterized by their second moments

$$
\begin{gathered}
\overline{\boldsymbol{\xi}_{i}(t) \boldsymbol{\xi}_{j}\left(t^{\prime}\right)}=2 k_{\mathrm{B}} T \zeta \delta_{i, j} \delta\left(t-t^{\prime}\right) \mathbf{1} . \\
\overline{\boldsymbol{f}(\boldsymbol{r}, t) \boldsymbol{f}\left(\boldsymbol{r}^{\prime}, t^{\prime}\right)}=-2 k_{\mathrm{B}} T \eta_{\mathrm{s}} \Delta \delta\left(\boldsymbol{r}-\boldsymbol{r}^{\prime}\right) \delta\left(t-t^{\prime}\right) \mathbf{1}
\end{gathered}
$$

Here, $\eta_{\mathrm{s}}$ and $\rho_{\mathrm{s}}$ are the solvent viscosity and density respectively and $\Delta$ denotes the Laplacian operator.

For highly viscous, i.e. small Reynolds numbers flows the convection term $\boldsymbol{u}(\boldsymbol{r}, t) \nabla \boldsymbol{u}(\boldsymbol{r}, t)$ in (A.2) is neglected. The resulting linear equation can be solved by introducing the spatial Fourier transform of the velocity field

$$
\widetilde{\boldsymbol{u}}(\boldsymbol{k}, t):=\frac{1}{(2 \pi)^{3}} \int_{\mathbb{R}^{3}} \mathrm{~d}^{3} r \boldsymbol{u}(\boldsymbol{r}, t) \mathrm{e}^{\mathrm{i} \boldsymbol{k} \cdot \boldsymbol{r}} .
$$

Eq. (A.2) is transformed into the ordinary differential equation

$$
\frac{\partial}{\partial t} \widetilde{\boldsymbol{u}}(\boldsymbol{k}, t)=-\frac{1}{\tau(k)} \widetilde{\boldsymbol{u}}(\boldsymbol{k}, t)-\frac{\mathrm{i}}{\rho_{\mathrm{s}}} \widetilde{p}(\boldsymbol{k}, t) \boldsymbol{k}+\frac{1}{\rho_{\mathrm{S}}(2 \pi)^{3}} \sum_{i=1}^{N} \boldsymbol{F}_{i}(t) \mathrm{e}^{\mathrm{i} \boldsymbol{k} \cdot \boldsymbol{R}_{i}(t)}+\frac{1}{\rho_{\mathrm{S}}} \widetilde{\boldsymbol{f}}(\boldsymbol{k}, t) .
$$


Here the solvent relaxation times are defined by $\tau(k):=\rho_{\mathrm{s}} /\left(\eta_{\mathrm{s}} k^{2}\right)$ with $k=|\boldsymbol{k}|$. If the transverse projection operator $\boldsymbol{P}_{\boldsymbol{k}}:=\mathbf{1}-\hat{\boldsymbol{k}} \hat{\boldsymbol{k}}^{\dagger}$ is applied to Eq. (A.7) the pressure term drops out whereas the incompressibility condition (A.3) amounts to $\boldsymbol{P}_{\boldsymbol{k}} \widetilde{\boldsymbol{u}}(\boldsymbol{k}, t)=\widetilde{\boldsymbol{u}}(\boldsymbol{k}, t)$. By integrating the resulting equation from initial time $t_{0}$ to $t$ one has

$$
\begin{aligned}
\widetilde{\boldsymbol{u}}(\boldsymbol{k}, t)= & \widetilde{\boldsymbol{u}}\left(\boldsymbol{k}, t_{0}\right) \mathrm{e}^{-\left(t-t_{0}\right) / \tau(k)}+\widetilde{\boldsymbol{u}}_{f}(\boldsymbol{k}, t) \\
& +\frac{1}{\rho_{\mathrm{s}}(2 \pi)^{3}} \sum_{i=1}^{N} \int_{t_{0}}^{t} \mathrm{~d} t^{\prime} \boldsymbol{P}_{\boldsymbol{k}} \boldsymbol{F}_{i}\left(t^{\prime}\right) \mathrm{e}^{\mathrm{i} \boldsymbol{k} \cdot \boldsymbol{R}_{i}\left(t^{\prime}\right)-\left(t-t^{\prime}\right) / \tau(k)}
\end{aligned}
$$

with a zero mean Gaussian white noise

$$
\widetilde{\boldsymbol{u}}_{f}(\boldsymbol{k}, t)=\frac{1}{\rho_{\mathrm{s}}} \int_{t_{0}}^{t} \mathrm{~d} t^{\prime} \boldsymbol{P}_{\boldsymbol{k}} \widetilde{\boldsymbol{f}}\left(\boldsymbol{k}, t^{\prime}\right) \mathrm{e}^{-\left(t-t^{\prime}\right) / \tau(k)} .
$$

The second moment of the real-space counterpart of (A.9) can be computed by inverse Fourier transformation of $\overline{\widetilde{\boldsymbol{u}}_{f}(\boldsymbol{k}, t) \widetilde{\boldsymbol{u}}_{f}^{\dagger}\left(\boldsymbol{k}^{\prime}, t^{\prime}\right)}$ which in turn employs the Fourier transformation of (A.5). In the limit $t_{0} \rightarrow-\infty$ it is given by

$$
\overline{\boldsymbol{u}_{f}(\boldsymbol{r}, t) \boldsymbol{u}_{f}\left(\boldsymbol{r}^{\prime}, t^{\prime}\right)^{\dagger}}=\frac{k_{\mathrm{B}} T}{\rho_{\mathrm{s}}(2 \pi)^{3}} \int_{\mathbb{R}^{3}} \mathrm{~d}^{3} k \boldsymbol{P}_{\boldsymbol{k}} \mathrm{e}^{\mathrm{i} \boldsymbol{k} \cdot\left(\boldsymbol{r}-\boldsymbol{r}^{\prime}\right)-\left|t-t^{\prime}\right| / \tau(k)}
$$

To proceed, the Markovian approximation is employed: The solvent relaxation times $\tau(k)$ are supposed to be small, so that the exponential $\exp \left\{\left|t-t^{\prime}\right| / \tau(k)\right\}$ is approximated by $2 \tau(k) \delta\left(t-t^{\prime}\right)$. As a consequence the second moment (A.10) simplifies to

$$
\overline{\boldsymbol{u}_{f}(\boldsymbol{r}, t) \boldsymbol{u}_{f}\left(\boldsymbol{r}^{\prime}, t^{\prime}\right)}=2 k_{\mathrm{B}} T \boldsymbol{\Omega}\left(\boldsymbol{r}-\boldsymbol{r}^{\prime}\right) \delta\left(t-t^{\prime}\right)
$$

with the Oseen tensor

$$
\begin{aligned}
\boldsymbol{\Omega}(\boldsymbol{r}) & =\frac{1}{\rho_{\mathrm{s}}(2 \pi)^{3}} \int_{\mathbb{R}^{3}} \mathrm{~d}^{3} k \frac{\rho_{\mathrm{s}}}{\eta_{\mathrm{s}} k^{2}}\left(\mathbf{1}-\hat{\boldsymbol{k}} \hat{\boldsymbol{k}}^{\dagger}\right) \mathrm{e}^{\mathrm{i} \boldsymbol{k} \cdot \boldsymbol{r}} \\
& =\frac{1}{8 \pi \eta_{\mathrm{s}} r}\left(\mathbf{1}+\hat{\boldsymbol{r}} \hat{\boldsymbol{r}}^{\dagger}\right) .
\end{aligned}
$$

The integral in (A.12) is computed in App. 3.III in [DoEd88]. By employing the Markovian approximation in (A.8) and neglecting the initial condition for $t_{0} \rightarrow-\infty$ the inverse Fourier transform can be carried out and yields

$$
\boldsymbol{u}(\boldsymbol{r}, t)=\boldsymbol{u}_{f}(\boldsymbol{r}, t)+\sum_{i=1}^{N} \boldsymbol{\Omega}\left(\boldsymbol{r}-\boldsymbol{R}_{i}(t)\right) \boldsymbol{F}_{i}\left(t^{\prime}\right)
$$

for the position dependent velocity field of the solvent in the presence of the particles at positions $\boldsymbol{R}_{i}(t)$. 


\section{Appendix B}

\section{The connectivity matrices}

In this appendix some properties of the connectivity matrices $\Gamma$ and $\widetilde{\Gamma}$ are summarized. Here, only single connected graphs (clusters) are considered. The generalization to the connectivity matrices for a collection of clusters is then easily accomplished by the decomposition of the connectivity matrix into clusters according to (2.19).

\section{B.1 Spectral properties of the graph Laplacian}

The connectivity matrix or graph Laplacian $\Gamma$ of an arbitrary connected graph with $n$ vertices is, by virtue of its definition (2.15), a symmetric, positive-semidefinite matrix. It can be written as the difference of the diagonal matrix of vertex degrees and the adjacency matrix of the graph

$$
\Gamma_{i, j}=\delta_{i, j} d_{i}-A_{i, j}
$$

By definition, the vertex degree $d_{i}$ of vertex $i$ is equal to the number of sites adjacent to $i$, i.e. $d_{i}=\sum_{j=1}^{n} A_{i, j}$ and hence the constant vector $\psi^{(0)}=n^{-1 / 2}(1,1, \ldots 1)$ is immediately identified as a normalized eigenvector with corresponding eigenvalue $\gamma_{0}=0$. It is non-degenerate [GoRo01, Ch. 13.1]. By labelling the eigenvalues according to $\gamma_{0}, \gamma_{1}, \ldots \gamma_{n-1}=0$ with $\gamma_{i}>0$ for $i=1 \ldots n-1$ and denoting the associated orthogonal eigenprojectors by $E_{i}$, the spectral decomposition is given by

$$
\Gamma=\sum_{i=1}^{n-1} \gamma_{i} E_{i}, \quad \text { with } E_{i} E_{j}=\delta_{i, j} E_{i} .
$$

The eigenprojectors corresponding to positive eigenvalues depend on the topology of the cluster. In contrast, the matrix elements of the eigenprojector $\left[E_{0}\right]_{i, j}=\psi_{i}^{(0)} \psi_{j}^{(0)}=1 / n$ depend only on the number of vertices in the cluster. Likewise, if a graph $\mathscr{G}$ has $K$ connected components (clusters) the nullspace can be decomposed according to

$$
E_{0}(\mathscr{G})=\bigoplus_{k=1}^{K} E_{0}\left(\mathscr{N}_{k}\right)
$$


and hence

$$
\left[E_{0}\right]_{i, j}=\frac{1}{N(i)} \delta_{\mathscr{N}(i), \mathscr{N}(j)}
$$

where the Kronecker delta is one if the cluster $\mathscr{N}(i)$ of particle $i$ is identical with the cluster $\mathscr{N}(j)$ of particle $j$ and zero otherwise.

The resolvent of $\Gamma$ is defined by $R(\omega):=(\Gamma+\omega \mathbb{1})^{-1}$ for $\omega \in \mathbb{C}$ and its spectral decomposition

$$
R(\omega)=\sum_{i=0}^{n-1} \frac{1}{\gamma_{i}+\omega} E_{i} .
$$

is a direct consequence of (B.2). For small $\omega$ the resolvent has an asymptotic expansion

$$
R(\omega)=\frac{1}{\omega} E_{0}+\sum_{i=1}^{N-1} \frac{1}{\gamma_{i}} E_{i}+\mathscr{O}(\omega) .
$$

The singular $\mathscr{O}\left(\omega^{-1}\right)$ term stems from the nullspace and the $\mathscr{O}(1)$ term is given by the Moore-Penrose inverse [Alb72] of $\Gamma$

$$
\frac{\mathbb{1}-E_{0}}{\Gamma}:=\sum_{i=1}^{n-1} \frac{1}{\gamma_{i}} E_{i},
$$

it is the inverse of $\Gamma$ restricted on the subspace of nonzero eigenvalues which is uniquely defined even for singular matrices. The Moore-Penrose inverse of $\Gamma$ is uniquely determined by

$$
\frac{\mathbb{1}-E_{0}}{\Gamma} \Gamma=\Gamma \frac{\mathbb{1}-E_{0}}{\Gamma}=\mathbb{1}-E_{0}
$$

\section{B.2 Relation between a symmetric or nonsymmetric, dy- namic matrix in the Zimm model}

Here, the relations between the spectral properties of different dynamic matrices in the Zimm model are shown, namely, the symmetric matrix $\widetilde{\Gamma}=H^{\frac{1}{2}} \Gamma H^{\frac{1}{2}}$ and the nonsymmetric matrix $\widehat{\Gamma}=H \Gamma$.

Let $H>0$ be a real, symmetric, positive-definite matrix of order $n$ and $\Gamma \geq 0$ a symmetric matrix characterized by its spectral decomposition $(\bar{B} .2)$. Then the matrix $\widetilde{\Gamma}:=$ $H^{\frac{1}{2}} \Gamma H^{\frac{1}{2}}$ is a real, symmetric matrix which is nonnegative and has a spectral decomposition

$$
\widetilde{\Gamma}=\sum_{i=1}^{n-1} \widetilde{\gamma}_{i} \widetilde{E}_{i}, \quad \widetilde{E}_{i} \widetilde{E}_{j}=\delta_{i, j} \widetilde{E}_{i} .
$$

The orthogonal projector $\widetilde{E}_{0}$ onto the nullspace of $\widetilde{\Gamma}$ is given explicitly in terms of the projector onto the nullspace of $\Gamma$ via

$$
\widetilde{E}_{0}=\frac{H^{-1 / 2} E_{0} H^{-1 / 2}}{\operatorname{Tr}\left(H^{-1} E_{0}\right)} .
$$


where $\operatorname{Tr}(\bullet)$ denotes the trace of a matrix. The normalization stems from the requirement $\widetilde{E}_{0} \widetilde{E}_{0}=\widetilde{E}_{0}$, which is easily shown by using $\left[E_{0}\right]_{i, j}=\psi_{i}^{(0)} \psi_{j}^{(0)}=1 / n$.

Let then $\widetilde{Q}$ be the orthogonal matrix which diagonalizes $\widetilde{\Gamma}$, viz

$$
\widetilde{Q}^{\dagger} \widetilde{\Gamma} \widetilde{Q}=\operatorname{diag}(\widetilde{\Gamma})
$$

where $\operatorname{diag}(\widetilde{\Gamma})$ is the diagonal matrix of eigenvalues of $\widetilde{\Gamma}$. Defining nonsymmetric matrices by $\widehat{\Gamma}:=H \Gamma$ and $\widehat{Q}:=H^{\frac{1}{2}} \widetilde{Q}$, it is deduced from $(\overline{\mathrm{B} .11})$ that

$$
\widehat{Q}^{-1} \widehat{\Gamma} \widehat{Q}=\operatorname{diag}(\widetilde{\Gamma}) .
$$

This proves, i) the nonsymmetric matrix $\widehat{\Gamma}$ is diagonalizable by the transformation $\widehat{Q}$ and ii) its real eigenvalues are equal to the eigenvalues of $\widetilde{\Gamma}$.

Denoting by $\widetilde{\psi}^{(n)}$ the eigenvector of $\widetilde{\Gamma}$ with corresponding eigenvalue $\widetilde{\gamma}_{n}$ such that $\widetilde{\Gamma} \widetilde{\psi}^{(n)}=\widetilde{\gamma}_{n} \widetilde{\psi}^{(n)}$ for $n=0 \ldots n-1$. Then $H^{-1 / 2} \widetilde{\psi}^{(n)}$ and $H^{1 / 2} \widetilde{\psi}^{(n)}$ are the left and right eigenvectors of $\widehat{\Gamma}$, respectively.

\section{B.3 Consistency condition for the Zimm model}

The consistency condition (4.30) in the Zimm model states that in the limit $t \rightarrow \infty$ the same equilibrium state is approached for Rouse and Zimm dynamics. Therefore, let

$$
\Gamma^{+}:=\left(\mathbb{1}-E_{0}\right) H^{\frac{1}{2}} \frac{\mathbb{1}-\widetilde{E}_{0}}{\widetilde{\Gamma}} H^{\frac{1}{2}}\left(\mathbb{1}-E_{0}\right)
$$

then for consistency

$$
\Gamma^{+}=\frac{\mathbb{1}-E_{0}}{\Gamma}
$$

has to be proved. According to $(\overline{\mathrm{B} .8})$ this can be achieved by verifying $\Gamma \Gamma^{+}=\Gamma^{+} \Gamma=$ $\mathbb{1}-E_{0}$.

First, one observes by means of $(\overline{B .10})$ that

$$
\left(\mathbb{1}-E_{0}\right) H^{\frac{1}{2}}\left(\mathbb{1}-\widetilde{E}_{0}\right)=\left(\mathbb{1}-E_{0}\right) H^{\frac{1}{2}} .
$$

In a second step, consider

$$
\begin{aligned}
\Gamma^{+} \Gamma & =\left(\mathbb{1}-E_{0}\right) H^{\frac{1}{2}} \frac{\mathbb{1}-\widetilde{E}_{0}}{\widetilde{\Gamma}} H^{\frac{1}{2}} \Gamma H^{\frac{1}{2}} H^{-\frac{1}{2}} \\
& =\left(\mathbb{1}-E_{0}\right) H^{\frac{1}{2}}\left(\mathbb{1}-\widetilde{E}_{0}\right) H^{-\frac{1}{2}}
\end{aligned}
$$

By employing $(\overline{\mathrm{B} .15})$ in $(\overline{\mathrm{B} .16})$ one has $\Gamma^{+} \Gamma=\mathbb{1}-E_{0}$. The equation $\Gamma \Gamma^{+}=\mathbb{1}-E_{0}$ is shown similarly and hence $(\bar{B} .14)$ is proved. 


\section{B.4 Proof of the resistance formula (5.16)}

The relation (5.15) between the resistance and the matrix elements of the associated Moore-Penrose inverse is easily inverted. Again the shorthand notation

$$
\Gamma^{+}:=\frac{\mathbb{1}-E_{0}}{\Gamma}
$$

is used for the connectivity matrix of a connected graph $\mathscr{N}$ with $n$ vertices. The relation (5.15) between the resistances $\mathscr{R}_{i, j}$ between two vertices $i, j \in \mathscr{N}$ in terms of the matrix elements of $\Gamma^{+}$reads

$$
\mathscr{R}_{i, j}=\Gamma_{i, i}^{+}+\Gamma_{j, j}^{+}-2 \Gamma_{i, j}^{+}
$$

Summing (B.18) over $j$ and $i, j$ respectively yields

$$
\sum_{j=1}^{n} \mathscr{R}_{i, j}=n \Gamma_{i, i}^{+}+\operatorname{Tr} \Gamma^{+} \quad \sum_{i, j=1}^{n} \mathscr{R}_{i, j}=2 n \operatorname{Tr} \Gamma^{+}
$$

These relations imply

$$
\begin{aligned}
\mathscr{R}_{i, j}-\frac{1}{n} \sum_{k=1}^{n} \mathscr{R}_{i, k}-\frac{1}{n} \sum_{k=1}^{n} \mathscr{R}_{k, j}+\frac{1}{n^{2}} \sum_{k, l=1}^{n} \mathscr{R}_{k, l} & =\mathscr{R}_{i, j}-\Gamma_{i, i}^{+}-\Gamma_{j, j}^{+} \\
& =-2 \Gamma_{i, j}^{+},
\end{aligned}
$$

where the first equation follows from (B.20) and and the second equation employs (B.19). This proves formula (5.16). 


\section{Appendix C}

\section{Correlation functions for the Rouse-Zimm Langevin equation}

The displacement of monomer $i$ in the Zimm model in the absence of an external flow is given by

$$
\begin{aligned}
\boldsymbol{R}_{i}(t)-\boldsymbol{R}_{i}(0)=\sum_{j=1}^{N} & {\left[H^{\frac{1}{2}} U\left(-t_{0}\right)(U(t)-\mathbb{1}) H^{-\frac{1}{2}}\right]_{i, j} \boldsymbol{R}_{j}\left(t_{0}\right) } \\
& +\int_{t_{0}}^{t} \mathrm{~d} t^{\prime}\left[H^{\frac{1}{2}} U\left(t-t^{\prime}\right) H^{-\frac{1}{2}}\right]_{i, j} \boldsymbol{\eta}_{j}\left(t^{\prime}\right) \\
& -\int_{t_{0}}^{0} \mathrm{~d} t^{\prime}\left[H^{\frac{1}{2}} U\left(-t^{\prime}\right) H^{-\frac{1}{2}}\right]_{i, j} \boldsymbol{\eta}_{j}\left(t^{\prime}\right) .
\end{aligned}
$$

with white noise $\boldsymbol{\eta}_{j}(t)$ which is characterized by Eq. (2.34). Considering $\overline{\left(\boldsymbol{R}_{i}(t)-\boldsymbol{R}_{i}(0)\right)\left(\boldsymbol{R}_{i}(t)-\boldsymbol{R}_{i}(0)\right)^{\dagger}}$ in the limit $t_{0} \rightarrow-\infty$ the term involving $\boldsymbol{R}_{i}\left(t_{0}\right) \boldsymbol{R}_{j}\left(t_{0}\right)^{\dagger}$ vanishes and so do the linear terms in $\boldsymbol{\eta}_{i}(t)$. It remains to compute

$$
\begin{aligned}
\frac{\zeta}{2 k_{\mathrm{B}} T} \lim _{t_{0} \rightarrow-\infty} \overline{\left(\boldsymbol{R}_{i}(t)-\boldsymbol{R}_{i}(0)\right)\left(\boldsymbol{R}_{j}(t)-\boldsymbol{R}_{j}(0)\right)^{\dagger}}= \\
\quad\left[H^{\frac{1}{2}}\left(\int_{t_{0}}^{t} \mathrm{~d} t^{\prime} U\left(2\left(t-t^{\prime}\right)\right)+\int_{t_{0}}^{0} \mathrm{~d} t^{\prime} U\left(-2 t^{\prime}\right)-2 \int_{t_{0}}^{0} \mathrm{~d} t^{\prime} U\left(t-2 t^{\prime}\right)\right) H^{\frac{1}{2}}\right]_{i, j} \mathbf{1} .
\end{aligned}
$$

The integrals in brackets are carried out immediately by observing

$$
\int_{t_{1}}^{t_{2}} \mathrm{~d} t^{\prime} U\left(-2 t^{\prime}\right)=\left(t_{2}-t_{1}\right) \widetilde{E}_{0}+t_{\text {mic }} \frac{\mathbb{1}-\widetilde{E}_{0}}{6 \widetilde{\Gamma}}\left(U\left(-2 t_{2}\right)-U\left(-2 t_{1}\right)\right)
$$


and $U\left(t_{1}+t_{2}\right)=U\left(t_{1}\right) U\left(t_{2}\right)$, yielding

$$
\begin{aligned}
(\ldots)= & t_{\text {mic }} U(2 t) \frac{\mathbb{1}-\widetilde{E}_{0}}{6 \widetilde{\Gamma}}\left(U(-2 t)-U\left(-2 t_{0}\right)\right)+t_{\text {mic }} \frac{\mathbb{1}-\widetilde{E}_{0}}{6 \widetilde{\Gamma}}\left(\mathbb{1}-U\left(-2 t_{0}\right)\right) \\
& -t_{\text {mic }} 2 U(t) \frac{\mathbb{1}-\widetilde{E}_{0}}{6 \widetilde{\Gamma}}\left(\mathbb{1}-U\left(-2 t_{0}\right)\right)+t \widetilde{E}_{0} \\
\stackrel{t_{0} \rightarrow-\infty}{=} t_{\text {mic }} \frac{\mathbb{1}-\widetilde{E}_{0}}{3 \widetilde{\Gamma}}(\mathbb{1}-U(t))+t \widetilde{E}_{0} & \\
= & \int_{0}^{t} \mathrm{~d} t^{\prime} U\left(t^{\prime}\right)
\end{aligned}
$$

and eventually

$$
\boldsymbol{D}_{i, j}(t)=\frac{2 k_{\mathrm{B}} T}{\zeta} \int_{0}^{t} \mathrm{~d} t^{\prime}\left[H^{\frac{1}{2}} U\left(t^{\prime}\right) H^{\frac{1}{2}}\right]_{i, j} \mathbf{1} .
$$

\section{C.1 Long time behaviour}

Note, that the co-variance matrix $\boldsymbol{D}(t)$ is positive definite. For long times it is dominated by the diffusive motion of the individual monomers. If the diffusive contribution is separated the integrand in (C.4) becomes

$$
\begin{aligned}
D(t) & =\frac{2 k_{\mathrm{B}} T}{\zeta} \int_{0}^{t} \mathrm{~d} t^{\prime} H^{\frac{1}{2}}\left(\widetilde{E}_{0}+\mathbb{1}-\widetilde{E}_{0}\right) U\left(t^{\prime}\right) H^{\frac{1}{2}} \\
& =\frac{2 k_{\mathrm{B}} T}{\zeta}\left(\frac{E_{0} t}{\operatorname{Tr}\left(E_{0} H^{-1}\right)}+\int_{0}^{t} \mathrm{~d} t^{\prime} H^{\frac{1}{2}}\left(\mathbb{1}-\widetilde{E}_{0}\right) U\left(t^{\prime}\right) H^{\frac{1}{2}}\right) \\
& \stackrel{t \rightarrow \infty}{\sim} \frac{2 k_{\mathrm{B}} T}{\zeta} \frac{E_{0} t}{\operatorname{Tr}\left(E_{0} H^{-1}\right)}+\frac{2 a^{2}}{3} H^{\frac{1}{2}} \frac{\mathbb{1}-\widetilde{E}_{0}}{\widetilde{\Gamma}} H^{\frac{1}{2}}
\end{aligned}
$$

In the second step, the representation (B.10) has been employed. 


\section{Appendix D}

\section{Equilibrium averages}

\section{D.1 Pair distributions}

By computing equilibrium averages with respect to the Boltzmann weight of the potential energy (2.15) care has to be taken due to the zero eigenvalues of the connectivity matrix. Here, a confining potential $3 \omega /\left(2 a^{2}\right) \sum_{i=1}^{N} \boldsymbol{R}_{i} \cdot \boldsymbol{R}_{i}$ is added to (2.15) and the limit $\omega \downarrow 0$ is performed subsequently, viz

$$
\langle\bullet\rangle_{\text {eq }}^{\omega}:=\frac{1}{\mathscr{Z}_{\omega}} \int_{\mathbb{R}^{3 N}} \mathrm{~d}^{3 N} R \exp \left\{-\frac{3}{2 a^{2}} \sum_{i, j=1}^{N}\left(\Gamma_{i, j}+\omega \delta_{i, j}\right) \boldsymbol{R}_{i} \cdot \boldsymbol{R}_{j}\right\}(\bullet)
$$

with a normalization $\mathscr{Z}_{\omega}$ such that $\langle 1\rangle_{\text {eq }}^{\omega}=1$. Of special interest is the probability distribution of the vector distance vector $\left(\boldsymbol{R}_{i}-\boldsymbol{R}_{j}\right)$ between two monomers $i$ and $j$

$$
p_{i, j}^{\omega}(|\boldsymbol{r}|):=\left\langle\delta\left(\left(\boldsymbol{R}_{i}-\boldsymbol{R}_{j}\right)-\boldsymbol{r}\right)\right\rangle_{\mathrm{eq}}^{\omega}
$$

which must be invariant under rotations in $\mathbb{R}^{3}$ due to the rotational invariance of the potential energy (2.15). The Fourier representation of the delta function in (D.2) leads to

$$
\begin{aligned}
p_{i, j}^{\omega}(|\boldsymbol{r}|) & =\frac{1}{(2 \pi)^{3}} \int_{\mathbb{R}^{3}} \mathrm{~d}^{3} q \mathrm{e}^{-\mathrm{i} \boldsymbol{q} \cdot \boldsymbol{r}}\left\langle\exp \left\{i \sum_{k=1}^{N} \boldsymbol{R}_{k} \cdot \boldsymbol{q}\left(\delta_{i, k}-\delta_{j, k}\right)\right\}\right\rangle_{\mathrm{eq}}^{\omega} \\
& =\frac{1}{(2 \pi)^{3}} \int_{\mathbb{R}^{3}} \mathrm{~d}^{3} q \mathrm{e}^{-i \boldsymbol{q} \cdot \boldsymbol{r}} \exp \left\{-\frac{1}{2} \boldsymbol{q}^{2} \sum_{k, l=1}^{N} \frac{a^{2}}{3} R_{k, l}(\omega)\left(\delta_{i, k}-\delta_{j, k}\right)\left(\delta_{i, l}-\delta_{j, l}\right)\right\}
\end{aligned}
$$

which involves the resolvent $R(\omega):=(\Gamma+\omega \mathbb{1})^{-1}$ of $\Gamma$. By introducing the variance

$$
\begin{aligned}
\sigma_{\omega}^{2}: & =\frac{a^{2}}{3} \sum_{k, l=1}^{N} R_{k, l}(\omega)\left(\delta_{i, k}-\delta_{j, k}\right)\left(\delta_{i, l}-\delta_{j, l}\right) \\
& =\frac{a^{2}}{3}\left(R_{i, i}(\omega)+R_{j, j}(\omega)-2 R_{i, j}(\omega)\right)
\end{aligned}
$$


and performing the remaining Fourier transform of the Gaussian in (D.3) one has

$$
p_{i, j}^{\omega}(|\boldsymbol{r}|)=\left(\frac{1}{2 \pi \sigma_{\omega}^{2}}\right)^{3 / 2} \exp \left\{-\frac{1}{2 \sigma_{\omega}^{2}} \boldsymbol{r}^{2}\right\}
$$

The limit $\omega \downarrow 0$ is meaningful only if monomers $i$ and $j$ are in the same cluster. This can be seen by the expansion of the resolvent $R(\omega)$ in (B.6) for small $\omega$ which amounts to

$$
\lim _{\omega \downarrow 0} \sigma_{\omega}^{2}= \begin{cases}\mathscr{R}_{i, j} a^{2} / 3 & \mathscr{N}(i)=\mathscr{N}(j) \\ \infty & \mathscr{N}(i) \neq \mathscr{N}(j)\end{cases}
$$

The quantity $\mathscr{R}_{i, j}$ is then defined in terms of the matrix elements of the Moore Penrose inverse of $\Gamma$

$$
\mathscr{R}_{i, j}:=\left[\frac{1-E_{0}}{\Gamma}\right]_{i, i}+\left[\frac{1-E_{0}}{\Gamma}\right]_{j, j}-2\left[\frac{1-E_{0}}{\Gamma}\right]_{i, j}
$$

\section{D.2 Preaveraging of the mobility matrix}

The equilibrium averaged hydrodynamic interaction tensor

$$
\left\langle\boldsymbol{\Omega}\left(\boldsymbol{R}_{i}-\boldsymbol{R}_{j}\right)\right\rangle_{\mathrm{eq}}=\lim _{\omega \downarrow 0} \int_{\mathbb{R}^{3}} \mathrm{~d}^{3} r p_{i, j}^{\omega}(|\boldsymbol{r}|) \boldsymbol{\Omega}(\boldsymbol{r})
$$

for $i \neq j$ is considered. Thereby, $\Omega$ denotes either the Oseen tensor $(2.8)$ which is written as

$$
\boldsymbol{\Omega}(\boldsymbol{r})=\frac{1}{8 \pi \eta_{\mathrm{s}}} \Theta(r)\left[\frac{1}{r}\left(\mathbf{1}+\hat{\boldsymbol{r}} \hat{\boldsymbol{r}}^{\dagger}\right)\right]
$$

or the Rotne-Prager tensor (2.14) which is written in the form

$$
\begin{aligned}
& \boldsymbol{\Omega}(\boldsymbol{r})=\frac{1}{8 \pi \eta_{\mathrm{s}}}\left\{\Theta\left(r-2 r_{\mathrm{m}}\right)\left[\frac{1}{r}\left(\mathbf{1}+\hat{\boldsymbol{r}} \hat{\boldsymbol{r}}^{\dagger}\right)+\frac{2 r_{\mathrm{m}}^{2}}{3 r^{3}}\left(\mathbf{1}-3 \hat{\boldsymbol{r}} \hat{\boldsymbol{r}}^{\dagger}\right)\right]\right. \\
&\left.+\Theta\left(2 r_{\mathrm{m}}-r\right)\left[\frac{4}{3 r_{\mathrm{m}}} \mathbf{1}+\frac{3 r}{8 r_{\mathrm{m}}^{2}}\left(\mathbf{1}-\frac{1}{3} \hat{\boldsymbol{r}} \hat{\boldsymbol{r}}^{\dagger}\right)\right]\right\}
\end{aligned} .
$$

Here, the Heaviside step function $\Theta(x)$ equals 1 for $x \geq 0$ and zero otherwise. By introducing spherical coordinates in (D.8) the integral factorizes into a radial and an angular average according to

$$
\begin{gathered}
\langle\bullet\rangle_{\text {rad }}^{\omega}:=\int_{0}^{\infty} \mathrm{d} r 4 \pi r^{2} p_{i, j}^{\omega}(r)(\bullet) \\
\langle\bullet\rangle_{\text {ang }}:=\frac{1}{4 \pi} \int_{0}^{2 \pi} \mathrm{d} \varphi \int_{0}^{\pi} \mathrm{d} \vartheta \sin (\vartheta)(\bullet)
\end{gathered}
$$


The average over $\vartheta, \varphi$ is carried out first yielding $\left\langle\hat{\boldsymbol{r}} \hat{\boldsymbol{r}}^{\dagger}\right\rangle_{\text {ang }}=1 / 3$. For the radial average it is convenient to introduce the quantities

$$
I_{[s, t]}^{(m)}(\omega):=\left\langle r^{m} \chi_{[s, t]}(r)\right\rangle_{\mathrm{rad}}^{\omega}
$$

with $\chi_{[s, t]}(r)$ denoting the characteristic function on the interval $[s, t] \subset[0, \infty)$. Obviously, if $s=0$ in (D.13) convergence requires $m>-3$. A simple substitution gives

$$
I_{[s, t]}^{(m)}(\omega)=\frac{2}{\sqrt{\pi}}\left(2 \sigma_{\omega}^{2}\right)^{\frac{m}{2}}\left[\gamma\left(\frac{m+3}{2}, \frac{s^{2}}{2 \sigma_{\omega}^{2}}\right)-\gamma\left(\frac{m+3}{2}, \frac{t^{2}}{2 \sigma_{\omega}^{2}}\right)\right]
$$

in terms of the incomplete Gamma function $\gamma(\alpha, x):=\int_{0}^{x} \mathrm{~d} r \exp (-r) r^{\alpha-1}$.

In terms of $(\overline{\mathrm{D} .13})$, the average $(\overline{\mathrm{D} .8})$ is written as

$$
\left\langle\boldsymbol{\Omega}\left(\boldsymbol{R}_{i}-\boldsymbol{R}_{j}\right)\right\rangle_{\mathrm{eq}}=\frac{1}{6 \pi \eta_{\mathrm{s}}} \mathbf{1} \begin{cases}I_{[0, \infty)}^{(-1)}(0), & \text { Oseen } \\ I_{\left[2 r_{\mathrm{m}}, \infty\right)}^{(-1)}(0)+\frac{1}{r_{\mathrm{m}}} I_{\left[0,2 r_{\mathrm{m}}\right]}^{(0)}(0)-\frac{1}{4 r_{\mathrm{m}}^{2}} I_{\left[0,2 r_{\mathrm{m}}\right]}^{(1)}(0), & \text { Rotne-Prager }\end{cases}
$$

The limit $\omega \downarrow 0$ of (D.14) depends on whether $i$ and $j$ belong to the same cluster or not.

1) $\mathscr{N}(i) \neq \mathscr{N}(j)$ : The variance $\sigma_{\omega}$ tends to infinity for $\omega \downarrow 0$ according to (D.6). The asymptotic expansion $\gamma(\alpha, x)=\alpha^{-1} x^{\alpha}+\mathscr{O}\left(x^{\alpha+1}\right)$ of the incomplete $\gamma$-function for $x \downarrow 0$ [Ch. 9.2, Eq. (4) in [ErMa53] Vol. 2] implies $I_{[s, t]}^{(m)}(\omega)=\operatorname{const} \sigma_{\omega}^{-3}+\mathscr{O}\left(\sigma_{\omega}^{-4}\right)$ for $\sigma_{\omega} \rightarrow \infty$ and finite interval limits $s, t$ and eventually $\lim _{\omega \downarrow 0} I_{[s, t]}^{(m)}(\omega)=0$. For $t=\infty$ one has $\lim _{\omega \downarrow 0} I_{[s, \infty)}^{(m)}(\omega)=0$ iff $m<0$ which is sufficient for all cases in (D.15). In summary, $H_{i, j}=0$ whenever $i$ and $j$ belong to different clusters.

2) $\mathscr{N}(i)=\mathscr{N}(j)$ : The variance $\sigma_{\omega}^{2}$ tends to the finite value $\sigma_{0}^{2}=\mathscr{R}_{i, j} a^{2} / 3$ for $\omega \rightarrow 0$. In case of the Oseen-tensor this implies

$$
\left\langle\boldsymbol{\Omega}\left(\boldsymbol{R}_{i}-\boldsymbol{R}_{j}\right)\right\rangle_{\mathrm{eq}}=\frac{1}{6 \pi \eta_{\mathrm{s}}} \frac{2}{\sqrt{\pi}}\left(\frac{1}{2 \sigma_{0}^{2}}\right)^{1 / 2} \gamma(1, \infty) \mathbf{1}
$$

with $\gamma(1, \infty)=\Gamma(1)=1$. For the Rotne-Prager tensor one has

$$
\begin{aligned}
\left\langle\boldsymbol{\Omega}\left(\boldsymbol{R}_{i}-\boldsymbol{R}_{j}\right)\right\rangle_{\mathrm{eq}}=\frac{1}{6 \pi \eta_{\mathrm{s}}} \frac{2}{\sqrt{\pi}} & \left\{\left(\frac{1}{2 \sigma_{0}^{2}}\right)^{1 / 2}\left[\gamma(1, \infty)-\gamma\left(1, \frac{2 r_{\mathrm{m}}^{2}}{\sigma_{0}^{2}}\right)\right]\right. \\
& \left.+\frac{1}{r_{\mathrm{m}}} \gamma\left(\frac{3}{2}, \frac{2 r_{\mathrm{m}}^{2}}{\sigma_{0}^{2}}\right)-\frac{\left(2 \sigma_{0}^{2}\right)^{1 / 2}}{4 r_{\mathrm{m}}^{2}} \gamma\left(2, \frac{2 r_{\mathrm{m}}^{2}}{\sigma_{0}^{2}}\right)\right\} \mathbf{1}
\end{aligned}
$$

The incomplete Gamma-functions for $m=-1,1,0$ are then expressed in a more convenient way: According to eq. 8.352 in [GrRh80] one has $\gamma(1, x)=(1-$ $\exp (-x)$ and $\gamma(2, x)=(1-\exp (-x)(1+x))$. Equations 8.356.1 and 8.359.4 give $\gamma(3 / 2, x)=\sqrt{\pi} / 2 \operatorname{erf}(\sqrt{x})-\sqrt{x} \exp (-x)$ in terms of the error function $\operatorname{erf}(x):=$ 
$2 / \sqrt{\pi} \int_{0}^{x} \mathrm{~d} r \exp \left(-r^{2}\right)$. In summary, (D.17) is then expressed in the variable $x=2 r_{\mathrm{m}}^{2} / \sigma_{0}^{2}$ via

$$
\begin{aligned}
\left\langle\boldsymbol{\Omega}\left(\boldsymbol{R}_{i}-\boldsymbol{R}_{j}\right)\right\rangle_{\mathrm{eq}}=\frac{1}{6 \pi \eta_{\mathrm{s}}} \frac{2}{\sqrt{\pi}} & \left\{\frac{1}{2 r_{\mathrm{m}}} \sqrt{x} \mathrm{e}^{-x}+\frac{1}{r_{\mathrm{m}}}\left(\frac{\sqrt{\pi}}{2} \operatorname{erf}(\sqrt{x})-\sqrt{x} \mathrm{e}^{-x}\right)\right. \\
& \left.-\frac{1}{2 r_{\mathrm{m}}} \frac{1}{\sqrt{x}}\left(1-(1+x) \mathrm{e}^{-x}\right)\right\} \mathbf{1}
\end{aligned}
$$

By introducing the parameter

$$
\kappa:=\sqrt{\frac{6}{\pi}} \frac{\zeta}{6 \pi \eta_{\mathrm{s}} a}=\sqrt{\frac{6}{\pi}} \frac{r_{\mathrm{m}}}{a}
$$

one has $x=2 r_{\mathrm{m}}^{2} / \sigma_{0}^{2}=6 r_{\mathrm{m}}^{2} /\left(\mathscr{R}_{i, j} a^{2}\right)=\pi \kappa^{2} / \mathscr{R}_{i, j}$. As a result the preaveraged mobility matrix (2.10) is block-diagonal with respect to the clusters and within one block given by

$$
\begin{aligned}
\left\langle\boldsymbol{H}_{i, j}\left(\boldsymbol{R}_{i}-\boldsymbol{R}_{j}\right)\right\rangle_{\mathrm{eq}} & =\delta_{i, j} \mathbf{1}-\left(1-\delta_{i, j}\right) \zeta\left\langle\boldsymbol{\Omega}\left(\boldsymbol{R}_{i}-\boldsymbol{R}_{j}\right)\right\rangle_{\mathrm{eq}} \\
& =\left[\delta_{i, j}+\left(1-\delta_{i, j}\right) h\left(\frac{\kappa^{2} \pi}{\mathscr{R}_{i, j}}\right)\right] \mathbf{1} .
\end{aligned}
$$

with the function $h$ depends on the hydrodynamic interaction tensor

$$
h(x)= \begin{cases}\sqrt{x / \pi} & \text { Oseen } \\ \operatorname{erf}(\sqrt{x})-\frac{1}{\sqrt{\pi}} \frac{1-\exp (-x)}{\sqrt{x}} & \text { Rotne-Prager }\end{cases}
$$

The latter reduces to the first as $x \downarrow 0$. 


\section{Appendix E}

\section{Asymptotic evaluations of integrals and sums}

\section{E.1 Critical behaviour of disorder averages}

Here, the evaluation of disorder averages (5.11), or more generally, moments of the cluster size distribution are considered. If $(5.11)$ is rewritten according to

$$
\sum_{n=1}^{\infty} n^{j-\tau+b} f\left(n \varepsilon^{1 / \sigma}\right)=\varepsilon^{-(j+1-\tau+b) \sigma}\left\{\varepsilon^{1 / \sigma} \sum_{n=1}^{\infty}\left(n \varepsilon^{1 / \sigma}\right)^{j-\tau+b} f\left(n \varepsilon^{1 / \sigma}\right)\right\} .
$$

for $\varepsilon \downarrow 0$ the term in the curly braces is identified with the Riemann sum of the dimensionless integral $\int_{n=0}^{\infty} \mathrm{d} x x^{j-\tau+b} f(x)$. Since the scaling function $f$ approaches a constant for $x \downarrow 0$ and decays exponentially for $x>1$ the integral is well defined if the singularity at the origin is integrable, viz.

$$
j-\tau+b>-1 \Leftrightarrow j+1-\tau+b>0
$$

In this case the critical behaviour

$$
\sum_{n=1}^{\infty} n^{j} \tau_{n} n^{b} \sim \varepsilon^{-(j+1-\tau+b) / \sigma}
$$

is deduced. It requires a divergence for $\varepsilon \downarrow 0$, i.e. $j+1-\tau+b>0$. If instead $j+1-\tau+b<0$ the divergence of the integral due to the singularity at the origin and the prefactor in $(\bar{E} .1)$ cancel out and the sum converges to a finite value for $\varepsilon \downarrow 0$. In this case (E.1) exhibits no divergence at the critical point. For the most important case $j=1$, the critical behaviour of the disorder average $(\mathrm{E} .1)$ is given by

$$
\sum_{n=1}^{\infty} n \tau_{n} n^{b} \sim \varepsilon^{-(2-\tau+b) / \sigma}
$$


A second application is the weight averaged cluster size which follows from $j=1$ and $b=1$

$$
n_{\mathrm{w}}=\sum_{n=1}^{\infty} n^{2} \tau_{n} \sim \varepsilon^{-(3-\tau) / \sigma}
$$

\section{E.2 Long-time decay of the incoherent scattering function}

Consider the long time limit of the incoherent scattering function

$$
S(\boldsymbol{q}, t) \sim \sum_{n=1}^{\infty} n \tau_{n} \mathrm{e}^{-D_{n} q^{2} t}
$$

with the diffusion constant $D_{n} \sim n^{-b}$. Hence, the Rouse model is recovered for $b=1$ and the Zimm model for $b=d_{\mathrm{f}}^{(\mathrm{G})}$. The exponent in (E.6) is rewritten by observing $D_{n} q^{2} t=$ $\left(t / t_{q}\right)\left(n^{*} / n\right)^{b}$ with $t_{q}:=t_{\text {mic }} q^{-2} \varepsilon^{-\tilde{z}}$ with $\tilde{z}=b / \sigma$. The scaling function of the cluster size distribution $\tau_{n} \sim n^{-\tau} f\left(n / n^{*}\right)$ is replaced by an exponential which is asymptotically valid for large $n / n^{*}$ and the sum is converted into an integral

$$
\begin{aligned}
S(\boldsymbol{q}, t) & \sim \int_{1}^{\infty} \mathrm{d} n n^{1-\tau} \mathrm{e}^{-n / n^{*}} \mathrm{e}^{-\left(t / t_{q}\right)\left(n / n^{*}\right)^{-b}} \\
& =\varepsilon^{(\tau-2) / \sigma} \int_{0}^{\infty} \mathrm{d} x x^{1-\tau} \mathrm{e}^{-x} \mathrm{e}^{-\left(t / t_{q}\right) x^{-b}} .
\end{aligned}
$$

In the second step $x=n / n^{*}$ is substituted and the lower limit of integration is extended to $x=0$, since the second exponential decays rapidly for $x \downarrow 0$. Now, an exponent $\delta$ is defined in order to rewrite the $\varepsilon$ dependent prefactor according to $\varepsilon^{(\tau-2) / \sigma}=\left(q^{2} t\right)^{-\delta}\left(t / t_{q}\right)^{\delta}$. This requires $\delta=(\tau-2) /(\tilde{z} \sigma)=(\tau-2) / b$ and $(\bar{E} .7)$ can be written in the scaling form

$$
S(\boldsymbol{q}, t) \sim\left(q^{2} t\right)^{-\delta} \mathcal{F}_{S}\left(t / t_{q}\right),
$$

where the scaling function is defined by

$$
\mathcal{F}_{S}(y)=y^{\delta} \int_{0}^{\infty} \mathrm{d} x x^{1-\tau} \exp \left\{-\left(x+y x^{-b}\right\}\right.
$$

First, the limit $y \ll 1$ of $(\overline{\mathrm{E} .10})$ is considered. A substitution $x=w y^{1 / b}$ yields

$$
\mathcal{F}_{S}(y)=y^{\delta} y^{(2-\tau) / b} \int_{0}^{\infty} \mathrm{d} w w^{1-\tau} \exp \left\{-\left(w y^{1 / b}+w^{-b}\right)\right\}
$$

where the integral tends to to a finite limit for $y \downarrow 0$.

Second, for $y \gg 1$ (E.10) can be evaluated by Laplace's method (see [BeOr78] Ch. 6). Therefore, $x=w y^{1 /(1+b)}$ is substituted which gives

$$
\mathcal{F}_{S}(y)=y^{\delta} y^{(2-\tau) /(1+b)} \int_{0}^{\infty} \mathrm{d} w w^{1-\tau} \exp \left\{-y^{1 /(1+b)}\left(w+w^{-b}\right)\right\}
$$


The function $g(w)=w+w^{-b}$ has a unique minimum at $w_{0}=b^{1 /(1+b)}$. By taking into account the algebraic prefactor which stems from the expansion of $g(w)$ around the minimum (see. Ch. 6.4 [BeOr78] for details) the scaling function takes the form

$$
\mathcal{F}_{S}(y) \sim y^{\delta} y^{(2-\tau) /(1+b)} y^{-1 /(2(1+b))} \mathrm{e}^{-\lambda y^{1 /(1+b)}}
$$

for $y \rightarrow \infty$. Here $\lambda$ is a constant of order unity. By denoting $\alpha_{S}=1 /(1+b)$ as the exponent of the stretched exponential the two limiting cases of the scaling function are given summarized as

$$
\mathcal{F}_{S}(y)= \begin{cases}\mathscr{O}(1), & y \ll 1 \\ y^{\alpha(\delta-1 / 2)} \exp \left\{-\lambda y^{\alpha}\right\}, & y \gg 1\end{cases}
$$

\section{E.3 Moments of the resistance distribution for random trees}

The $p$-th moment of the resistance (distance) distribution in labelled random trees is given by

$$
\begin{aligned}
\left\langle\mathscr{R}_{i, j}^{p}\right\rangle_{n} & =(n-2) ! \sum_{k=1}^{n-1} \frac{k^{p}(k+1)}{n^{k}(n-k-1) !} \\
& =\frac{1}{n-1} \sum_{k=1}^{n-1} k^{p}(k+1) \frac{(n-1)(n-2) \ldots(n-k)}{n^{k}}
\end{aligned}
$$

By converting the fraction in the sum in $(\mathrm{E} .15)$ into

$$
\begin{aligned}
\prod_{l=1}^{k}(1-l / n) & =\exp \left\{\sum_{l=1}^{k} \ln (1-l / n)\right\} \\
& =\exp \left\{n \int_{0}^{k / n} \mathrm{~d} y \ln (1-y)\right\}
\end{aligned}
$$

and defining the function $f(x):=\int_{0}^{x} \mathrm{~d} y \ln (1-y)$ the average resistance can be written as

$$
\begin{aligned}
\left\langle\mathscr{R}_{i, j}^{p}\right\rangle_{n} & =n^{p+1} \frac{1}{n-1} \sum_{k=1}^{n-1}\left(\frac{k}{n}\right)^{p+1} \exp \{n f(k / n)\} \\
& \sim n^{p+1} \int_{0}^{1} \mathrm{~d} x x^{p+1} \exp \{n f(x)\}
\end{aligned}
$$

For the second equation in (E.17), the (Riemann) sum has been replaced by an integral which is valid for large $n$. This integral is now evaluated asymptotically for $n \rightarrow \infty$ by 
Laplace's method. Therefore, one observes that the function $f(x)$ has a unique maximum at $x=0$ in $[0,1]$. According to [BeOr78], Ch. 6.4 the asymptotic behaviour of the integral for large $n$ remains the same, if $f$ is replaced by the expansion $f(x)=-x^{2} / 2+\mathscr{O}\left(x^{3}\right)$ around its maximum point. Hence, for $n \rightarrow \infty$

$$
\left\langle\mathscr{R}_{i, j}^{p}\right\rangle_{n} \sim n^{p+1} \int_{0}^{1} \mathrm{~d} x x^{p+1} \exp \left\{-\frac{n}{2} x^{2}\right\}
$$

By substituting $x=n x^{2} / 2$ the integral takes the form of an incomplete Gamma function

$$
n^{-(1+p / 2)} 2^{p / 2} \int_{0}^{n / 2} \mathrm{~d} y y^{p / 2} \exp \{-y\} \sim n^{-(1+p / 2)} 2^{p / 2} \Gamma(1+p / 2)
$$

which is tends to the complete Gamma function for $n \rightarrow \infty$. Eventually, this yields the asymptotic behaviour

$$
\left\langle\mathscr{R}_{i, j}^{p}\right\rangle_{n} \sim 2^{p / 2} \Gamma(1+p / 2) n^{p / 2}
$$

of the $p$-th moment of the resistance distribution for labelled trees of size $n$. The result (E.20) for $p=1,2$ agrees with the rigorous result computed in [MeMo70]. 


\section{Appendix F}

\section{The Jensen inequality}

Probably one of the most useful inequalities for problems involving randomness is the Jensen inequality. Consider the problem of computing the average of a function $g(X)$ where $X$ is a random variable with given probability distribution $p(X)$. Depending on the function $g$, this turns out to be a highly unfeasible task in general. In contrast, it is often relatively simple to compute the average of the random variable $X$. Under certain conditions, the Jensen inequality allows to relate the average value of $g$ to the value which $g$ assumes at the average of $X$ (see e.g. [Fel66] Vol. II, Ch. V.8)

More precisely, let $X \in \mathbb{R}$ be a random variable which is characterized by its probability distribution $p(X)$ which is concentrated on an interval $I \subset \mathbb{R}$ and suppose that the expectation value $\bar{X}:=\int_{I} \mathrm{~d} X X p(X)$ exists. Let $g: I \rightarrow \mathbb{R}$ be a convex, real valued function defined on $I \subset \mathbb{R}$. Whenever the expectation $\bar{g}:=\int_{I} \mathrm{~d} X g(X) p(X)$ exists the Jensen inequality states

$$
\bar{g} \geq g(\bar{X}) .
$$

The convexity condition for a twice differentiable function $g$ is indeed equivalent to a nonnegative, second order derivative of $g$ for all $X \in I$.

A generalization of the Jensen inequality is the Jensen-Peierls inequality for operators. Here it is given for real, finite dimensional, symmetric matrices. In fact for this special case it is equivalent to (F.1) [Sim79].

Let $H$ be a real, symmetric matrix of order $n, E$ a one dimensional, orthogonal projector, i.e. $E^{2}=E$, and $g: I \rightarrow \mathbb{R}$ a convex, real-valued function, defined on an interval $I \subset \mathbb{R}$. Suppose, that the spectrum $\sigma(H)$ of $H$, that is the set of real eigenvalues, is contained in $I$. Then the Jensen-Peierls inequality states

$$
\operatorname{Tr}(E g(H)) \geq g(\operatorname{Tr}(E H))
$$

For a proof and generalizations see [Sim79] Sec. 8c.

By specifying $E$ as the projector $E_{0}=E_{0}\left(\mathscr{N}_{k}\right)$ onto the nullspace of $\Gamma\left(\mathscr{N}_{k}\right), H$ as the preaveraged mobility matrix and $g(x)=x^{-1}$ as the convex function the inequality yields

$$
\operatorname{Tr}\left(E_{0} H^{-1}\right) \geq\left(\operatorname{Tr}\left(E_{0} H\right)\right)^{-1}
$$


which gives the inequality (4.49) between the diffusion constant of a cluster and the Kirkwood diffusion constant. 


\section{Appendix G}

\section{Disorder averages from the Leath-algorithm}

Here, it is demonstrated how the disorder average $A=\langle A(\cdot)\rangle$ of an observable of type (5.2) is related to a sequence of clusters $\left\{\mathscr{L}_{l}\right\}_{l=1}^{L}$ which is generated by the Leath algorithm.

Therefore, consider the average number of lattice points per vertex which belong to a $\mathscr{N}$-cluster

$$
\tau(\mathscr{N}):=\left\langle\frac{1}{N} \sum_{k=1}^{K} N_{k} \delta_{\mathscr{N}_{k}, \mathscr{N}}\right\rangle
$$

which amounts to express $A=\langle A(\cdot)\rangle$ via

$$
A=\left\langle\sum_{k=1}^{K} \frac{N_{k}}{N} f\left(\mathscr{N}_{k}\right)\right\rangle=\sum_{\mathscr{N}} \tau(\mathscr{N}) f(\mathscr{N})
$$

Eq. (G.1) implies

$$
\begin{aligned}
\tau(\mathscr{N}) & =\left\langle\frac{1}{N} \sum_{k=1}^{K} N_{k} \delta_{\mathscr{N}_{k}, \mathscr{N}}\right\rangle=\frac{1}{N} \sum_{i=0}^{N-1}\left\langle\chi_{i \in \mathscr{N}}\right\rangle \\
& =\left\langle\chi_{0 \in \mathscr{N}}\right\rangle
\end{aligned}
$$

where the last step in (G.3) is valid due to translational invariance. Hence, the quantity $\tau(\mathscr{N})$ can be interpreted as the probability that the origin belongs to a $\mathscr{N}$-cluster which provides a link to the Leath algorithm. Starting from the origin as seed vertex, the Leath algorithm generates a sequence $\left\{\mathscr{L}_{l}\right\}_{l=1}^{L}$ where the computational probability of finding the cluster $\mathscr{N}$ is given by

$$
p^{(\text {Leath })}(\mathscr{N}):=\lim _{L \rightarrow \infty} \frac{1}{L} \sum_{l=1}^{L} \delta_{\mathscr{L}_{l}, \mathscr{N}}
$$


The Leath probability (G.5) of finding the cluster $\mathscr{N}$ is the probability that the origin belongs to a $\mathscr{N}$-cluster, hence

$$
\left\langle\chi_{0 \in \mathscr{N}}\right\rangle=p^{(\text {Leath })}(\mathscr{N})
$$

By successively inserting $(\overline{\mathrm{G} .5})$ into $(\mathrm{G.6})$ into $(\mathrm{G.3})$ into $(\mathrm{G} .2)$ one ends up with

$$
A=\lim _{L \rightarrow \infty} \frac{1}{L} \sum_{l=1}^{L} \delta_{\mathscr{L}_{l}, \mathscr{N}} f\left(\mathscr{L}_{l}\right)
$$

By choosing the observable $f\left(\mathscr{N}_{k}\right):=\delta_{N_{k}, n} / N_{k}$ one has

$$
\begin{aligned}
\tau_{n} & =\left\langle\frac{1}{N} \sum_{k=1}^{K} \delta_{N_{k}, n}\right\rangle=\lim _{L \rightarrow \infty} \frac{1}{L} \sum_{l=1}^{L} \delta_{N\left(\mathscr{L}_{l}\right), n} / N\left(\mathscr{L}_{l}\right) \\
& =: \frac{1}{n} \tau_{n}^{\text {Leath }}
\end{aligned}
$$

with

$$
\tau_{n}^{\text {Leath }}:=\lim _{L \rightarrow \infty} \frac{1}{L} \sum_{l=1}^{L} \delta_{N\left(\mathscr{L}_{l}\right), n}
$$




\section{Appendix H}

\section{Ring polymers in the Zimm model}

In the following, the preaveraged Rotne-Prager tensor for ring polymers in the Zimm model is examined. Thereby, the attention is drawn to the limiting cases of small and large $\kappa$.

The eigenvalues of the commuting cyclic matrices $\Gamma\left(\mathscr{C}_{n}\right)$ and $H\left(\mathscr{C}_{n}\right)$ can be written in terms of Fourier representations. The eigenvalues of $\Gamma\left(\mathscr{C}_{n}\right)$ are given by

$$
\gamma_{l}=2(1-\cos (2 \pi l / n))
$$

for $l=0,1 \ldots n-1$. The eigenvalues of $H\left(\mathscr{C}_{n}\right)$ are given by

$$
h_{l}=\sum_{k=0}^{n-1} h\left(\kappa^{2} \pi / \mathscr{R}_{k}\right) \exp \left\{\text { i } 2 \pi \frac{k l}{n}\right\}
$$

where $\mathscr{R}_{k}=\left(k^{-1}+(n-k)^{-1}\right)^{-1}=k(1-k / n)$ is the resistance between two monomers on the ring with chemical distance $k$. Due to cyclicity $\mathscr{R}_{k}=\mathscr{R}_{n-k}$ and $(\overline{\mathrm{H}} .2)$ can be written as

$$
h_{l}=h(0)+\delta_{n, \text { even }}(-1)^{l+1} h\left(\kappa^{2} \pi / \mathscr{R}_{n / 2}\right)+2 \sum_{k=1}^{\lfloor n / 2\rfloor} h\left(\kappa^{2} \pi / \mathscr{R}_{k}\right) \cos (2 \pi l k / n)
$$

where $\lfloor x\rfloor$ denotes the floor function which returns the maximum integer $k_{x}$ which satisfies $k_{x} \leq x$. The eigenvalues of $\widehat{\Gamma}\left(\mathscr{C}_{n}\right)=\Gamma\left(\mathscr{C}_{n}\right) H\left(\mathscr{C}_{n}\right)$ are then given by $\widehat{\gamma}_{l}=\gamma_{l} h_{l}$. The function $h(x)$ can be either chosen according to the Rotne-Prager tensor $(2.31)$ or according to the Oseen tensor (2.30). In the following the inverse moments of $\widehat{\Gamma}\left(\mathscr{C}_{n}\right)$ are computed for two limiting cases.

Case 1: $n \gg 1$ and $\kappa^{2} / n \gg 1$ : Then, also $\kappa^{2} / \mathscr{R}_{k} \gg 1$ for all $k=1,2 \ldots n$ and one can expand $h(x)=\operatorname{erf}\left(x^{1 / 2}\right)-\left(1-\exp (-x) /(\pi x)^{1 / 2}\right)=1-(\pi x)^{-1 / 2}+\mathscr{O}(\exp (-x))$ for 
large arguments. This implies

$$
\begin{aligned}
h_{l}=1 & +\delta_{n, \text { even }}(-1)^{l+1}\left(1-\frac{n^{1 / 2}}{2 \kappa \pi}\right)+2 \sum_{k=1}^{\lfloor n / 2\rfloor} \cos (2 \pi l k / n) \\
& -\frac{2}{\pi} \sum_{k=1}^{\lfloor n / 2\rfloor} \frac{n^{1 / 2}}{\kappa}\left[\frac{k}{n}\left(1-\frac{k}{n}\right)\right]^{1 / 2} \cos (2 \pi l k / n)
\end{aligned}
$$

The last (Riemann) sum can be converted into an integral for $l \ll n$, it yields

$$
\begin{gathered}
\frac{2 n^{3 / 2}}{\pi \kappa} \int_{0}^{1 / 2} \mathrm{~d} x[x(1-x)]^{1 / 2} \cos (2 \pi l x)=\frac{n^{3 / 2}}{\pi \kappa} \int_{0}^{1} \mathrm{~d} x[x(1-x)]^{1 / 2} \cos (2 \pi l x) \\
=\frac{n^{3 / 2}}{\pi \kappa} \frac{\pi^{1 / 2}}{2 \pi l} \cos (\pi l) \Gamma(3 / 2) J_{1}(\pi l)=-\frac{n^{3 / 2}}{\kappa \pi} \frac{(-1)^{l+1}}{8 l} J_{1}(\pi l)
\end{gathered}
$$

The second equation follows from Eq. 3.768.9 in [GrRh80] in terms of Euler's $\Gamma$ function and the Bessel function $J_{1}$. The Bessel function can be expanded for large arguments, $J_{1}(z)=(2 /(\pi z))^{1 / 2} \cos (z-3 / 4 \pi)+\mathscr{O}\left(z^{-5 / 2}\right)$ according to Eq. 7.13.1 (3) in [ErMa53], Vol. II. If $(\overline{\mathrm{H} .5})$ is inserted into $(\overline{\mathrm{H} .4})$ by observing $\cos (\pi(l-3 / 4))=(-1)^{l+1} / \sqrt{2}$ the eigenvalues of $H$ become

$$
h_{l} \sim \frac{(n / l)^{3 / 2}}{\kappa}, \quad \text { for } \quad 1 \ll l \ll n \ll \kappa
$$

If the inverse moments of $\widehat{\Gamma}$ are computed by employing expression (․․․), one has

$$
\frac{1}{n} \sum_{l=1}^{n-1} \frac{1}{\left(\gamma_{l} h_{l}\right)^{m}} \sim \kappa^{l} \int_{1 / n}^{1} \mathrm{~d} x\left[\frac{x^{3 / 2}}{1-\cos (2 \pi x)}\right]^{m}
$$

The dominating contribution to the integral in ( $\mathrm{H} .7)$ stems form the behaviour for $x \downarrow 0$ where the integrand behaves as $x^{-m / 2}$. Hence, for large $\kappa$ or more precisely for $n \gg 1$ and $\kappa^{2} / n \gg 1$ the inverse moments take the form

$$
\frac{1}{n} \sum_{l=1}^{n-1} \frac{1}{\left(\gamma_{l} h_{l}\right)^{m}} \sim \kappa^{m} \begin{cases}\text { const }, & m=1 \\ \ln (n), & m=2 \\ n^{m / 2-1}, & m \geq 3\end{cases}
$$

Case 2: $n \gg 1$ and $\kappa^{2} / n \gg 1$ : For $\kappa^{2} / n \gg 1$ the function $h(x)$ for the Rotne-Prager tensor can be approximated by its expansion for low $x$ which is given by $h(x)=\sqrt{x / \pi}$ 
for Oseen tensor. Inserting this $(\mathrm{H} .3)$ yields

$$
\begin{aligned}
h_{l} & =1+2 n^{1 / 2} \kappa \sum_{k=1}^{\lfloor n / 2\rfloor} \frac{1}{n}\left[\frac{k}{n}\left(1-\frac{k}{n}\right)\right]^{-1 / 2} \cos (2 \pi l k / n)+\mathscr{O}\left(n^{-1}\right) \\
& \sim 1+n^{1 / 2} \kappa \int_{0}^{1} \mathrm{~d} x \frac{\cos (2 \pi l x)}{[x(1-x)]^{1 / 2}}+\mathscr{O}\left(n^{-1}\right) \\
& \sim 1+n^{1 / 2} \kappa(-1)^{l} \pi^{1 / 2} J_{0}(\pi l)+\mathscr{O}\left(n^{-1}\right)
\end{aligned}
$$

where the last equation follows from from Eq. 3.768.9 in [GrRh80]. The Bessel function can be expanded for large arguments $J_{0}(z)=(2 /(\pi z))^{1 / 2} \cos (z-\pi / 4)+\mathscr{O}\left(z^{-5 / 2}\right)$ (see, e.g. , Eq. 7.13.1 (3) in [ErMa53], Vol. II). With $\cos (\pi(l-1 / 4))=(-1)^{l} / \sqrt{2}$ this gives rise to

$$
h_{l} \sim 1+\kappa\left(\frac{n}{\pi l}\right)^{1 / 2}
$$

for $l \gg 1$ to leading order in $n$.

The $m$ th inverse moment of the $\widehat{\Gamma}$ can be computed from the approximation $(\overline{H .11})$ via

$$
\begin{aligned}
\frac{1}{n} \sum_{l=1}^{n-1} \frac{1}{\left(\gamma_{l} h_{l}\right)^{m}} & \sim \frac{1}{2} \int_{1 / n}^{1} \mathrm{~d} x[(1-\cos (2 \pi x))(1+\kappa /(\pi x))]^{-m} \\
& \sim \frac{1}{2} \int_{1 / n}^{1} \mathrm{~d} x\left[\frac{(\pi x)^{-3 / 2}}{2 \kappa}\right]^{m}
\end{aligned}
$$

where the last equation is obtained by expanding the integrand for $x \downarrow 0$ within the assumption $\kappa^{2} / \pi \gg 1 / n$. Hence, for $n \gg 1$ and $\kappa^{2} n \gg \pi$ the inverse moments take the form

$$
\frac{1}{n} \sum_{l=1}^{n-1} \frac{1}{\left(\gamma_{l} h_{l}\right)^{m}} \sim \frac{1}{3 m-2}\left(\frac{1}{2 \kappa \pi^{3 / 2}}\right)^{m} n^{\frac{3 m-2}{2}}
$$

for $m \geq 1$. If instead $\kappa^{2} / \pi \lesssim 1 / n$ in ( $\overline{\text { H.11) }}$ and (H.12), Rouse behaviour is observed. Therefore the crossover clusters size $\widehat{n}:=\pi / \kappa^{2}$ separates Rouse from Zimm behaviour. 


\section{Appendix I}

\section{A variational bound to the viscosity}

In the following it is demonstrated how it is possible to go beyond the approximation of phantom clusters and address the effects of excluded volume interactions by means of variational bounds. The advantage of this approach is that the bound to the shear viscosity can be expressed in terms of an equilibrium expectation value (see [Pra71] or Ch. 4.6 in [DoEd88]).

The starting point is the a priori unknown steady state solution $p(\{\boldsymbol{R}\})$ of the Smoluchowski equation (2.13). For vanishing shear rate $\dot{\gamma}=0$ the solution is given by the equilibrium Boltzmann weight $p_{\text {eq }}(\{\boldsymbol{R}\}) \sim \exp \left\{-U(\{\boldsymbol{R}\}) / k_{\mathrm{B}} T\right\}$. In the presence of shear flow a product ansatz

$$
p(\{\boldsymbol{R}\})=: p_{\text {eq }}(\{\boldsymbol{R}\}) p_{\Delta}(\{\boldsymbol{R}\})
$$

is made, with an unknown function $p_{\Delta}(\{\boldsymbol{R}\})$ which is required to be translationally invariant

$$
p_{\Delta}\left(\boldsymbol{R}_{1}, \boldsymbol{R}_{2}, \ldots \boldsymbol{R}_{N}\right)=p_{\Delta}\left(\boldsymbol{R}_{1}+\boldsymbol{a}, \boldsymbol{R}_{2}+\boldsymbol{a}, \ldots \boldsymbol{R}_{N}+\boldsymbol{a}\right) .
$$

As shown by Prager [Pra71], for each $p_{\Delta}$ satisfying (I.2) a lower bound for the shear viscosity is given by

$$
\eta \geq \frac{k_{\mathrm{B}} T}{\dot{\gamma}^{2} N} W\left[p_{\Delta}\right]
$$

with a functional

$$
\begin{aligned}
W\left[p_{\Delta}(\{\boldsymbol{R}\})\right]:= & \left\langle-\frac{k_{\mathrm{B}} T}{\zeta} \sum_{i, j=1}^{N} \frac{\partial p_{\Delta}(\{\boldsymbol{R}\})}{\partial \boldsymbol{R}_{i}} \cdot \boldsymbol{H}_{i, j}\left(\boldsymbol{R}_{i}-\boldsymbol{R}_{j}\right) \frac{\partial p_{\Delta}(\{\boldsymbol{R}\})}{\partial \boldsymbol{R}_{j}}\right. \\
& \left.+2 \sum_{i=1}^{N} \frac{\partial p(\{\boldsymbol{R}\})}{\partial \boldsymbol{R}_{i}} \cdot \dot{\gamma} \boldsymbol{R}_{i}\right\rangle_{\mathrm{eq}}
\end{aligned}
$$

In the limit of vanishing hydrodynamic interactions, $\boldsymbol{H}_{i, j}=\delta_{i, j} \mathbf{1}$, one has

$$
W\left[p_{\Delta}(\{\boldsymbol{R}\})\right]:=\left\langle-\frac{k_{\mathrm{B}} T}{\zeta} \sum_{i=1}^{N} \frac{\partial p_{\Delta}(\{\boldsymbol{R}\})}{\partial \boldsymbol{R}_{i}} \cdot \frac{\partial p_{\Delta}(\{\boldsymbol{R}\})}{\partial \boldsymbol{R}_{i}}+2 \dot{\gamma} \sum_{i=1}^{N} \frac{\partial p_{\Delta}(\{\boldsymbol{R}\})}{\partial \boldsymbol{R}_{i}} \cdot \dot{\boldsymbol{K}} \boldsymbol{R}_{i}\right\rangle_{\mathrm{eq}}
$$


where $\boldsymbol{K}:=\dot{\gamma} / \dot{\gamma}$. A simple choice for the trial function which satisfies $(\overline{\mathrm{I} .2})$ is

$$
p_{\Delta}(\{\boldsymbol{R}\}):=1+\frac{\lambda}{2 a^{2}} \sum_{i, j=1}^{N}\left[\mathbb{1}-E_{0}\right]_{i, j} \boldsymbol{R}_{i} \cdot \boldsymbol{K} \boldsymbol{R}_{j}
$$

Inserting the gradient

$$
\frac{\partial p_{\Delta}(\{\boldsymbol{R}\})}{\partial \boldsymbol{R}_{i}}=\frac{\lambda}{a^{2}} \sum_{j=1}^{N}\left[\mathbb{1}-E_{0}\right]_{i, j} \boldsymbol{K} \boldsymbol{R}_{j}
$$

into (I.4) one ends up with

$$
\eta \geq \frac{k_{\mathrm{B}} T}{\dot{\gamma}^{2} N}\left\langle\left(2 \dot{\gamma} \frac{\lambda}{a^{2}}-\frac{k_{\mathrm{B}} T}{\zeta} \frac{\lambda^{2}}{a^{4}}\right) \sum_{i, j=1}^{N}\left[\mathbb{1}-E_{0}\right]_{i, j} \boldsymbol{R}_{i} \cdot \boldsymbol{K}^{\dagger} \boldsymbol{K} \boldsymbol{R}_{j}\right\rangle_{\mathrm{eq}}
$$

where the projector property $\left(\mathbb{1}-E_{0}\right)^{2}=\mathbb{1}-E_{0}$ was employed. Maximization with respect to $\lambda$ gives $\lambda_{\max }=\dot{\gamma} \zeta a^{2} /\left(k_{\mathrm{B}} T\right)$. Due to rotational invariance of the potential energy one has $\left\langle\boldsymbol{R}_{i} \cdot \boldsymbol{K}^{\dagger} \boldsymbol{K} \boldsymbol{R}_{j}\right\rangle_{\text {eq }}=1 / d\left\langle\boldsymbol{R}_{i} \cdot \boldsymbol{R}_{j}\right\rangle_{\text {eq }}$ and enventually

$$
\eta \geq \frac{\zeta}{d N}\left\langle\sum_{i, j=1}^{N}\left[\mathbb{1}-E_{0}\right]_{i, j} \boldsymbol{R}_{i} \cdot \boldsymbol{R}_{j}\right\rangle_{\mathrm{eq}}
$$

This can be written in the following form

$$
\eta \geq \frac{\zeta}{2 d}\left\langle\sum_{k=1}^{K} \frac{N_{k}}{N} R_{\mathrm{gyr}}^{2}\left(\mathscr{N}_{k}\right)\right\rangle_{\mathrm{eq}}
$$

In the absence of excluded volume interactions, the bound coincides with the exact value in the Rouse model which can be inferred from (4.37) and (5.19). 


\section{Bibliography}

[AdDe85] M. Adam, M. Delsanti, D. Durand

Mechanical measurements in the reaction bath during the polycondensation reaction, near the gelation threshold

Macromolecules 18, 2285 (1985)

[AdDe88] M. Adam, M. Delsanti, J. P. Munch, D. Durand

Dynamical studies of polymeric cluster solutions obtained near the gelation threshold - glasslike behaviour

Phys. Rev. Lett. 61, 706 (1988)

[AdDe79] M. Adam, M. Delsanti, R. OKasha, G. Hild

Viscosity study in the reaction bath of the radical copolymerization of styrene divinybenzene

J. Physique Lett. 40, L539 (1979)

[AdLa96] M. ADAM, D. LAIREZ

Sol gel transition

in:

J. P. COHEN ADDAD

Physical properties of polymeric gels

Wiley, New York (1996)

[AdLa91] M. Adam, D. Lairez, F. Boué, J. P. Busnel, D. Durand, T. Nicolai

Verifi cation of the hyperscaling law on polymer clusters by neutron-scattering experiments

Phys. Rev. Lett. 67, 3456 (1991)

[AdLa97] M. AdAm, D. Lairez, M. Karpasas, M. GotTlieb

Static and dynamic properties of cross-linked Poly(dimethylsiloxane) pregel clusters

Macromolecules 30, 5920 (1997)

[Alb72] A. E. ALBERT

Regression and the Moore-Penrose pseudoinverse

Academic Press, New York (1972)

[Ale84] S. AleXANDER

Is the elastic energy of amorphous materials rotationally invariant?

J. Physique (Paris) 45, 1939 (1984)

[AlOr82] S. Alexander, R. ORbaCh

Density of states of fractals: 'fractons'

J. Physique (Paris) 43, L625 (1982) 
[ArSa90] S. ARbabi, M. SAhimi

Critical properties of viscoelasticity of gels and elastic percolation networks

Phys. Rev. Lett. 65, 725 (1990)

[ArJh81] R. C. Armstrong, M. S. JhON

On the stability criteria of the Kirkwood-Riseman diffusion tensor

J. Chem. Phys. 75, 4160 (1981)

[Arn92] L. ARNOLD

Stochastic differential equations: Theory and applications

Krieger, Malabar (1992)

[AxKo90] M. A. V. Axelos, M. KolB

Crosslinked biopolymers: Experimental evidence for scalar percolation theory

Phys. Rev. Lett. 64, 1457 (1990)

[BaEd80] R. C. BALL, S. F. EdWARdS

Elasticity and stability of a dense gel

Macromol. 13, 748 (1980)

[BaBu92] J. BAUER, W. BURCHARD

Critical behaviour of trifunctional randomly branched polycyanurates

J. Phys. II (France) 2, 1053 (1992)

[BeOr78] C. M. Bender, S. A. OrsZaG

Advanced mathematical methods for scientists and engineers

McGraw-Hill, New York (1978)

[BePe76] B. J. Berne, R. PeCorA

Dynamic light scattering

Wiley, New York (1976)

[BiCu87] R. B. Bird, C. F. Curtiss, R. C. Armstrong, O. Hassager

Dynamics of Polymeric Liquids, Vols. 1,2

Wiley, New York (1987)

[BIZi66] V. BLOOMFIELD, B. H. ZIMM

Viscosity, sedimentation, et cetera, of ring-and straight-chain polymers in dilute solution

J. Chem. Phys. 44, 315 (1966)

[Bol85] B. BOLlobÁs

Random graphs

Academic Press, London (1985)

[BoYi91] J. P. Boon, S. YIP

Molecular hydrodynamics

Dover, New York (1991)

[BrRo88] A. J. BRAY, G. J. Rodgers

Diffusion in a sparsely connected space: A model for glassy relaxation

Phys. Rev. B 38, 11461 (1988)

[BrAs01] K. Broderix, T. Aspelmeier, A. K. Hartmann, A. Zippelius

Stress relaxation of near-critical gels

Phys. Rev. E 64, 021404 (2001) 
[BrGo97] K. Broderix, P. M. Goldbart, A. Zippelius

Dynamical signatures of the gelation transition

Phys. Rev. Lett. 79, 3688 (1997)

[BrLo99] K. Broderix, H. Löwe, P. MÜller, A. ZipPelius

Shear viscosity of a crosslinked polymer melt

Europhys. Lett. 48, 421 (1999)

[BrLo01a] K. Broderix, H. LÖWE, P. MÜller, A. ZipPelius

Critical dynamics of gelation

Phys. Rev. E 63, 011510 (2001)

[BrLo01b] K. BroderiX, H. LÖWE, P. MÜller, A. ZipPelius

Anomalous stress relaxation in random macromolecular networks

Physica A 302, 379 (2001)

[Bro93] W. BROWN (Ed.)

Dynamic light scattering

Clarendon Press, Oxford (1993)

[BuHa96] A. Bunde, S. HAVLIN

in:

A. Bunde, S. HaVlin (Eds.)

Fractals and disordered systems

Springer, Berlin (1996)

[BuSc80] W. Burchard, M. Schmidt, W. H. Stockmayer

Infuence of hydrodynamic preaveraging on quasi-elastic scattering from flexible linear and star-branched macromolecules

Macromolecules 13, 580 (1980)

[CaGo00] H. M. Castillo, P. M. GoldbarT

Semimicroscopic theory of elasticity near the vulcanization transition

Phys. Rev. E 62, 8159 (2000)

[CaGo94] H. M. Castillo, P. M. Goldbart, A. Zippelius

Distribution of localization lengths in randomly crosslinked macromolecular networks

Europhys. Lett. 28, 519 (1994)

[Cat84] M. E. CATES

Statics and dynamics of polymeric fractals

Phys. Rev. Lett. 53, 926 (1984)

[Cat85] M. E. CATES

Brownian dynamics of self-similar macromolecules

J. Physique (France) 46, 1059 (1985)

[Cay89] A. CAYley

A theorem on trees

Quart. J. Math. 23, 376 (1889)

[CiGr03] S. Ciliberti, T. S. Grigera, V. Martin-Mayor, G. Parisi, P. Verrocchio Brillouin and boson peaks in glasses from vector Euclidean random matrix theory

J. Chem. Phys. 119, 8577 (2003) 
[ChWi85] F. Chambon, H. H. WinteR

Stopping of crosslinking reaction in a PDMS polymer at the gel point

Polym. Bull. 13, 499 (1985)

[CoGi93] R. H. Colby, J. R. Gillmor, M. Rubinstein

Dynamics of near-critical polymer gels

Phys. Rev. E 48, 3712 (1993)

[DeEd76] R. T. DEAM, S. F. EdWARdS

The theory of rubber elasticity

Phil. Trans. R. Soc. London A 280, 317 (1976)

[DeBo93] F. Devreux, J. P. Boilot, F. Chaput, L. Malier, M. A. V. Axelos

Crossover from scalar to vectorial percolation in silica gelation

Phys. Rev. E 47, 2689 (1993)

[DoEd88] M. DoI, S. F. EDWARdS

The theory of polymer dynamics

Clarendon Press, Oxford (1988)

[DuDe87] D. Durand, M. Delsanti, M. Adam, J. M. Luck

Frequency dependence of viscoelastic properties of branched polymers near gelation threshold Europhys. Lett. 3, 297 (1987)

[Eic72] B. E. EICHINGER Elasticity theory. I Distribution functions for perfect phantom networks

Macromolecules 5, 496 (1972)

[ErMa53] A. Erdélyi, W. Magnus, F. Oberhettinger, F. G. Tricomi

Higher transcendental functions

McGraw-Hill, New York (1953)

[ErRe60] P. ERDŐs, A. RÉNYI

Magyar Tud. Akad. Mat. Kut. Int. Kőzl 5, 17 (1960); reprinted in:

J. SPENCER (Ed.)

P. Erdôs: The art of counting

MIT Press, Cambridge, MA (1973), Chap. 14, Article 324.

[Fel66] W. FELLER

An introduction to probability theory and its applications 2

John Wiley \& Sons, New York (1966)

[Fer61] J. D. FERRY

Viscoelastic properties of polymers

Wiley, New York (1961)

[Fix81] M. FIXMAN

Inclusion of hydrodynamic interaction in polymer dynamical simulations

Macromolecules 14, 1710 (1981)

[Fix83a] M. FIXMAN

Effects of fluctuating hydrodynamic interaction

J. Chem. Phys. 78, 1588 (1983) 
[Fix83b] M. FIXMAN

Effects of fuctuating hydrodynamic interaction

J. Chem. Phys. 78, 1594 (1983)

[Flo41a] P. J. FLORY

Molecular size distribution in three dimensional polymers I. Gelation

J. Am. Chem. Soc. 63, 3083 (1941)

[Flo41b] P. J. FLORY

Molecular size distribution in three dimensional polymers II. Trifunctional branching units

J. Am. Chem. Soc. 63, 3091 (1941)

[Flo41c] P. J. FLORY

Molecular size distribution in three dimensional polymers III. Tetrafunctional branching units

J. Am. Chem. Soc. 63, 3096 (1941)

[Flo92] P. J. FLORY

Principles of polymer chemistry

Cornell University Press, Ithaca (1992)

15th printing

[For76] W. C. FORSMAN

Graph theory and the statistics and dynamics of polymer chains

J. Chem. Phys. 65, 4111 (1976)

[GaAr00] E. Del Gado, L. De Arcangelis, A. Coniglio

Viscosity critical behaviour at the gel point in a 3d lattice model

Eur. Phys. J. E 2, 359 (2000)

[GaAr02] E. Del Gado, L. De ArCangelis, A. Coniglio

Elastic critical behavior in a three-dimensional model for polymer gels

Phys. Rev. E 65, 041803 (2003)

[GiLo90] D. B. GINGOLD, D. J. LobB

Percolative conduction in three dimensions

Phys. Rev. B 42, 8220 (1990)

[Gen75] P.-G. DE GENNES

Critical dimensionality for a special percolation problem

J. Physique (Paris) 36, 1049 (1975)

[Gen76a] P.-G. DE GENNES

On a relation between percolation theory and the elasticity of gels

J. Physique (Paris) 37, L1-L2 (1976)

[Gen76b] P.-G. DE GENNES

Unifying concept of percolation

Recherche 7, 919 (1976)

[Gen77] P.-G. DE GENNES

Critical behaviour for vulcanization processes

J. Physique (Paris) Lett. 38, L-355 (1977) 
[Gen78] P.-G. DE GENNES

Viscosité près d'une transition sol-gel

Comptes Rendus Acad. Sci. (Paris) 286B, 131 (1978)

[Gen79a] P.-G. DE GENNES

Scaling concepts in polymer physics

Cornell University Press, Ithaca (1979)

[Gen79b] P.-G. DE GENNES

Incoherent scattering near a sol-gel transiton

J. Physique (Paris) Lett. 40, L-197 (1979)

[GoRo01] C. Godsil, G. Royle

Algebraic graph theory

Springer, New York (2001)

[GoCa96] P. M. Goldbart, H. Castillo, And A. Zippelius

Randomly crosslinked macromolecular systems: vulcanization transition to and properties of the amorphous solid state

Adv. in Phys. 45, 393 (1996)

[GoGo87] N. Goldenfeld, P. M. GoldbarT

Rigidity and ergodicity for randomly cross-linked macromolecules

Phys. Rev. Lett. 58, 2676 (1987)

[GoGo89a] N. GoldEnFELD, P. M. GoldBART

Microscopic theory for cross-linked macromolecules. I. Broken symmetry, rigidity, and topology

Phys. Rev. A 39, 1402 (1989)

[GoGo89b] N. GoldENFELD, P. M. GoldBART

Microscopic theory for cross-linked macromolecules. II. Replica theory of the transition to the solid state

Phys. Rev. A 39, 1412 (1989)

[GoGo92] N. Goldenfeld, P. M. GoldBART

Dynamical scaling and spontaneous symmetry breaking at the gel point

Phys. Rev. A 45, R5343 (1992)

[GrRh80] I. S. GRADSHTEYN AND I. M. RHYZIK

Table of integrals, series, and products

Academic Press, New York (1980)

[GrMa03] T. S. Grigera, V. Martin-Mayor, G. Parisi, P. Verrocchio

Phonon interpretation of the boson peak in supercooled liquids

Nature 422, 289 (2003)

[Gri89] G. GRIMMETT

Percolation

Springer, New York (1989)

[Gut78] I. GuTMAN

Graph-theoretical formulation of Forsman's equation

J. Chem. Phys. 68, 2523 (1978) 
[HaLu87] A. B. HARRIS, T. C. LUBENSKY

Randomly diluted $x y$ and resistor networks near the percolation threshold

Phys. Rev. B 35, 6964 (1987)

[HaMe87] A. B. Harris, Y. Meir, A. Aharony

Diffusion on percolating clusters

Phys. Rev. B 36, 8752 (1987)

[HaMe90] A. B. Harris, Y. Meir, A. Aharony

Resistance distributions of the random resistor network near the percolation threshold

Phys. Rev. B 41, 4610 (1990)

[HaBe02] S. Havlin, D. Ben-AvRaham

Diffusion in disordered media

Adv. in Physics 51, 187 (2002)

[HeDe84] H. J. Herrmann, B. Derrida, J. Vannimenus

Superconductivity exponents in two- and three-dimensional percolation

Phys. Rev. B 30, 4080 (1984)

[HoHa77] P. C. HohENBERG, B. I. HALPERIN

Theory of dynamic critical phenomena

Rev. Mod. Phys. 49, 435 (1977)

[IsLu80] J. IsAaCson, T. C. LubENSKY

Flory exponents for generalized polymer problems

J. Phys. Lett. (Paris) 41, L469 (1980)

[JaSt01] H. K. Janssen, O. Stenull

Relevance of percolation theory to the vulcanization transition

Phys. Rev. E 64, 026119 (2001)

[Jes02] S. N. JESPERSEN

Cluster diffusion at the gelation point

Phys. Rev. E 66, 031502 (2002)

[JePl03] S. N. JesPersen, M. PlischKe

Transport properties of incipient gels

Phys. Rev. E 68, 021403 (2003)

[KaWe84] Y. Kantor, I. Webman

Elastic properties of random percolating systems

Phys. Rev. Lett. 52, 1891 (1984)

[KIRa93] D. J. KLEIN, M. RANDIĆ

Resistance distance

J. Math. Chemistry 12, 81 (1993)

[Ker83] J. KERTÉSZ

Speculation on a scaling law for superconductor-resistor mixture exponent $s$ in a percolation system J. Phys. A 16, L471 (1983)

[Kir54] J. G. KIRKWOOD

The general theory of irreversible processes in solutions of macromolecules

J. Polym. Sci. 12, 1 (1954) 
[KiRi48] J. G. KIRKWOOD, J. RISEMAN

The intrinsic viscosities and diffusion constants of flexible macromolecules in solution

J. Chem. Phys. 16, 565 (1948)

[KiMu04] W. KIRSCH, P. MÜLLER

Spectral properties of the Laplacian on bond percolation graphs

Preprint, math-ph/0407047 (2004)

[Kue01] M. KÜNTZEL

Kritisches Verhalten in dynamischen Modellen für die Vulkanisation

Diplomarbeit, Universität Göttingen (2001)

[KuLo03] M. KÜNTZel, H. Löwe, P. Müller, A. Zippelius

Diffusion of gelation clusters in the Zimm model

Eur. Phys. J. E 12, 325 (2003)

[LaLi59] L. D. LANDAU, E. M. LifSHits

Fluid mechanics

Pergamon Press, London (1959)

[Lar88] R. G. LARSON

Constitutive equations for polymer melts and solutions

Butterworths, Boston (1988)

[Lea76] P. L. LEATH

Cluster size and boundary distribution near percolation threshold

Phys. Rev. B 14, 5046 (1976)

[Loe99] H. LÖWE

Dynamische Eigenschaften von Polymernetzwerken: Ein graphentheoretischer Zugang

Diplomarbeit, Universität Göttingen (1999)

[LoMu04] H. Löwe, P. Müller, A. ZipPelius

Rheology of gelling polymers in the Zimm model

Preprint, cond-mat/0408182 (2004), accepted for publication in J. Chem. Phys.

[LuWa85] T. C. LUBENSKY, J. WANG

Percolation conductivity exponent t to second order in $\varepsilon=6-d$

Phys. Rev. B 33, 4998 (1985)

[LuMo95] C. P. Lusignan, T. H. Mourey, J. C. Wilson, R. H. Colby Viscoelasticity of randomly branched polymers in the critical percolation class

Phys. Rev. E 52, 6271 (1995)

[LuMo99] C. P. Lusignan, T. H. Mourey, J. C. Wilson, R. H. Colby

Viscoelasticity of randomly branched polymers in the vulcanization class

Phys. Rev. E 60, 5657 (1999)

[Mar88] A. MARITAN

About diffusion processes in disordered systems

J. Phys. A 21, 859 (1988) 
[MaOh86] T. Masuda, Y. Ohta, S. OnOGi

Rheological Properties of Randomly Branched Polystyrenes with Different Molecular Weights between Branch Points

Macromolecules 19, 2524 (1986)

[MaAd88] J. E. Martin, D. Adolf, J. Wilcoxon

Viscoelasticity of near-critical gels

Phys. Rev. Lett. 61, 2620 (1988)

[MaAd89] J. E. Martin, D. Adolf, J. Wilcoxon

Viscoelasticity near the sol-gel transition

Phys. Rev. A 39, 1325 (1989)

[MaAd91] J. E. MARTIN, D. AdolF

The sol-gel transition in chemical gels

Ann. Rev. Phys. Chem. 42, 311 (1991)

[MaWi88] J. E. Martin, J. Wilcoxon

Critical dynamics of the sol-gel transition

Phys. Rev. Lett. 61, 373 (1988)

[MaWi91] J. E. Martin, J. Wilcoxon, J. Odinek

Decay of density fluctuations in gels

Phys. Rev. A 43, 858 (1991)

[MeNa99] K. MEHLHORN, S. NÄHER

LEDA - A platform for combinatorial and geometrical computing

Cambridge University Press, Cambridge (1999)

[MeMo70] A. MeIR, J. W. Moon

The distance between points in random trees

J. Combinatorial Theory 8, 99 (1970)

[Mer89] R. MERRIS

An edge version of the matrix-tree theorem and the Wiener index

Linear Multilinear A. 25, 291 (1989)

[MuGo04] S. Mukhopadhyay, P. M. Goldbart, A. Zippelius

Goldstone fuctuations in the amorphous solid state

Europhys. Lett. 67, 49 (2004)

[Mue03] P. MÜLLER

Critical behaviour of the Rouse model for gelling polymers

J. Phys. A 36, 10443 (2003)

[Mut85] M. MuthuKumar

Dynamics of polymeric fractals

J. Chem. Phys 83, 3161 (1985)

[NaYa94] T. NaKayama, K. Yakubo, R. L. ORbaCh

Dynamical properties of fractal networks - scaling, numerical simulations, and physical realizations Rev. Mod. Phys. 66, 381 (1994) 
[Nit94] K. NitTA

A topological approach to statistics and dynamics of chain molecules

J. Chem. Phys 101, 4222 (1994)

[OoKo83] Y. Oono, M. Конмото

Renormalization group theory of transprot properties of polymer solutions. I. Dilute solutions

J. Chem. Phys. 78, 520 (1983)

[Oet87a] H. C. ÖTTINGER

Generalized Zimm model for dilute polymer solutions under theta conditions

J. Chem. Phys. 86, 3731 (1987)

[Oet87b] H. C. ÖTTINGER

Translational diffusivity from the Zimm model

J. Chem. Phys. 87, 3156 (1987)

[Oet89] H. C. ÖTTINGER

A comparison between simulations and various approximations for Hookean dumbbells with hydrodynamic interactions

J. Chem. Phys. 90, 474 (1989)

[Oet96a] H. C. ÖTTINGER

Stochastic processes in polymer fuids

Springer, Berlin (1996)

[Oet96b] H. C. ÖTtINGER

Velocity fi eld in nondraining polymer chains

Rheol. Acta 35, 134 (1996)

[OeRa89] H. C. ÖTtINGER, Y. RABIN

Diffusion equation versus coupled Langevin equations approach to hydrodynamics of dilute polymer solutions

J. Rheol. 33, 725-743 (1989)

[OeZy92] H. C. ÖTTINGER, W. ZYLKA

On the relaxation spectra for models of dilute polymer-solutions

J. Rheol. 36, 885 (1992)

[Ose10] C. W. OSEEN

Über die Stokessche Formel und über eine Verwandte Aufgabe in der Hydrodynamik

Ark. Mat. Astr. Fys. 6, No. 29, 1 (1910)

[PaCh96] I. H. PARK, E-J. CHOI

Characterization of branched polyethyleneimine by laser light scattering and viscometry

Polymer 37, 313 (1996).

[PeGo00] W. Peng, P. M. Goldbart

Renormalization-group approach to the vulcanization transition

Phys. Rev. E 61, 3339 (2000)

[PeGo01] W. Peng, P. M. Goldbart, A. J. McKane

Connecting the vulcanization transition to percolation

Phys. Rev. E 64, 031105 (2001) 
[PiSt81] R. Pike, H. E. STANLEY

Order propagation near the percolation transition

J. Phys. A 14, L169 (1981)

[PIVe03] M. PlischKe, D. C. Vernon, B. Joós

Model for gelation with explicit solvent effects: Structure and dynamics

Phys. Rev. E 67, 011401 (2003)

[Pra71] S. PRAGER

Variational bounds on the intrinsic viscosity

J. Phys. Chem. 75, 72 (1971)

[Pru18] H. PRÜFER

Neuer Beweis eines Satzes über Permutationen

Arch. Math. Phys. 27, 742 (1918)

[PuSc86] S. Puri, B. Schaub, Y. Oono

Renormalization-group analysis of weak-flow effects on dilute polymer solutions

Phys. Rev. A 34, 3362 (1986)

[RaKa89] Y. RABIN, K. KAWASAKI

Suppression of excluded-volume exponents in shear flow of dilute polymer solutions

Phys. Rev. Lett. 62, 2281 (1989)

[RoPr69] J. Rotne, S. Prager

Variational treatment of hydrodynamic interaction in polymers

J. Chem. Phys. 50, 4831 (1969)

[Rou53] P. E. Rouse

A theory of the linear viscoelastic properties of dilute solutions of coiling polymers

J. Chem. Phys. 21, 1272 (1953)

[RuCo03] M. Rubinstein, R. H. Colby

Polymer physics

Oxford University Press, Oxford (2003)

[Sah94] M. SAHIMI

Applications of percolation theory

Taylor \& Francis, London (1994)

[SaGo85] M. SAHIMI, J. D. GodDard

Superelastic percolation networks and the viscosity of gels

Phys. Rev. B 32, 1869 (1985)

[ScOt84] P. SchÜMMER, B. OTTEN

A complement to the bead/spring theory of polymer solutions: Inclusion of the relationship between microscopic and macroscopic velocity gradients

in:

B. Mena, A. García-Rejón, C. Randel-Nataile (Eds.)

Advances in rheology, 1, Theory

Universidad Nacional Autónoma de México (1984) 
[ShAd02] P. F. Sheridan, D. B. Adolf, A. V. Lyulin, I. NeElov, ANd G. R. Davies

Computer simulations of hyperbranched polymers: The influence of the Wiener index on the intrinsic viscosity and radius of gyration

J. Chem. Phys. 117, 7802 (2002).

[Sim79] B. Simon

Trace ideals and their applications

Cambridge University Press, Cambridge (1979)

[Sta76] D. STAUFFER

Gelation in concentrated critically branched polymer solutions

J. Chem. Soc. Faraday Trans. II 72, 1354 (1976)

[StAh95] D. Stauffer, A. Aharony

Perkolationstheorie

$\mathrm{VCH}$, Weinheim (1995)

[StCo82] D. Stauffer, A. Coniglio, M. Adam

Gelation and critical phenomena

Adv. in Polym. Sci. 44, 103 (1982)

[StJa01] O. STEnull, H. K. JANSSEN

Relevance of percolation theory to the vulcanization transition

Phys. Rev. E 64, 026119 (2001)

[StJa99] O. STEnull, H. K. JAnssen, K. OeRding

Critical exponents for diluted resistor networks

Phys. Rev. E 59, 4919 (1999)

[Ste77] M. J. STEPHEN

Mean-fi eld theory and critical exponents for a random resistor network

Phys. Rev. B 15, 5674 (1977)

[Sti74] R. B. Stinchсомве

Conductivity and spin wave stiffness in disordered systems-and exactly soluble model

J. Phys. C: Solid State Phys. 7, 179 (1974)

[Sto43] W. H. STOCKMAYER

Theory of molecular size distribution and gel formation in branched-chain polymers

J. Chem. Phys. 11, 45 (1943)

[Sto44] W. H. STOCKMAYER

Theory of molecular size distribution and gel formation in branched polymers II. General cross linking

J. Chem. Phys. 12, 125 (1944)

[Str77] J. P. STRALEY

Critical exponents for the conductivity of random resistor lattices

Phys. Rev. B 15, 5733 (1977)

[SyGa81] M. F. Sykes, D. S. Gaunt, M. Glen

Perimeter polynomials for bond percolation processes

J. Phys. A 14, 287 (1981) 
[TaYo94] M. Takahashi, K. Yokoyama, T. Masuda

Dynamic viscoelasticity and critical exponents in sol-gel transition of an end-linking polymer

J. Chem. Phys. 101, 798 (1994)

[TaUr90] T. Takigawa, K. Urayama, T. Masuda

Critical behaviour of the intrinsic viscosity of poly(vinylalcohol) solutions near the gelation point

J. Chem. Phys. 93, 7310 (1990)

[TaTh87] W. TANG, M. F. THORPE

Mapping between random central-force networks and random resistor networks

Phys. Rev. B 36, 3798 (1987)

[Tet91] P. TETALI

Random walks and the effective resistance of networks

J. Theoretical Probability 4, 101 (1991)

[TiTo04] T. Tixier, P. Tordjeman, G. Cohen-Solal, P. H. Mutin

Structural effects on the viscoelasticity of polydimethylsiloxane networks close to the sol-gel

threshold

J. Rheol. 48, 39 (2004)

[ToFa01] P. Tordjeman, C. Fargette, P. H. Mutin

Viscoelastic properties of a cross-linked polysiloxane near the sol-gel transition

J. Rheol. 45, 995 (2001)

[Vil88] T. A. VILGIS

Flory theory of polymeric fractals - intersection, saturation and condensation

Physica A 153, 341 (1988)

[VeP101] D.C. Vernon, M. PlischKe, B. Joós

Viscoelasticity near the gel point: A molecular dynamics study

Phys. Rev. E 64, 031505 (2001)

[Wan89] S. WANG

Langevin approach to polymers in fow

Phys. Rev. A 40, 2137 (1989)

[WaFr88] S. WANG, K. F. FreED

On the equivalence of the Kirkwood diffusion equation to the coupled polymer solvent Langevin dynamics

J. Phys. A 21, 2453 (1988)

[Wig58] E.P. WIGNER

On the distribution of the roots of certain symmetric matrices

Ann. Math. 67, 325 (1958)

[Wie47] H. WIENER

Correlation of heats of isomerization, and differences in heats of vaporization of isomers, among the paraffi $n$ hydrocarbons

J. Am. Chem. Soc 69, 2636 (1947)

[Wie98a] K. J. WIESE

Dynamics of selfavoiding tethered membranes I. Model A dynamics (Rouse model)

Eur. Phys. J. B 1, 269 (1998) 
[Wie98b] K. J. WIESE

Dynamics of selfavoiding tethered membranes II. Inclusion of hydrodynamic interactions (Zimm model)

Eur. Phys. J. B 1, 273 (1998)

[WiMo97] H. H. WINTER, M. MourS

Rheology of polymers near liquid-solid transitions

Adv. Polym. Sci. 134, 165 (1997)

[XiMu04] X. XIng, S. Mukhopadhyay, P. M. Goldbart

Scaling of entropic shear rigidity

Preprint, cond-mat/0406411 (2004)

[Yam70] H. YAMAKAWA

Transport properties of polymer chains in dilute solution: Hydrodynamic interaction

J. Chem. Phys. 53, 436 (1970)

[Zim56] B. H. ZIMM

Dynamics of polymer molecules in dilute solution: Viscoelasticity, fbw birefringence and dielectric loss

J. Chem. Phys. 24, 269 (1956)

[ZiSt49] B. H. Zimm, W. H. STOCKMAYER

The dimensions of chain molecules containing branches and rings

J. Chem. Phys. 17, 1301 (1949)

[ZiGo97] A. Zippelius, P. M. GoldbarT

Vulcanized matter: A model glass

in:

A. P. Young (Ed.)

Spin glasses and random fi elds

World Scientifi c, Singapore (1997)

[ZhTh96] H. Zheng, Q. ZhANG, K. JIANG, H. ZHANG, J. WANG

Critical behaviour of viscosity for alginate solutions near the gelation threshold by cupric ions

J. Chem. Phys. 105, 7746 (1996)

[ZhLa00] Z. Zhou, P.-Y. LAI, B. Joós

Rigorous solution for the elasticity of diluted Gaussian spring networks

Phys. Rev. E 62, 7490 (2000) 


\section{Acknowledgement}

Zum Abschluss möchte ich mich herzlich bei allen bedanken, die mich in den letzen Jahren bei der Fertigstellung dieser Arbeit unterstützt haben. Zuerst möchte ich mich bei Prof. Annette Zippelius für eine engagierte Betreuung der Arbeit in einer entspannten und motivierenden Arbeitsatmosphäre bedanken. Und ich danke Peter Müller für die interessanten Diskussionen mit ihm und für eine äußerst angenehme Zusammenarbeit in den letzten Jahren. Ausserdem danke ich Timo Aspelmeier für die sorgfältige Durchsicht des Manuskripts. Und ein stilles Danke geht an Kurt.

Ein besonderer Dank gilt Gisela Mesecke und allen anderen Mitarbeiterinnen des Instituts für die vielen kleinen Hilfen, Kuchen, Streichhölzer und vor allem auch für ihr Engagement bei Feiern aller Art. Danke auch an Jürgen Holm für ein beispielhaft gepflegtes Rechnernetz.

Ein großes Dankeschön natürlich auch an die Frühstücks- und Kickerrunde, Darth (der Integralkrieger), Christian, Garrit, Stephan und Axel, alle Ex-Villaner, insbesondere Julia, und alle anderen die ich eventuell vergessen haben könnte. Das finale Dankeschön geht an Anja.

Diese Arbeit wurde teilweise gefördert durch die Deutsche Forschungsgemeinschaft im Rahmen der Sonderforschungsbereiche 345 und 602. 


\section{Curriculum Vitae}

Henning Löwe

Reinholdstraße 11

D-37083 Göttingen

\section{Biographische Daten}

geboren am

Staatsangehörigkeit
07.05.1973 in Gronau (Leine)

Deutsch

\section{Ausbildung}

$05 / 1993$

07 / 1996

$07 / 1999$

$12 / 2004$
Abitur

Gymnasium Alfeld

Vordiplom Physik

Georg-August-Universität Göttingen

Diplom Physik

Georg-August-Universität Göttingen

Promotion Physik

Georg-August-Universität Göttingen

Göttingen, 03.11.2004 


\section{List of publications}

[1] K. Broderix, H. Löwe, P. Müller, and A. Zippelius Shear viscosity of a crosslinked polymer melt

Europhys. Lett. 48, (1999), 421

[2] K. Broderix, H. Löwe, P. Müller, and A. Zippelius Critical dynamics of gelation

Phys. Rev. E 63, (2001), 011510

[3] K. Broderix, H. Löwe, P. Müller, and A. Zippelius Anomalous stress relaxation in random macromolecular networks Physica A 302, (2001), 379

[4] M. Küntzel, H. Löwe, P. Müller, and A. Zippelius Diffusion of gelation clusters in the Zimm model Eur. Phys. J. E 12, (2003), 325

[5] H. Löwe, P. Müller, and A. Zippelius Rheology of gelling polymers in the Zimm model Preprint, cond-mat/0408182, (2004) accepted for publication in J. Chem. Phys. 